Florida International University FIU Digital Commons

FIU Electronic Theses and Dissertations

University Graduate School

6-15-2012

\title{
Developing a High Density Pt/Alumina Hermetic Feedthrough
}

Ali Karbasi

akarb002@fiu.edu

DOI: 10.25148 /etd.FI12072704

Follow this and additional works at: https://digitalcommons.fiu.edu/etd

\section{Recommended Citation}

Karbasi, Ali, "Developing a High Density Pt/Alumina Hermetic Feedthrough" (2012). FIU Electronic Theses and Dissertations. 667. https://digitalcommons.fiu.edu/etd/667

This work is brought to you for free and open access by the University Graduate School at FIU Digital Commons. It has been accepted for inclusion in FIU Electronic Theses and Dissertations by an authorized administrator of FIU Digital Commons. For more information, please contact dcc@fiu.edu. 


\section{FLORIDA INTERNATIONAL UNIVERSITY}

Miami, Florida

\section{DEVELOPING A HIGH DENSITY PT/ALUMINA HERMETIC FEEDTHROUGH}

A dissertation submitted in partial fulfillment of the requirements for the degree of DOCTOR OF PHILOSOPHY

in

\section{MATERIALS SCIENCE AND ENGINEERING}

by

Ali Karbasi 
To: Dean Amir Mirmiran choose the name of dean of your college/school

College of Engineering and Computing choose the name of your college/school

This dissertation, written by Ali Karbasi, and entitled Developing a High Density Pt/Alumina Hermetic Feedthrough, having been approved in respect to style and intellectual content, is referred to you for judgment.

We have read this dissertation and recommend that it be approved.

Arvind Agarwal

Jiuhua Chen

Wenzhi Li

W. Kinzy Jones, Major Professor

Date of Defense: June 15, 2012

The dissertation of Ali Karbasi is approved.

choose the name of dean of your college/school Dean Amir Mirmiran choose the name of your college/school College of Engineering and Computing

Dean Lakshmi N. Reddi

University Graduate School

Florida International University, 2012 


\section{DEDICATION}

I dedicate this dissertation to my wife and my parents, who gave me endless support and love. It would be impossible to complete this research without them. 


\section{ACKNOWLEDGMENTS}

I sincerely thank my advisor, Dr. W. Kinzy Jones, for his extensive support throughout the span of my PhD research - without his continued, enthusiastic and critical guidance, it would not have been possible for me to write this page today. He treated me like a son and encouraged me to perfect what I was doing. Working closely with him for almost three years, I learned a lot about multi-tasking ability and time management. He has devoted long hours of discussions during the course of this research, which helped me immensely to understand the issues thoroughly. Apart from mentoring to sharpen my technical abilities, he was equally careful for my personality development. His constant support has helped me to gain important confidence, which, I think, will be helpful for my future career.

I would like to offer my heartfelt thanks to Dr. Andriy Durygin for his continued and extensive support in different aspects of this project. Dr. Durygin spent a lot of time to design, manufacture and modify a small self-fabricated hydrogen furnace. Without that furnace a lot of aspects of this project would not have been possible. He was always available for my questions and clarifying every query.

I would like to thank Ms.Jian Wang for all of her help in different aspects of feedthrough preparation. She showed me for the first time how I can use each machine in the packaging laboratory. She was in the lab every time that I needed to do anything or I had any questions.

I would like to offer my special thank Ross Hrubiak for his limitless help during high temperature X-ray synchrotron experiments. We worked together for more than 16 hours every day. During that time, however Ross taught me all aspects about the synchrotron. I couldn't imagine synchrotron study without him. 
I would also want to offer my special thanks to Ali Hadjikhani for his help in different aspects of this project. Ali helped me with sample preparation and FIB analysis of the sample. He was also available whenever I needed for discussion. He was always ready to help beyond his limit at any hour of the day.

I would like to thank To Dr. Liu, Neal Ricks and John Barchi - they were supportive to my experiments throughout my research period. Without their help, it would not have been possible for me to finish this project in a timely manner. I have learned from all of them about different characterization techniques and have always found them ready to go beyond their limits to help.

I am also thankful to our department staff members - Maite and - Martha. They always offered a warm welcome and shared their experience in reducing my involvement in many administrative and purchase related duties.

I'm also thankful of Dr. Doug Shire (Cornell University). He helped a lot during this project with advise about different aspects of feedthrough development.

I sincerely thank Dr. Arvind Agarwal, Dr. Jiuhua Chen And Dr. Wenzhi Li for serving on my dissertation committee and for timely advice and support through my $\mathrm{PhD}$ research.

I sincerly thank Dr. Surendra Saxena. I was working with him before I joined Dr Jones's group, and during that period of time he taught me a great deal and showed me many new aspects of material. I'm really grateful to him for everything that he taught me.

I also thank Dr. Vadym Drozd, Debrupa, Sadeq, Puneet, Rozzbeh, Shadab, Santanu. They have extended their hands whenever I needed any help from them during my research.

I also thank Expert System Solutions and Haiku Tech for measuring the densification behavior with optical dilatometry experiments. 
I also thank Florida International University Graduate School, Dissertation Evidence Acquisition Fellowship (DEA) and MIT Grant 800000195.

I'm thankful of CHESS facility in Cornell University. This work is based upon research conducted at the Cornell High Energy Synchrotron Source (CHESS), which is supported by the National Science Foundation and the National Institutes of Health/National Institute of General Medical Sciences under NSF award DMR-0936384.

I am grateful to my parents; they were one of my greatest sources of energy in life, with all of their inspiration and support

Lastly, this section will be incomplete without mentioning the most influential person in my life in last 6 years - Sanaz. She is my wife, best friend and the person who has always been with me, through the ups and downs of life in last 6 years. I learned much from her in different aspects of life. I would not have dared to start a $\mathrm{PhD}$ in a foreign country alone and without her support. Apart from all these, having similar academic background, her discussions have given me a lot in this research. She was always available for her input in all the critical moments of this research.

This dissertation is a small gift to her. 


\section{ABSTRACT OF THE DISSERTATION}

\section{DEVELOPING A HIGH DENSITY PT/ALUMINA HERMETIC FEEDTHROUGH by}

Ali Karbasi

Florida International University, 2012

Miami, Florida

\section{Professor W. Kinzy Jones, Major Professor}

Typically, hermetic feedthroughs for implantable devices, such as pacemakers, use a alumina ceramic insulator brazed to a platinum wire pin. This combination of material has a long history in implantable devices and has been approved by the FDA for implantable hermetic feedthroughs. The growing demand for increased input/output (I/O) hermetic feedthroughs for implantable neural stimulator applications could be addressed by developing a new, cofired platinum/alumina multilayer ceramic technology in a configuration that supports 300 plus I/Os, which is not commercially available.

Seven platinum powders with different particle sizes were used to develop different conductive cofire inks to control the densification mismatch between platinum and alumina. Firing profile (ramp rate, burn- out and holding times) and firing atmosphere and concentrations (hydrogen (wet/dry), air, neutral, vacuum) were also optimized. Platinum and alumina exhibit the alloy formation reaction in a reduced atmosphere. Formation of any compound can increase the bonding of the metal/ceramic interface, resulting in enhanced hermeticity. The feedthrough fabricated in a reduced atmosphere demonstrated significantly superior performance than that of other atmospheres. A composite structure of tungsten/platinum ratios graded thru the via 
structure (pure W, 50/50 W/Pt, 80/20 Pt/W and pure $\mathrm{Pt}$ ) exhibited the best performance in comparison to the performance of other materials used for ink metallization.

Studies on the high temperature reaction of platinum and alumina, previously unreported, showed that, at low temperatures in reduced atmosphere, $\mathrm{Pt}_{3} \mathrm{Al}$ or $\mathrm{Pt}_{8} \mathrm{Al}_{21}$ with a tetragonal structure would be formed. Cubic $\mathrm{Pt}_{3} \mathrm{Al}$ is formed upon heating the sample to temperatures above $1350^{\circ} \mathrm{C}$. This cubic structure is the equilibrium state of Pt-Al alloy at high temperatures. The alumina dissolves into the platinum ink and is redeposited as a surface coating. This was observed on both cofired samples and pure platinum thin films coated on a $99.6 \mathrm{Wt} \%$ alumina and fired at $1550{ }^{\circ} \mathrm{C}$. Different mechanisms are proposed to describe this behavior based on the size of the platinum particle. 


\section{TABLE OF CONTENTS}

CHAPTER

PAGE

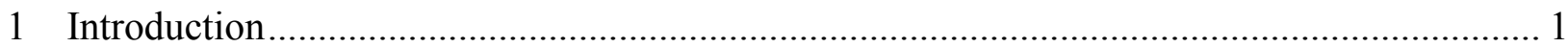

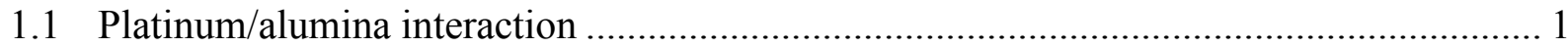

1.2 Design consideration in feedthrough development ....................................................... 2

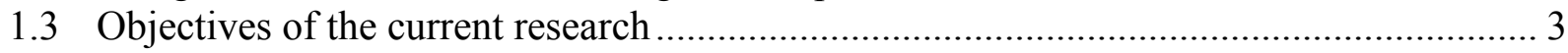

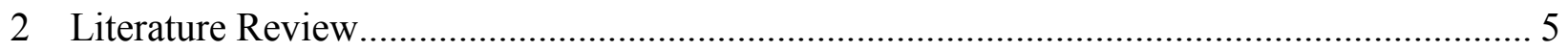

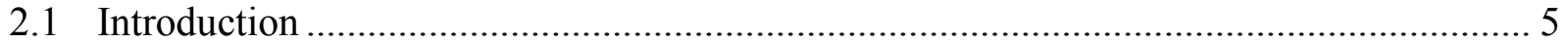

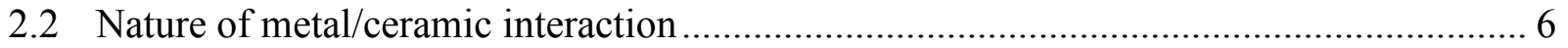

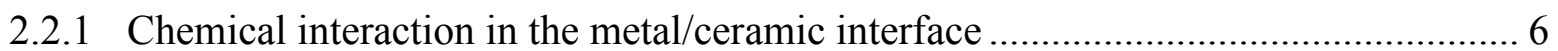

2.2.1.1 Platinum/alumina interaction and bonding ................................................ 9

2.2.2 Physical interaction in the metal/ceramic interface ................................................ 16

2.3 Kinetics and thermodynamics of metal/ceramic interaction ......................................... 19

2.3.1 Kinetics of metal/ceramic interaction................................................................... 19

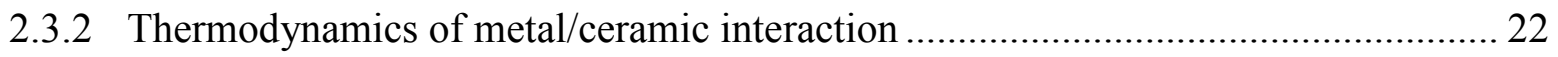

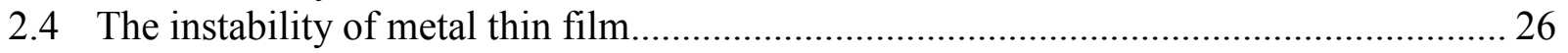

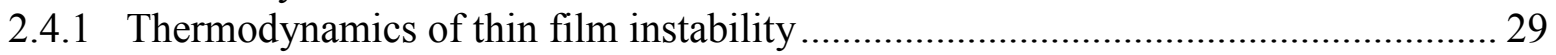

2.4.2 Thermodynamics of thin film sintering and re-dispersion ........................................ 34

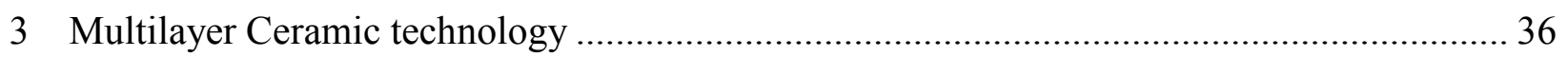

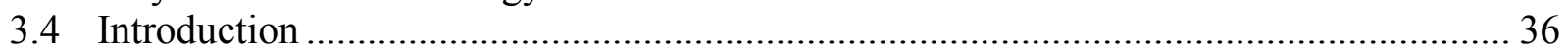

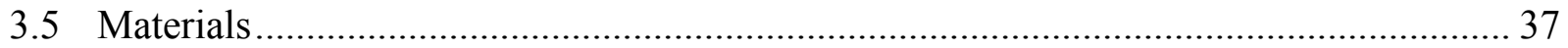

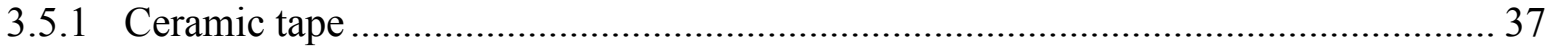

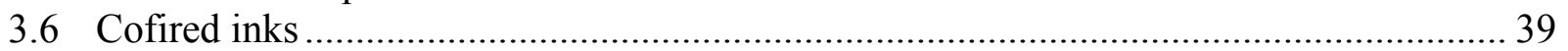

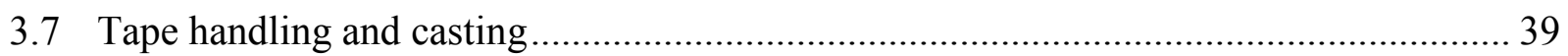

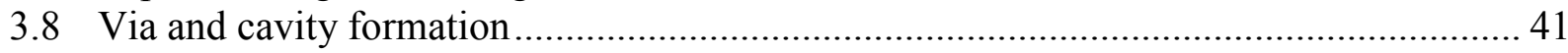

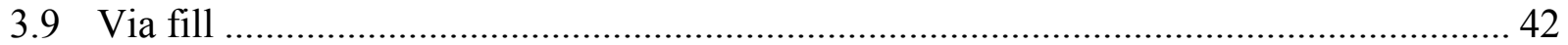

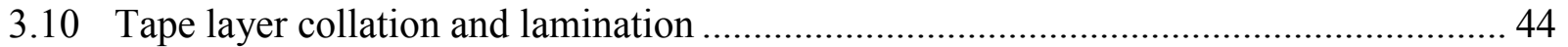

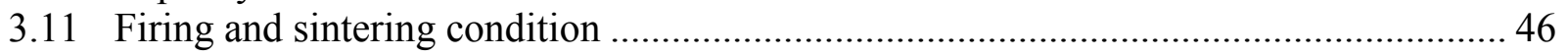

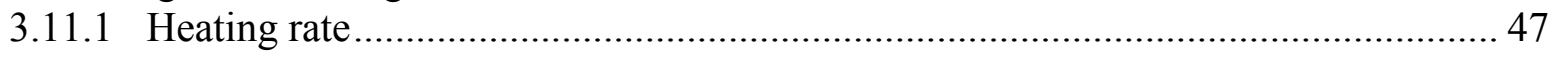

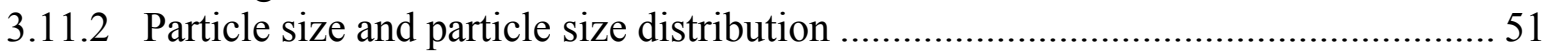

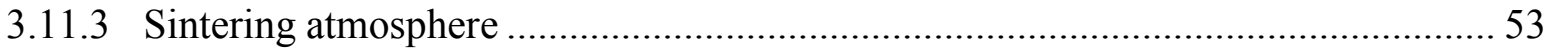

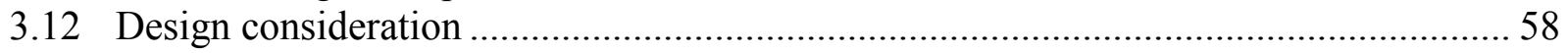

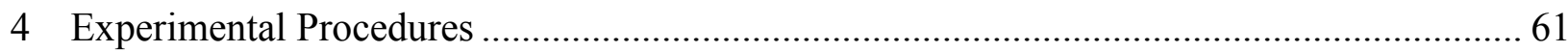

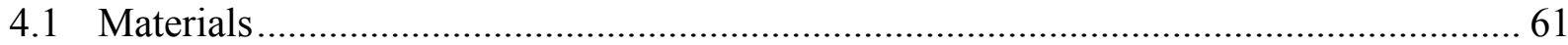

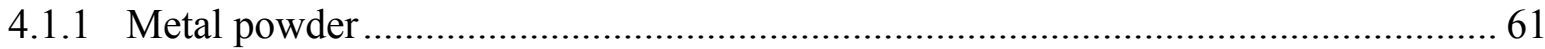

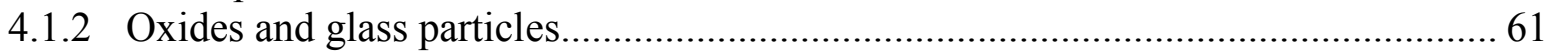

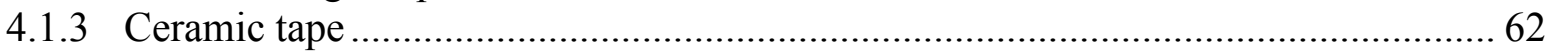

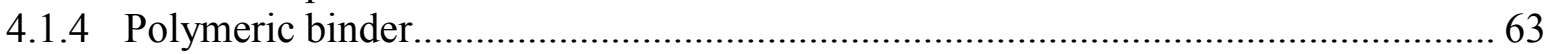

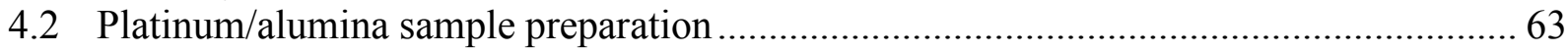

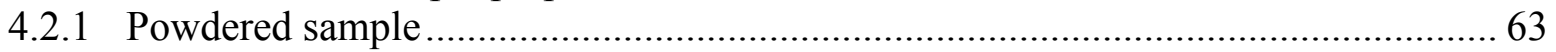




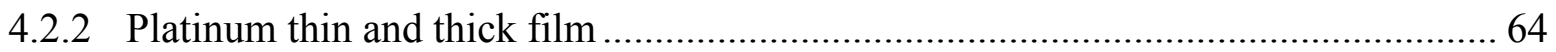

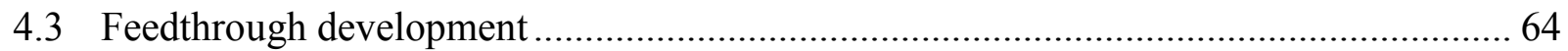

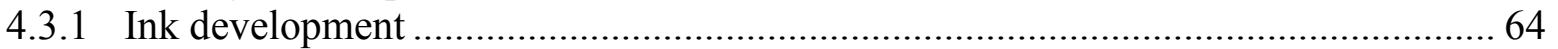

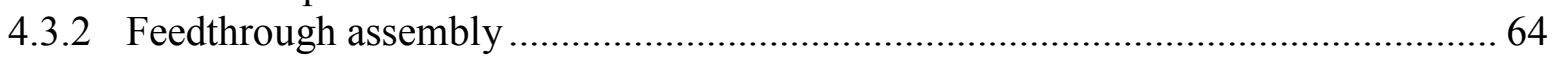

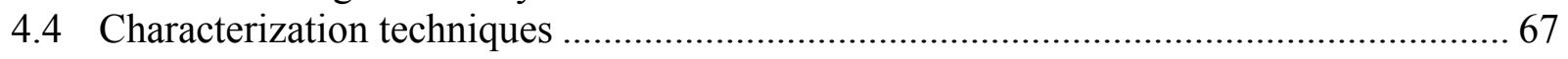

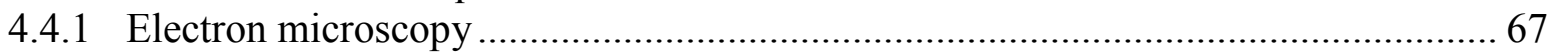

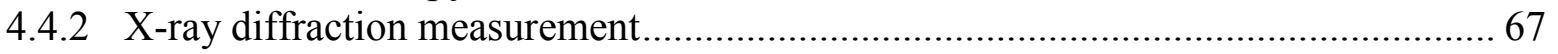

4.4.3 High temperature in-situ X-ray diffraction measurement ............................................... 68

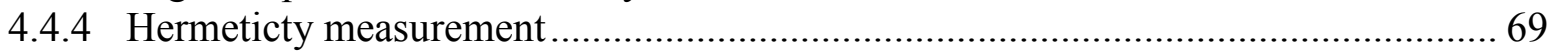

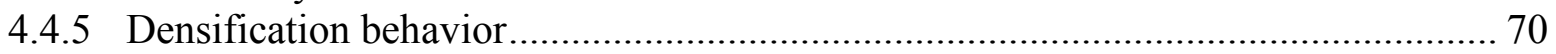

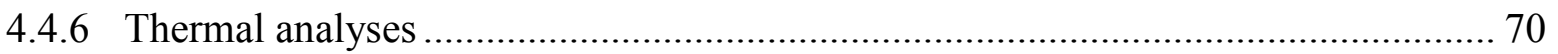

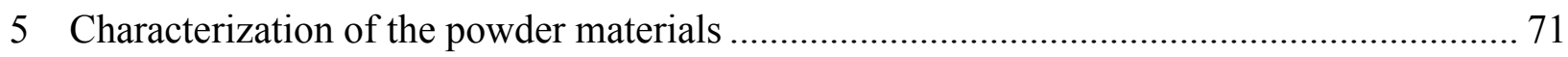

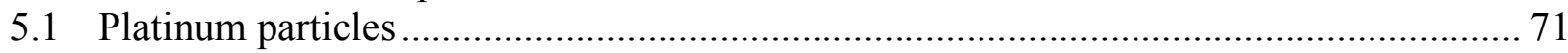

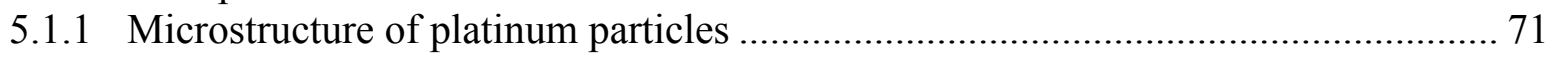

5.1.2 High temperature behavior of platinum particles ..................................................... 73

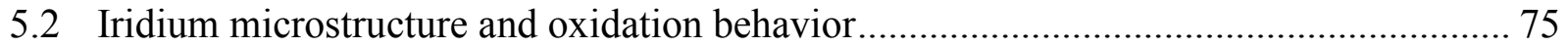

5.3 Sintering and densification behavior of metal particles .................................................. 76

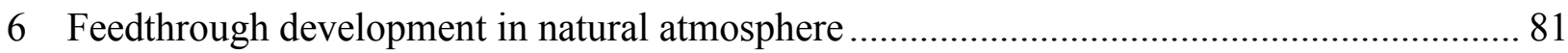

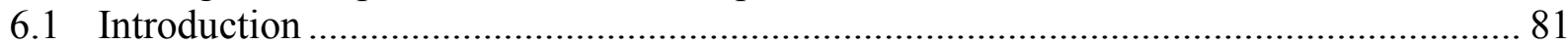

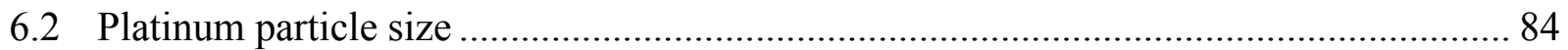

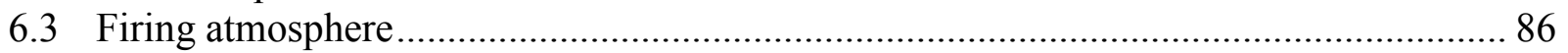

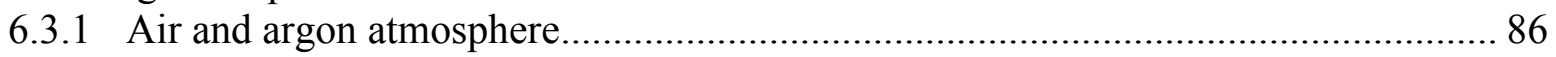

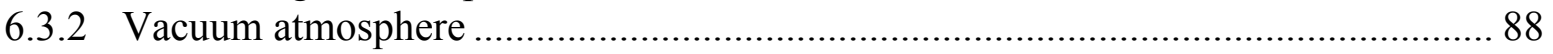

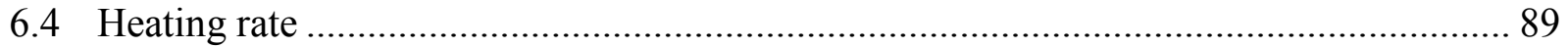

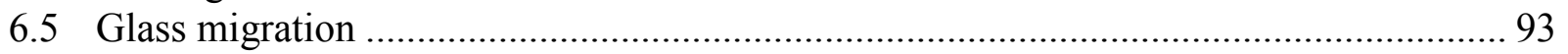

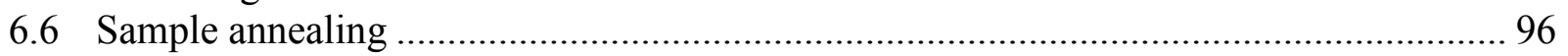

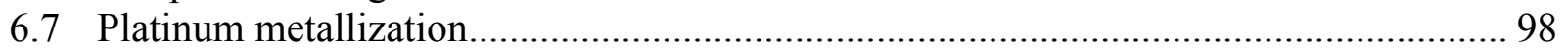

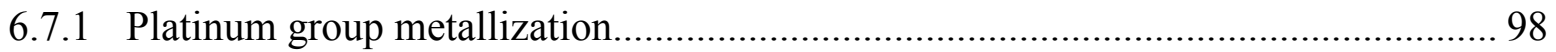

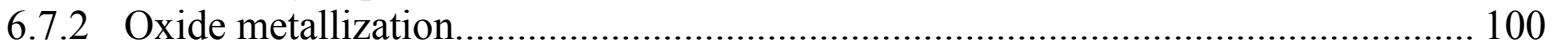

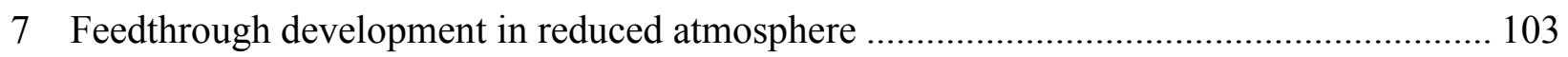

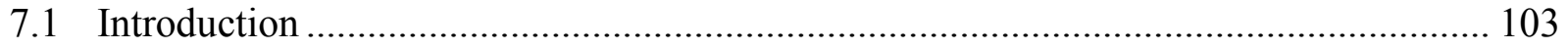

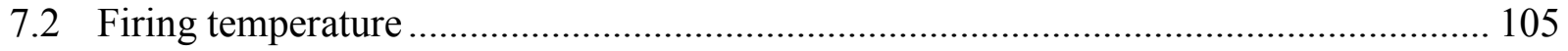

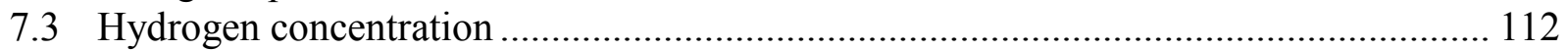

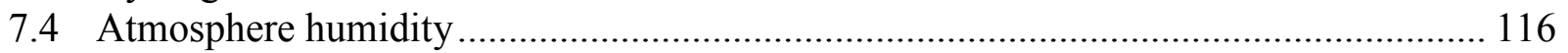

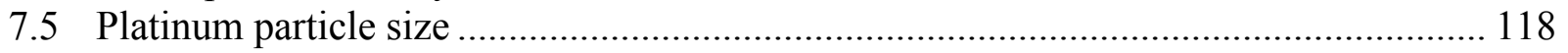

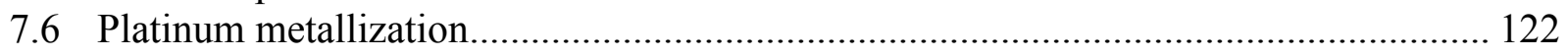

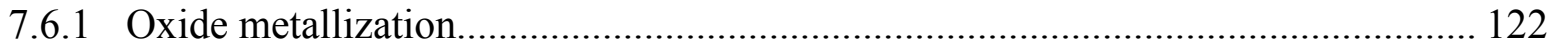

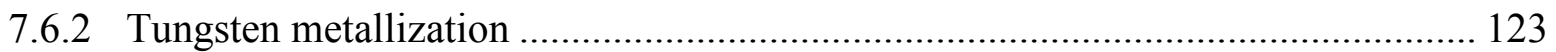

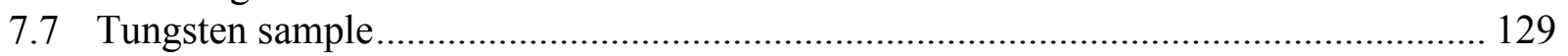

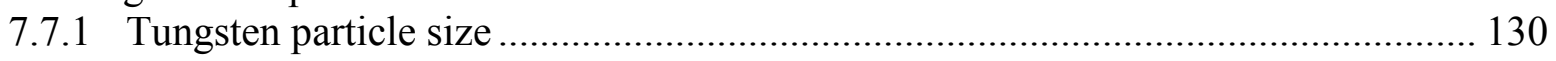

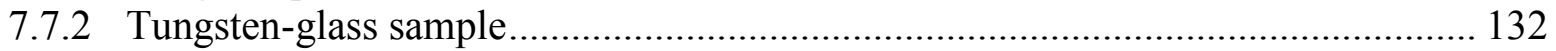

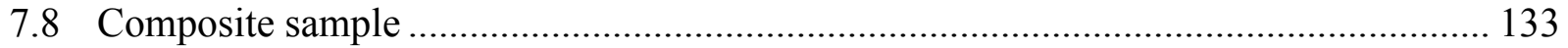




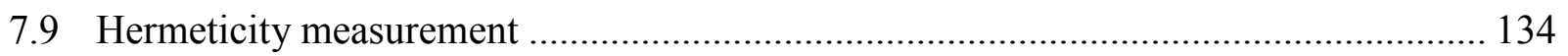

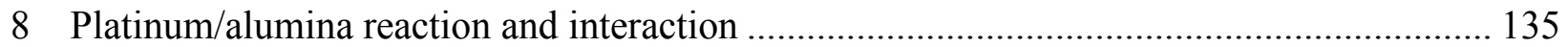

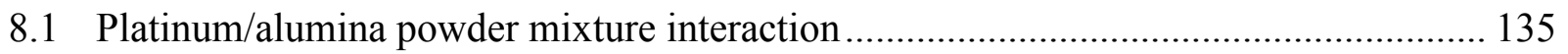

8.1.1 Temperature of the reaction............................................................................ 142

8.2 Platinum thick film interaction with alumina............................................................. 146

8.3 Platinum thin film interaction with alumina ……...................................................... 150

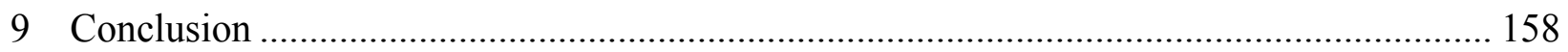

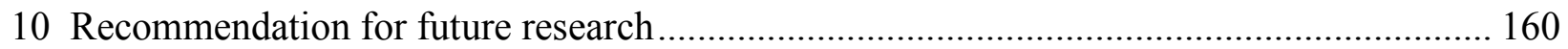

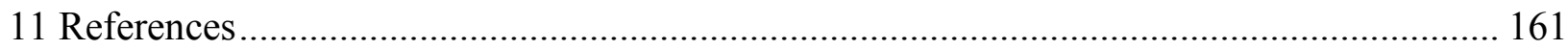

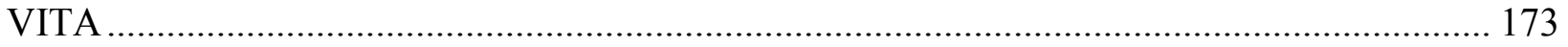




\section{LIST OF FIGURES}

\section{FIGURE}

PAGE

Figure 1-1 Flowchart of the research plan 4

Figure 2-1 The HRTEM image of all four kind of chemical reaction in the surface of metal/oxide (a) Redox reaction of $\mathrm{Nb} / \mathrm{TiO}_{2}$ (110) formed at room temperature. (b) Alloy formation of $5 \% \mathrm{Pt} / \mathrm{CeTbOx}$ catalyst reduced at $1173 \mathrm{~K}$, resulting in the formation of $\mathrm{CePt}_{5}$ alloy. (c) Encapsulation of $\mathrm{Rh}$ by oxide in the $0.5 \% \mathrm{Rh} / \mathrm{Ce}_{0.8} \mathrm{~Tb}_{0.2} \mathrm{O}_{2-\mathrm{X}}$ catalyst reduced at $1173 \mathrm{~K}$. (d) The interface layer of TixCryOz is from the interdiffusion between $\mathrm{Cr}$ overlayers and $\mathrm{TiO}_{2}$ substrate formed at $400{ }^{\circ} \mathrm{C}$.[2]

Figure 2-2 TEM image of Pt/Alumina in reduced atmosphere. (a) the intermetalic layer of $\mathrm{Pt}_{8} \mathrm{Al}_{21}$ formed in $10 \% \mathrm{H}_{2}, 1 \mathrm{hr}, 1073 \mathrm{~K}$ [15]. (b) $\mathrm{Pt}_{3} \mathrm{Al}$ layer fomed in Pure $\mathrm{H}_{2}, 1 \mathrm{hr}, 1073$ $\mathrm{K}[14]$

Figure 2-3 Schematic representation of spillover of a gaseous diatomic from an adsorbing surface onto a non-adsorbing surface, as well as its energy level diagram. 13

Figure 2-4 The transition layer between pt/alumina as observed by Panfilov [4].... 14

Figure 2-5 Platinum precipitates with $c(0001)_{\alpha}\left\|(111)_{P t} ;(10 \overline{10})_{\alpha}\right\|(1 \overline{10})_{P t}$ formed in sapphire after $100 \mathrm{~h}$ at $1600^{\circ} \mathrm{C}$, imaged by (a) bright field-TEM and (b) HRTEM[62]

Figure 2-6 Stages in the distribution of a liquid in a powder bed: (a) pendular stage; (b) funicular

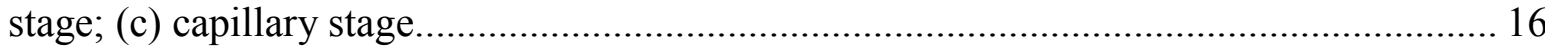

Figure 2-7 Different glass migration flow, (a) Ideal metallizing conditions, (b) Favorable metallizing conditions, (c) Unsatisfactory metallizing conditions (drainage)................... 18

Figure 2-8 The platinum/glass contact angle in different temperature [69] 19

Figure 2-9 Schematic diagrams of kinetics and thermodynamics of encapsulation of metal with $\mathrm{TiO}_{2}$. (a) Energy bands of a metal/TiO 2 interface in the case of $E_{F}($ Metal $)<E_{F}\left(\mathrm{TiO}_{2}\right)$, which creates positive space charges at oxide surface regions to promote the outward diffusion of Ti interstitial ions, $\operatorname{Tin}^{+}(\mathrm{n}<4)$. (b) Mass migration of TiOx $(x<2)$ onto metal clusters driven by the minimization of surface energy of the whole system.

Figure 2-10 Reaction conditions for the $\mathrm{Pt}-\mathrm{Al}_{2} \mathrm{O}_{3}-\mathrm{H}_{2}$ system. Reaction occurs below the line of a

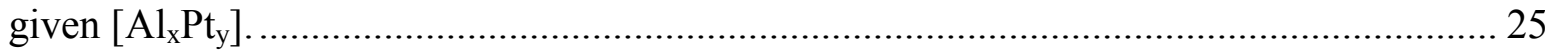


Figure 2-11 Schematic representation of re-dispersion and sintering (a) nonwetting nickel particle in steam before feet formation, (b) the formation of feet on heating in steam, (c) the detachment of the feet from the main body on further heating in steam, (d) the spreading of the feet to form thick films, and (e) formation of a thin surface film

Figure 2-12 A symmetric arrangement of grains in a thin film (a). The grains extend from the free surface down to the substrate. There are two equivalent unit cells in this structure. (b) One grain completely surrounded by grain boundaries. (c) The grain boundary vertex where three grain boundaries meet. (d) A thin film containing an array of holes with radius, which could be used to model the grain boundary vertices.

Figure 2-13 The equilibrium grain boundary groove configuration for a circular cross-section grain. The shape of the free surface is a spherical cap which intersects the grain boundary at an angle $\theta$, the grain diameter is $2 \mathrm{R}$, the film thickness is a, and the equilibrium groove depth is $\mathrm{d}$.

Figure 3-1The process of multilayer ceramic technology .................................................. 37

Figure 3-2 Sintering mechanism of the HTCC tape .............................................................. 40

Figure 3-3Pictures of microvias in $163 \mu \mathrm{m}$ thick LTCC tape. Left) punched via. Right) laserdrilled via [109].

Figure 3-4The isothermal densification behavior and the Arrhenius plot of $\ln (\varepsilon \mathrm{T})$ versus $(1 / T)$.

a) Isothermal sintering. b) non-isothermal sintering

Figure 3-5 Variation of the relative grain size to the relative density of the particles "Sintering path" of alumina particle with different particle size. Experimental data are from Refs. [115, $119,123,124]$

Figure 3-6 Sintering behavior of alumina in different atmosphere. a) Hydrogen, b) Helium, c) Oxygen, d) Nitrogen, f) Argon, g) 7\% Nitrogen+93\% Oxygen

Figure 3-7 The change of catalytic activity of Pt-black catalyst and correspondingly it's densification as a function of time of sintering a $300 \mathrm{C}$ in (1) oxygen atmosphere, (2) nitrogen atmosphere, (3) vacuum and (4) hydrogen atmosphere [129].....

Figure 3-8 Photos of camber development for the $\mathrm{Ag} / \mathrm{LTCC}$ (A) room temperature, (B) $750{ }^{\circ} \mathrm{C}$, (C) $850{ }^{\circ} \mathrm{C}[136]$ 59

Figure 4-1 Punched and filled tape 65

Figure 4-2 Complete feedthrough assembly...... 66 
Figure 4-3 The structure of the small self-fabricated hydrogen furnace 66

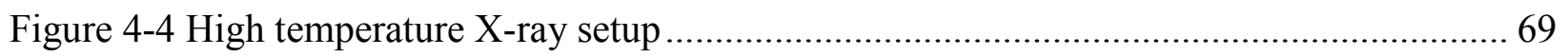

Figure 5-1 SEM image of different platinum particles used in this project................................ 72

Figure 5-2 High magnification images of agglomerated Pt-1 nano particles ......................... 73

Figure 5-3 DCS and TGA results of compressed power .................................................. 74

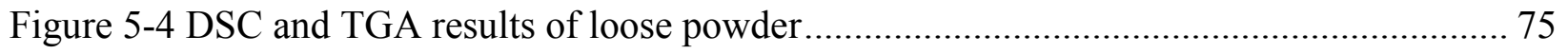

Figure 5-5 SEM image of iridium powder................................................................... 75

Figure 5-6 DSC and TGA results of iridium powder oxidation. (a) TGA curves, (b) DSC curves

Figure 5-7 Densification behavior of different platinum particles in $1550{ }^{\circ} \mathrm{C} \ldots \ldots \ldots \ldots \ldots \ldots \ldots \ldots . . . . . . . . . . . . . .77$

Figure 5-8 Shrinkage rate of Platinum and iridium particles............................................. 78

Figure 5-9 DSC and TGA results of high temperature behavior of platinum, iridium and rhodium

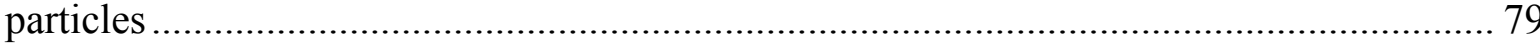

Figure 5-10 Shrinkage behavior of 96\% HTCC tape, nano alumna and Pt-6 platinum powder .. 80

Figure 6-1 Top and bottom side backscatter SEM image of green feedthrough sample............. 81

Figure 6-2 Top and bottom side backscatter SEM image of the ceramic feedthrough after organic

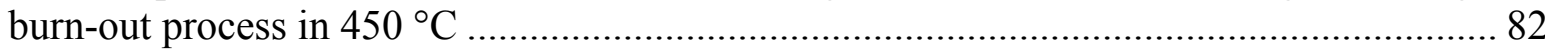

Figure 6-3 Backscatter SEM image of fired a via in ceramic feedthrough ............................. 83

Figure 6-4 Backscatter SEM cross section image of platinum via .......................................... 84

Figure 6-5 SEM image of platinum via with different platinum partcile size. Feedthroughs are

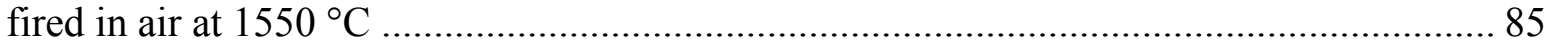

Figure 6-6 SEM image of feedthrough fired in air and argon atmosphere at 1550 C. .............. 86

Figure 6-7 High magnification microscopic image of feedthrough fired in the argon atmosphere

Figure 6-8 DSC and TGA results of firing feedthrough in air and argon atmosphere ............... 88 
Figure 6-9 Backscatter SEM image of feedthrough fired in vacuum 89

Figure 6-10 DSC and TGA results of firing feedthrough with different heating rate in air ......... 90

Figure 6-11 Backscatter SEM image of the platinum via fired in different heating rate. a) $\left.\left.0.2^{\circ} \mathrm{C} / \mathrm{min}, \mathrm{b}\right) 1^{\circ} \mathrm{C} / \mathrm{min}, \mathrm{c}\right) 5^{\circ} \mathrm{C} / \mathrm{min}$ and d) $20^{\circ} \mathrm{C} / \mathrm{min}$ 92

Figure 6-12 SEM image of fired feedthrough in a air atmosphere at $1550{ }^{\circ} \mathrm{C}$............................. 94

Figure 6-13 EDS analyses of the different point in the platinum via from figure 6-13 ............... 95

Figure 6-14 EDS analyses of the cross section of the platinum via fired in the air atmosphere .. 96

Figure 6-15 SEM image of annealed sample for $5 \mathrm{hr}$ at $1550{ }^{\circ} \mathrm{C}$.............................................. 97

Figure 6-16 Electron microspoe image of fired feedthrough with different metalization............. 98

Figure 6-17 SEM image of ceramic feedthrough metalized with alumina ................................. 101

Figure 6-18 SEM image of ceramic feedthrough metalized with ytreium oxide ....................... 102

Figure 6-19 EDS mapping of the of ceramic feedthrough metalized with ytreium oxide.......... 102

Figure 7-1 SEM image of feedthrough fired in two different concentration of hydrogen .......... 103

Figure 7-2 Backscatter SEM image of a sample platinum via fired in $\mathrm{H}_{2}-\mathrm{Ar}(25-75)$ atmosphere

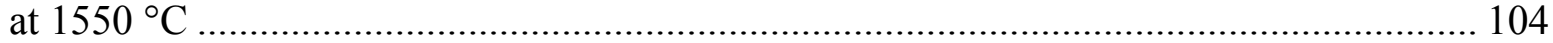

Figure 7-3 EDS elemntal analyses of a sample platinum via fired in $\mathrm{H}_{2}-\mathrm{Ar}(10-90)$ atmosphere at $1550{ }^{\circ} \mathrm{C}$ 105

Figure 7-4 SEM image of two samples via. Feedthrough fired in in $\mathrm{H} 2-\mathrm{Ar}(10-90)$ atmosphere in Camco G-1600 at $1550{ }^{\circ} \mathrm{C}$. 105

Figure 7-5 SEM image of the Pt-4 platinum powder via fired at different temperatures. Feedthroughs are fired in the $\mathrm{H}_{2}-\mathrm{Ar}(25-75)$ atmosphere 106

Figure 7-6 SEM image of the Pt-5 platinum powder via fired at different temperature.

Feedthroughs are fired in the $\mathrm{H}_{2}-\operatorname{Ar}(25-75)$ atmosphere

Figure 7-7 SEM image of Pt-4/alumina boundary area. Feedthrough fired in the $\mathrm{H}_{2}-\operatorname{Ar}(25-75)$ atmosphere at $1550{ }^{\circ} \mathrm{C}$ 
Figure 7-8 Concentration gradient of different element in platinum for the $\mathrm{Pt}-5$ platinum powder.

Feedthrough fired in the $\mathrm{H}_{2}-\mathrm{Ar}(25-75)$ atmosphere at $1550{ }^{\circ} \mathrm{C}$ 110

Figure 7-9 Concentration gradient of different element in platinum for the Pt-5 platinum powder and $99.9 \%$ HTCC ceramic tape. Feedthrough fired in the $\mathrm{H}_{2}-\mathrm{Ar}$ (25-75) atmosphere at $1550{ }^{\circ} \mathrm{C}$

Figure 7-10 Backscatter image of the platinum via from two different particles. Feedthroughs are fired in the $\mathrm{H}_{2}-\mathrm{Ar}(25-75)$ atmosphere at $1550{ }^{\circ} \mathrm{C}$. 112

Figure 7-11 SEM image of Pt-4 platinum via fired at different hydrigen concentration in the mixture with argon. Feedthroughs are fired at $1400{ }^{\circ} \mathrm{C}$. High magnification image shown in left corner.

Figure 7-12 High magnification SEM image of platinum crystals formed in different hydrogen concentration at $1400{ }^{\circ} \mathrm{C}$. 116

Figure 7-13 Back scatter SEM image of Pt-4 feedthough sample fired at $1550^{\circ} \mathrm{C}$ and $10 \% \mathrm{Hr}-$ $90 \% \mathrm{Ar}$ in wet and dry atmosphere.

Figure 7-14 Back scatter SEM image of Pt-5 feedthrough samples fired at $1550^{\circ} \mathrm{C}$ and $10 \% \mathrm{Hr}-$ $90 \% \mathrm{Ar}$ in wet and dry atmosphere.

Figure 7-15 SEM image of platinum via with different platinum partcile size. Feedthroughs are fired in the $\mathrm{H}_{2}-\mathrm{Ar}(10-90)$ atmosphere at $1550{ }^{\circ} \mathrm{C}$. 118

Figure 7-16 SEM image of the Pt-7 platinum powder. Feedthrough fired in the $\mathrm{H}_{2}-\mathrm{Ar}(10-90)$ atmosphere at $1550{ }^{\circ} \mathrm{C}$

Figure 7-17 SEM image of platinum via with the mixture of different platinum particles.

Feedthroughs are fired in the $\mathrm{H}_{2}-\mathrm{Ar}(10-90)$ atmosphere at $1550{ }^{\circ} \mathrm{C}$.

Figure 7-18 SEM image of Pt-7 platinum via. Feedthroughs are fired in the $\mathrm{H}_{2}-\mathrm{Ar}(10-90)$ atmosphere at $1550{ }^{\circ} \mathrm{C}$

Figure 7-19 Concentration gradient of different element in platinum for the Pt-7 $+20 \% \mathrm{Pt}-4$ platinum powder. Feedthrough fired in the $\mathrm{H}_{2}-\mathrm{Ar}(25-75)$ atmosphere at $1550{ }^{\circ} \mathrm{C}$

Figure 7-20 SEM image of platinum metalized with aluminum oxide and tungsten oxide. Samples fired in the $\mathrm{Hr}-\operatorname{Ar}(10-90)$ atmosphere at $1550{ }^{\circ} \mathrm{C}$ 123

Figure 7-21 Microstrcture of the mixture of W-1 and Pt-4 partciles in different ratio . Samples fired in the $\mathrm{H}_{2}-\mathrm{Ar}(10-90)$ atmosphere at $1550{ }^{\circ} \mathrm{C}$.

Figure 7-22 Densification of the mixture of $\mathrm{W}-1$ and $\mathrm{Pt}-4$ partciles in different ratio . Samples fired in the $\mathrm{H}_{2}-\mathrm{Ar}(10-90)$ atmosphere at $1550{ }^{\circ} \mathrm{C}$. 
Figure 7-23 SEM image of tungsten metalized with platinum (W-1 + 20\% Pt-4). Feedthrough fired in the $\mathrm{H}_{2}-\mathrm{Ar}(10-90)$ atmosphere at $1550{ }^{\circ} \mathrm{C}$. High magnification image shown in left corner

Figure 7-24 SEM image of submicron tungsten metalized with platinum (W-2 + 20\% Pt-4). High magnification image in left corner shows the backscatter image. Feedthrough was fired in the $\mathrm{H}_{2}-\mathrm{Ar}(10-90)$ atmosphere at $1550{ }^{\circ} \mathrm{C}$

Figure 7-25 SEM image of submicron tungsten metalized with platinum (W-2 / Pt-4).

Feedthrough was fired in the $\mathrm{H}_{2}-\mathrm{Ar}(10-90)$ atmosphere at $1550{ }^{\circ} \mathrm{C}$

Figure 7-26 SEM image of submicron tungsten metalized with platinum (W-2 / Pt-5).

Feedthrough was fired in the $\mathrm{H}_{2}-\operatorname{Ar}(10-90)$ atmosphere at $1550{ }^{\circ} \mathrm{C}$

Figure 7-27 Backscatter cross-section image of W-2/Pt-5 (50-50) sample. Feedthrough was fired in the $\mathrm{H}_{2}-\mathrm{Ar}(10-90)$ atmosphere at $1550{ }^{\circ} \mathrm{C}$ 128

Figure 7-28 SEM image of feedthrough with micron sized tungsten particle (W-1). High magnification image in left corner shows the backscatter image. Feedthrough was fired in the $\mathrm{H}_{2}-\mathrm{Ar}(10-90)$ atmosphere at $1550{ }^{\circ} \mathrm{C}$

Figure 7-29 SEM image of feedthrough with commerical available tungsten paste from AdTech Cearmic Co. High magnification image in left corner shows the backscatter image. Feedthrough was fired in the $\mathrm{H}_{2}-\mathrm{Ar}(10-90)$ atmosphere at $1550{ }^{\circ} \mathrm{C}$ 130

Figure 7-30 SEM image of feedthrough with submicron sized tungsten particle (W-2). High magnification image in left corner shows the backscatter image Feedthrough was fired in the $\mathrm{H}_{2}-\mathrm{Ar}(10-90)$ atmosphere at $1550^{\circ} \mathrm{C}$.

Figure 7-31 SEM image of feedthrough with 50-50 mixture of two tungsten particles.

Feedthrough is fired in $10 \% \mathrm{H}_{2}-90 \% \mathrm{Ar}$ atmosphere at $1550 \mathrm{c}$

Figure 7-32 SEM image of feedthrough with mixture of micron sized tungsten particles and $20 \%$ glass. Feedthrough was fired in the $\mathrm{H}_{2}-\mathrm{Ar}(10-90)$ atmosphere at $1550{ }^{\circ} \mathrm{C}$. 132

Figure 7-33 Backscatter SEM image of cross-section of the composite sample..... 133

Figure 8-1 Pt-Al Phase diagram. 136

Figure 8-2 Optical image of Pt/Alumina samples fired at the $\mathrm{H}_{2}-\mathrm{Ar}$ (5-95) atmosphere for 8 hour 136

Figure 8-3 Optical image of Pt/Alumina samples fired in air for 8 hours 137 
Figure 8-4 SEM image of spherical platinum particles formed on the surface of Pt65 sample at $1600{ }^{\circ} \mathrm{C}$

Figure 8-5 Densification behavior of the Pt/Alumina samples in two different atmosphere heated at $1400{ }^{\circ} \mathrm{C}$ 138

Figure 8-6 X-ray diffraction results of different platinum/alumina samples fired in hydrogen at

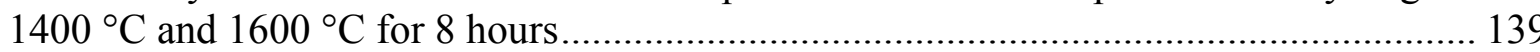

Figure 8-7 Backscatter SEM image of Pt65 sample as-is ......................................................... 140

Figure 8-8 X-ray diffraction results of Pt65 sample fired in hydrogen and air at $1400 \mathrm{C}$ for 8 hours.

Figure 8-9 Backscatter SEM image of Pt65 sample fired in different temperatures and atmospheres.

Figure 8-10 SEM image of Pt65 sample fired in $\mathrm{H}_{2}$ - $\mathrm{Ar}$ (5-95) atmosphere for 8 hours at $1600{ }^{\circ} \mathrm{C}$ 142

Figure 8-11 Microstructure of the alumina coating on the surface of the platinum in the Pt65 sample heated in hydrogen at $1600 \mathrm{C}$ 143

Figure 8-12 X-ray diffraction results of Pt65 sample heated in H2-Ar (25-75) atmosphere at different temperatures

Figure 8-13 Backscatter image of Pt65 sample fired at different temeprature at the H2-Ar (25-75) atmosphere. Samples heated with $10^{\circ} \mathrm{C} / \mathrm{min}$ and kept on that temperature for 1 hour..... 144

Figure 8-14 In situ X-ray diffraction results of Pt65 sample in different temperature 145

Figure 8-15 Backscatter SEM image of thick film of platinum on $99.6 \%$ alumina substrate fired at $1550{ }^{\circ} \mathrm{C}$ in $\mathrm{H}_{2}-\mathrm{Ar}(10-90)$ atmosphere for 3 hour 147

Figure 8-16 Backscatter SEM image of thick film of platinum on $99.6 \%$ alumina substrate fired at $1550{ }^{\circ} \mathrm{C}$ in $\mathrm{H}_{2}-\mathrm{Ar}(10-90)$ atmosphere for 3 hours

Figure 8-17 SEM image of thick film of platinum on $99.6 \%$ alumina substrate fired at $1550{ }^{\circ} \mathrm{C}$ in the H2- $\operatorname{Ar}(10-90)$ atmosphere for 3 hours 148

Figure 8-18 $50 \mathrm{~nm}$ platinum thin film heated in the $\mathrm{H}_{2}-\mathrm{Ar}$ atmosphere for 1 hour. 150

Figure 8-19 $200 \mathrm{~nm}$ platinum thin film rapidly heated in the $\mathrm{H}_{2}-\mathrm{Ar}(10-90)$ atmosphere at 1400 ${ }^{\circ} \mathrm{C}$ 
Figure 8-20 $200 \mathrm{~nm}$ platinum thin film heated in $\mathrm{H} 2-\mathrm{Ar}(10-90)$ atmosphere with $10{ }^{\circ} \mathrm{C} / \mathrm{min}$ rate

Figure 8-21 Microstructure of the $50 \mathrm{~nm}$ thin film heated at $1450{ }^{\circ} \mathrm{C}$ in different hydrogen

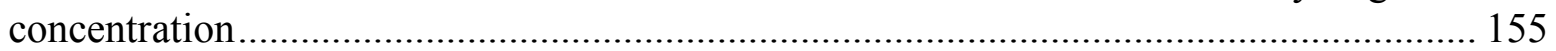

Figure 8-22 Microstructure of the $200 \mathrm{~nm}$ thin film heated at $1450{ }^{\circ} \mathrm{C}$ in different hydrogen

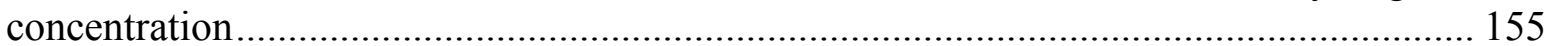

Figure 8-23 Variation of the number density of platinum particles with the particle size for 50 and $200 \mathrm{~nm}$ platinum thin films heated in different temperatures and hydrogen concentration.

Figure 8-24 High magnification of SEM image of platinum crystal from $200 \mathrm{~nm}$ film heated at 1450 in the H2-Ar (20-80) atmosphere. 157 


\section{LIST OF TABLES}

TABLE

PAGE

Table 2-1 Reported reaction between platinum and alumina in literature .............................. 11

Table 2-2 Summary of reactions at solid-solid and solid-gas interfaces ................................ 20

Table 4-1 The properties of the particles used in this project............................................. 62

Table 6-1 Densification behavior of platinum and alumina in different heating rates. .............. 93

Table 6-2 Melting temperature and thermal expansion of different metals and oxide............... 99

Table 7-1 Hermeticty measurements of the different samples ......................................... 134

Table 8-1 Composition of different Pt/Alumina mixture based on different Pt-Al intermetallic

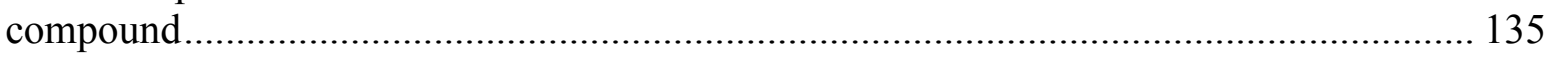

Table 8-2 Boundry condition of the stable-metasatble region for different film thickness........ 151

Table 8-3 Particle size distribution of the $200 \mathrm{~nm}$ platinum thin film heated in different hydrogen

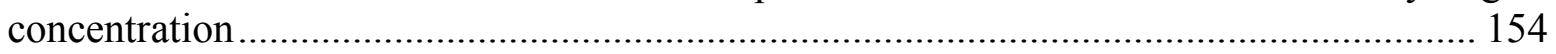

Table 8-4 Particle size distribution of the $50 \mathrm{~nm}$ platinum thin film heated in different hydrogen concentration. 


\section{Introduction}

The feedthrough, a component in the hermetic enclosure, isolates the internal electronic components and hermetically seals them when implanted in the body. A major class of low I/O feedthroughs, such as what can be found in cardiac pacemakers where only 2-4 I/Os are required, are alumina ceramics brazed to a platinum wire pin. This structure has a long and high reliability history in implantable devices, due to biocompatibility of alumina and platinum. The aim of this study is to develop a Pt/Alumina high-density biocompatible feedthrough by utilizing the multilayer ceramic technology, to answer the increasing demand for increased input/output hermetic feedthroughs for application in implantable biomedical devices. Multilayer ceramics technology represents a number of technologies that are capable of producing such a highdensity feedthrough with highly desirable properties.

\subsection{Platinum/alumina interaction}

Strong bonding between metal and ceramic is the most important factor to develop a hermetic device. This bonding can be completely due to physical entanglement of metal and ceramic or it can be chemical in nature. The bonding mechanism in semiconductor type oxide (i.e. $\mathrm{TiO}_{2}, \mathrm{CeO}_{2}$, etc.) and most of the metals, especially the platinum metal group, is chemical in nature [1]. However, in the case of insulator oxide, like $\mathrm{Al}_{2} \mathrm{O}_{3}$ and $\mathrm{SiO}_{2}$, both theoretical and experimental results demonstrate that the electronic interactions between metals and oxide surfaces at low temperatures are dominated by either interfacial bonding or metal polarization effects [2]. Platinum/alumina bonds are particularly interesting because of the proven biocompatibility of both materials [3], yet the true nature of their bonding is not clear[4]. Strong 
bonding between platinum and alumina is believed to be physical in nature [4-6]. However, several studies [1, 7-9] of platinum supported on alumina have reported some changes in the catalytic performance at high temperature application, which is proposed to be caused by the interaction between a metal and an oxide. The support oxide can alter the stability and morphology of the metal particles and the compound formation in the $\mathrm{Pt} / \mathrm{Al}_{2} \mathrm{O}_{3}$ system at high temperatures and probably change the interaction range. Several studies reported the formation

of the intermetallic compound in the interface of $\mathrm{Pt} / \mathrm{Al}_{2} \mathrm{O}_{3}$ in different atmospheres [10-16], but there is no systematic study on platinum/alumina reaction, especially at high temperatures. Yet, the formation of any compound can increase the bonding of the metal/ceramic interface and hermeticity of the final feedthrough.

\subsection{Design consideration in feedthrough development}

The intrinsic multilayer approach of cofired ceramic offers many advantages to designers, however, some consideration is required to develop a proper product. Shrinkage of the cofired ceramic due to the sintering process is occasionally cited as a problem with this technology. Mismatched sintering kinetics between the metal and the ceramic can lead to significant undesirable defects like delamination, cracks, camber or warpage in the product, which substantially lowers the production yield.

Differences in thermal expansion, densification rate mismatch, or combinations of them between the layers could be assumed to be the stress generator. Thermal expansion mismatch is more likely to produce defects in the layer with a larger thermal expansion coefficient during cooling since this layer will be subject to tensile stress. 
To minimize the possibility of forming the co-firing defects, a more thorough understanding of the process resulting in the above defects is needed before the processing parameters and the material compositions can be optimized. Typical solutions for minimizing camber have often relied upon engineering approaches, such as weighting, varying material compositions, and varying the cofiring temperature-time profiles and atmosphere.

\subsection{Objectives of the current research}

The main objective of this research is to develop a high-density biocompatible feedthrough by utilizing the multilayer ceramic technology. This main objective can be achieved through the following specific objectives:

- Analyzing the high temperature densification and thermal behavior of the metal powders and ceramic tape.

- Understanding the role of firing temperature - time profile on the densification and hermeticty of the feedthrough assembly.

- Understanding the role of firing atmosphere on the densification and bonding mechanism of platinum and alumina.

- Studying the effect of different ink metallization on the hermeticity of the final feedthrough assembly.

- Studying the platinum/alumina interaction at high temperatures to understand the bonding mechanism.

Figure 1-1 presents a summary of the research carried out during this study.

The dissertation has been arranged in different chapters, sections and subsections to present the background of the project, the methods adopted in this study, the analysis of the 
results with scientific interpretation and the future scope of research and improvement. The key points of this research with an overview of the main outcomes are described in chapter 9.

Chapter 10 represents the further scope of this research and recommendation for future work.

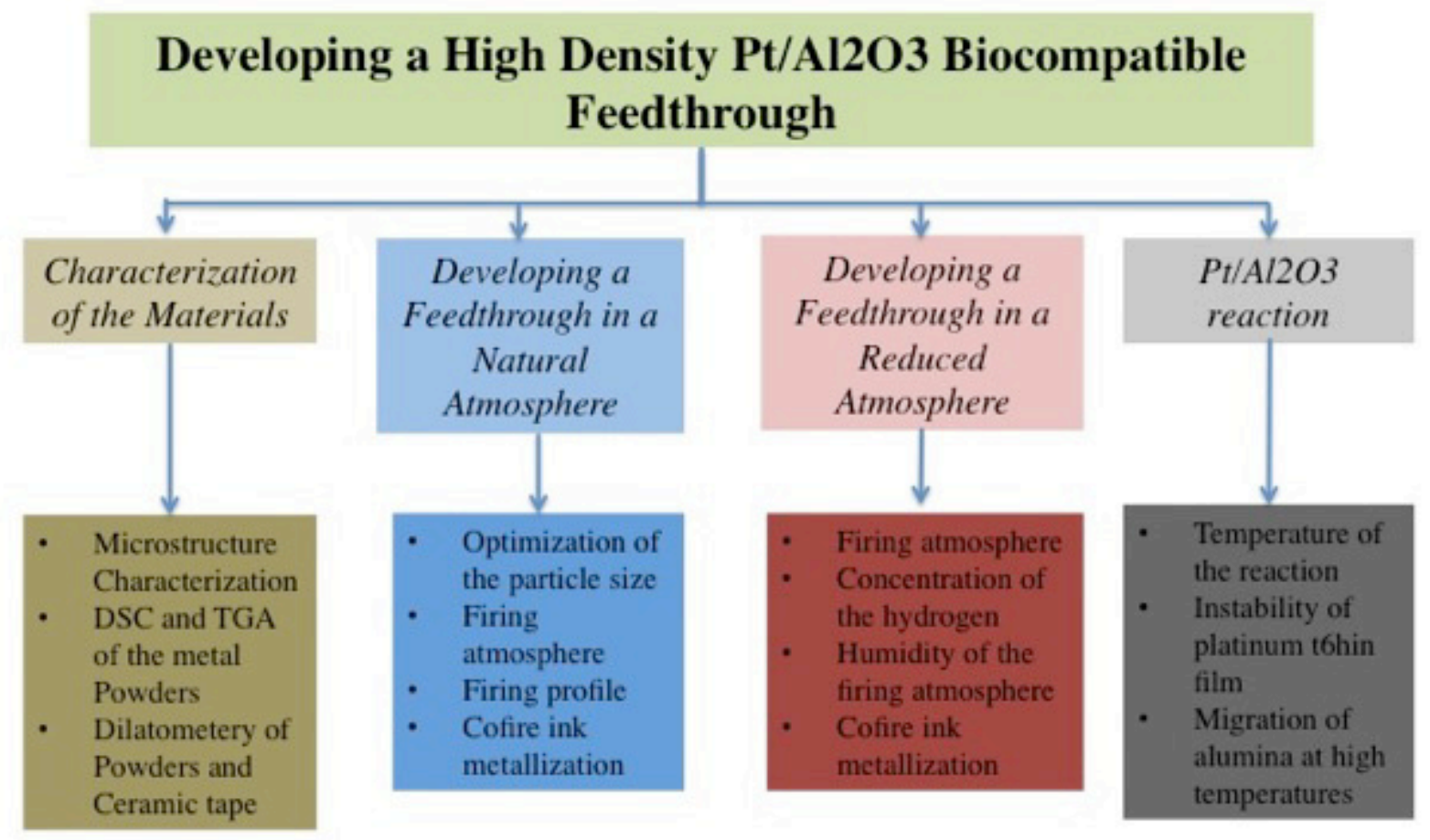

Figure 1-1 Flowchart of the research plan 


\section{Literature Review}

This chapter is presenting a brief review on the metal/ceramic interaction and different bonding mechanism for platinum/alumina joint structure.

\subsection{Introduction}

There is an increasing demand for materials forming hermetic metal/ceramic bonds to use in biomedical engineering, in particular for neurostimulating prosthetic devices such as the cardiac pacemakers, cochlear implants, and vision prosthesis. These bonds isolate the electronic components and hermetically seal them when implanted in the body. There have been many studies that demonstrated strong bonding between ceramics and different types of metals (Examples at ref. $[2,17]$ and references cited in it).

Other than signal feedthrough structure, many technologies and devices, such as functional ceramics with metals, metal matrix composites and heterogeneous catalysts rely upon interfaces between a metal and oxide ceramic. The microstructures, properties, and the lifetimes of multimaterial structures can be affected by the structure, energy, and stability of metal/ceramic interfaces. Currently, the sizes of many materials used for new devices and application are rapidly decreasing to the nanoregime. Gradual miniaturization of devices in microelectronics, catalysis and similar industries leads to an unavoidable increase of the interface-area- to-volume ratio. By increasing the interface, the effect of metal/ceramic interactions becomes more and more significant. Despite their profound impact on materials and device properties, many fundamental properties of ceramic-metal interfaces are not well understood.

From an academic standpoint, a metal/ceramic interface is a contact between two classes of materials that usually differ extremely in the properties due to their different bonding 
characteristics. This difference in material properties could induce stress singularities at the interface and together with the thermal residual stress, can degrade the strength of ceramic/metal joints. This results in deviation in the physical properties of these material combinations. The developed microstructure and interfacial morphology can determine the performance characteristics of the dissimilar material joints $[18,19]$. In the case of interfacial chemical reactions, this can lead to drastic deterioration or significant improvement of the devices performance. For the above reasons, it is of great importance to interfacial the microstructure and the reaction mechanisms of the metal/ceramic interface [20].

Metal films on oxide ceramics have been widely studied in the process of heterogeneous catalysis $[8,21-26]$. The reactivity and stability of such a catalyst depends strongly on the interaction of metal and ceramic. Several studies [1, 8, 9, 27-35] of platinum supported on oxides have reported changes in the catalytic performance in high temperature application due to the sintering and reaction of platinum particles during operation. Such interfacial interactions were found to behave in a complicated manner, encompassing electronic effects (arising from chemical interaction and charge transfer), structural effects (ascribed to structural stabilization), migration of support materials onto the metal, and diffusion and spillover through the metaloxide interface. [16]

\subsection{Nature of metal/ceramic interaction}

\subsubsection{Chemical interaction in the metal/ceramic interface}

It has been found that the chemical interaction between metals and oxide, designated strong metal-support interactions (SMSI), is of great importance in heterogeneous catalysis and significantly affects catalytic properties. Tauster et al. [36] for the first time named the Strong Metal Support Interaction in the $\mathrm{Pt} / \mathrm{TiO}_{2}$ catalysts. Later, the term SMSI was used more generally 
to account for changes in catalytic activity and selectivity in the semiconductor type oxides (i.e. $\mathrm{TiO}_{2}, \mathrm{CeO}_{2}$, etc.) and most of the metals, especially the group VIII elements [1]. Two major factors contribute to the SMSI states: an electronic factor and a geometric factor. The electronic factor is determined by the perturbation of the electronic structure of the metal catalyst, which originates from charge transfer between the metal and the oxide, while the geometric factor results from a thin layer of reduced oxide support physically covering the metal particles (called the encapsulation or decoration model), which blocks active sites at the metal surface. In general, due to the nature of the products formed at metal/oxide interfaces, the chemical interaction can be generally classified into four different groups, as shown in figure 2-1,

- Redox reaction, in which metal is oxidized and supporting oxide is reduced. Of all the reactions occurring at metal/oxide interfaces, these are the most frequently observed, especially for reactive metals on oxides such as $\mathrm{TiO}_{2}$ and $\mathrm{SrTiO}_{3}$. Mass transport in the form of oxygen diffusion (i.e. oxygen vacancy), commonly occurs during the reaction process [37-40].

$$
M e^{I}+M e^{I I} O_{x} \Rightarrow M e^{I} O_{y}+M e^{I I} O_{x-y}
$$

- Alloy formation. At some interfaces, stable intermetallic compounds may be formed. In case of the noble metals like Pt, Pd like Rh, metal on oxide substrate can form the intermetallic compound in hydrogen reduction environment. Pt nanoparticles supported by thin films of silica or alumina in reduced atmosphere systems can form Pt-rich $\mathrm{Pt}_{3} \mathrm{Me}(\mathrm{Me}=\mathrm{Si}, \mathrm{Al})$ alloy phases after the reaction. In cases of reactive metals deposited onto $\mathrm{Al}_{2} \mathrm{O}_{3}$ and $\mathrm{SiO}_{2}$ substrates, the reaction leads to the formation of a thin layer of aluminide or silicide sandwiched between the substrate and a top layer of metal oxide [41-45]. 


$$
M e^{I}+M e^{I I} O_{x} \Rightarrow M e^{I} M e_{y}^{I I}+M e^{I I} O_{z}
$$

Or

$$
M e^{I}+M e^{I I} O_{x} \Rightarrow M e^{I} O_{y}+M e^{I} M e_{z}^{I I}+M e^{I I} O_{x}
$$

- Encapsulation, which is also called decoration, involves mass transport from the oxide support onto the surface of the metal particles. Encapsulation is a special interface process where metal particles are physically covered by a thin layer of reduced oxide support. Noble metals, including $\mathrm{Pt}, \mathrm{Pd}$, and $\mathrm{Rh}$ on $\mathrm{TiO}_{2}$ or $\mathrm{CeO}_{2}$ are good examples of encapsulation reactions $[26,36,46]$.

$$
M e^{I}+M e^{I I} O_{x} \Rightarrow M e^{I I} O_{x-\delta}+M e^{I}+M e^{I I} O_{x}
$$

- Interdiffusion. It is known that metals may diffuse into their oxide supports and/or the substrate atoms may diffuse to the metal surface. Such interdiffusion leads to the formation of interdiffusion zones or mixed oxides (e.g. ternary oxides and oxide solid solutions) at the interfaces. It has been shown that an ion exchange reaction between $\mathrm{Al}$ and spinel ceramic of $(\mathrm{MgO}) \cdot\left(1.25 \mathrm{Al}_{2} \mathrm{O}_{3}\right)$ produces interdiffusion zones at the interface [47-49].

$$
M e^{I}+M e^{I I} O_{x} \Rightarrow M e^{I} M e^{I I} O_{y}
$$

Among all of the mentioned reactions, platinum/alumina reaction is the most important for our study, because their true nature of bonding is not yet known. In the next section different reaction and bonding in platinum/alumina system are reviewed to define the current state of their bonding. 


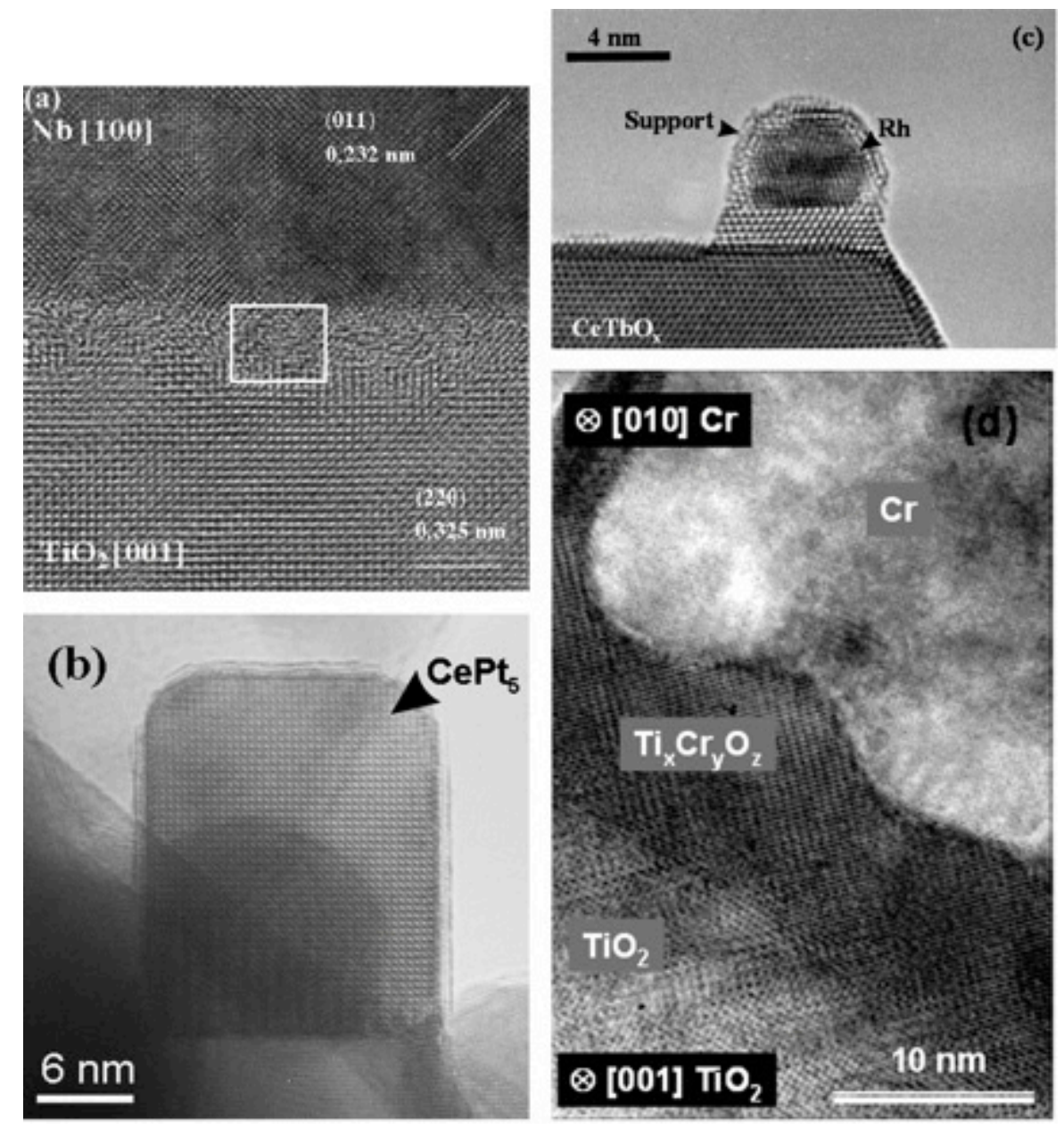

Figure 2-1 The HRTEM image of all four kind of chemical reaction in the surface of metal/oxide (a) Redox reaction of $\mathrm{Nb} / \mathrm{TiO}_{2}(110)$ formed at room temperature. (b) Alloy formation of $5 \% \mathrm{Pt} / \mathrm{CeTbOx}$ catalyst reduced at $1173 \mathrm{~K}$, resulting in the formation of $\mathrm{CePt}_{5}$ alloy. (c) Encapsulation of $\mathrm{Rh}$ by oxide in the $0.5 \%$ $\mathrm{Rh} / \mathrm{Ce}_{0.8} \mathrm{~Tb}_{0.2} \mathrm{O}_{2-} \mathrm{x}$ catalyst reduced at $1173 \mathrm{~K}$. (d) The interface layer of TixCryOz is from the interdiffusion between $\mathrm{Cr}$ overlayers and $\mathrm{TiO}_{2}$ substrate formed at $400{ }^{\circ} \mathrm{C}$.[2]

\subsubsection{Platinum/alumina interaction and bonding}

Schulz et al. [50] first studied the reaction of refractory metals with several oxides in a reduced atmosphere by the $\mathrm{X}$-ray diffraction technique. They showed that the $\mathrm{Pt} / \mathrm{Al}_{2} \mathrm{O}_{3}$ system could go through several alloy formation reactions in temperatures above $1100 \mathrm{C}$. The complete results of the $\mathrm{Pt} / \mathrm{Al}_{2} \mathrm{O}_{3}$ reaction are shown in table 2-1. 
Ruckenstein et al. [51] suggested that the platinum particle splitting in the model catalyst could be due to the stress generation of the formation of the $\mathrm{Pt}_{-} \mathrm{Al}_{2} \mathrm{O}_{3}$ compound. Later [52], TEM diffraction patterns suggested the compound formation between $\mathrm{PtO}_{\mathrm{x}}$ and $\mathrm{Al}_{2} \mathrm{O}_{3}$ yield $\mathrm{Pt}_{\mathrm{p}} \mathrm{Al}_{\mathrm{q}} \mathrm{O}_{\mathrm{r}}$, where $\mathrm{r}$ could be zero. Recently, Luo et al. [16], using photoelectron spectroscopy, also showed the possibility of the formation of the same compound in a vacuum. Analogues to the nickel aluminate (i.e. $\mathrm{NiAl}_{2} \mathrm{O}_{4}$ ), platinum and palladium aluminate could have the same stoichiometric composition [7, 12]. Chu and Ruckenstein [10] also showed that by heating the catalyst in a vacuum at $850^{\circ} \mathrm{C}$ for 6 hours, platinum crystallites can react with the substrate. The TEM electron diffraction identified the sample pattern as a mixed $\mathrm{Pt}_{8} \mathrm{Al}_{21}$, platinum and alumina. Den Otter and Dautzenberg [53], and Kunimori et al. [54] used chemisorption expeimnts to show that $\mathrm{Pt} / \mathrm{Al}_{2} \mathrm{O}_{3}$ can go through alloy formation reaction in the chemisorption process of hydrogn. They concluded that part of the surface alumina in the neighborhood of the platinum crystallites is reduced by the $\mathrm{H}_{2}$ treatment, and reoxidized by the $\mathrm{O}_{2}$ treatment, which leads to a plausible redox reaction. The formula that they proposed only schematically represented and explained the consumed quantity of hydrogen and oxygen gases in the chemisorption experiment.

Chu and Ruckenstein [10] studied the reaction of $\mathrm{Pt} / \mathrm{Al}_{2} \mathrm{O}_{3}$ in the wet hydrogen atmosphere and showed that oxygen oxidizes the platinum to platinum oxide, and hydrogen reduces the oxide generating water between the metal particles and the substrate. This weakens the interaction between metal particles and the substrate and hence enhances crystallite migration. They also showed that the experiment in the wet $\mathrm{N}_{2}$ did not follow the same pattern as wet hydrogen, which could be attributed to the $\mathrm{H}_{2}$ reaction.

It has been shown $[14,55]$ that platinum in the prescence of stable oxide (i.e. $\mathrm{SiO}_{2}$ and $\mathrm{Al}_{2} \mathrm{O}_{3}$ ) in reduced atmopshere can go through alloy formation reaction and create $\mathrm{Pt}_{3} \mathrm{M}(\mathrm{M}: \mathrm{Al}$ 
and $\mathrm{Si}$ ) with the cubic $\mathrm{Cu}_{3} \mathrm{Au}$ (Pm3m space group) structure. Tetragonal $\mathrm{GaPt}_{3}$ crytal structure was also used to anlyse the same stocimetry by Bronger et al. [13]. Zhong et al. [15] showed that the mixtuire of hydrogen/Argon instead of pure hydrogen can change the resultant product. Reaction of $\mathrm{Pt} / \mathrm{Al}_{2} \mathrm{O}_{3}$ in pure hydrogen in $1074{ }^{\circ} \mathrm{K}$ formed the $\mathrm{Pt}_{3} \mathrm{Al}$ compound [14], while the same composition at the same temperature in the $\mathrm{H}_{2}-\mathrm{Ar}(10-90)$ will produce $\mathrm{Pt}_{8} \mathrm{Al}_{21}$ [15].

Table 2-1 Reported reaction between platinum and alumina in literature

\begin{tabular}{|c|c|c|c|c|c|}
\hline Time (hr) & Temperature (C) & Atmosphere & Method & $\mathbf{C o m p o u n d ~}$ & Ref. \\
\hline 12 & 1100 & $\mathrm{H}_{2}$ & X-ray Diffraction & $\mathrm{Pt}_{13} \mathrm{Al}_{3}$ & {$[50]$} \\
\hline 12 & 1200 & $\mathrm{H}_{2}$ & X-ray Diffraction & $\mathrm{Pt}_{3} \mathrm{Al}$ & {$[50]$} \\
\hline 12 & 1250 & $\mathrm{H}_{2}$ & X-ray Diffraction & $\mathrm{Pt}_{5} \mathrm{Al}_{3}$ & {$[50]$} \\
\hline 12 & 1500 & $\mathrm{H}_{2}$ & X-ray Diffraction & $\mathrm{Pt}_{2} \mathrm{Al}_{3}$ & {$[50]$} \\
\hline-- & $>500$ & $\mathrm{O}_{2}$ & Diffraction Pattern (TEM) & $\mathrm{Al}_{2} \mathrm{Pt}_{2} \mathrm{Pt}_{3} \mathrm{O}_{4}$ & {$[52]$} \\
\hline-- & 350 & Vacuum & PES & $\mathrm{Pt}_{\mathrm{x}} \mathrm{Al}_{\mathrm{y}} \mathrm{O}_{\mathrm{z}}$ & {$[16]$} \\
\hline-- & 600 & $\mathrm{O}_{2}$ & TPR & $\mathrm{PtAl}_{2} \mathrm{O}_{4}$ & {$[12]$} \\
\hline 6 & 800 & $V a c u u m$ & Diffraction Pattern (TEM) & $\mathrm{Pt}_{8} \mathrm{Al}_{21}$ & {$[10]$} \\
\hline-- & 500 & $\mathrm{H}_{2}$ & $\mathrm{H}_{2}$ consumption & $\mathrm{Pt}_{2} \mathrm{Al}_{2} \mathrm{O}_{2}$ & {$[54]$} \\
\hline-- & 450 & $\mathrm{PtAl}_{2} .\left(\mathrm{Al}_{2} \mathrm{O}_{3}\right)$ & {$[14]$} \\
\hline $72-168$ & $1200-1300$ & $\mathrm{H}$ & Diffraction Pattern (TEM) & $\mathrm{Pt}_{3} \mathrm{Al}_{2}$ & {$[14]$} \\
\hline 1 & 800 & $\mathrm{H}$ & X-ray Diffraction & $\mathrm{Pt}_{3} \mathrm{Al}_{2}$ & {$[13]$} \\
\hline 1000 & 1500 & $\mathrm{H} 2 / \mathrm{H} 2 \mathrm{O}=105$ & X-ray Diffraction & $\mathrm{Pt}_{8} \mathrm{Al}_{21}$ & {$[15]$} \\
\hline 0.25 & 1500 & $\mathrm{H} 2 / \mathrm{H} 2 \mathrm{O}=5 * 105$ & X-ray Diffraction & $\mathrm{Pt}_{3} \mathrm{Al}_{3}$ & {$[11]$} \\
\hline
\end{tabular}

Hydrogen can react with the oxygen on the interface to create the $\mathrm{Al}^{3+}$ on the interface. Platinum then could migrate over the surface to these freshly formed $\mathrm{Al}^{3+}$ ions, which have been uncovered by the desorption of water and forms a Pt-Al compound [35]. In general, it appears that the hydrogen spillover is the major mechanism for the reaction of $\mathrm{Pt} / \mathrm{Al}_{2} \mathrm{O}_{3}$ in the reduced atmosphere. $\mathrm{H}_{2}$ atoms, migrating from the platinum particle surface to the metal-support interface, induce the reduction of the alumina substrate, accompanied by the interaction with the metal particles to form the interfacial-phase $\mathrm{Pt}_{8} \mathrm{Al}_{21}$. Because of hydrogen spillover to the substrate, the $\mathrm{Pt}-\mathrm{Al}$ alloy is formed not only under platinum particles, but also around them. 


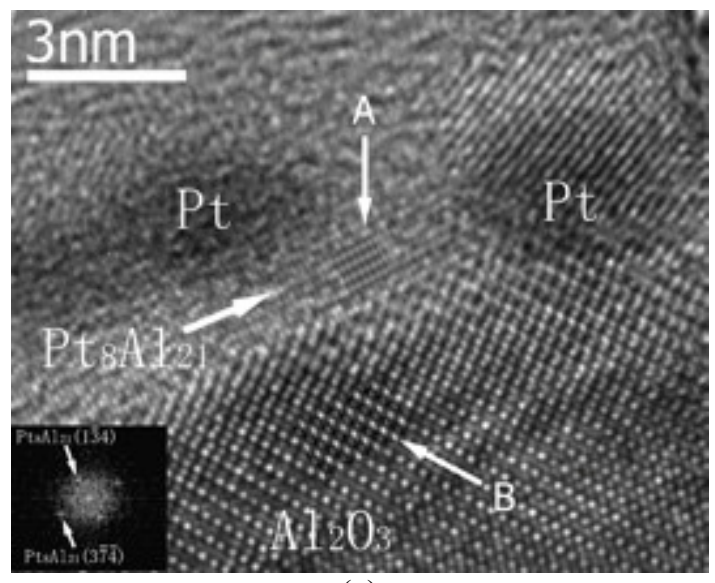

(a)

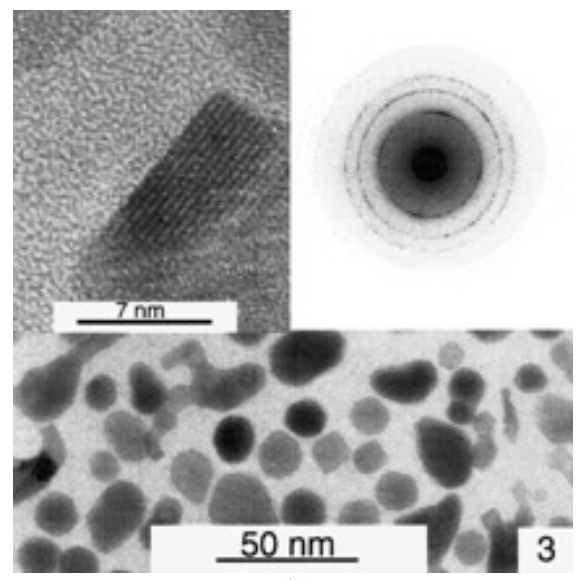

(b)

Figure 2-2 TEM image of Pt/Alumina in reduced atmosphere. (a) the intermetalic layer of $\mathrm{Pt}_{8} \mathrm{Al}_{21}$ formed in $10 \% \mathrm{H}_{2}, 1 \mathrm{hr}, 1073 \mathrm{~K}$ [15]. (b) $\mathrm{Pt}_{3} \mathrm{Al}$ layer fomed in Pure $\mathrm{H}_{2}, 1 \mathrm{hr}, 1073 \mathrm{~K}[14]$

For hydrogen spillover from a metal to an oxide, $\mathrm{H}_{2}$ usually dissociates on the metal into atomic $\mathrm{H}$, which then spills over onto the oxide [56]. Figure 2-3 depicts spillover by showing adsorption and activation of a diatomic molecule onto a surface. The adsorbed species can then move across the surface to the interface with the second surface, where the original diatomic cannot adsorb. From an energetic point of view, chemisorption on the original surface is exothermic accompanied by the surface diffusion, which may have small activation energy. Breaking the bond with the original absorbed surface and forming a new bond at the interface of the two phases is the endothermic reaction that must then be facilitated by an increase in entropy.

Spillover can take place from a metal to an oxide, from one metal to another, from one oxide to another, or from a metal oxide onto a metal. Spillover is not limited to the surface immediately adjacent to the activating surface; it can extend to other surfaces in contact with the accepting surface. As shown in Figure 2-3, a diatomic molecule first adsorbs onto a metal surface (dark region), and the activated species then spills over onto the support (white sphere), where it can diffuse to the interface with another surface (darkened sphere). The distances that the spilt-over species could transfer is not still clear, however Candau et al. [57] have reported 
spillover over millimeter or centimeter distances. Although $\mathrm{H}_{2}$ does not readily adsorb or dissociate on most oxide surfaces, $\mathrm{H}_{2}$ dissociates easily on metals like platinum. The resulting hydrogen atoms can then spill over onto oxide surfaces. Further, the spilt-over species can react with the oxide itself or even a second surface in contact with the accepting surface.
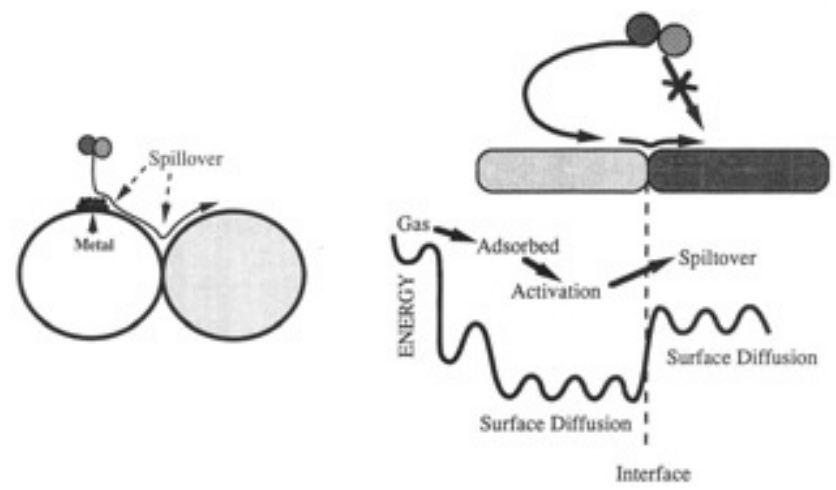

Figure 2-3 Schematic representation of spillover of a gaseous diatomic from an adsorbing surface onto a nonadsorbing surface, as well as its energy level diagram.

The bonding mechanism between platinum and alumina is mostly belived to be solidstate ceramic-metal diffusion bonding. However, despite the different reaction mechanisms and products that have already been reported for the $\mathrm{Pt} / \mathrm{Al}_{2} \mathrm{O}_{3}$ system, the exact bonding mechanism between them is not clear $[4,5,58,59]$. This ambiguity could be due to the fact that $\mathrm{Pt}-\mathrm{Al}_{2} \mathrm{O}_{3}$ or Pt-Al-O phase digrams are unknown or from the complicated behavior of metal and oxide at high temperatures. The first solid bonding of platinum/alumina system in a reduced atmosphere was reporetd by Allen and Borbidge [5]. They showed that bond strength increases by increasing time and temperature. They suggested that the best bonding was achieved just below the melting point of platinum and in the time of 10 hours or more. In addition, they showed that the application of force could enhance the bond strength.

Panfilov [4] used a plasma evaporation technique for joining platinum and alumina and reported the formation of the transition layer between the platinum and the alumina coating (30 
$\mu \mathrm{m}$ in depth). The transition layer contained small $(\sim 1 \mu \mathrm{m})$ and large $(\sim 20-50 \mu \mathrm{m})$ alumina inclusions and possessed a rough surface on the ceramic side. Additionally, the alumina particles penetrated into the platinum matrix during the sanding stage, but this treatment has not changed the mechanical behavior of the platinum either at room or elevated temperatures. However, the true nature of the transition layer and the bonding is not clear yet [4].

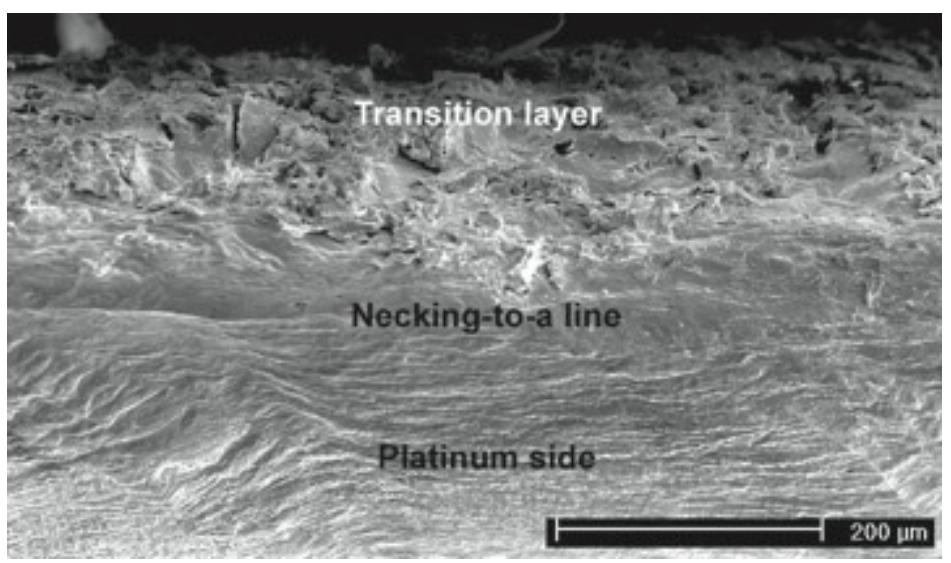

Figure 2-4 The transition layer between pt/alumina as observed by Panfilov [4]

De Graef et al. [60] used the platinum/alumina system with and without an $\mathrm{SiO}_{2}$ intermediate layer, for bonding in an air atmosphere. They showed that $\mathrm{SiO}_{2}$ could work as the liquid diffusion phase and enhanced the bonding between platinum and alumina by the assumption of the limited solubility of one of them or both. However, recently, Lu et al. [61] showed that the strength of direct bonding $\mathrm{Pt} / \mathrm{Al}_{2} \mathrm{O}_{3}$ depends basically on the crystal orientation of platinum and alumina. When the orientation is suitable for semi-coherency, the interface has a strong driving force to adopt direct metal-ceramic bonding; when the orientation is not suitable for coherency, then a weaker metal-glass-ceramic bond is favored since the covalent bonds in the glass and alumina are more conducive to mutual bonding. They showed that the orientation relationship of the interfacial planes is $(002)_{P t} / /(0 \overline{2} 2)_{\mathrm{Al}_{2} \mathrm{O}_{3}}$ [rhombohedral] or 
$(002)_{P t} / /(0 \overline{2} 22)_{A L_{2} O_{3}}$, [hexagonal]. Using the literature data and their experimental data, the lattice mismatch was calculated to be $\sim 15 \%$ and $11 \%$ respectively, which is about the size for a semicoherent interface threshold lattice mismatch (i.e. $~ 14 \%$ ). These orientations show good bonding without the presence of glass on the interface. Another study by Suppel et al. [6] proposed that the direct bonding of polycrystalline platinum to the (0001) single crystal sapphire demonstrates larger bonding strength in comparison to the bonding of platinum to other crystal orientation. Santala et al. [62] also observed that the platinum participates in sapphire in the following orientation relationships after annealing for 100 hours in $1600{ }^{\circ} \mathrm{C}: c(0001)_{\alpha} \|(111)_{P t}$; $(10 \overline{1} 0)_{\alpha} \|(1 \overline{1} 0)_{P t}$.

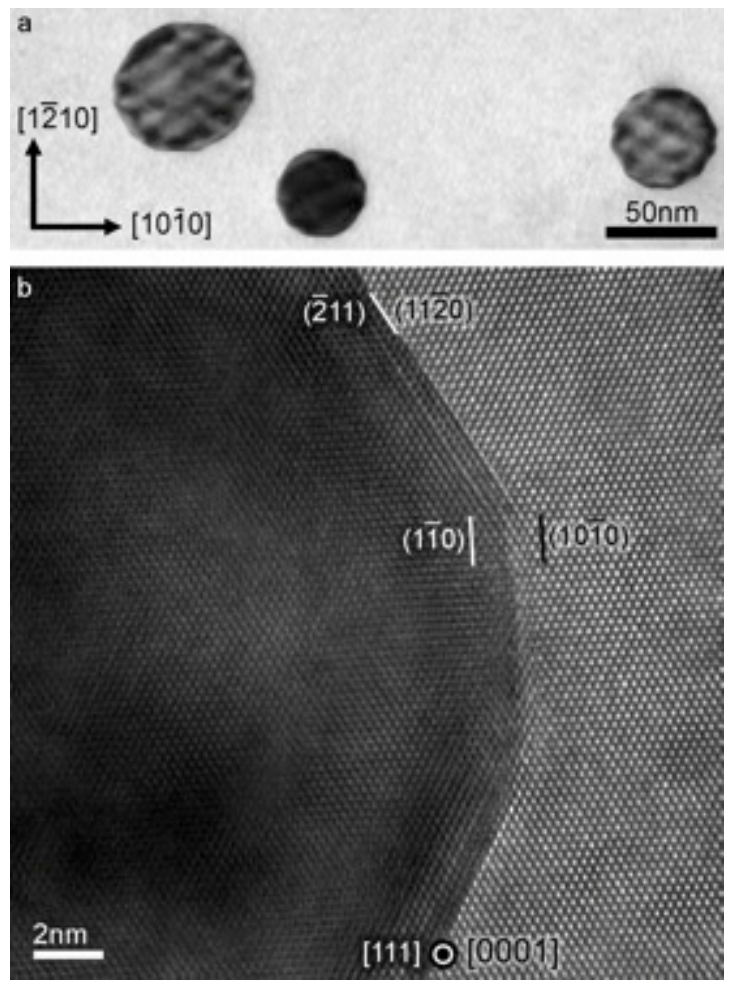

Figure 2-5 Platinum precipitates with $c(0001)_{\alpha}\left\|(111)_{P t} ;(10 \overline{10})_{\alpha}\right\|(1 \overline{10})_{P t}$ formed in sapphire after $100 \mathbf{h}$ at $1600{ }^{\circ} \mathbf{C}$, imaged by (a) bright field-TEM and (b) HRTEM[62] 


\subsubsection{Physical interaction in the metal/ceramic interface}

In 1961 Cole and Sommer [63] showed that in order to form metal/ceramic seals strong enough for most electronic applications, it was essential to form a dense metal/glass composite metallizing layer. They showed that the strength of the seal depended on the size of the alumina grains and firing temperature. Cole and Hynes [64], also indicated that the average grain size increased with successively higher firing temperatures. However, in large grain size metal surfaces was flooded with glass, the seals became weak because a intermediate layer for the metal/ceramic bond could not be created [65-67]. In general, it was shown [63-65, 68]that the metallizing variables affecting the strength of a metal-ceramic seal is determined by the extent of the glass migration into the metallizing layer. The most probable mechanism of glass migration is one of the capillary flow, in which the driving force is the surface tension of the liquid glass [66]. In the complete model to describe the glass migration, the ceramic and metal are assumed as two beds of powder. Capillary pressure of the molten glass in the pores is able to set up a pressure difference, which brings about a redistribution of the glass. Based on this model, the glass migration could be divided into three steps, as showed in figure 2-6.

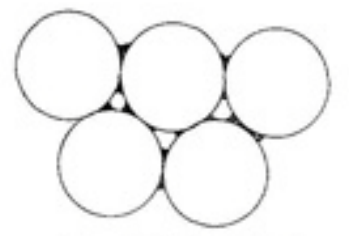

(a) Pendular stage

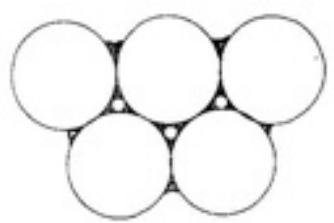

(b) Funicular stagz

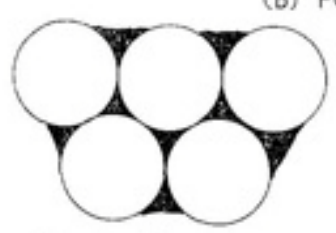

(c) Capillary stoge

Figure 2-6 Stages in the distribution of a liquid in a powder bed: (a) pendular stage; (b) funicular stage; (c) capillary stage. 
At low liquid concentrations, the liquid exists only as isolated wedges in the necks between the particles (pendular stage). As more liquid is added, the wedges build up until they just meet (funicular stage); at the last step, a small portion of liquid filled any hole and pores with capillary force (capillary stage). In the case of the sand bed and other powder beds in which there is a wide distribution of pore sizes, the three stages may co-exist in the partially saturated beds.

There will be two sets of suction pressures for each bed, depending upon whether liquid is flowing into the bed $\mathrm{P}(\mathrm{in})$ or whether it is flowing out, $\mathrm{P}(\mathrm{out})$. Thus, when the metallizing layer and the debased alumina are in contact at the metallizing temperature, glass will migrate from the alumina into the metallizing layer until the suction pressure of the metallizing layer PM (in) becomes equal to the suction pressure letting glass out PAl (out). In a favorable metallizing situation the two suction pressures will not become balanced until the metal layer is practically saturated with glass, while in an unfavorable situation they will become balanced when the metallizing layer is only partially saturated. The least favorable condition, PM (in) > PAl (out) only occurs at a very high temperature, and then as the sample cools down, PM (out) becomes less than PAl (in) and it is possible that some of the glass will drain out of the metallizing layer and go back into the alumina. The metallizing layer will, therefore, contain some glass but will be porous and weak.

Other than temperature, there are two major factors, which control the flow of glass; the glass migration could be caused by an increase in the volume of glass, which is produced by higher thermal expansion of the glass relative to the alumina. The values of PAl (out) and PAl (in) are assumed to decrease with increasing temperature as the glass expands more than the alumina and ultimately at a very high temperature (TF) it is possible for the glass to expand out 
of the alumina and flood the surface of the alumina as observed by Floyd [65].

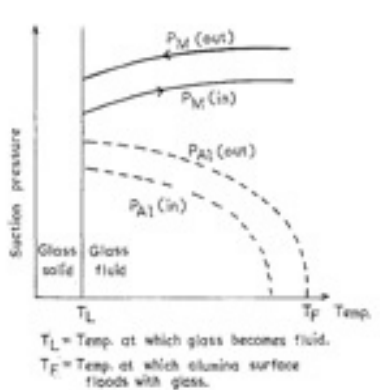

(a)

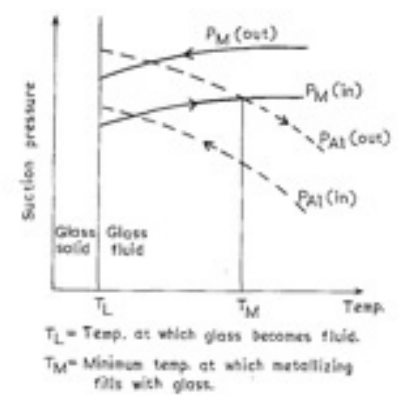

(b)

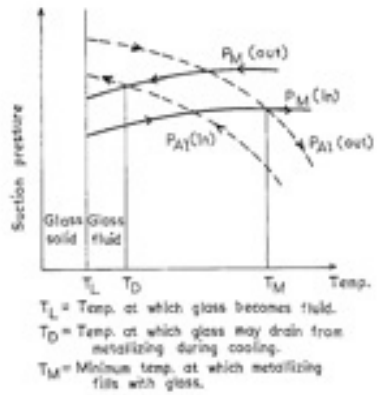

(c)

Figure 2-7 Different glass migration flow, (a) Ideal metallizing conditions, (b) Favorable metallizing conditions, (c) Unsatisfactory metallizing conditions (drainage).

Humidity of the atmosphere is also very important to determine the extent of glass migration into the metallization. In wet atmosphere, metal can form an oxide in high temperature, which could reduce the metal/glass contact angle. On the other hand, the glass/sapphire contact angles were less dependent on humidity and were always below 30 . This difference between contact angles increases the pressure by increasing the humidity. In contrast to the tungsten and other refractory metals, the results of platinum/glass contact angle showed that the contact angle does not change uniformly with temperature. It decreases with increasing the temperature up to about $1150^{\circ} \mathrm{C}$ and then increases [69]. This change of behavior can be due to the change of surface properties of platinum by temperature. Platinum surfaces can be oxidized by high temperature oxygen treatments in the 800 to $1100{ }^{\circ} \mathrm{K}$ temperature range [70]. This oxide is nonreactive below $1100^{\circ} \mathrm{K}$. Decomposition of $\mathrm{PtO}_{2}$ above $1150^{\circ} \mathrm{C}$ and desorption of oxygen from the bulk of platinum, decreases the interaction of the glass with surface of the platinum and then increases the contact angle. This change of behavior can change the effect of glass in platinum/ ceramic binding and also increases the chance of glass flooding on top of platinum. 


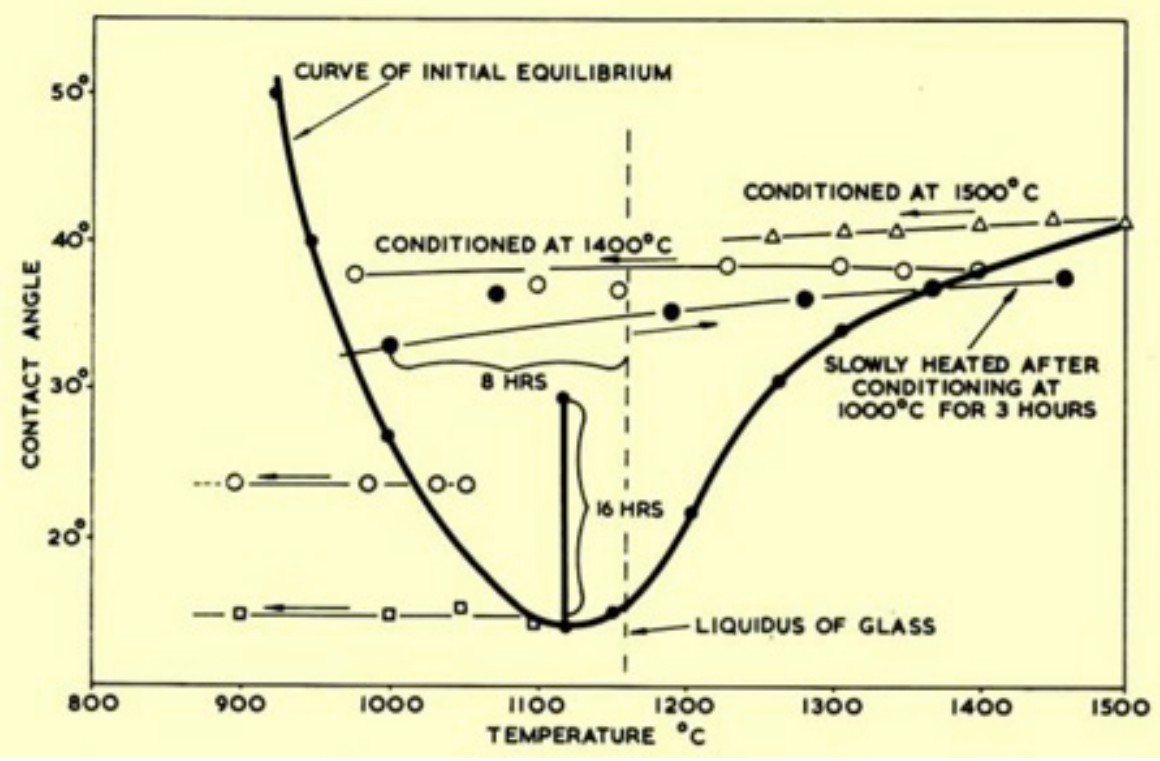

Figure 2-8 The platinum/glass contact angle in different temperature [69]

\subsection{Kinetics and thermodynamics of metal/ceramic interaction}

\subsubsection{Kinetics of metal/ceramic interaction}

The reaction kinetics of metal/oxide interface is controlled by the electric field, induced by electronic interaction, in particular, the long-range charge transfer at metal/oxide interfaces. It is therefore expected that there is an interplay between the electronic interaction and the chemical interaction in metal/oxide interface [2]. Such interplay originates from the strong coupling between the interfacial mass transport and charge transfer, which could be used to explain the kinetic of the reaction. The reaction results can be explained in the framework of Cabrera-Mott theory [71]. The Cabrera-Mott theory was first suggested to explain the oxidation of metal surfaces and formation of thin films by metal oxidation at low temperatures. In this theory, there are two important points to be considered, (1) electron transfer occurs between the 
surface adsorbed oxygen and metal and (2) the resulting electric field in the oxide layer can lower the barrier for ionic mobility.

The Cabrera-Mott theory can be generalized to be applied in the metal/oxide reaction by calculating the electric field at the interface, $E_{S}$, as a function of $E_{F}$ (Fermi energy) the positions in both metal and ceramic $[72,73]$. Interface reactions can be uniquely controlled by the relative positions of $E_{F}$ in both phases before establishing contact. A variation of these positions is directly coupled to the changes in the electric field at the interface and modifies the corresponding reaction rates. After contact between the metal and the oxide, charge transfer can establish electric field across the interface, subsequently affecting ion transport in the vicinity of the interface. An analytic expression of $E_{S}$ could be found in the work of Kingston and Neustadter [74].

The calculation demonstrates that $E_{S}$ changes with $E_{F}$ of the metal or the oxide monotonically and it could be used to predict the thermal stability of the metal/oxide interface semi-quantitatively. At relatively low temperatures, $E_{S}$ is a critical parameter that determines transport processes at the interface in the cases including the initiation of metal oxidation at metal/oxide interfaces and the encapsulation of metal nanoclusters by oxide supports. In each of the reactions mentioned above, the coupling between the mass transport and charge transfer may require different electronic configurations of the two contacting phases in order to favor the interface reaction. Lists of the transport processes in all those reactions are shown in table 2-2.

Table 2-2 Summary of reactions at solid-solid and solid-gas interfaces

\begin{tabular}{|c|c|c|c|}
\hline $\begin{array}{l}\text { Interface } \\
\text { reactions }\end{array}$ & $\begin{array}{l}\text { Contacting phases } \\
\mathrm{A} \| \mathrm{B}\end{array}$ & $\begin{array}{l}\text { Diffusing species \& } \\
\text { direction }\end{array}$ & $\begin{array}{l}E_{F} \text { arrangements favoring } \\
\text { reactions }\end{array}$ \\
\hline Redox reaction & Metal || Oxide & $\mathrm{O}^{2-}, \mathrm{A} \leftarrow \mathrm{B}$ & $E_{F}(\mathrm{~A})>E_{F}(\mathrm{~B})$ \\
\hline Encapsulation & Metal || Oxide & $\mathrm{Ti}_{\mathrm{i}}{ }^{\mathrm{n}+}, \mathrm{A} \leftarrow \mathrm{B}$ & $E_{F}(\mathrm{~A})<E_{F}(\mathrm{~B})$ \\
\hline $\begin{array}{l}\text { Surface } \\
\text { oxidation }\end{array}$ & Metal ॥ Gas & $\begin{array}{l}\mathrm{O}^{2-}, \mathrm{A} \leftarrow \mathrm{B} \text { or } \\
\mathrm{M}_{i}^{n+}, A \rightarrow \mathrm{B}\end{array}$ & $E_{F}(\mathrm{~A})>E_{F}(\mathrm{~B})$ \\
\hline
\end{tabular}


For example, in the case of encapsulation reaction [26], the metal should have a large work function and the oxide requires a high electron density or n-type doping like the $\mathrm{Pd} / \mathrm{TiO}_{2}$ system. The contact between the oxide and the metal will establish a positive space charges in the $\mathrm{TiO}_{2}$, which drive the outward diffusion of $\mathrm{Ti}_{i}{ }^{n+}$ but hinder the outward diffusion of oxygen anions $\left(\mathrm{O}-^{2}\right)$ at relatively low temperatures. Such a difference in the diffusion rates of Ti and $\mathrm{O}$ enables the enrichment of Ti at the surface. On the other hand, the outward diffusion of $\mathrm{Ti}_{i}{ }^{n+}$ will gradually weaken the positive space charges, which allows the outward diffusion of oxygen anions, thus favoring the formation of $\mathrm{TiO}_{x}(x<2)$ on the surface (see Figure 2-1B-D). Due to the difference of the surface energy of the $\mathrm{TiO}_{x}$ and the metal, $\mathrm{TiO}_{x}$ tends to cover the surface of the metal as clusters, resulting in encapsulation. At higher temperature, the diffusion of oxygen will be activated thermally and the decorating layer forms with the stoichiometric composition similar to $\mathrm{TiO}_{2}$.

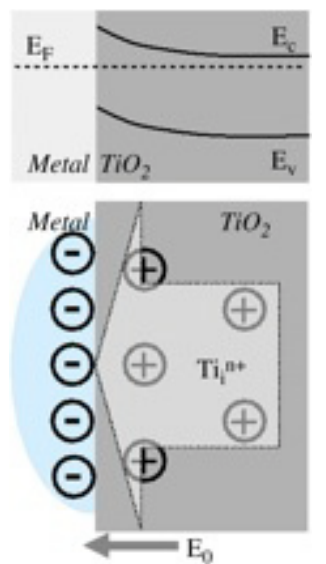

(a)

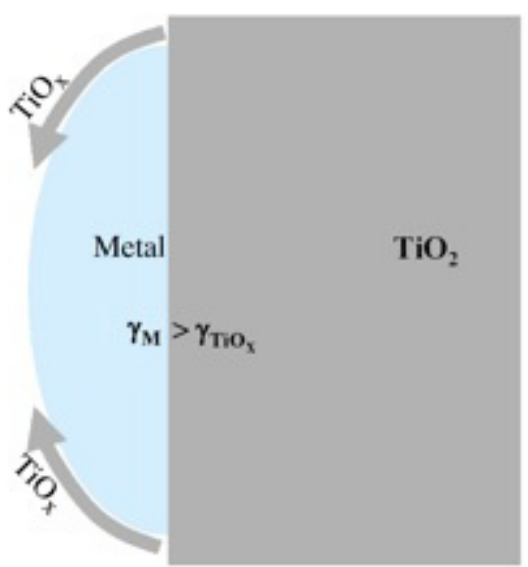

(b)

Figure 2-9 Schematic diagrams of kinetics and thermodynamics of encapsulation of metal with $\mathrm{TiO}_{2}$. (a) Energy bands of a metal/ $\mathrm{TiO}_{2}$ interface in the case of $E_{F}(\mathrm{Metal})<E_{F}\left(\mathrm{TiO}_{2}\right)$, which creates positive space charges at oxide surface regions to promote the outward diffusion of Ti interstitial ions, $\operatorname{Tin}^{+}(\mathrm{n}<4)$. (b) Mass migration of TiOx $(x<2)$ onto metal clusters driven by the minimization of surface energy of the whole system. 


\subsubsection{Thermodynamics of metal/ceramic interaction}

Thermodynamics can be used to find out if a chemical reaction is favorable. The classic thermodynamics may consider the bulk thermodynamic data, but for interface reactions, the influences of interface and the surface energy should be taken into consideration. In a simple

single oxide ceramic $\left(\mathrm{Me}^{\mathrm{II}} \mathrm{O}\right)$ in contact with a metal $\left(\mathrm{Me}^{\mathrm{I}}\right)$, the series of reaction could happen depending on the conditions. The following reactions can be written including the free energy $\left(\Delta \mathrm{G}_{0}\right)$ involved in the reactions:

$$
\begin{array}{lc}
\mathrm{Me}^{\mathrm{II}} \mathrm{O} \rightarrow \mathrm{Me}^{\mathrm{II}}+1 / 2 \mathrm{O}_{2} & \Delta G_{M e}{ }^{\mathrm{II} O} \\
\mathrm{H}_{2}+1 / 2 \mathrm{O}_{2} \rightarrow \mathrm{H}_{2} \mathrm{O} & \Delta G_{j} \\
\mathrm{Me}^{\mathrm{I}}+1 / 2 \mathrm{O}_{2} \rightarrow \mathrm{Me}^{\mathrm{I} O} & \Delta G_{M e}^{I} \mathrm{I} \\
\mathrm{Me}^{\mathrm{I}} \mathrm{O}+\mathrm{Me}^{\mathrm{I}} \rightarrow[\mathrm{O}] & \Delta G_{l} \\
\mathrm{Me}^{\mathrm{I} O}+\mathrm{Me}^{\mathrm{II}} \rightarrow\left[\mathrm{Me}^{\mathrm{I}} \mathrm{O}\right]_{\mathrm{Me}}^{\mathrm{II} O} & \Delta G_{m} \\
\mathrm{Me}^{\mathrm{I}}+\mathrm{Me}^{\mathrm{II}} \rightarrow\left[\mathrm{Me}^{\mathrm{II}}\right]_{\mathrm{Me}^{\mathrm{I}}} & \Delta G_{n}
\end{array}
$$

The component between the brackets [ ] is dissolved in the indexed phase. Reaction will occur if the total Gibb's free energy $\left(\Delta G_{R}=\Delta H-T \Delta S\right)$ of the relevant reactions is negative. In many ceramic-metal systems, a reaction is not expected if only the affinity of the metal to the non-metallic species of the ceramic is considered or if a reducing gas is involved in conditions that are not sufficient to reduce the ceramic. However, taking into account all the possible reactions, $\Delta \mathrm{G}_{\mathrm{R}}$ can become negative. For a large number of oxides and metal alloys the values for $\Delta \mathrm{H}$ are well known, but generally not for $\Delta \mathrm{S}$. Since in solid-state reactions, changes in entropy are negligible, the enthalpy changes for the reactions can be simply used 
for thermodynamic criteria to calculate the Gibbs free energy. Using this general description the temperature in which the reactions will happen, could be calculated.

For example, in the redux reaction system of $\mathrm{Nb} / \mathrm{Al}_{2} \mathrm{O}_{3}$ in vacuum in the range of temperature $1000-2000^{\circ} \mathrm{K}$, the condition can be written as:

$$
\begin{array}{lll}
1 / 3 \mathrm{Al}_{2} \mathrm{O}_{3} \rightarrow 2 / 3 \mathrm{Al}+1 / 2 \mathrm{O}_{2} & \Delta \mathrm{G}^{0}=562418.8-108.48 \mathrm{~T} \\
\mathrm{Nb}+1 / 2 \mathrm{O}_{2} \rightarrow \mathrm{NbO} & \Delta \mathrm{G}^{0}=-98860+20.31 \mathrm{~T} \\
\mathrm{NbO} & \rightarrow \quad[\mathrm{O}]_{\mathrm{Nb}}+\mathrm{Nb} & \Delta \mathrm{G}^{0}=7700+1.43 \mathrm{~T} \\
\mathrm{Nb}+2 / 3 \mathrm{Al} \rightarrow 2 / 3[\mathrm{Al}]_{\mathrm{Nb}} & \Delta \mathrm{G}^{0}=\Delta \mathrm{H}^{0}=-11470 \\
\hline 1 / 3 \mathrm{Al} 2 \mathrm{O} 3+\mathrm{Nb} \rightarrow{ }^{2 / 3}[\mathrm{Al}]_{\mathrm{Nb}}+[\mathrm{O}]_{\mathrm{Nb}} & \Delta \mathrm{G}_{\mathrm{R}}=31700-4.15 \mathrm{~T}
\end{array}
$$

Since it is a non-standard conditions reaction, the free energy of reaction should be calculated with Raoult's law:

$\Delta \mathrm{G}=\Delta \mathrm{G}_{\mathrm{R}}+5 / 3 \mathrm{RT} \operatorname{In} \mathrm{x}$

where $\mathrm{x}$ is the atom concentration of [O]Nb and consequently the concentration of [Al] $\mathrm{Nb}$ is $2 / 3$ $x$, yielding equilibrium oxygen partial pressure $\left(\mathrm{pO}_{2}\right)$ as:

$$
P_{O_{2}}=\exp \left(\frac{2 \Delta G_{s}^{0}}{R T}+2 \ln x\right)
$$

where $\Delta G_{s}^{0}$ is the Gibbs free energy for oxygen and could be derived from equations 8 and 9 . The Gibbs free energy and oxygen partial pressure can be calculated now as the function of temperature and $[\mathrm{O}] \mathrm{Nb}$, on the assumption that the solutes ( $\mathrm{Al}$ and $\mathrm{O}$ ) have no mutual effect. 
From these results it is clear that $\Delta \mathrm{G}$ depends on the oxygen concentration in the $\mathrm{Nb}$ and the oxygen partial pressure has to be extremely low for the reaction to proceed. The solubility of oxygen in $\mathrm{Nb}$ decreases with decreasing temperature, which means that $\mathrm{NbO}$ will form from a high-temperature saturated solid solution during cooling.

This type of thermodynamic calculation could also be used in alloy formation reaction. In the $\mathrm{Pt} / \mathrm{Al}_{2} \mathrm{O}_{3}$ system in hydrogen atmosphere, the Gibbs free energy of $\mathrm{PtO}_{2}$ reaction is positive but the enthalpy of the formation of the Pt-Al alloy is negative. If the heat of solution of $\mathrm{H}_{2}$ in platinum is neglected, the reaction equations for the standard condition in the temperature range $1000-2000^{\circ} \mathrm{K}$ are:

$$
\begin{array}{ccll}
1 / 3 \mathrm{Al} 2 \mathrm{O} 3 & \rightarrow & 2 / 3 \mathrm{Al}+1 / 2 \mathrm{O}_{2} & \Delta \mathrm{G}^{0}=562418.8-108.48 \mathrm{~T} \\
\mathrm{H} 2+1 / 2 \mathrm{O}_{2} & \rightarrow & \mathrm{H}_{2} \mathrm{O} & \Delta \mathrm{G}^{0}=-249659+57.11 \mathrm{~T} \\
2 \mathrm{Pt}+2 / 3 \mathrm{Al} & \rightarrow & 2 / 3 \mathrm{Pt}_{3} \mathrm{Al} & \Delta \mathrm{G}^{0}=\Delta \mathrm{H}=-127200 \\
\mathrm{Pt}+2 / 3 \mathrm{Al} & \rightarrow & 2 / 63 \mathrm{Pt}_{8} \mathrm{Al}_{21} & \Delta \mathrm{G}^{0}=\Delta \mathrm{H}=-19009.76 \\
& & \\
\hline 1 / 3 \mathrm{Al}_{2} \mathrm{O}_{3}+\mathrm{H}_{2}+2 \mathrm{Pt} \rightarrow & 2 / 3 \mathrm{Pt}_{3} \mathrm{Al}+\mathrm{H}_{2} \mathrm{O} & \Delta \mathrm{G}_{\mathrm{R}}=185559.8-51.37 \mathrm{~T} \\
1 / 3 \mathrm{Al}_{2} \mathrm{O}_{3}+\mathrm{H} 2+16 / 63 \mathrm{Pt} & \rightarrow 2 / 63 \mathrm{Pt}_{3} \mathrm{Al}+\mathrm{H}_{2} \mathrm{O} & \Delta \mathrm{G}_{\mathrm{R}}=293750-51.3 \mathrm{~T}
\end{array}
$$

Experimental data for $\mathrm{Pt} / \mathrm{Al}_{2} \mathrm{O}_{3}$ system are from Ref. $[55,75,76]$. For non-standard condition the Gibbs free energy is;

$$
\Delta G=\Delta G_{R}^{0}+R T\left(2 / 3 \ln \left[P t_{3} A l\right]+\ln \left(\frac{p H_{2} O}{p H_{2}}\right)\right)
$$


$\Delta G=\Delta G_{R}^{0}+R T\left(2 / 63 \ln \left[P t_{8} A l_{21}\right]+\ln \left(\frac{p H_{2} O}{p H_{2}}\right)\right)$

For $\Delta G=0$ and given values of $\left[\mathrm{Al}_{\mathrm{x}} \mathrm{Pt}_{\mathrm{y}}\right]$ the ratio $\mathrm{pH}_{2} / \mathrm{pH}_{2} \mathrm{O}$ is shown in figure 2-10. The combined influence of the entropy and of the heat of solution of $\mathrm{Al}$ in $\mathrm{Pt}$ on the required $\mathrm{pH} 2 /$ $\mathrm{pH}_{2} \mathrm{O}$ ratio for reaction is shown by the position of the lines relative to the line for the reduction of $\mathrm{Al}_{2} \mathrm{O}_{3}$ by $\mathrm{H}_{2}$ in the absence of $\mathrm{Pt}$ (eq. 24 and 15). It should be noted that, the reduction of the alumina occurs only at the metal interface and therefore does not affect the ceramic free surface.

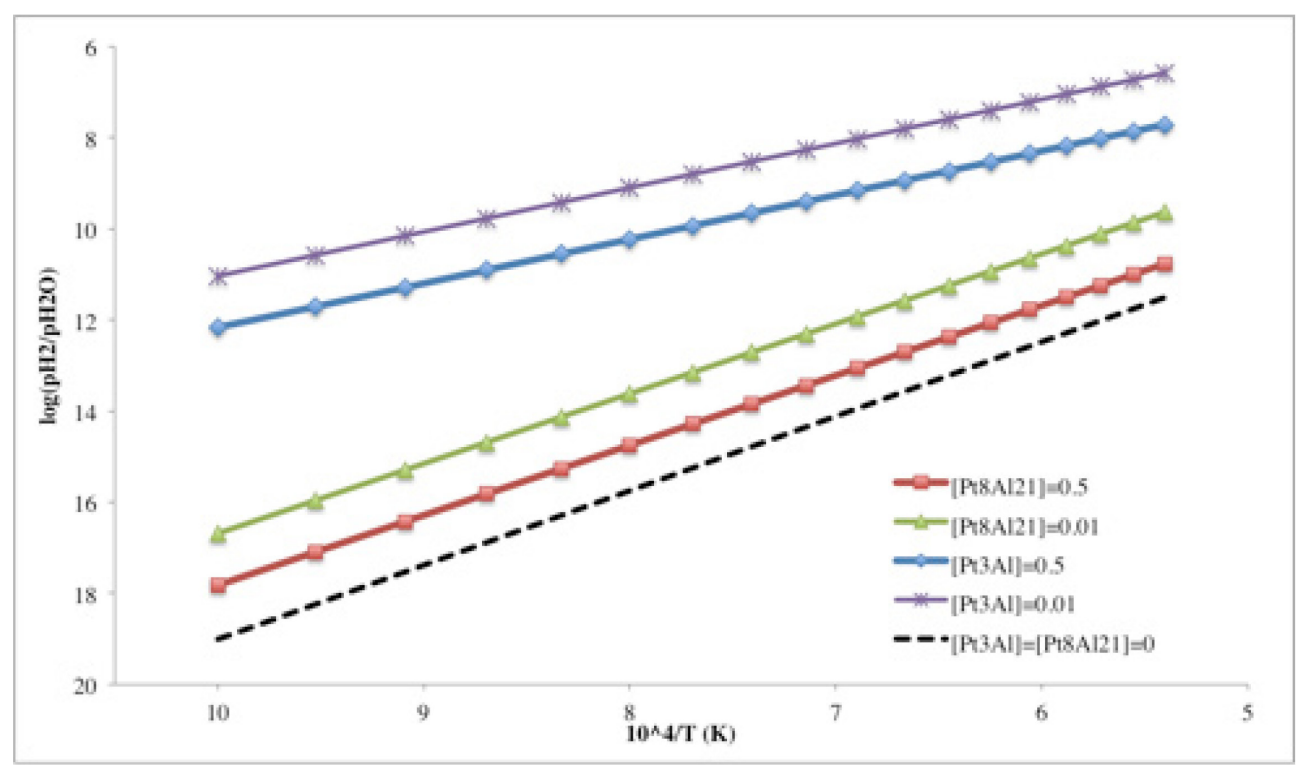

Figure 2-10 Reaction conditions for the $\mathrm{Pt}-\mathrm{Al}_{2} \mathrm{O}_{3}-\mathrm{H}_{2}$ system. Reaction occurs below the line of a given $\left[\mathrm{Al}_{\mathrm{x}} \mathrm{Pt}_{\mathrm{y}}\right]$.

It is clear from figure 2-10 that metals may be less reactive if they contain one or more components of the ceramic as impurities, depending on the heat of solution of ceramic components in the metal. Whether the reactions that are thermodynamically favorable really occur under the usual non- equilibrium conditions is based on reaction kinetics. The kinetics of 
the reaction play an important role here since reaction products could be observed only after prolonged heating.

In the above calculations, energies associated with surfaces and interfaces were not included. Interfacial energy is of general interest, because it is a fundamental thermodynamic property of interfaces. The ceramic-metal interfacial energy affects the contact angle, work of adhesion, and mechanical strength of the interface. In nanostructured metal/oxides system, the contribution from the surface and the interface energies to the total free energy could be significant and should be considered in the thermodynamic calculation. Several studies [26, 77, 78] showed that minimization of the surface energy of a system is one of the main driving forces for an encapsulation reaction, as shown in figure 2-9 b. In this case metals with high surface energy (e.g., Pt and Pd, but not $\mathrm{Au}, \mathrm{Cu}$ ) favor encapsulation and oxides with low surface energy such as $\mathrm{TiO}_{2}$ and $\mathrm{V}_{2} \mathrm{O}_{5}$, more easily undergo the encapsulation state than oxides with relatively high surface energies, such as $\mathrm{SiO}_{2}$ and $\mathrm{Al}_{2} \mathrm{O}_{3}[26,78]$.

In the case of a weak interaction between the metal film and the substrate, the minimization of the total Gibbs free energy due to surface energy gives rise to break down, decomposition and agglomeration of thin metallic films and in some cases the re-dispersion of the agglomerated particle. In the next section we will discuss the mechanism and thermodynamics of metal film instability.

\subsection{The instability of metal thin film}

At elevated temperature, the thin polished film is not as stable as in the room temperature. Atoms move at elevated temperature and surface grain grows wherever a grain boundary emerges to intersect the surface and in some cases this agglomerated films redisperse again. The 
morphological stability of a metal thin film on a ceramic material is conditioned by the aspect ratio, the interaction across the interface, and the tendency of the thin film to reduce its free surface energy. Two models have been proposed to explain the growth of dispersed metal particles on a surface of oxide ceramic. One is particle migration, which postulates the migration, collision, and coalescence of nanoparticles [79]. Another alternative is the molecular migration model where the growth occurs by inter-particle transport of metal atoms or molecular species $[27,29]$. The latter mechanism is often referred to as Ostwald ripening [80]. It has been showed $[33,81-84]$ that either particle migration or atomic migration is the sintering mechanism according to the support used and the interaction between metal and oxide support. Baker et al. $[81,82]$ evaluated the strength of metal/oxide interaction for different systems and showed that the sintering mechanism is the atomic migration when the interaction is strong but it changes to the particle migration when the interaction is weak. Straguzzi et al. [83] showed that the number of the interaction points increases by increasing the surface area of the particle. For small surface area particles the interaction is probably weak and the resistance to migration is low, so they can migrate on the surface of oxide and sinter upon collision; while for high surface area particles, the growth can occur only through the atomic migration.

In the case of metal/oxide interface, two kinds of re-dispersion are distinguished [32]. In the first kind, no wetting angle can exist between crystallites and substrate; in the second kind a wetting angle is possible, but a two-dimensional fluid coexists with the crystallites. At high temperatures, platinum starts to oxidize. In case of $\mathrm{Ni} / \mathrm{Al}_{2} \mathrm{O}_{3}$, results showed that $\mathrm{NiO}$ and $\mathrm{NiAl}_{2} \mathrm{O}_{4}$ are present after heating and $\mathrm{NiO}$ almost transformed completely to $\mathrm{NiAl}_{2} \mathrm{O}_{4}$ after 2 hours. The electronic structure of alumina suggests that the interactions between metals and oxide surfaces in low temperature are dominated by either interfacial bonding or metal 
polarization effect, yet some researches reported the observation of the formation of platinum aluminate structure in temperature as low as $650^{\circ} \mathrm{K}$. This oxide starts to spread from the platinum particles over the surface of the alumina by extending what are referred to as their feet. If several particles are close enough, the spreading films meet each other and merge into a patch of relatively thick film, which joins the particles. In the $\mathrm{Ni} / \mathrm{Al}_{2} \mathrm{O}_{3}$ system, after 30 min of heating the foot starts to detach from the body, probably due to the strain buildup at the junction of foot and main body of particle. After about 2 hours, the feet of the particles continue to spread over the surface of alumina to form aluminate and create a contiguous film, undetectable by electron microscopy that covers the entire substrate. After about 14 hours of heating in steam, the particles achieve their equilibrium angle and coexist with the undetectable film [7].

In the first step of heating, the foot tends to spread due to the large spreading force acting on the thin film, while the main body of the particle tends to contract due to the formation of aluminate. Such a detachment occurs only if the stress generated is large enough, which is not the case for all particles [7]. The complex formed at the substrate-metal interface has lower wettability in comparison to the metal oxide; in addition, it has a structure that induces strain in the metal energy that is relaxed in part by the fracture of the crystallite $[51,85,86]$. It can also diffuse into the substrate and break the alumina substrate [87]. Wang and Schmidt [86] developed a void model to described the particle splitting in the sintering process. At the metalmetal oxide interface, cations form and diffuse through the oxide film along with electrons to the metal oxide-oxygen interface. Oxide thus grows at the free surface of the oxide film with metal ions coming from the underlying metal. On a flat surface the oxide can recede as the metal leaves the interface, while on a curved surface a void is necessarily created unless the oxide film can break up and collapse continuously on the receding metal. An identical situation may occur in 
oxidation of a small metal particle. Metal will be transported from the metal-metal oxide interface in forming the new oxide, and this must create a void at the interface, which will stop the further growth. Growth can only continue if a metal transport path exists from the metal particle to the metal oxide layer.

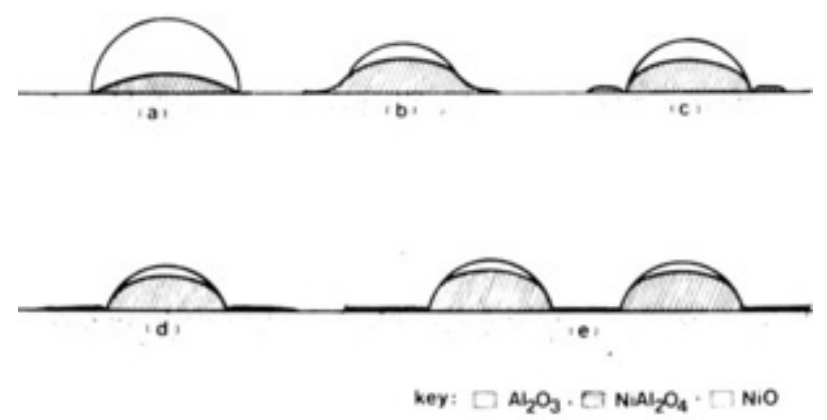

Figure 2-11 Schematic representation of re-dispersion and sintering (a) nonwetting nickel particle in steam before feet formation, (b) the formation of feet on heating in steam, (c) the detachment of the feet from the main body on further heating in steam, (d) the spreading of the feet to form thick films, and (e) formation of a thin surface film

\subsubsection{Thermodynamics of thin film instability}

In addition to the approach mentioned above, the stability of thin films in high temperature could also be studied in the context of thermal grooving theory [88-93]. At elevated temperatures, the thin film is not as stable as in the room temperature. Atoms move at elevated temperatures and surface grain grows wherever a grain boundary emerges to intersect the surface. One particularly interesting and general problem relates to the stability of the film against shape changes, which tend to break up the continuous film into a group of islands. Instability of thin films is mainly due to grain boundary grooving and grain boundary vortices caused by capillary forces. In the idealized case, Mullins [88] showed that the evolution of a surface driven by 
capillary forces may be described in terms of velocity $V_{n}$, of the outward surface. In the case that dominant transport mechanism is surface diffusion, the velocity can be written as $V_{n}=B \nabla_{s}^{2} \kappa$

where $\kappa$ is the local curvature of the surface; and $V$ is a surface Laplacian and $B=D_{s} \gamma_{\nu} \Omega^{2} v / k T$ is the normalized diffusivity. The normalized diffusivity is a function of $D_{s}$, the surface selfdiffusion coefficient, $\gamma_{v}$, the film-vapor surface energy, $\Omega$, the atomic volume, $v$, the number of diffusing atoms per unit surface area, and $k T$, the thermal energy. For a sinusoidal perturbation, $\Phi_{q}(t)=A(t) \sin (q x)$, the difference between the energy of the perturbed system and that of the unperturbed system (i.e., flat film of uniform thickness) is given by

$$
\Delta E=\gamma_{\nu}\left\{\int_{S}\left[1+\left(h^{\prime}\right)^{2}\right]^{1 / 2} d s-\int_{S_{T}} d s\right\}+\gamma_{s}\left\{\int_{S^{\prime}} d s-\int_{S_{T}} d s\right\}
$$

Where $\gamma_{S}$ is the film-substrate surface energy and $\left(h^{\prime}\right)^{2}=(\partial h / \partial x)^{2}+(\partial h / \partial y)^{2}$, where the local film thickness $h(x, y)$ is initially parallel to the $x-y$ plane. $S_{T}$ and $S^{\prime}$ represent the total area of the substrate and the area of the substrate covered with the film, respectively.

In real case, unlike the ideal thin film considered by Mullins, microstructures are composed of interconnecting grain boundaries. The thin film consists of several grains. Each grain boundary interacts via surface diffusion with other grain boundaries. In addition to that, the properties of the vertex line where three-grain boundaries meet may be different than those of the grain boundaries in isolation. In a simple case grain boundary vortices can be considered as a periodic arrangement of a series of circular holes extending from the vapor down to the substrate, and grain boundary grooving could be considered as periodic arrangement of a series of island. 


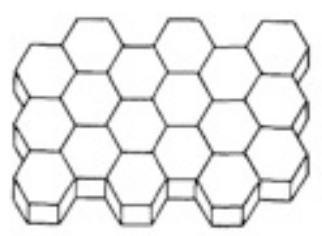

a

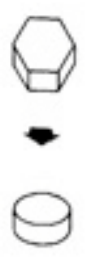

b

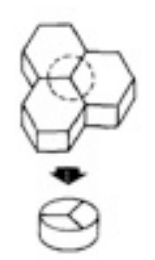

c

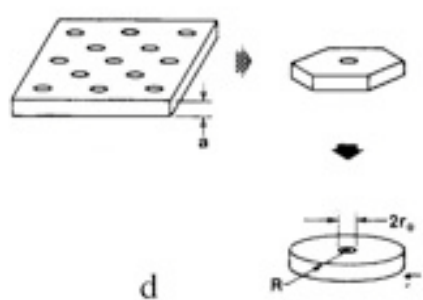

d

Figure 2-12 A symmetric arrangement of grains in a thin film (a). The grains extend from the free surface down to the substrate. There are two equivalent unit cells in this structure. (b) One grain completely surrounded by grain boundaries. (c) The grain boundary vertex where three grain boundaries meet. (d) A thin film containing an array of holes with radius, which could be used to model the grain boundary vertices.

In the first case, Srolovitz and Safran [90] showed that, with the small slope

approximation equation can be written as

$$
\Delta E=2 \pi \gamma_{0} \int_{r_{0}}^{R}\left[1+\left(h^{\prime}\right)^{2}\right]^{1 / 2} r d r+2 \pi \gamma_{s} \int_{r_{0}}^{R} r d r-\left(\gamma_{s}+\gamma_{\nu}\right) \pi R^{2} \approx\left[\gamma \int_{r_{0}}^{R}\left(h^{\prime}\right)^{2} r d r-(1+\gamma) r_{0}^{2}\right] \pi \gamma_{s}
$$

where $\gamma=\gamma_{v} / \gamma_{S}$. When the slopes are large the small slope approximation (i.e. $\left[1+\left(h^{\prime}\right)^{2}\right]^{1 / 2}=1+1 / 2$ $\left.\left(h^{\prime}\right)^{2}\right)$ leads to the results which should be both qualitatively and semi-quantitatively valid. By the help of the minimization process, equation 24 yields to

$$
\frac{\Delta E}{\pi a^{2} \gamma_{v}}=\frac{-4}{3+4 \ln \rho-4 \rho^{2}+\rho^{4}}-\beta \rho^{2}
$$

where $\rho=r_{0} / R$ and $\beta=(R / a)^{2}\left(1+\gamma_{s} / \gamma_{v}\right)$. In case of grain boundary vertex Srolovitz and Safran added the grain boundary energy to equation 23 , however the final equation did not have complete analytical equation.

$$
\Delta E=2 \pi \gamma_{v} \int_{r_{0}}^{R}\left[1+\left(h^{\prime}\right)^{2}\right]^{1 / 2} r d r+3 \gamma_{G} \int_{r_{0}}^{R} h d r-\gamma_{v} \pi R^{2}-3 \gamma_{G} R a
$$

The minimum value of $\rho=r_{0} / R$ can be determined below which no equilibrium grain boundary vertex pit exists, and is written as 
$\rho_{\min }=\frac{\sqrt{\Gamma}-\Gamma}{1-\Gamma}$

where $\Gamma=3 \gamma_{G} / 2 \pi \gamma_{\nu}$. Srolovitz and Safran argued that, since $\rho_{m}$ would be expected to be in the range of 0.1 to 0.5 , the hole due to the grain boundary vertex pit is expected to grow. These results suggest that polycrystalline films are unstable to hole formation and growth leading to film rupture due to the presence of grain boundary vertices.

In calculating the energetics of the islands, the same procedure used for holes can be developed. Applying equation 23 to the island geometry yields the difference in energy between the array of islands and the uniform film and can be shown as:

$$
\Delta E=\gamma_{v} 2 \pi \int_{0}^{\gamma_{0}}\left[1+\left(h^{\prime}\right)^{2}\right]^{1 / 2} d s+\gamma_{s} \int_{0}^{r_{0}} r d r-\left(\gamma_{v}+\gamma_{s}\right) \pi R^{2}
$$

Which yields to the:

$$
\frac{\Delta E}{\pi a^{2} \gamma_{\nu}}=\frac{4}{\rho^{4}}+\beta\left(\rho^{2}-1\right)
$$

Where $a$ is the film thickness and $\beta$ has the same meaning as before. Calculating the minimum of $\Delta \mathrm{E}$ for different $\beta$, shows that the minimum happens at $\rho=8^{1 / 6} / \beta$. Increasing $\beta$ moves the minimum to smaller $p$. Their results showed that, for $\beta>27$, the minimum value of $\Delta E$ is less than zero, and hence, the island is stable with respect to the film. For $\beta<8$, the minimum in $\Delta E$ occurs at $\rho>1$. Since $\rho$ cannot be greater than 1 , this indicates that the array of islands will touch and reestablish the continuous film. For $8<\beta<27$, the islands have a minimum energy shape and size, but the array of islands is in metastable equilibrium with respect to the continuous film. In a real grain structure, the minimum surface energy shape of each grain is a spherical cap, which is consistent with the parabolic structure of the islands from equation 28. This cap 
meets the grain boundary at an equilibrium angle. The equilibrium angle $\theta$, as defined in figure $2-13$, is given by $\theta=\sin ^{1}\left(\gamma_{G} / 2 \gamma_{v}\right)$. Based on the equilibrium angle, the depth that the groove that will intercept the substrate could be calculated as:

$$
\left(\frac{R}{a}\right) \geq \frac{3 \sin ^{3} \theta}{2-3 \cos \theta+\cos ^{3} \theta}
$$

Similar to an island structure, it can be shown that for $\beta>27$, the grain boundary groove intersects the substrate and the bumps will contract to form separated islands.

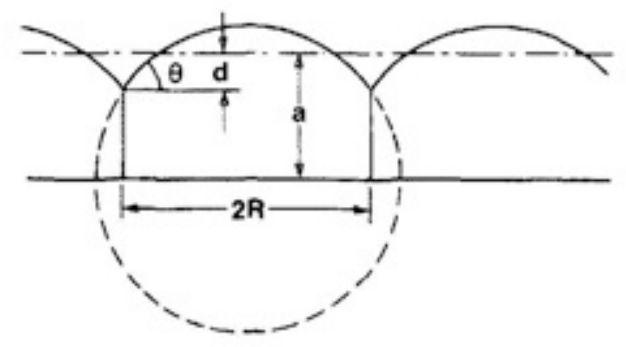

Figure 2-13 The equilibrium grain boundary groove configuration for a circular cross-section grain. The shape of the free surface is a spherical cap which intersects the grain boundary at an angle $\theta$, the grain diameter is $2 \mathrm{R}$, the film thickness is a, and the equilibrium groove depth is $\mathbf{d}$.

Employing the presented calculation showed that holes in thin films will grow provided they are larger than a critical value which is a function of the inter-hole spacing and the ratio of two interfacial parameters, as embodied in the composite parameter. For large value of $\beta$, the film pinches off to form islands, and the islands will be stable. However, if the film goes through a temperature change process to change $\beta$, a transition from islands back to holes is possible. Srolovitz and Safran [90] showed that $\beta$ for the island to hole transition is between 8 and 27. This suggests that there is some hysteresis in the hole-island-hole transition cycle.

Based on maximum depth calculation from equation 30, the maximum grain size for which the grain boundary groove will eventually hit the substrate and hence ruptures the film could be 
calculated. In most cases, in addition to grain boundaries, the pit associated with a grain boundary vertex will extend all the way down to the substrate. The holes associated with grain boundary vertices grow. These holes are more detrimental to film integrity and more difficult to protect against than the grain boundary grooves.

\subsubsection{Thermodynamics of thin film sintering and re-dispersion}

Sintering and re-dispersion process could also be explained thermodynamically in terms of wetting $[7,32,94,95]$ and the conditions that sintering by migration of crystallites or sintering by Ostwald ripening could be identified. When a thick film is transferred from a large reservoir to a uniform substrate, which means that the range of the interaction forces between one atom and the substrate is much smaller than the film thickness. The specific free energy of formation is given by:

$\sigma_{\infty}=\sigma_{m g}+\sigma_{m s}-\sigma_{g s}$

If $\mathrm{h}$ indicates the thickness of the film, the $\sigma_{\infty}$ is the free energy of the film with $\mathrm{h}$ thickness. If the $\sigma_{\infty}<0$ the film is stable and it wets the substrate. The interfacial free energy between film and substrate is given by,

$\sigma_{m s}=\sigma_{m}+\sigma_{s}-U_{m s}+U_{m s}^{s}$

where $U_{m s}$ is the interaction energy between the metal and substrate and $U_{m s}^{s}$ is the strain energy per unit area due to the mismatch of the two lattices. Substituting equation 32 into equation 31 and considering that $\sigma_{\mathrm{m}} \approx \sigma_{\mathrm{mg}}$ and $\sigma_{\mathrm{s}} \approx \sigma_{\mathrm{sg}}$, the free energy of the film formation can be approximated as:

$\sigma_{\infty}=2 \sigma_{m g}-U_{m s}+U_{m s}^{s}$ 
At the wetting condition of $\sigma_{\infty<0}$ or when $2 \sigma_{m g}<U_{m s}+U_{m s .}^{s}$, the film spreads over the substrate because this causes a decrease of the free energy of the system. When the film is not stable thermodynamically, it coalesces into islands. The island forms the angle $\theta$ with the substrate based on Young's equation.

Same kind of formulation can be used to compare the driving forces for the spreading of the aluminate phase or of the oxide phase on alumina, and the free energies can be written respectively as

$\sigma_{\text {oxide/alumina }}=2 \sigma_{\text {oxide }}-U_{\text {oxide-alumina }}+U_{\text {oxide-alumina }}^{s}$

$\sigma_{\text {aluminate/alumina }}=2 \sigma_{\text {aluminate }}-U_{\text {aluminate-alumina }}+U_{\text {aluminate-alumina }}^{s}$

In the case of $\mathrm{Ni} / \mathrm{Al}_{2} \mathrm{O}_{3}$, no data is available for surface energy of aluminate and oxide though it is reasonable to consider that $\sigma_{\text {aluminate }}$ and $\sigma_{\text {oxide }}$ are of the same order of magnitude. The lattice parameter of the alumina and aluminate are in the same order and $\mathrm{NiO}$ is almost half of them, which indicates the greater strain energy for $\mathrm{NiO}$ on alumina than for the aluminate on alumina. However, because $\mathrm{NiO}$ can interact chemically with alumina to form aluminate and no such strong chemical interactions exists between aluminate and alumina, one has $U_{\text {oxide-alumina }}>>$ $U_{\text {aluminate-alumina }}$, which suggest that $\sigma_{\text {oxide/alumina }}<\sigma_{\text {aluminate/alumina }}$, and oxide over alumina is more stable than aluminate on alumina. 


\section{Multilayer Ceramic technology}

\subsection{Introduction}

Multilayer ceramics represent a number of technologies that are capable of producing highdensity electronic substrates with highly desirable properties. The development of multilayer ceramics was first demonstrated in the preparation of capacitors in the late 1940s [96]. Over the following 10 years, the techniques necessary to produce multilayer high-temperature cofired ceramics (HTCC) substrates were developed. This technology has been used and is still in use for a wide range of single-chip packaging solutions as well as for multilayer substrates used in high-density electronic modules. The benefits of this technology include low electrical losses, very high interconnect density, stability at high temperatures, and the ability to easily include three-dimensional structures such as inductors and ceramic microelectro-mechanical system (CMEMS) devices. In addition, these materials can be intrinsically hermetic in nature and offer thermal conductivities as much as 100 times greater than traditional polymeric substrates.

The multilayer ceramic process is fundamentally a parallel process in which each layer is fabricated separately and then combined to form a device, as shown in figure 2-1. Each layer begins as a roll of glass and/or ceramic particles in a polymer matrix, which has been casted as tape to a particular thickness on a plastic film. After cutting the tape to size, each layer is punched or laser-cut to form the holes (called vias) and any desired cavities. The vias are filled with conductive ink. In general, each tape layer contains a unique set of printed electrical traces on its surface, as well as vias through that given layer. Each layer is submitted to a screenprinting process to create the interconnects on the surface of the tape layers. Throughout careful design of this combination of layers, traces, and vias, a three-dimensional block of ceramic is created with embedded electrical circuitry. At this point in the process, the layers are stacked up 
and aligned to each other. Once aligned, the assembly is laminated to bond the polymer components of adjacent layers. This stack of layers is still a flexible substrate that contains significant amounts of polymeric material. In the firing process, the polymeric component is burned off in a furnace as the first step. The polymer component is converted to gas and vented from the furnace, leaving behind only the ceramic or glass as well as the metal traces and vias. This assembly is fired to sinter the ceramic or glass into a dense ceramic body that contains the desired metal interconnections [97].

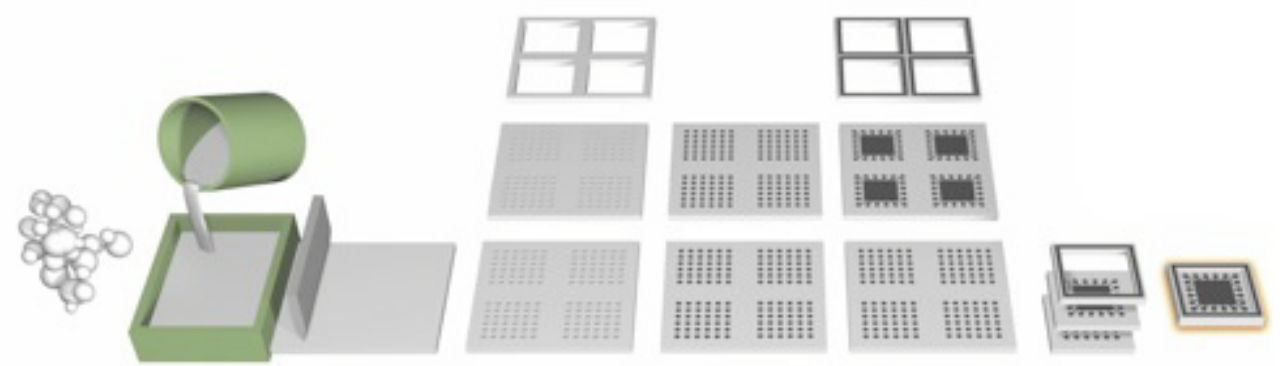

Figure 3-1The process of multilayer ceramic technology

\subsection{Materials}

\subsubsection{Ceramic tape}

High Temperature Cofired Ceramic (HTCC) is a term to describe ceramic substrates that are consolidated at temperatures above about $1000^{\circ} \mathrm{C}$. This definition includes aluminum oxide, aluminum nitride (AlN), and a variety of other under development or barley-used materials. HTCC alumina tapes have been commonly used in electronic packages for decades due to their benefits, cost and existing manufacturing infrastructure. The benefits include electrical performance, mechanical stability, thermal properties and compatibility with existing microelectronics processes. HTCC is mechanically very stable compared to other ceramics or 
organic packagings. The Young's Modulus for $92 \%$ alumina is about $275 \mathrm{GPa}$ compared to plastics, which are 50 to 100 times less. A very important benefit of HTCC is the ability to achieve hermetic IC packages. This is an important benefit for different industries especially the medical market [98-100]. However, some applications were hard to fit in the HTCC technology because of the intrinsically low conductivity of refractory conductors that are able to withstand the high firing temperatures that HTCC requires. An extension of this technology is Low Temperature Cofired Ceramic (LTCC). LTCC was developed in the early 1980s, in part for applications that suffered from the high conduction loss of HTCC products [101-104].

LTCC in some way was evolved from HTCC with the purpose of achieving low loss, high speed, and high-density packaging with lower firing material. Lower firing temperature allows for the incorporation of non-refractory metals such as silver, gold, and copper. These materials offer far superior electrical conductivities, and therefore LTCC can, in general, offer superior high frequency electrical performance in comparison to HTCC. Because of these properties, LTCC has been used to produce Multichip Ceramic Modules (MCM-C), sensors and actuators [105]. However, this improvement is not without its limitations; LTCC materials are glass-ceramic compositions with inferior mechanical and thermal properties when compared to HTCC. This reduced strength and reduced thermal performance are intrinsically tied to the compositions of the crystallizable LTCC materials or the sintering additives that are added to the alumina-glass LTCC composites. In addition to that, HTCC materials are mostly biocompatible and used for bio application, while LTCC materials are not proven to be biocompatible. As a result, a trade-off is required to select the material that best matches the requirements of a given application. 
HTCC and LTCC offer a number of compelling advantages over traditional thick-film, organic, and other packaging options. In general, HTCC substrates exhibit high mechanical strength, high thermal conductivity, consistent and attractive electrical performance, hermeticity, refractory metallization, and reliable brazing technologies. LTCC offers many of the same features but with reduced mechanical properties and improved electrical properties [106].

\subsection{Cofired inks}

Both noble and refractory metals ink are used in multilayer HTCC ceramics. Noble metals (Pt and $\mathrm{Pd}$ ) are relatively expensive but may be fired in air atmosphere, while, refractory metals (Wand Mo), must be fired in a reducing atmosphere to prevent oxidation. On the other hand, gold, silver, and copper are the most dominant inks for the LTCC technology. These metals are compatible with the low-firing temperatures of the LTCC systems and offer superior electrical conductivity. Gold finds major use in aerospace and high-reliability applications due to its stability and oxidation resistance. Silver is much lower in cost and can be fired in air, so it is very attractive for more cost-sensitive markets. Copper is also inexpensive but because of its strong affinity for oxygen, special inert firing atmospheres are required[97, 99, 100, 104].

\subsection{Tape handling and casting}

Casting and handling tape for multilayer ceramic application is the well known traditional process that is used for more than 50 years. Multilayer ceramic tapes use many of processes that have been used for more than 50 years to produce thick-film circuits and components [107, 108]. Because of the fragile nature of the tape layers in green state, some special precautions are necessary in their handling process. The five general areas of concern are humidity control, 
temperature control, reduction of particulate matter, and static control, as well as support and handling of the fragile tape layers.

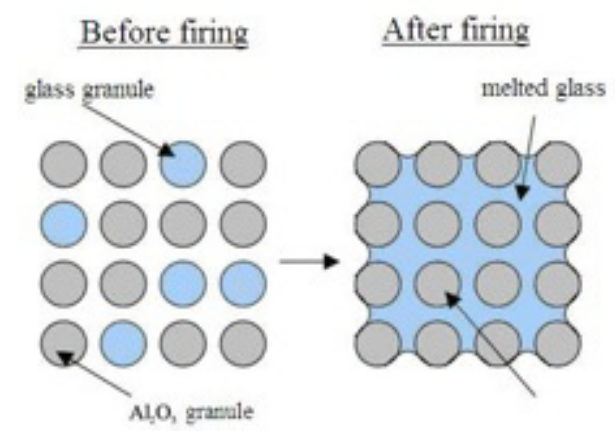

Figure 3-2 Sintering mechanism of the HTCC tape

Ceramic substrates are typically composites of crystalline and noncrystalline phases. The composition of the substrate is, of course, a function of the inorganic (and organic) components incorporated as raw materials. The first HTCC tape was produced with $92 \%$ alumina, while different composition of alumina from $90-99 \%$ are currently using as the commercial HTCC tapes. In the most commercial HTCC products, the three main inorganic components are alumina, talc and clay. Clay and talc are used as the glass phase and decrease the sintering temperature by creating liquid phase sintering. Some other additives like chromium oxide or refractory metals (i.e. W, Mo and Ti) also could be added to the formulation to control the color and opacity of the final tape. Most of the time this colorification and opacification does not have any functional benefit, although it eliminates the visibility of subsurface metallization.

Particle size is an important element in the performance of an HTCC system. Fine particle size promotes rapid densification and can be an effective means of lowering the sintering temperature or time spent at temperature; they are often difficult to disperse effectively. Agglomerates and aggregates function as large, primary particles, exhibiting correspondingly large pores and voids. This coarse microstructure leads to a net reduction in sintering efficiency. 
Prior to mixing, the classification of powders has been employed as a means of rendering a more uniform particle size distribution, or to remove large agglomerates.

In the tape casting process, inorganic powders are mixed with solvents, binders, dispersants, plasticizers, deflocculants, and other additives. At the first step, the slurry of ceramic powders, solvent, and dispersant is milled for $12-24 \mathrm{~h}$ until the desired degree of dispersion, reflected in the slip viscosity, is achieved. In the second stage of slip preparation, the remaining organic components are added and milled for additional $12-24$ hours. At the end, the milled compound is casted onto the polymeric carrier with desired thickness.

\subsection{Via and cavity formation}

The first process after preparation of the tape, is typically via and cavity formation. In this process vias, cavities and channels are punched or cut into the tape. These are to form a wide variety of unique electrical and mechanical features within a substrate and to allow electrical connections between layers in the vertical axis of the substrate. Laser cutting and mechanical punching are the two major approaches that are used for via and cavity formation[109].

By far, mechanical punching is the most popular method for via formation due to the highspeed nature of this process as well as high quality of the resulting vias. Laser cutting of tape has its advantages in the formation of channels or cavities with complex shapes. However lasers generally do not cut vias as cleanly as a mechanical punch, and vias are conical in shape when they are cut with laser. This is due to small spot size of the laser and its shallow depth of focus. As the laser starts to cut through the ceramic, it begins to defocus slightly, which results in the conical shape of the via. In addition, the ablation process tends to leave debris on the tape's surface. This material consists of the tape material that was not completely ablated. 
In contrast to the laser, mechanical punches must use custom die or be programmed to nibble away the desired structure. Mechanical punching methods can be separated into two broad categories: hard tooling and soft tooling. Hard tooling consists of a designated die set for a specific design to punch large number of holes at the same time. The process is very fast but it is restricted to the specific design and the cost for modification or make any change in the design is enormous. Because of that, hard tooling is generally only used for high-volume production in well-established designs. In contrast, soft-tooling methods use a computer-controlled punch and die, which allows for rapid design changes that only require the change of a computer file. Computer programs are used to generate these files from CAD layout and to optimize the punching sequence for maximum speed. These soft-tooling processes are slower than hard ones but much more flexible in terms of their use for multiple designs or design revisions. In most cases, the tape layer is moved, and the punch and die are fixed with reference to one another. Accurately moving the tape layer and repeatedly moving the punch into the die at the desired location can fabricate a large number of vias very rapidly.

\subsection{Via fill}

To create electrical interconnects between layers, the via holes must be filled with conductive metal. This process is generally done by injecting a high viscosity thick-film ink into the holes with either stencil printing or a bladder-fill operation. The high viscosity of ink is designed to fill a 4 to $12 \mathrm{mil}(100-300 \mu \mathrm{m})$ via hole without running or sagging prior to drying. A solid plug remains inside of the via hole to entirely fill the hole after burning off the organics in the firing process. This plug creates a low-resistance electrical connection from one side of the tape to the other, as specified by the design. 

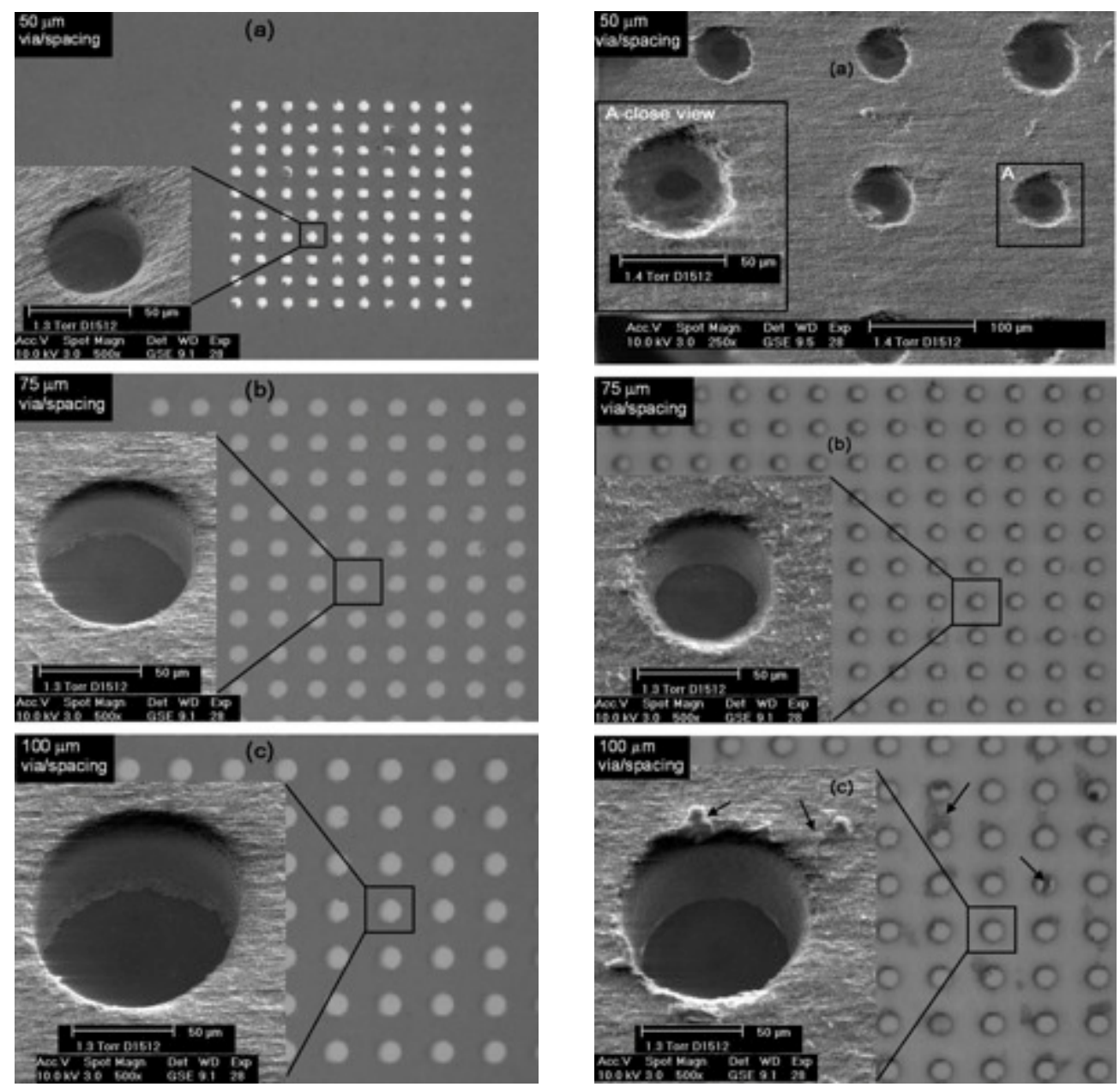

Figure 3-3Pictures of microvias in $163 \mu \mathrm{m}$ thick LTCC tape. Left) punched via. Right) laser-drilled via [109]

The stencil-fill process is very similar to conventional screen-printing; however, most foundries use a metal stencil rather than a mesh screen. The stencil is mounted in a screen printer that is usually configured in a way to be contact with the tape, which provides zero snap-off distance during the printing cycle. In this way, the printing process will not deform the stencil. The suitable viafill ink is applied to the stencil, and the printing process is then activated to fill the vias in a particular tape layer all in one pass. This process is repeated for each of the design layers in a given substrate to produce a sufficient quantity of each layer for the desired number of substrates. 
The bladder-fill process, similar to the stencil-fill process, uses stainless steel mask, however the injection processes are fundamentally different. A bladder filler uses a rubber bladder and compressed air instead of the shear force of the squeegee in stencil printing. Some tooling holes are also used in the stencil in addition to the via holes to align the stencil in the bladder-fill system. The paste to be injected is spread across the surface of the stencil, and then a clean rubber bladder is placed on top of the ink. This combination of stencil, ink, and bladder is loaded into the machine. A tape layer to be filled is placed on the stage of the machine, and then the machine is cycled to fill the tape layer. During this process cycle, the bottom of the stencil is brought into alignment with the tape layer using mechanical pins or machine vision to register the two components accurately. Pressure is then applied to the stencil or bladder assembly to maintain contact between the two and form an air-tight seal. Pressure is applied to the rubber bladder, which then forces the ink through the stencil and into the empty vias. This process only requires a few seconds, and the result is a tape layer with metal-filled vias [110]. Generally, a stencil will be loaded into a machine for a given design layer, and a large number of parts for the design layer will be filled in a consecutive manner. It is a common practice to use this method to fill thousands of vias in a given tape layer in one rapid process

\subsection{Tape layer collation and lamination}

To create the final substrate, the appropriate layers must be stacked in an intimate way so that they sinter together during firing and create final part. In this way, the layers should stack together first and then be laminated to create a solid block of ceramic and metal that contains the circuitry of interest. Two principal methods are used to achieve this alignment: optical alignments with machine vision and pin-alignment fixtures, called palters. 
The pin-alignment fixtures have four pins located at the corners of the tape blank, which are inserted from the edge of the tape layer. Holes are placed in a small distance and correspond to punched or cut holes in the tape layers. Each layer then identically placed over the pins and slipped down to the substrate. By locating each successive tape layer with respect to the pins and the identical punched holes, the tape layers can all be precisely aligned to the pins and therefore each other at the end. The principal limitation of this approach is that the tape layers tend to stretch and, in some cases, the holes in the tape are slightly deformed by the pins, which could create some alignment error. Optical alignment based on camera systems and computer-based machine vision is a far superior method that can minimize alignment errors. In a fully automated process, a machine picks up each layer by vacuum, and then a set of cameras will identify punched or printed structures on the tape layer. The identified identical alignments marks on each layer then are using to precisely align the layers to each other.

Once the layers have been collated or stacked, a more permanent bond is required prior to firing. High pressure and elevated temperature softens the polymer matrix of the green ceramic and facilitates good adhesion and coalescence of adjacent layers. This process helps the particles within adjacent layers to be in intimate contact during the firing process to prevent the formation of voids and delamination in the final substrate. The layers are very difficult to separate after lamination, and the substrate is ready for the firing and sintering process. Two principal methods are used for lamination: isostatic lamination and uniaxial lamination.

Uniaxial lamination is performed in a press that is equipped with heated plates. The part is often placed inside a metal fixture, which is usually the same one that is used in the collating step, and then placed in the laminator. The laminator applies heat and pressure to the fixture, transferring both to the ceramic tape layers. Fast and simple process operation is the most 
important advantage of the uniaxial press, however establishing perfect parallel lamination plates is difficult. Non-parallel plate could create non-uniform pressure across the plates, which cause non-uniformity across the laminated ceramic layers. Changing pressure, temperature and time may vary the green density of the ceramic and as a result the final density and shrinkage of the fired ceramic may be changed. In contrast, isostatic lamination uses water as a pressure media to apply uniform pressure and heat to the ceramic layers.

Because of the complex interaction of water and green ceramic, the stacked ceramic layers are vacuum bagged to prevent the introduction of water into the substrate prior to lamination. Metal plates may be used on either or both sides of the tape layers. At the bottom, a base plate is used to support the tape layers, while a cover plate on top may be used to distribute the pressure uniformly over the substrate. In this method, vacuum-bagged parts are often stacked inside the laminator on multiple shelves, and so many parts can be laminated in one lamination cycle with this approach at the same time.

\subsection{Firing and sintering condition}

Once the individual layers of green ceramic tape have been prepared and laminated together, the substrate must be fired to create the desired dense ceramic. This process can be performed as a batch process in a box furnace or in a continuous pusher kiln operation found in HTCC firing in a reduced atmosphere or belt furnace in air fired LTCC.. The advantages of utilizing a box furnace include improved control over the temperature uniformity and, in many cases, gas flow, as well as low price. Non-uniformity of temperature across the furnace during firing may cause the substrate to shrink in a nonuniform manner. In contrast, pusher kilns and belt furnaces offer much greater throughput because a continuous flow of substrates may be fired 
in this type of operation. Even though the firing process employed in the belt furnace is fundamentally different than the box furnace, the heating profiles may be very similar.

A common firing profile consists of heating and soaking in temperature below $450 \mathrm{C}$ to burn-out the organic. During this portion of the profile, the substrate evolves a large quantity of gas that must be removed from the furnace and vented. After this step the substrate is very fragile due to removing of the polymeric binders that rendered the material flexibility. In the second portion of the process, the materials in the substrate react, or sinter, to form the solid ceramic block that is the desired result. During this process, the remaining powders pack more closely together, resulting in shrinkage of the substrate in most material systems. This shrinkage generally occurs in all directions and ranges from 10 to $20 \%$, depending on the material and the direction.

The key for most applications is controlling or predicting this shrinkage so that the parts end up with the desired physical dimensions. The rate of densification and shrinkage depends on the particle size of the ceramic, firing atmosphere, heating rate and final firing temperature, which are discussed in detail. Finally, the substrate is cooled to room temperature. The proper furnace profile is important to achieve the desired final substrate density, as well as the desired mechanical and electrical properties

\subsubsection{Heating rate}

Understanding the grain growth and densification mechanism is critical to establish robust sintering conditions. Ever since the pioneer theoretical models $[111,112]$ were established, the sintering processes have been intensively studied for more than half a century. Evaluation of the activation energy of sintering is the key component to achieve this goal, and understanding the 
controlling mechanisms of the sintering process. Generally, two different types of methods are used for the evaluation of the activation energy:

- Isothermal methods, e.g. the rate controlled sintering (RCS) method

- Non-isothermal methods, e.g. the constant heating-rate method [113]

Even though, Young and Cutler [113] were the first who used the non isothermal method to calculate the activation energy, they concluded that this method could not be used to identify the mechanism of diffusion because of the wide variation of the evaluated activation energies. They pointed out that the particle size distribution might be the factor for the variation in evaluating the activation energy. In addition to that, different heating rate can change the sintering mechanism [114] as will be discussed later. Wang and Raj [115] developed a method for the evaluation of the activation energy based on an Arrhenius plot of $\ln (\dot{T} T)$ (where $\dot{T}$ is the densification rate, $\mathrm{T}$ the absolute temperature) versus the reciprocal of the absolute temperature at constant heating rates. The method that they developed was very easy and fast for calculation purposes.

$\ln (T \dot{T})=-\frac{Q}{R T}+\ln [f(\rho)]+\ln A-n \ln d$

where $\dot{T}(=d T / d t)$ is the heating rate, $R$ the gas constant, $\mathrm{f}(\rho)$ a function of density only, $A$ the material parameter which was insensitive to $d, T$, or $\rho, \mathrm{d}$ the particle size, $\mathrm{n}$ the particle size power law ( $\mathrm{n}=3$, if the densification rate was controlled by lattice diffusion, and $\mathrm{n}=4$ for grain boundary diffusion).

The isothermal and non-isothermal densification behavior, and their corresponding calculated Arrhenius plot of $\alpha-\mathrm{Al}_{2} \mathrm{O}_{3}$ classified powder with the average particle size of $0.24 \mu \mathrm{m}$ from the work of Wang and Raj [115] are shown in figure 3-4. Densification of alumina particle 
is almost $100 \%$ in both system, but their calculated activation energy exhibit a large difference. The calculated energy for isothermal densification is $452 \mathrm{kJmol}^{-1}$, while it is $739 \mathrm{kJmol}^{-1}$ for the non-isothermal method. The first one is close to the grain boundary diffusion of the aluminum ion of alumina and the later is close to the lattice diffusion of the aluminum ion of alumina.
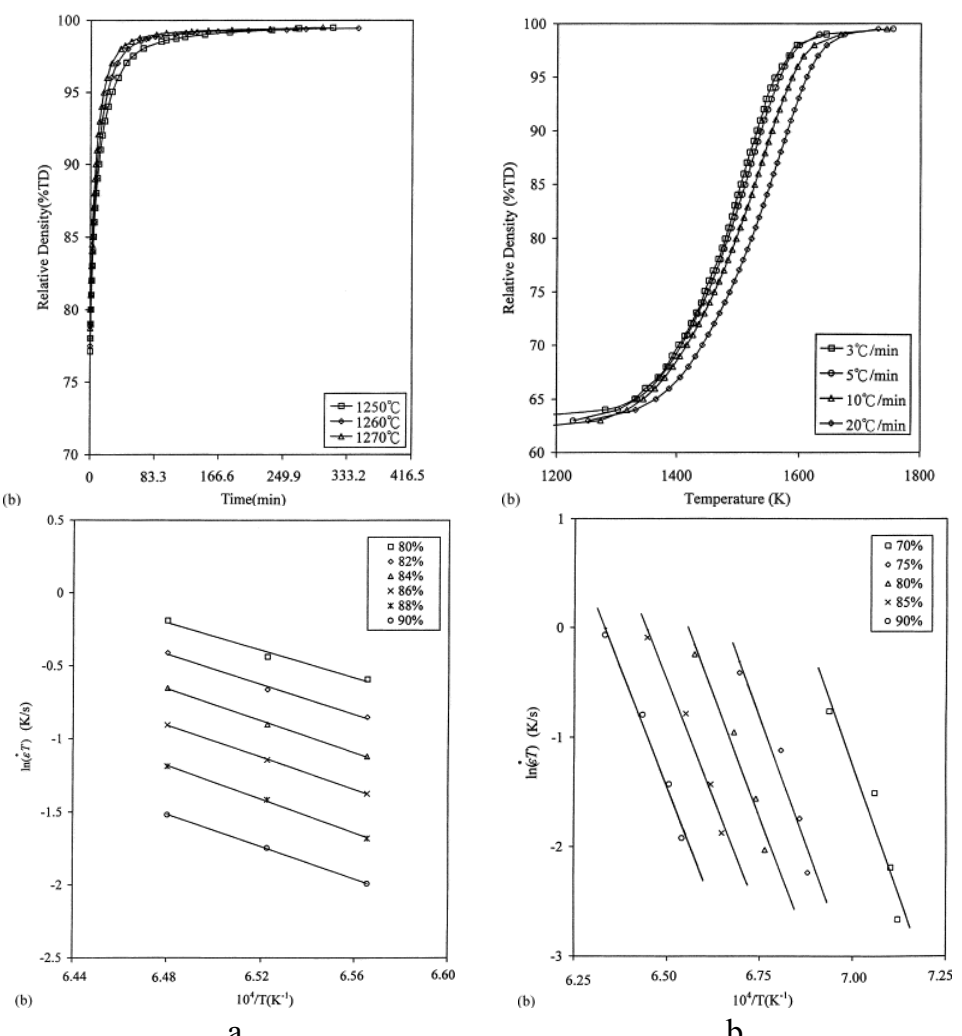

b

Figure 3-4The isothermal densification behavior and the Arrhenius plot of $\ln (\varepsilon T)$ versus (1/T). a) Isothermal sintering. b) non-isothermal sintering

According to the sintering theory, the activation energy of sintering should be consistent with the apparent diffusion coefficient of the rate-limiting species of sintering. In sintering polycrystalline ceramics, it is common that both grain boundary $\left(D_{b}\right)$ and lattice $\left(D_{l}\right)$ diffusion of the rate-limiting species can simultaneously contribute to densification. Because these two diffusivities have different temperature-dependence, the relative contribution of $D_{l}$ and $D_{b}$ is 
different at different temperatures. Thus, heating rate would have a significant influence on the non-isothermal kinetic processes due to the fact that it can change the relative contribution of $D_{l}$ and $D_{b}$ from low to high temperatures [116].

Fang et al. [114] showed that, the evaluation of activation energy depends on the heating rates, which are used in equation 36. They showed the values of activation energy based on different heating rates could be calculated, i.e., $478 \mathrm{~kJ} \mathrm{~mol}^{-1}$ for 10 and $20{ }^{\circ} \mathrm{C} \mathrm{min}^{-1}, 640 \mathrm{~kJ} \mathrm{~mol}{ }^{-1}$ for 5, 10, and $20 \mathrm{C} \mathrm{min}^{-1}$, and $1080 \mathrm{~kJ} \mathrm{~mol}^{-1}$ for 3,5 , and $10{ }^{\circ} \mathrm{C} \mathrm{min}^{-1}$, respectively. Shao et al. also showed that for the $\alpha$-alumina particle with the particle size of $2.5 \mu \mathrm{m}$, the calculated activation energy is, $1148 \mathrm{~kJ} \mathrm{~mol}^{-1}$ for 2 and $5 \mathrm{C} \mathrm{min}^{-1}$, and $1164 \mathrm{~kJ} \mathrm{~mol}{ }^{-1}$ for $0.5,2$, and $5{ }^{\circ} \mathrm{C}$ $\min ^{-1}$, respectively [117]. These values indicated that the higher value of the evaluated activation energy would be obtained when the Arrhenius plot involves low heating rates. For isothermal sintering, because the difference among the sintering temperatures is small, the influence of the relative contribution of $D_{1}$ and $D_{\mathrm{b}}$ to the densification will also be small at the same density level, however for non isothermal densification, the relative contribution of $D_{1}$ and $D_{\mathrm{b}}$ to densification is different at the same density level for the high and low heating rates.

It is accepted that the grain boundary diffusion (GBD) or volume diffusion (VD) are two prominent mechanisms for densification in porous ceramic compacts. It is noted that the dominant path for diffusion is usually along the grain boundary. Classical grain-boundary diffusion mechanism assumes that grain boundaries act as perfect sources and sinks for the diffusing atoms during the diffusion process and the energy provided is all available to drive the diffusional flux along the grain boundaries. The low GBD activation energy (compared with VD) is because the grain boundary is more active and it serves as a tunnel for diffusion. 
During densification, the displacements of alumina and oxygen vacancies are both of great importance. In that case, it is thought that the role devoted to Schottky disorders (equilibrium constant is $K_{S}=\left[V_{A l}^{\prime \prime \prime}\right]^{2}\left[V_{O}^{\infty}\right]^{3}$ using the Kröger-Vink formalism) is critical. El-Aiat [118] has also shown that the Schottky disorder energy of formation in alumina is roughly $630 \mathrm{kJmol}^{-1}$ [31].

It has also been shown that the activation energy for the grain boundary diffusion of $\mathrm{Al}^{3+}$ in alumina is $420 \mathrm{kJmol}^{-1}$ [33]. In this way, Bernard-Granger et al. [119] proposed that densification is controlled by the formation of Schottky disorders and by the diffusion of the atoms associated to the Schottky disorders, the grain boundary diffusion of the $\mathrm{Al}^{3+}$ cations being the limiting step. The total apparent activation of such a mechanism is $1,050 \mathrm{kJmol}-1(630+420$ $\mathrm{kJmol}^{-1}$ ), which is close to their calculated activation energy $1,095 \mathrm{kJmol}^{-1}$ for heating rate of 1.6 ${ }^{\circ} \mathrm{C} \min ^{-1}$. However based on this formulation, the densification through volume diffusion has an activation energy around $1300 \mathrm{kJmol}^{-1}$, which could only be achieved through very low heating rate.

\subsubsection{Particle size and particle size distribution}

Particle size and particle size distribution are one of the critical parameters that could control the sintering mechanism and control the sintering kinetic. Yeh and Sacks [120] used powders with known particle size but two different distribution and showed thatboth powders gave comparable end density and resultant grain size on further sintering. However, the powder with broader distribution gave a higher green density and improved densification behavior during the initial stage of sintering, but its densification rate decreased faster on continued sintering. They therefore concluded that there is no advantage in using the powders with narrow size distributions for it has a lower starting green density, hence a larger overall shrinkage. In general, 
a broader particle size distribution is expected to lead to a higher green density due to the gap filling ability of the fine particles. A broader particle size distribution also promotes grain growth, which in turn would modify the evolution of pores, hence the densification behavior of the compact. On the other hand, too broad a particle size distribution may cause the sintering characteristics of the compact to deteriorate due to the low-sintering characteristic of the much coarser particles. Ma and Lim [121] showed that the optimum particle size distribution may vary depending on the consolidation technique and conditions used. They suggested that narrow size distribution powder is preferred to monosized or broad size distribution powders for improved sinterability and high-reliability microstructure control of powder compacts.

Comparing the "relative grain size/relative density" trajectory, called the "sintering path", of particle with different particle size is shown in figure 3-5, Lim et al. [122] showed that grain growth is necessary for further densification at the final stage of sintering. Densification and growth increase by decreasing particle size. Difference in the densification behavior of the particle with different particle size could be due to their sintering mechanism. He and Ma [123] compared two particles with the particle size of $7 \mu \mathrm{m}$ and $0.9 \mu \mathrm{m}$ and showed that grain boundary diffusion is the rate controlling step and the appropriate mechanism for large particle. For smaller particles, grain boundary diffusion is the fastest process due to the shorter transport path and hence the interface reaction becomes the controlling mechanism. On the other hand, Lim et al. [122] showed that for $\alpha-\mathrm{Al}_{2} \mathrm{O}_{3}$ with the average particle size of $0.18 \mu \mathrm{m}$, the grain boundary diffusion is the more appropriate mechanism than the lattice boundary diffusion. Shao et al. [117] calculated the densification activation energy for the powders with two particle size using the master sintering curve technique. They showed that the powder with particle size distribution of $2-3$ and $0.2-0.5$ has the activation energy of $1148 \mathrm{kJmol}^{-1}$ and $1035 \mathrm{kJmol}^{-1}$ respectively. 
They also suggest different densification mechanism for different particle, however the exact detail was not described.

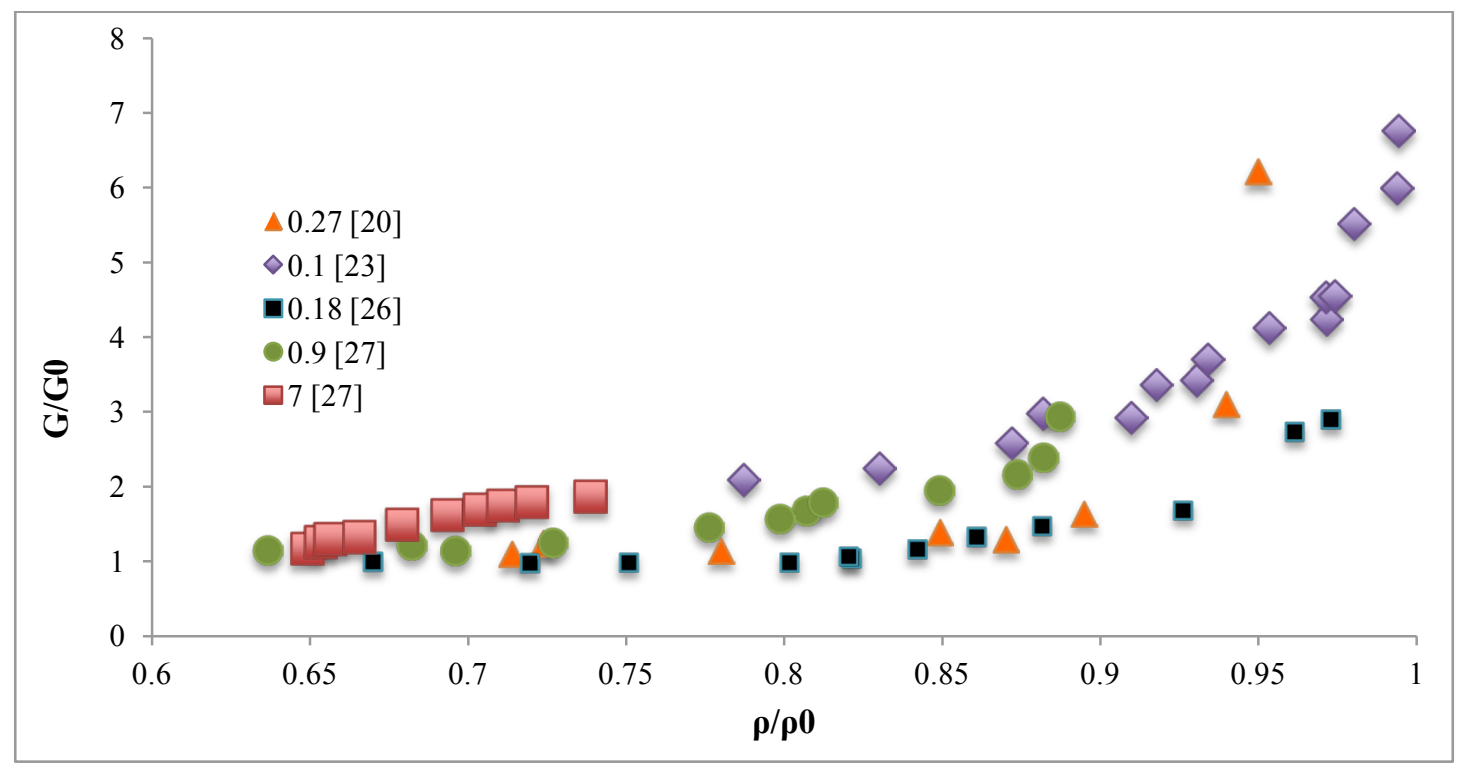

Figure 3-5 Variation of the relative grain size to the relative density of the particles "Sintering path" of alumina particle with different particle size. Experimental data are from Refs. [115, 119, 123, 124]

One of the ways to decrease the grain size and reduce the sintering temperature is mechanical milling [124]. High energy mechanical milling has been used to produce nanometer sized particles in many materials including alumina [125]. However for an extremely hard material like alumina, milling media can create the contamination. Forrester et al. [126] used ball-to-powder ratio of 3:1 and increased the milling time to decrease the contamination. They showed that the alumina crystallite size has reduced to approximately $29 \mathrm{~nm}$ after 64 hours of milling, and in that case some sintering occurs as low as $400{ }^{\circ} \mathrm{C}$.

\subsubsection{Sintering atmosphere}

Sintering atmosphere has two major important effects on the sintering process. Changes of atmosphere may change the densification rate or the limiting final density. For oxides, the 
dependence of sintering rate on atmosphere is presumed to be due to a change in the number of vacancies with changing oxygen pressure in the atmosphere, and the number of vacancies could control the diffusion rate. It is assumed that lattice diffusion $\left(D_{l}\right)$ takes place by the reverse flux of lattice vacancies $\left(D_{v}\right)$. Onsager showed $D_{v} n=D_{l} N$, in which $n$ is the number of vacant lattice sites per unit volume and $N$ is the number of lattice sites per unit volume. The direct effect of atmosphere on the number of one kind of vacancies also affects the number of the other species because of the equilibrium between the Schottky vacancy pairs. For diffusion sintering an increase in the number of lattice vacancies of the slower diffusing species increases the lattice diffusion coefficient of that species and therefore the sintering rate.

However, during the shrinkage process, the open pore channels are pinched off at the specimen surface first (because of faster sintering on the pores) and the trapped gas can no longer escape to the atmosphere. Vines et al. [127] demonstrated that in the final step of processing porcelain glass-ceramics, gas diffusion controls the shrinkage rate, indicating that in the lowest gas diffusivity, pores shrinks to their stable sizes. Increasing the diffusivity of the gas increases the shrinkage of the pores and shrinkage rate starts to be controlled by the kinetic of the gas diffusion to the surface. At quite high diffusivity of the gas, the final shrinkage is physically independent of the gas.

Coble [128] studied the sintering of alumina with $0.25 \%$ (wt) $\mathrm{MgO}$ in different atmospheres. He showed that, among all the gases that he used, hydrogen and helium presumably could diffuse interstitially. The difference in sintering behavior between them is attributed to the differences in their solubility in alumina. Hydrogen can react with alumina, so it is assumed that its solubility is greater than the noble gases. In addition to that, hydrogen could reduce the spinel phase content formed by the reaction of the $\mathrm{MgO}$, and increased magnesia 
distillation in a hydrogen atmosphere. Coble showed that the sintering of alumina in hydrogen could get to the density of almost $100 \%$.

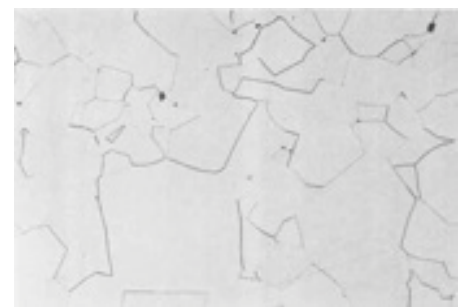

(a)

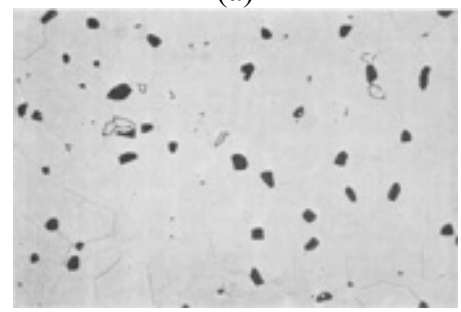

(d)

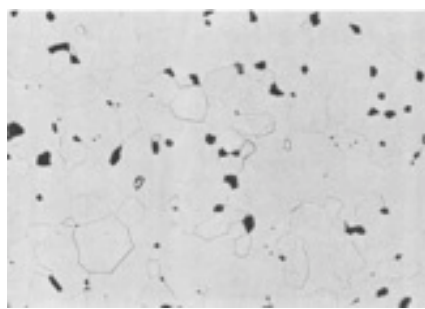

(b)

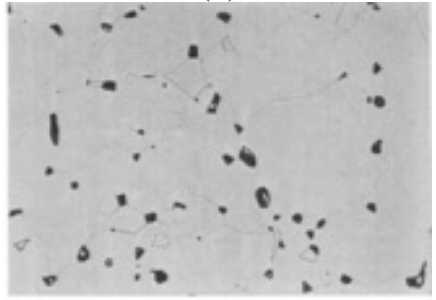

(f)

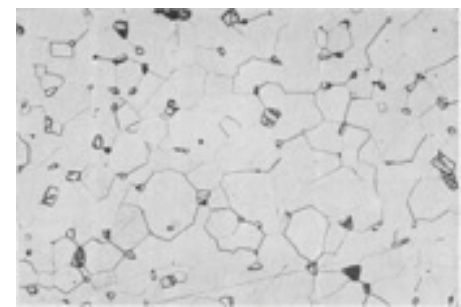

(c)

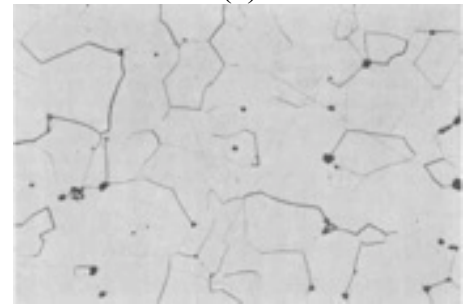

(g)

Figure 3-6 Sintering behavior of alumina in different atmosphere. a) Hydrogen, b) Helium, c) Oxygen, d) Nitrogen, f) Argon, g) 7\% Nitrogen+93\% Oxygen

Comparison of oxygen, air, nitrogen and argon showed that, alumina samples fired in oxygen with $7 \%$ nitrogen atmosphere sinter to its theoretical density. Sintering in air showed that there are many pores (black areas) remaining on which the grain boundaries tend to be pinned, and these pores limited the density of the sample to $97 \%$. The rate of sintering was slower in air because of an effect on the stoichiometry, the excess oxygen or nitrogen, or both, diffused more slowly in the alumina lattice or in the grain boundaries than did the stoichiometric lattice species. Oxygen may enter the solution by changing the stoichiometry as the gas pressure in the pores increases. The magnitude of such change is not known for the intrinsic behavior in alumina. Argon and nitrogen are too large to enter interstitial positions and are expected to displace oxygen ions in order to enter solution. Diffusion of these solutes then depends on the diffusion of the neighboring oxygen ions, and they should therefore exhibit kinetics equivalent to oxygen. A difference between excess oxygen and nitrogen or argon in solution is that the excess oxygen diffuses (at least) as rapidly as oxygen vacancies. The actual equilibration of the excess oxygen 
may be even faster since it is not confined to diffusion of oxygen lattice sites or vacancies at all but could occur by the diffusion of aluminum vacancies from the outer surface of the specimen to the pores. Thus, for oxygen, equilibration can occur by diffusion of the faster moving species, whereas for argon or nitrogen, movement is probably limited by oxygen diffusion.

In contrast to the sintering theory of oxides, information available about the detailed sintering kinetics of metals are incomplete. Hassan [129] studied the sintering and densification of the platinum black in oxygen, hydrogen and nitrogen atmosphere up to $600{ }^{\circ} \mathrm{C}$. Densification of the metal in catalysts corresponds directly to its loss of catalytic activity. He showed that that the rate of sintering of platinum differs widely in various atmospheres, as shown in figure 3-7.

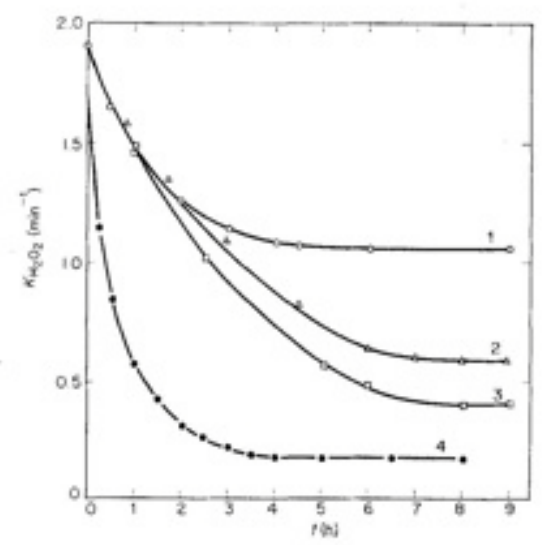

Figure 3-7 The change of catalytic activity of Pt-black catalyst and correspondingly it's densification as a function of time of sintering a $300 \mathrm{C}$ in (1) oxygen atmosphere, (2) nitrogen atmosphere, (3) vacuum and (4) hydrogen atmosphere [129].

The highest catalytic deactivation and densification was achieved in platinum and lowest happened in oxygen. The sintering goes much more easily in hydrogen rather than in other atmospheres due to the reduction of $\mathrm{PtO}_{2}$ layer [70] by hydrogen. Glassl et al. [130] showed that the platinum is densifed up to $45 \%$ of its original structure in hydrogen in $510^{\circ} \mathrm{K}$ and decrease further up to $25 \%$ in $720^{\circ} \mathrm{K}$. He also did the same experiments for oxygen and argon and 
concluded that the rate of densification in oxygen is less than argon and in argon is less than in hydrogen.

The activation energy of the sintering process was found by Hassan [129] to be $12.5,14.6$ and $52.3 \mathrm{kJmol}^{-1}$ in hydrogen, oxygen and nitrogen atmospheres respectively as obtained from the surface measurements, assuming the Arrhenius equation is valid. Higher sintering activation energy for nitrogen suggests a different mechanism than in oxygen and in hydrogen. Nitrogen might combine with the chemisorbed oxygen on the surface to form $\mathrm{NO}_{2}(\mathrm{~g})$ that is stable up to $2000^{\circ} \mathrm{K}$. The heat of formation of $\mathrm{NO}_{2}(\mathrm{~g})$ is approximately $32.4 \mathrm{kJmol}^{-1}$ and if the desorption of NO, on the surface becomes the rate determining step, this minimum value should be supplied as an energy of activation. This could be the interpretation of the high activation energy. From these results, it is expected that the activation energy of sintering of platinum in argon is somewhere between hydrogen and oxygen

Gland et al. [70] showed three distinct types of interactions of oxygen with platinum surface. In general, molecular oxygen predominates following adsorption below $120^{\circ} \mathrm{K}$. Analysis of thermal desorption of atomic oxygen in the literature indicate a heat of adsorption of about $37 \mathrm{kJmol}^{-1}$. Heating adsorbed molecular oxygen layer above $170{ }^{\circ} \mathrm{K}$ results in the formation of adsorbed atomic oxygen. This atomic adsorption predominates between 170 and about $700{ }^{\circ} \mathrm{K}$. Platinum surfaces can be oxidized by high temperature oxygen treatments in the temperature range of 800 to $1100{ }^{\circ} \mathrm{K}$. In this high temperature oxide, oxygen has moved below the platinum surface, thus the oxide is non-reactive below $1100^{\circ} \mathrm{K}$, compared to adsorbed oxygen atoms.

Reaction of hydrogen with platinum in different temperatures could reduce each of these oxygen layers and enhance the densification. Above temperature of $1100^{\circ} \mathrm{K}$, the oxide films are 
no longer stable any longer and the metal surface remains clean because of the high dissociation pressure of the oxides. At the temperatures that high, however, volatile oxides are formed. Even if the evaporation rate of the oxides is relatively low, the metal losses in oxygen-containing atmospheres can be much higher than those obtained when the pure metals are heated in high vacuum or in inert atmospheres [131-133]. This suggests that the shrinkage of the platinum in air or in oxygen atmosphere at high temperature is more sever than hydrogen.

\subsection{Design consideration}

The intrinsic multilayer approach of cofired ceramic offers many advantages to designers. However some consideration also should be put in place to develop a proper product. Shrinkage of the cofired ceramic due to the sintering process is occasionally cited as a problem with this technology. The key is to understand and control the process and the material variables that lead to uncertainty in the shrinkage [97]. Mismatched sintering kinetics between the metal and the ceramic can lead to significant undesirable defects like delamination, cracks, camber or warpage in the product, which substantially lowers production yield[134]. The mismatched sintering kinetics of the two materials lead to the development of in-plane stresses in the two constituents of the composite structure as one material being constrained from sintering by the other. The resulting defects during the co-firing process could be explained by the development of these stresses.

Difference in thermal expansion, densification rate mismatch, or combinations of them between the layers could be assumed to be the stress generator. Thermal expansion mismatch is more likely to produce defects in the layer with a larger thermal expansion coefficient during cooling since this layer will be subject to tensile stress. Cofired defects are more frequently 
generated in the layer, which densifies more rapidly, resulting in a biaxial tension during sintering. A crack with a large opening and a blunt cavity-like appearance is generally believed to be caused by the densification rate misfit, but a crack with a small opening or displacement may be the result of thermal expansion mismatch and brittle in nature [135].

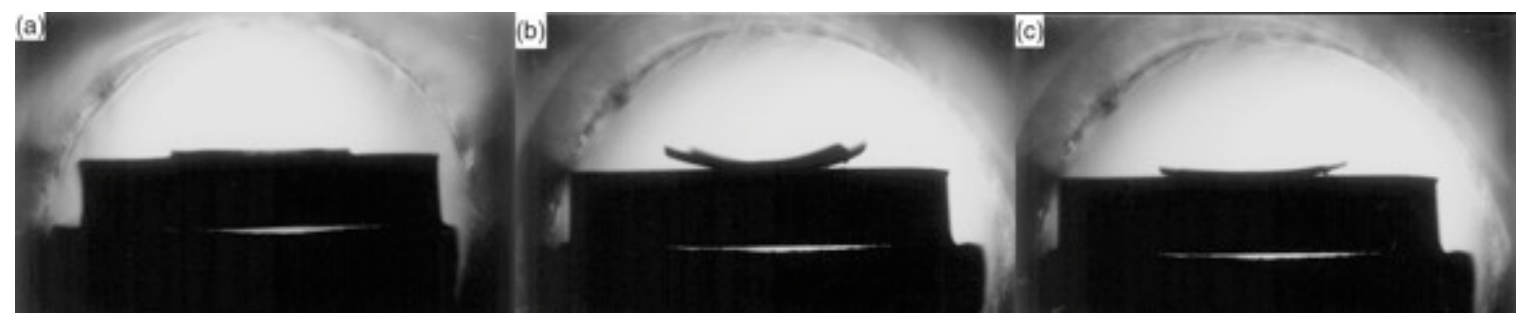

Figure 3-8 Photos of camber development for the Ag/LTCC (A) room temperature, (B) $750{ }^{\circ} \mathrm{C},(\mathrm{C}) 850{ }^{\circ} \mathrm{C}[136]$

To minimize the possibility of forming the cofiring defects, a more thorough understanding of the process resulting in the above defects is needed before the processing parameters and material compositions can be optimized. Typical solutions for minimizing camber have often relied upon engineering approaches, such as weighting, varying material compositions, and varying the co-firing temperature-time profiles. For example, balancing printed metal and via densities throughout a design can significantly reduce the potential for camber or a lack of uniformity in the shrinkage because the metal density affects the shrinkage of many of the material systems.

However, more basic understanding of the processes that result in camber could be useful to predict and prevent such defects. Lu et al. [134] first proposed a model based on viscous constitutive relations for porous sintering bodies to model the developed stress during the sintering process. Jean and Chang [135-138] in a series of publication studied the chamber development in the LTCC with different conductive ink, and model it on the basis of elastic and viscous constitutive relations analogous to the thermal stress problems arisen from the difference in thermal expansion coefficient in a thin plate. They showed that the ink has a lower onset 
densification temperature, but a faster densification rate and a greater total shrinkage, compared to the ceramic tape. This different densification indicates different sintering mechanisms. They suggested that grain boundary diffusion and viscous flow of glass are the sintering mechanisms for ink and tape respectively. Their results suggested that viscous constitutive model has a good agreement with experimental observations $[135,136]$. 


\section{Experimental Procedures}

\subsection{Materials}

\subsubsection{Metal powder}

Seven different platinum powders were used in this study. Five powders used to make ink and produce the final feedthrough assembly and the other two were used for high temperature behavior study. Platinum powders, primarily from Heraeus Thick Film Materials, Conshohocken, PA and Alfa Asar were used. The complete list of their properties and specification are shown in table 4-1. The Pt-3 particle was provided form the Heraeus Co. in ink form, so the exact properties of the particle are not able to be fully characterized.

Tungsten, iridium and rhodium were used as additives to the platinum ink. Two tungsten particles with different particle size provided to study the effect of particle size on the sintering behavior. All metal particles were purchased from Alfa Asar Co.

\subsubsection{Oxides and glass particles}

Aluminum oxide $\left(\mathrm{Al}_{2} \mathrm{O}_{3}\right)$, yttrium oxide $\left(\mathrm{Y}_{2} \mathrm{O}_{3}\right)$ and tungsten oxide $\left(\mathrm{WO}_{3}\right)$ were used in this project as the shrinkage-controlling agent in the conductive ink. Nagai et al. [139] showed that oxide with $\mathrm{O}(1 \mathrm{~s})$ binding energy less than $530 \mathrm{ev}$ could react with platinum and inhibit its sintering. In this way $\mathrm{Al}_{2} \mathrm{O}_{3}$ is non reactive, $\mathrm{Y}_{2} \mathrm{O}_{3}$ is reactive and $\mathrm{WO}_{3}$ is in the middle. Alumina is commonly use additive for conductive ink to control the shrinkage, because of its low thermal expansion and its compatibility with the substrate. Yttrium oxide was chosen to inhibit the sintering of platinum. Powel [140] showed that yttrium oxide can react with the platinum and decrease the densification rate. Tungsten oxide could decompose to tungsten and oxygen at high 
temperature and the resulting tungsten particle could prevent the sintering of platinum at high temperature. Oxides are typically used with a $20 \%$ volume concentration in the ink formulation.

In addition to oxides, kaolinite clay and talc, a common glass forming formulation, were also used in ink fabrication to increase the metal/ceramic interface adhesion and reduced the sintering temperature. The properties of all oxides particles are also shown in table 4-1.

Table 4-1 The properties of the particles used in this project

\begin{tabular}{|c|c|c|c|c|c|c|}
\hline Code & $\begin{array}{l}\text { Chemical } \\
\text { Compound }\end{array}$ & Commercial name & Provider & $\begin{array}{c}\text { Surface } \\
\text { area }\left(\mathrm{m}^{2} / \mathrm{g}\right)\end{array}$ & $\begin{array}{c}\text { Tap density } \\
\left(\mathrm{g} / \mathrm{cm}^{3}\right)\end{array}$ & Particle size \\
\hline Pt-1 & Platinum & $\begin{array}{l}12076 \text { Platinum } \\
\text { black }\end{array}$ & Alfa Aesar & 10 & - & - \\
\hline $\mathrm{Pt}-2$ & Platinum & $\begin{array}{l}12755 \text { Platinum } \\
\text { black }\end{array}$ & Alfa Aesar & 27 & - & - \\
\hline Pt-3 & Platinum & CL11-5100 & Heraeus & - & - & - \\
\hline Pt-4 & Platinum & Pt-PM100-10 & Heraeus & $10.0-18.0$ & $0.6-1.8$ & - \\
\hline Pt-5 & Platinum & 44010 & Alfa Aesar & 1.5 & $2.5-6.0$ & $\begin{array}{c}90 \% \leq 1.5 \\
\mu \mathrm{m}\end{array}$ \\
\hline Pt-6 & Platinum & Pt-PM100-3 & Heraeus & $0.5-3.0$ & $2.0-3.5$ & $3.0-7.0 \mu \mathrm{m}$ \\
\hline Pt-7 & Platinum & M579B & Heraeus & $0.8-2.0$ & $3.0-4.0$ & $\begin{array}{c}0.9-1.9 \\
\mu \mathrm{m}\end{array}$ \\
\hline Ir & Iridium & 43783 & Alfa Aesar & $30-40$ & - & - \\
\hline $\mathrm{Rh}$ & Rhodium & 12353 & Alfa Aesar & - & - & - \\
\hline W-1 & Tungsten & 10400 & Alfa Aesar & - & - & $1-5 \mu \mathrm{m}$ \\
\hline $\mathrm{W}-2$ & Tungsten & 44210 & Alfa Aesar & - & - & $<1 \mu \mathrm{m}$ \\
\hline n-A12O3 & $\mathrm{Al}_{2} \mathrm{O}_{3}$ & MKN-Al2O3-A040 & mnNANO & - & - & $40 \mathrm{~nm}$ \\
\hline $\mathrm{Y}_{2} \mathrm{O}_{3}$ & $\mathrm{Y}_{2} \mathrm{O}_{3}$ & Yttrium (III) oxide & Sigma-Aldrich & 5.01 & - & - \\
\hline $\mathrm{WO}_{3}$ & $\mathrm{WO}_{3}$ & $\mathrm{WO}_{3}$ Nanopowder & Sigma-Aldrich & 7.5 & - & $<100 \mathrm{~nm}$ \\
\hline
\end{tabular}

\subsubsection{Ceramic tape}

Two different HTCC ceramic tapes were used to develop the final feedthrough assembly. The standard 96\% alumina tape from Maryland tape casting Co. and 92\% HTCC tape from Adtech Co., were primarily used for the project. 


\subsubsection{Polymeric binder}

The thick film via binder formulation was provided from Heraeus Co.. The binder is a proprietary an acrylic polymer with additives to enhance the wettability and rheology of the via filling ink. The binder components were weighted and mixed in the closed container. The container was heated to $80{ }^{\circ} \mathrm{C}$ for $10 \mathrm{~min}$ to decrease the binder viscosity for better mixing and then rolled for $20 \mathrm{~min}$ to achieve the desired level of homogeneity.

\subsection{Platinum/alumina sample preparation}

\subsubsection{Powdered sample}

A 1/4" die pressed with 300 Psi pressure was used to palletize the platinum and tungsten powders. However high pressure in the metal powder makes them to act like the bulk material and less like powder. Therefore, $1 / 8$ " palate die with lower pressure were use for metal powders for the rest of the experiments..

Pt-4 and nano alumina particles were used to study the metal/ceramic intermetallic reaction. The different ratios of Pt/Alumina were prepared based on the known intermetallic compounds of the $\mathrm{Pt} / \mathrm{Al}$ phase diagram. The compounds were then ball milled for an hour to reach an acceptable mixing. Additional mixing was prevented to minimize the degree of contamination of milling media into the samples. The mixed sample were then palletize with the same way metal powders were fabricated. 


\subsubsection{Platinum thin and thick film}

Two series of platinum film with the thickness of 50 and $200 \mathrm{~nm}$ were deposited on the two different alumina substrate at EIC Laboratories Co. The standard CoorsTek Thin-Film Superstrate 99.6 Alumina Substrates and single crystal sapphire were used to study the behavior of platinum thin film on alumina in high temperature. A Pt-4 thick film with $10 \mu \mathrm{m}$ thickness was applied on the same substrates to study the effect of thick films at high temperatures.

\subsection{Feedthrough development}

\subsubsection{Ink development}

Inorganic powders and organic binders were weighted and are hand mixed on the glass plate until thoroughly wet slurry is obtained. The prepared slurry was then mixed for 5 minutes on a Hoover Color Muller parallel plate mixer to achieve the best dispersion.

\subsubsection{Feedthrough assembly}

The conventional multilayer ceramic production process, as described completely in chapter 3, was used to develop the final feedthrough assembly. HTCC ceramic alumina tape first laser cut to the 160 by $160 \mathrm{~mm}$ sheet. The ceramic tape was then punched with a Keko PAM mechanical punch machine. 100,150 and $250 \mu \mathrm{m}$ size diameter punchs were used for production of different samples. Punched vias were filled with conductive ink by the means of a PTC-1000 vacuum assisted bladder filler. Clapping time, injection time and injection pressure are controlling parameter in the filling process. Different injection time and pressure were examined during the project to achieve the optimum filling condition. The optimum filling condition, 10 
sec clamping time, $20 \mathrm{sec}$ injection time and 15 psi injection pressure was used to fill the via in the entire project. A stereomicroscope with $30 \mathrm{X}$ magnification was used to check the alignment of the mask and ceramic tape in the filling process. The image of punched tape and tape filled with conductive ink are shown in figure 4-1.

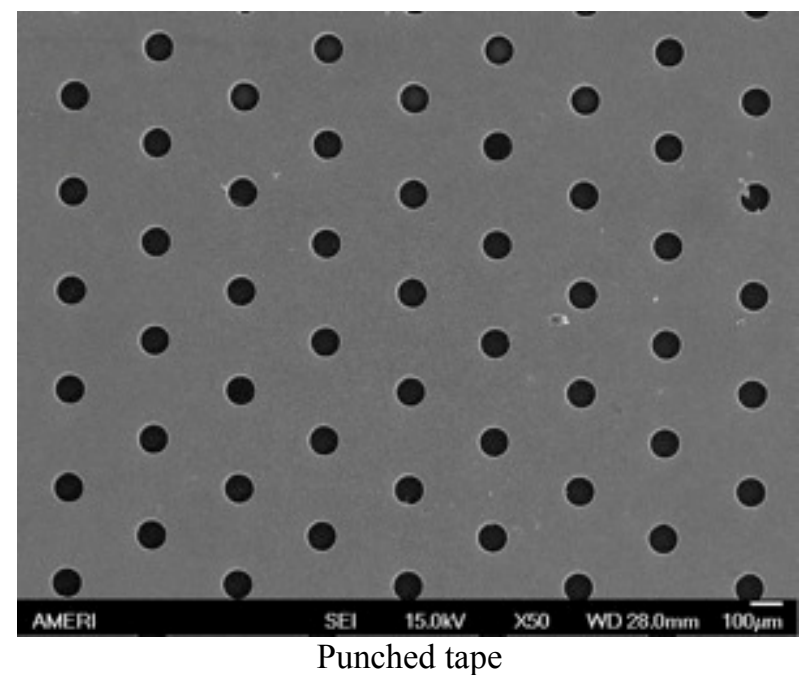

Figure 4-1 Punched and filled tape

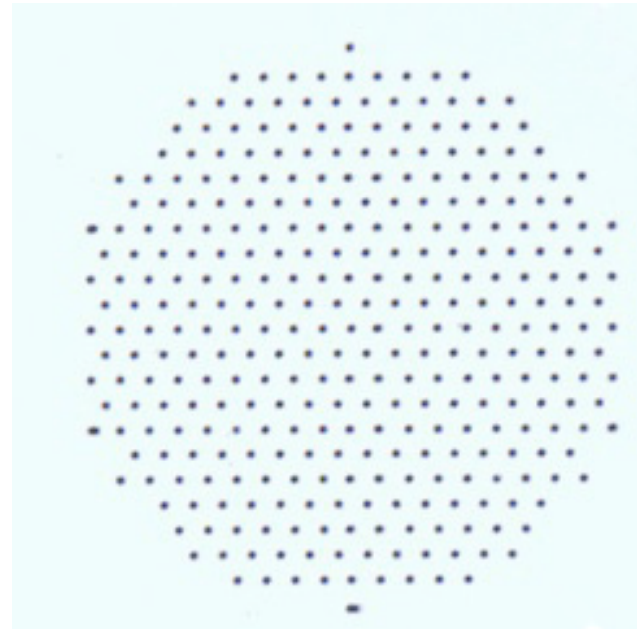

Tape filled with ink

Filled tapes were cut to the 1" square shaped and a pin-alignment fixture was used to manually collate the sample. It was then isostatically laminated in the PTC isostatic lamination machine under $3000 \mathrm{psi}$ and $70^{\circ} \mathrm{C}$. The sample was then cut to size with an OZO CNC milling machine and fired up to the temperatures range of $1050-1550{ }^{\circ} \mathrm{C}$ in different atmosphere and with different heating rate. The picture of complete feedthrough assembly is shown in figure 4-2.

A Lindberg 1700 muffle furnace and GSL-1600 X tube furnace were used for firing in air and the G-1600 hydrogen furnace from Camco Furnace Co. was used to fire the sample in reduced atmosphere. Different mixtures of $\mathrm{H}_{2} / \mathrm{Ar}$ were used to study the effect of hydrogen concentration on the final product. The furnace was equipped with the water bubbler to provide the wet hydrogen (variable partial pressure of oxygen) atmosphere to provide oxygen for the burn-out of the organic. A small self-fabricated hydrogen furnace with tungsten wire heater also 
used for some experiments, as shown in figure 4-3. All furnaces are equipped with the digital thermo-controller to program the firing conditions.

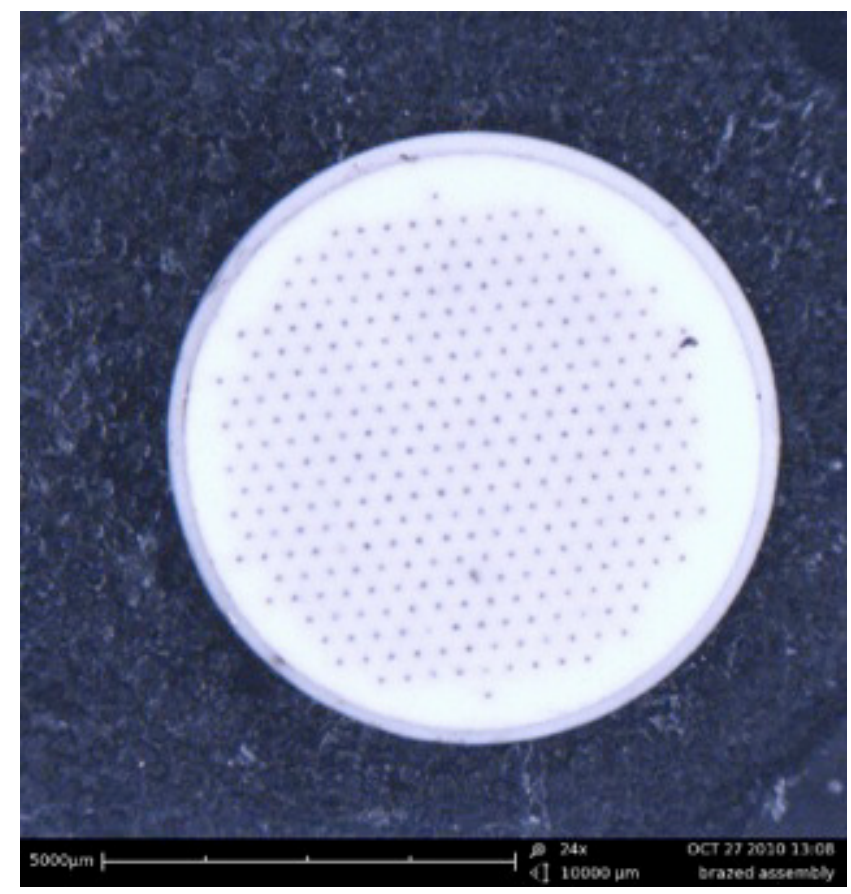

Figure 4-2 Complete feedthrough assembly
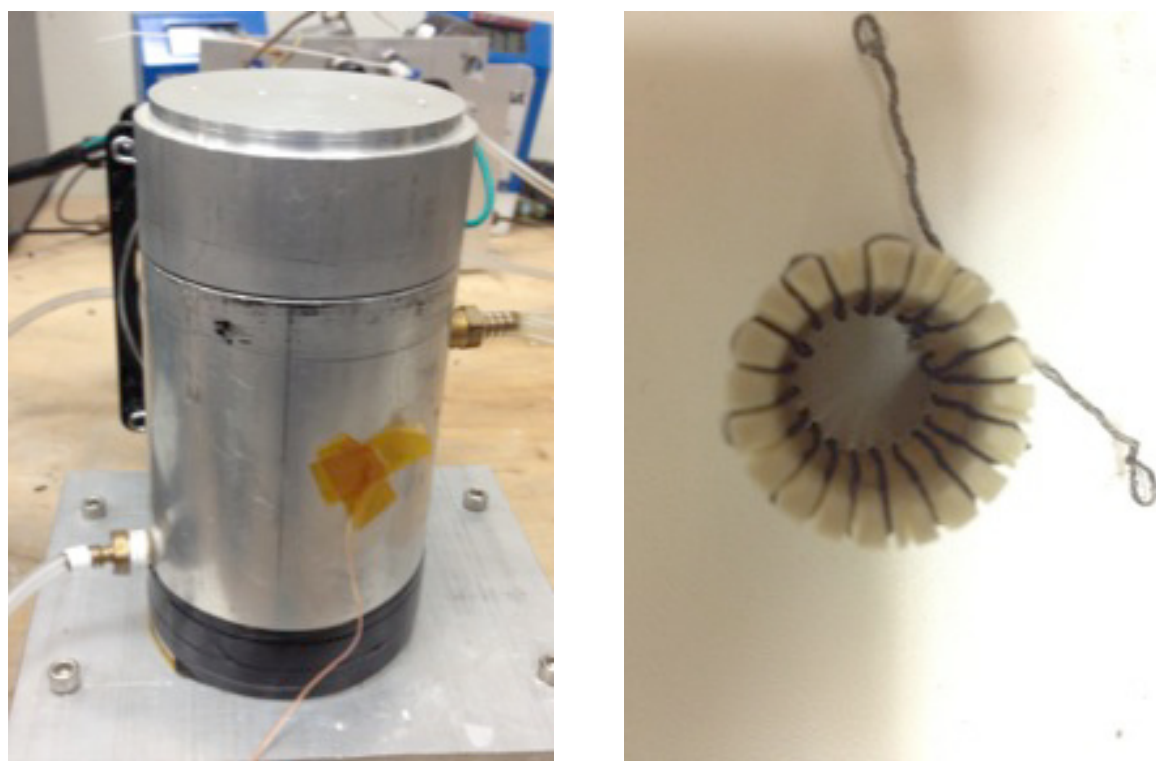

Figure 4-3 The structure of the small self-fabricated hydrogen furnace 


\subsection{Characterization techniques}

\subsubsection{Electron microscopy}

A JEOL JSM-6330F field emission scanning electron microscope (FE-SEM) with a magnification range of $150 \mathrm{KX}$ operating at $15 \mathrm{KV}$ and a JEOL $7000 \mathrm{~F}$ hot filament field emission scanning electron microscope, with a magnification rage to $300 \mathrm{KX}$, were used for the characterization of powders, sintered pellets, thin films and final feedthrough assembly. The low magnification $(20 \mathrm{KX})$ backscatter electron PHENOM tabletop SEM was used to take a backscatter SEM image of the samples. The Tabletop SEM is a low voltage and high pressure SEM from FEI. This SEM uses a $\mathrm{CeB}_{6}$ source, permanent magnets for electron focusing, and backscatter detectors arranged in four quadrants. This microscope is operated with a $5 \mathrm{KV}$ acceleration voltage and a chamber pressure of $\sim 0.3 \times 10^{-3}$ mbar. A dedicated quadrant backscattered detector is used for imaging. The PHENOM Desktop SEM shows advantages for imaging non-conductive samples in the low magnification range, which provides a sharp image with high depth of focus, large view area, and less damage of the sample.

Non-conductive samples were sputter coated with gold before observing in SEM. Cross section samples were cut with a slow speed diamond saw with diamond blade, mounted on a tripod sample holder, and metallographically grinded and polished for microstructural characterization. A tripod polisher was utilized to insure accurate polishing of the sample in the desired $\mathrm{x}, \mathrm{y}$ and $\mathrm{z}$ direction.

\subsubsection{X-ray diffraction measurement}

Powder X-ray diffraction data was obtained with a Siemens X-ray system consisting of a direct drive rotating anode as the $\mathrm{x}$-ray generator $(18 \mathrm{~kW})$. MoKa radiation (tube voltage $50 \mathrm{kV}$, tube current $24 \mathrm{~mA}$, cathode gun $0.131 \mathrm{~mm}$ ) monochromated using an incident beam graphite 
monochromator was passed through a collimator of diameter $200 \mu \mathrm{m}$ to the sample with the wavelength of $0.71073 \mathrm{~nm}$. The diffracted $\mathrm{x}$-ray was collected on a $512 \times 512$ pixels imaging plate area. Data were acquired for $2 \theta$ range of 5 and $40^{\circ}$. Settings of the detector were carefully calibrated using the $\mathrm{CeO}_{2}$. Due to small size of the samples, the time of data collection was 30 minutes, about 50\% longer than a standard sample.

\subsubsection{High temperature in-situ X-ray diffraction measurement}

In an attempt to better understanding the reaction between platinum and alumina, high temperature $\mathrm{Pt} / \mathrm{Al}_{2} \mathrm{O}_{3}$ reactions were studied using in-situ X-ray diffraction. The sample was resistively heated in a specially designed high temperature cell, as shown in figure 4-4. The description of the high temperature cell can be found elsewhere [141]. The sample was loaded and heated in a $0.15 \mathrm{~mm}$ hole which was drilled though the $1 \mathrm{~mm}$ thick graphite heating element. The temperature of the sample was measured using a $d$-type thermocouple and by measuring the thermal expansion of platinum [142] by in-situ x-ray diffraction from (111), (200), and (220) lattice planes. Interstitial dissolution of carbon in platinum can increase the lattice parameter of platinum and potentially produce systematic error in temperature measurements. However, the lattice parameters of the quenched platinum samples were measured to be the same as in the starting material, indicating that the diffusion of carbon didnot affect the accuracy of the temperature measurement. X-ray diffraction measurements in the temperatures range between room temperature and $1750{ }^{\circ} \mathrm{K}$ were conducted on the beam line B-2 $(\lambda=0.485946$ Á) of the Cornell High Energy Synchrotron Source (CHESS). Diffracted x-rays were collected between Bragg angles of $2 \theta=5^{\circ}$ and $2 \theta=25^{\circ}$ using a MAR3450 imaging detector. Sample to detector distance and other diffraction geometry parameters were calibrated using a $\mathrm{CeO}_{2}$ standard. 2D 
angle-dispersive diffraction images were processed using the software FIT2D to generate the intensity versus two-theta diffraction patterns. Each diffraction peak was indexed and fitted with a pseudo-Voigt function to determine its $d$-spacing.

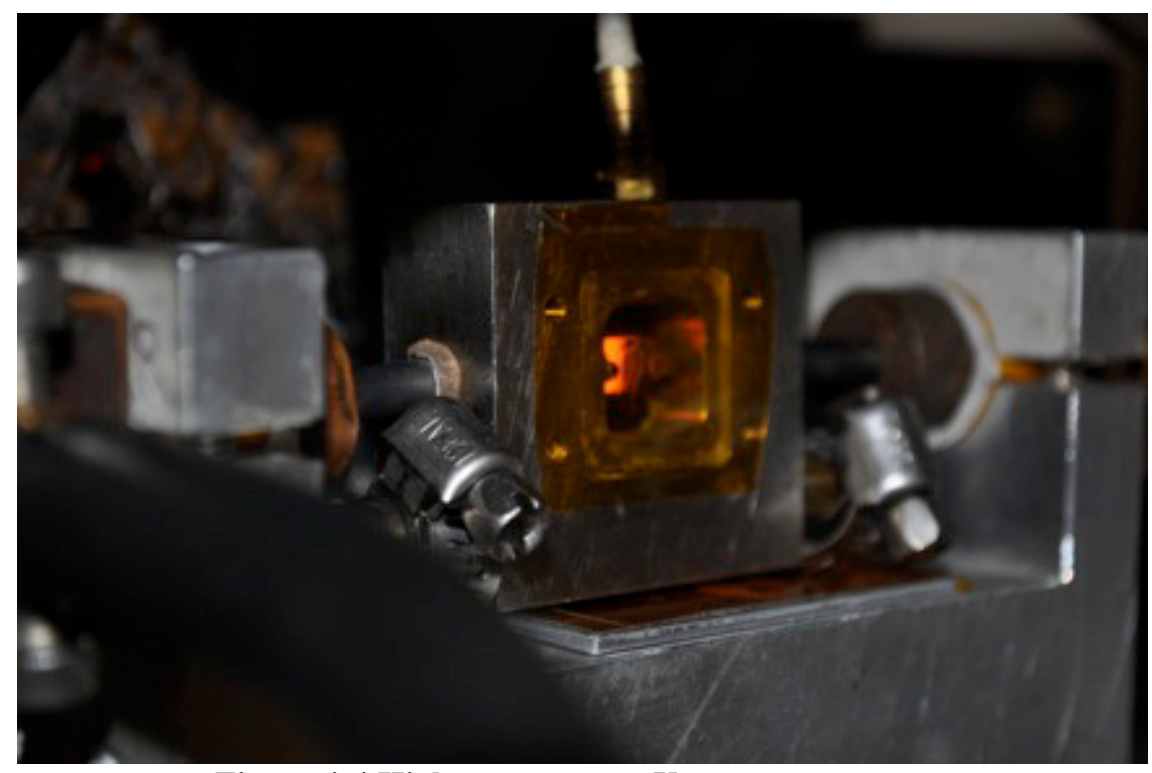

Figure 4-4 High temperature X-ray setup

X-ray beam $(100 \mu \mathrm{m}$ FWHM) was centered onto the point of interface of sample and carbon, and alternatively, onto a region of the pure sample. Diffraction patterns were collected from the platinum-carbon interface and from pure platinum at several temperatures up $1700{ }^{\circ} \mathrm{K}$ After heating, the samples were quenched by shutting off the power to the heating element and several diffraction patterns were collected from the samples at room temperature. There is no evidence of formation of any new phase or anomalous melting point in pure platinum sample

\subsubsection{Hermeticty measurement}

The present technique uses a Model 979 Series Helium Mass Spectrometer Leak Detector manufactured by Varian. A mass spectrometer tuned to helium electrical signals that are proportional to the helium concentration.. The helium system includes a vacuum pump to pull a 
vacuum on the sample and can detect helium detection to 1 X $10^{-7} \mathrm{~atm}-\mathrm{cc}-\mathrm{sec} \mathrm{He}$. A stand-alone controlled leak calibration standard, coupled with a calibrated internal standard to the machine, provided a high precision measurement of the leak rate. An O-ring leak testing fixture was manufactured with the required size of the feedthrough assembly to check hermeticity of the sample.

\subsubsection{Densification behavior}

Shrinkage of the metal powders, alumina powder and 96\% HTCC tape were measured by the horizontal optical dilatometer Misura ${ }^{\circledR}$ ODLT. Optical dilatometers use a beam of light to measure the dimensional changes and therefore the sample is not in contact with the measuring system. A laser beam is split into two beams, which are reflected by the top of the sample and focused on the top of the sample and at a high magnification image recorded on a CCD camera. Using blue light with a wavelength of $478 \mathrm{~nm}$ and magnifying up to 0.5 micron per pixel, the image will shift 1.000 pixel. A $5 \mathrm{~mm}$ packed powder sample placed vertically in the furnace can be measured the expansion or contraction with a resolution of one part over 100.000 .

\subsubsection{Thermal analyses}

TA Instruments, TA Q600 SDT instrument (simultaneous thermogravime and differential scanning calorimetry) was used to measure the calorimetric and weight lost behavior of the samples at high temperatures in air atmosphere. Alumina cups were used to heat the samples up to high temperature. Prior to the heating process in each run, the weight of two sample cups were calibrated to calculate the exact weight change of the sample. 


\section{Characterization of the powder materials}

\subsection{Platinum particles}

\subsubsection{Microstructure of platinum particles}

The high magnification picture of different platinum particle used in this study, are shown in figure 5-1.
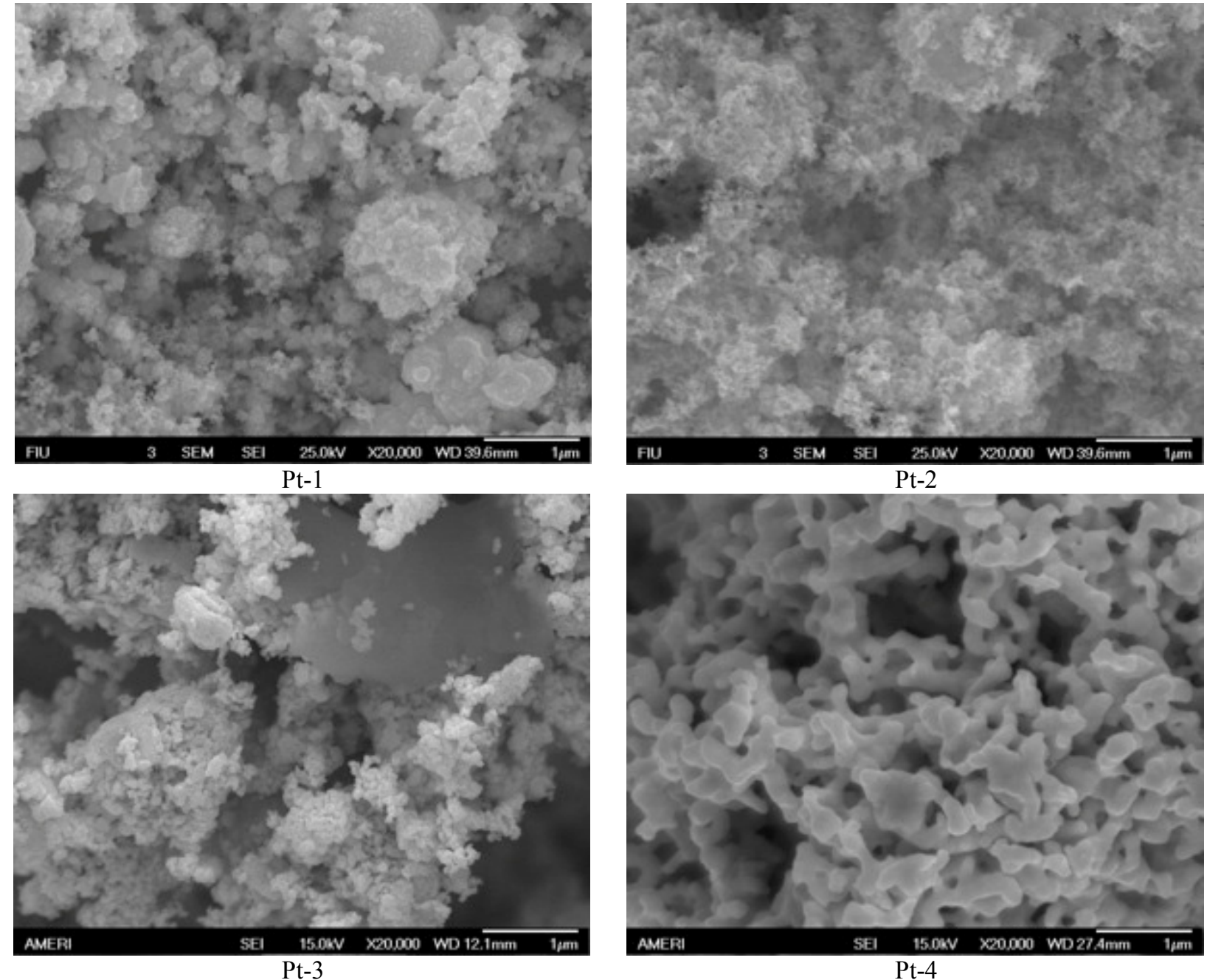

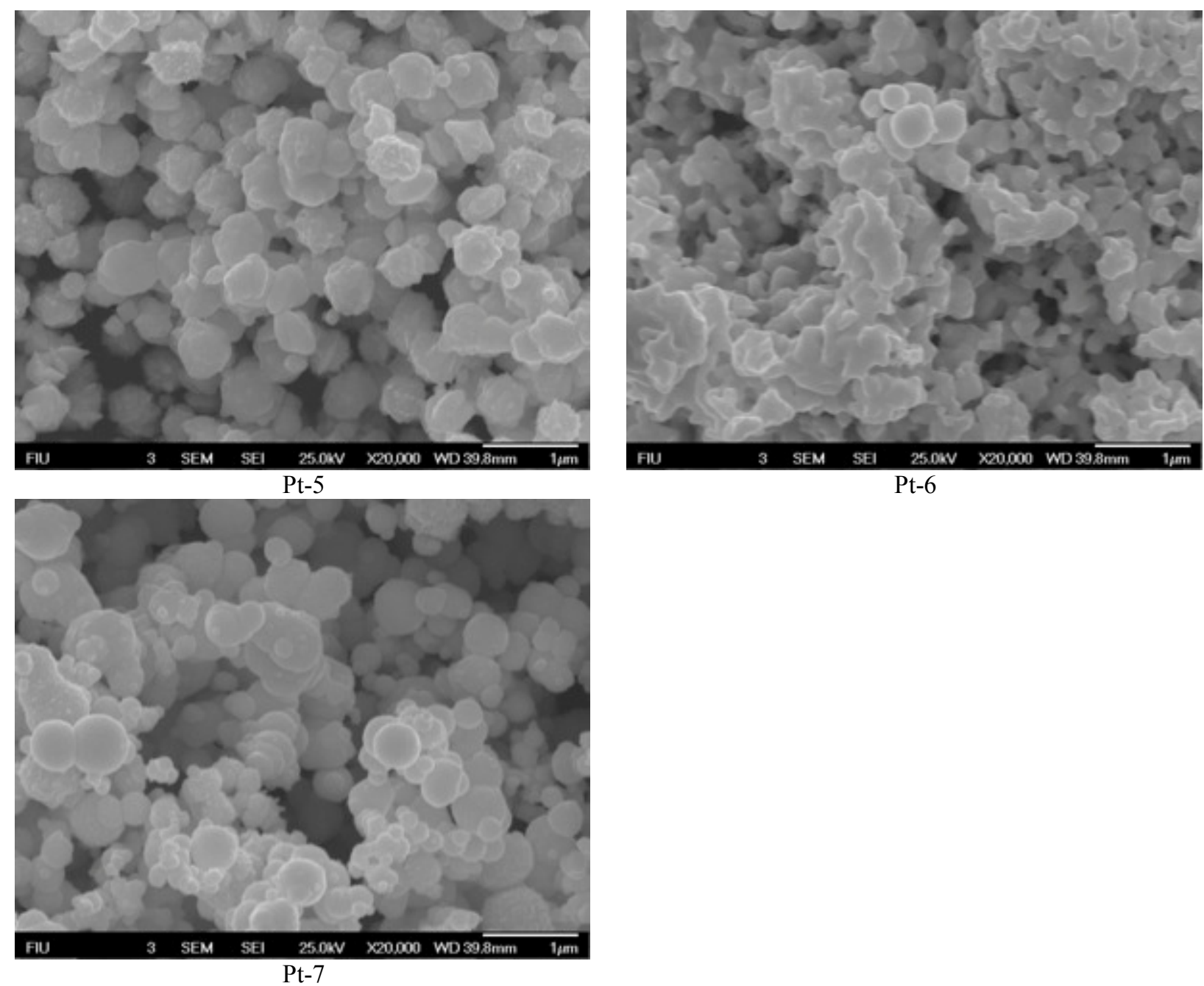

Figure 5-1 SEM image of different platinum particles used in this project

The Pt-1 and Pt-2 are both agglomerated nano particles. The Pt-1 has the lower surface area due to the formation of larger agglomerated particle, as shown in figure 5-2. Because the Pt3 particles originally came from the ready to used ink, a large glass particle shown in figure 5-1.

Even though the Pt-4 has a larger particle size and lower surface area in comparison to Pt-2, as observed from figure 5-1, it has a lot of void space, which increases the chance of shrinkage. Pt-6 and Pt-4 are originally from the same kind of platinum particle family. Their difference is in the degree of the pre-sintering process. Pt- 4 is the high surface area spray dried 
agglomerate of platinum black metal sintered into a particle. Pt-6 is pre-sintered more that Pt-4, so it has larger particle size and lower surface area, which decreases the chance of shrinkage. Pt5 and Pt-7 both looks like spherical particles. The Pt-5 demonstrates a narrower particle distribution than Pt-7.

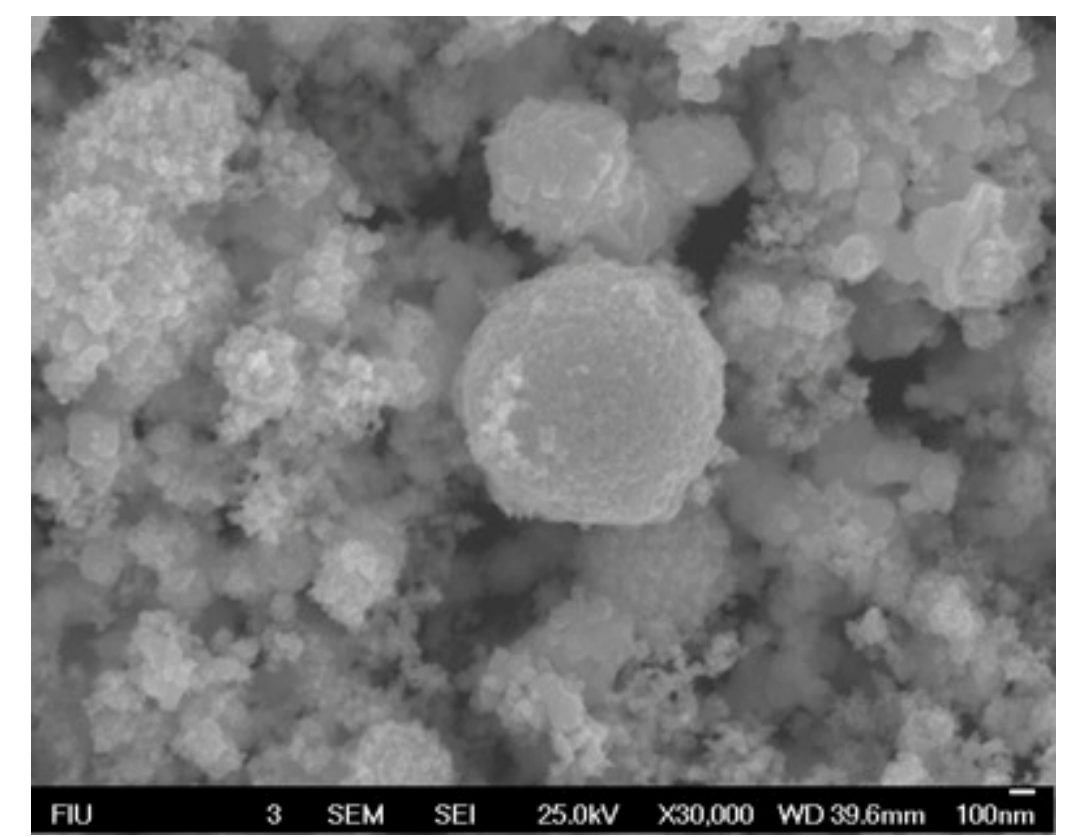

Figure 5-2 High magnification images of agglomerated Pt-1 nano particles

\subsubsection{High temperature behavior of platinum particles}

The high temperatures calorimetric and weight change behavior of different platinum particles are shown in figures 5-3 and 5-4. Powders shown in figure 5-3 are compressed up to 300 PSI and figure 5-4 demonstrates loose powders. It is clear that all powders have the initial weight lost at around $400{ }^{\circ} \mathrm{C}$, which is attributed to the decomposition of first platinum oxide and desorption of molecular oxygen. Another weight loss happens at temperature around $1150^{\circ} \mathrm{C}$. This behavior of platinum could be attributed to the decomposition of platinum dioxide and desorption of oxygen from the bulk of platinum. This oxygen layer is adsorbed on the layer of platinum below the surface and it is almost inert in temperature below $800^{\circ} \mathrm{C}[70]$. The results 
of DSC experiments are consistent with the exothermic reaction of decomposition that occurred around $1000-1200^{\circ} \mathrm{C}$. However, the compressed powders in figure 5-3 do not show much weight change. Pt-2, which has the highest surface area, shows significant sign of weight lost at high temperature. Pt-4 has the highest porosity; therefore the loose powder lost more weight at high temperature compared to the compressed powder. The calorimetric results also are consistent with these results. The heat generated by compressed particles in figure 5-3 is much less than loose powder and all of them almost show the same pattern

In general, the extent of the exothermic reaction at $1150^{\circ} \mathrm{C}$ depends on the surface area and accessibility of oxygen to the platinum particles. Pt-6 has the lowest surface area and accordingly the lowest exothermic heat generation. Pt-2 and Pt-4 have the highest heat generation. Pt-2 has the highest surface area, and Pt-4 has the most porosity that enhances the oxygen escape route.

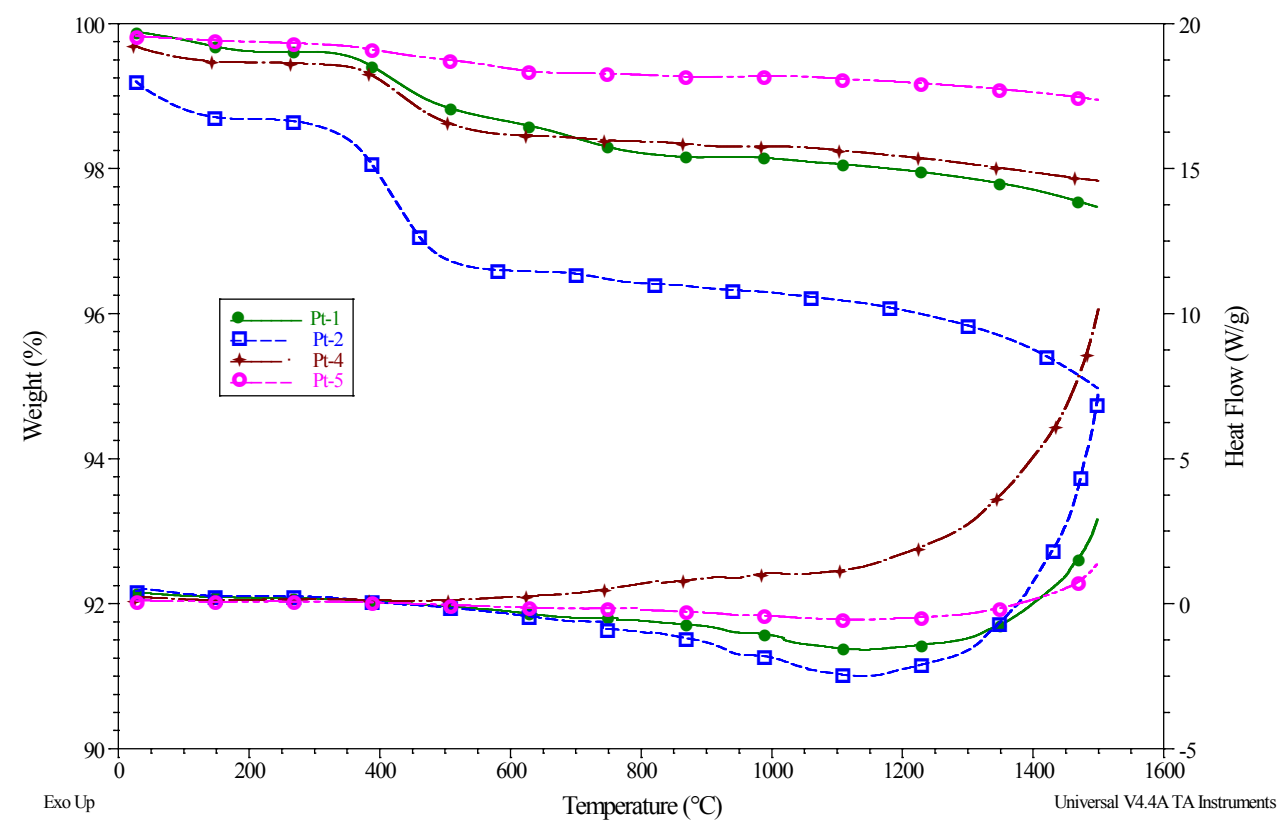

Figure 5-3 DCS and TGA results of compressed power 


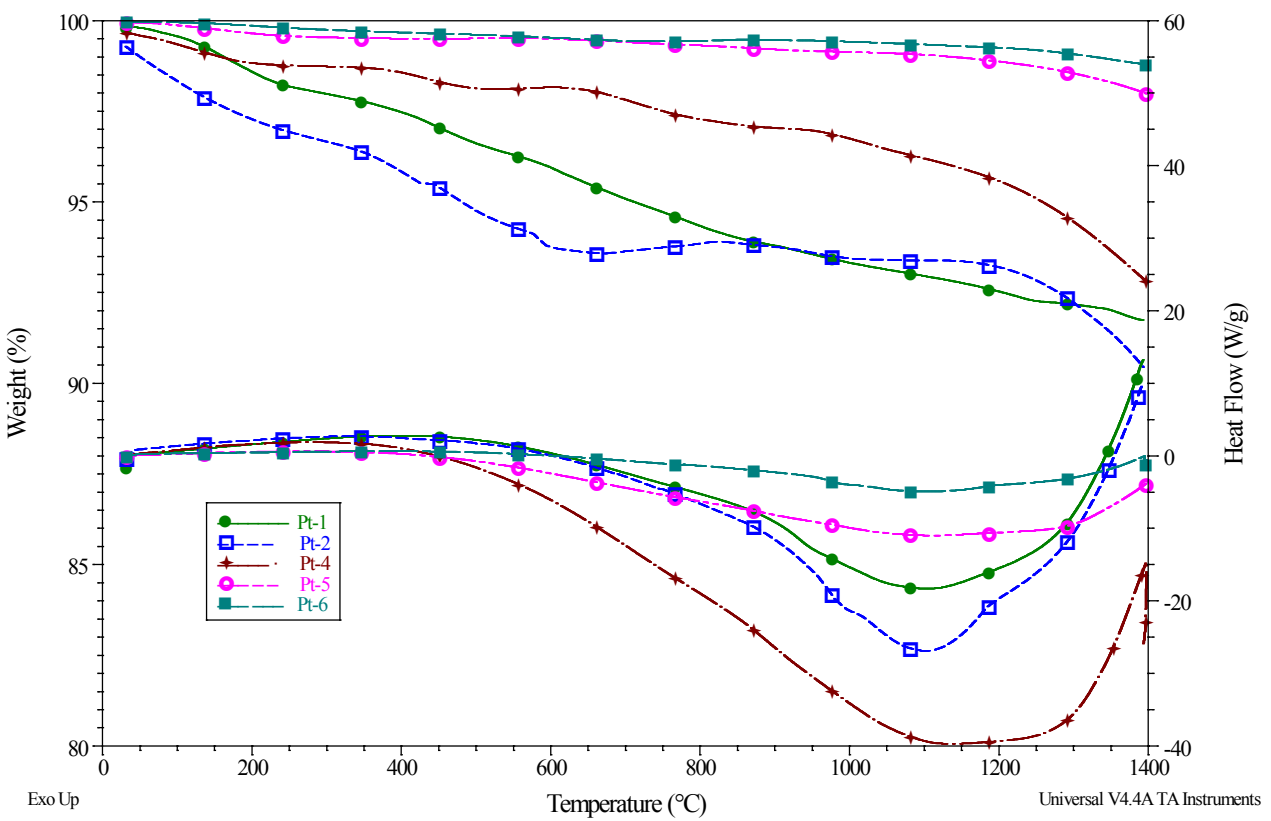

Figure 5-4 DSC and TGA results of loose powder

\subsection{Iridium microstructure and oxidation behavior}

The microstructure of the Ir powder is shown in figure 5-5. Iridium powder has a high surface area and particles' diameter are about $100 \mathrm{~nm}$.
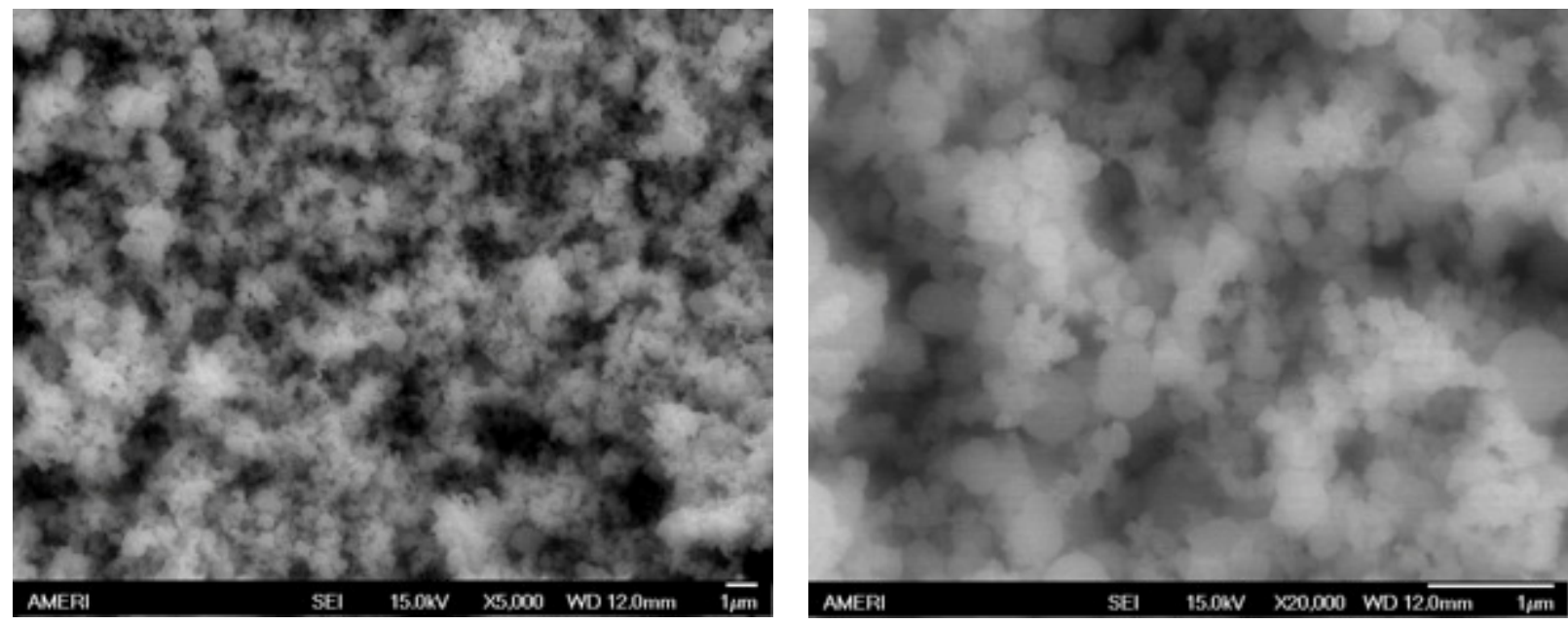

Figure 5-5 SEM image of iridium powder 
Iridium has been used as the adhesion promoter to increase the bonding strength between platinum and alumina. Iridium forms a stable oxide in temperature lower than $1000{ }^{\circ} \mathrm{C}$ and therefore the oxide bonding increases the bonding strength to alumina. Oxidation behavior of the iridium powder in high temperature has been studied by calorimetry and weight loss measurement. The iridium powder sample was heated up to $1200^{\circ} \mathrm{C}$ at the rate of $10^{\circ} \mathrm{C} / \mathrm{min}$ and was kept on that temperature for an hour. The sample then was cooled down at the same rate. To study the behavior of the sample in several heat treatments, the heating profile was repeated 3 times on the same sample. The results of the DSS and TGA experiments are shown in figure 5-6.

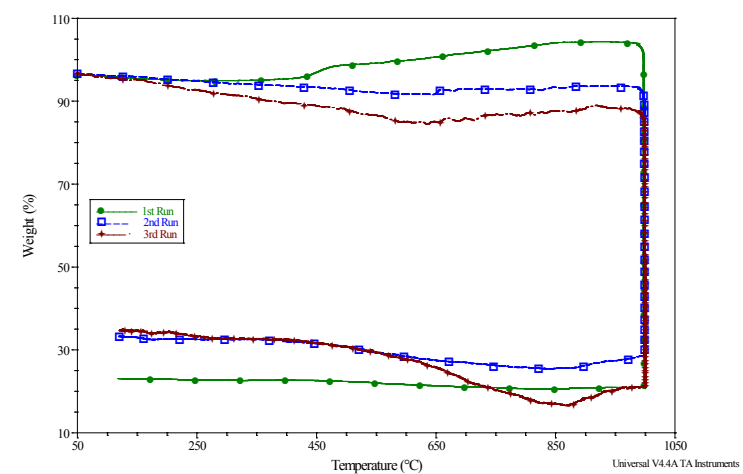

(a)

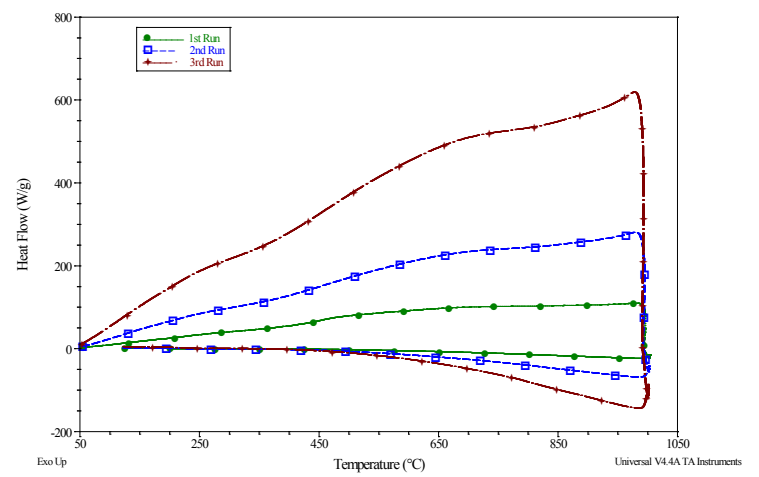

(b)

Figure 5-6 DSC and TGA results of iridium powder oxidation. (a) TGA curves, (b) DSC curves

The behavior of the iridium powder is different from the bulk metal. In every run, about $80 \%$ of the original weight of powder evaporates. Wimber et al. [143] showed that above $1100{ }^{\circ} \mathrm{C}$ iridium oxide decomposes to pure iridium and volatile iridium oxide. Keeping iridium at high temperature in oxygen produces a high amount volatile iridium oxide, which reduces the weight of the powder up to $80 \%$ in each run. In each run, iridium evaporates from the surface of the powder makes the surface more active, as indicated by the absorbed heat in the third run. Sintering and densification behavior of metal particles 
The densification behavior of different platinum particles at $1550{ }^{\circ} \mathrm{C}$ are shown in figure 57. As was expected, Pt-4 has the highest amount densification due to large porosity, and Pt-5 and Pt-7 with large spherical particles and low surface area showed the lowest amount of shrinkage and densification.

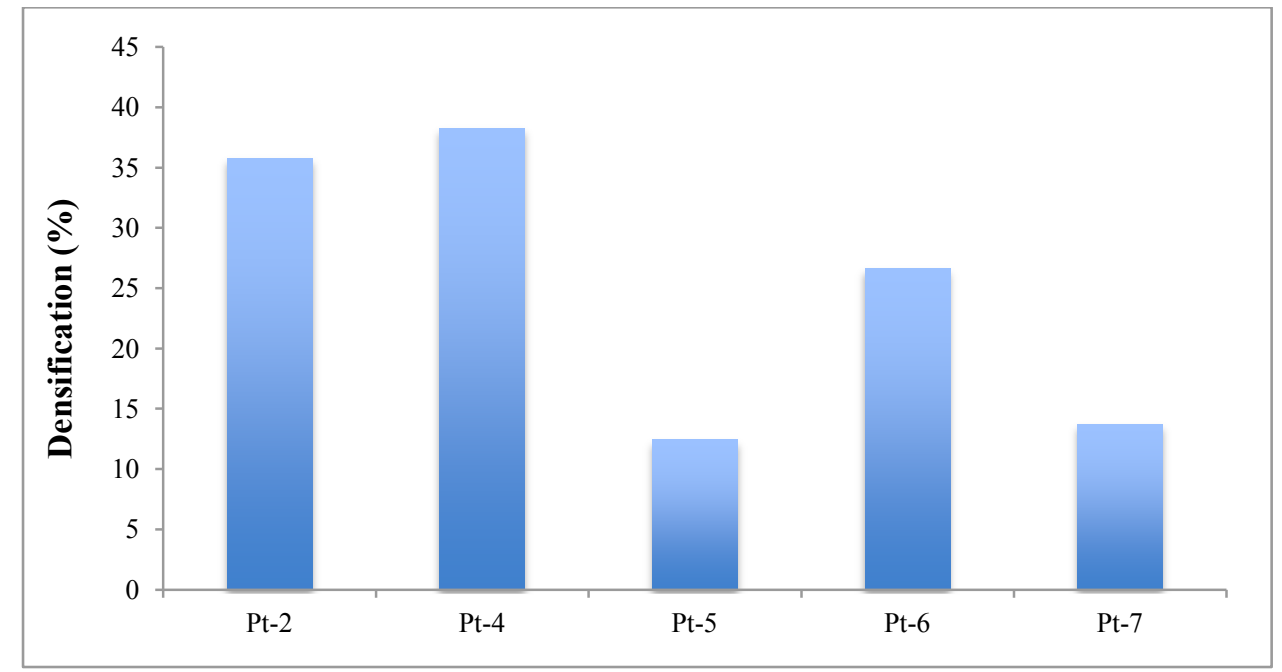

Figure 5-7 Densification behavior of different platinum particles in $1550{ }^{\circ} \mathrm{C}$

The densification rate (Shrinkage) and its derivative (der. Shrinkage) of two different platinum particles, iridium black and the combination of platinum and iridium are shown in figure 5-8. Derivative of densification rate helps to determine the critical points in the densification behavior. As depicted in figure 5-8, platinum powders densifies at lower temperature than iridium. The maximum densification rate for Pt-3 and Pt-6 happened at $625^{\circ} \mathrm{C}$ and $770{ }^{\circ} \mathrm{C}$ respectively, while iridium occurred at $1085^{\circ} \mathrm{C}$. The Pt-3 reached its maximum shrinkage around $950^{\circ} \mathrm{C}$ and after that its density increased. This increase could be due to the thermal expansion of platinum as well as the formation of platinum oxide. Platinum oxide would evaporate at temperature above $1000^{\circ} \mathrm{C}$ and could create a porous structure in platinum sample. This porous structure could expand and decrease the density as it can be seen in the figure 5-8. Iridium almost keeps it structure and shrinks once only in $1070{ }^{\circ} \mathrm{C}$. When Ir is mixed with the Pt- 
6 particle, the densification profile follows almost the same pattern as pure iridium. Even though iridium represents $20 \%$ of the composition, its solid structure inhibits the densification and the sintering of platinum. At $1500{ }^{\circ} \mathrm{C}$, Ir , Pt-Ir and Pt-6 shrinks by 78, 68 and $61 \%$ respectively.

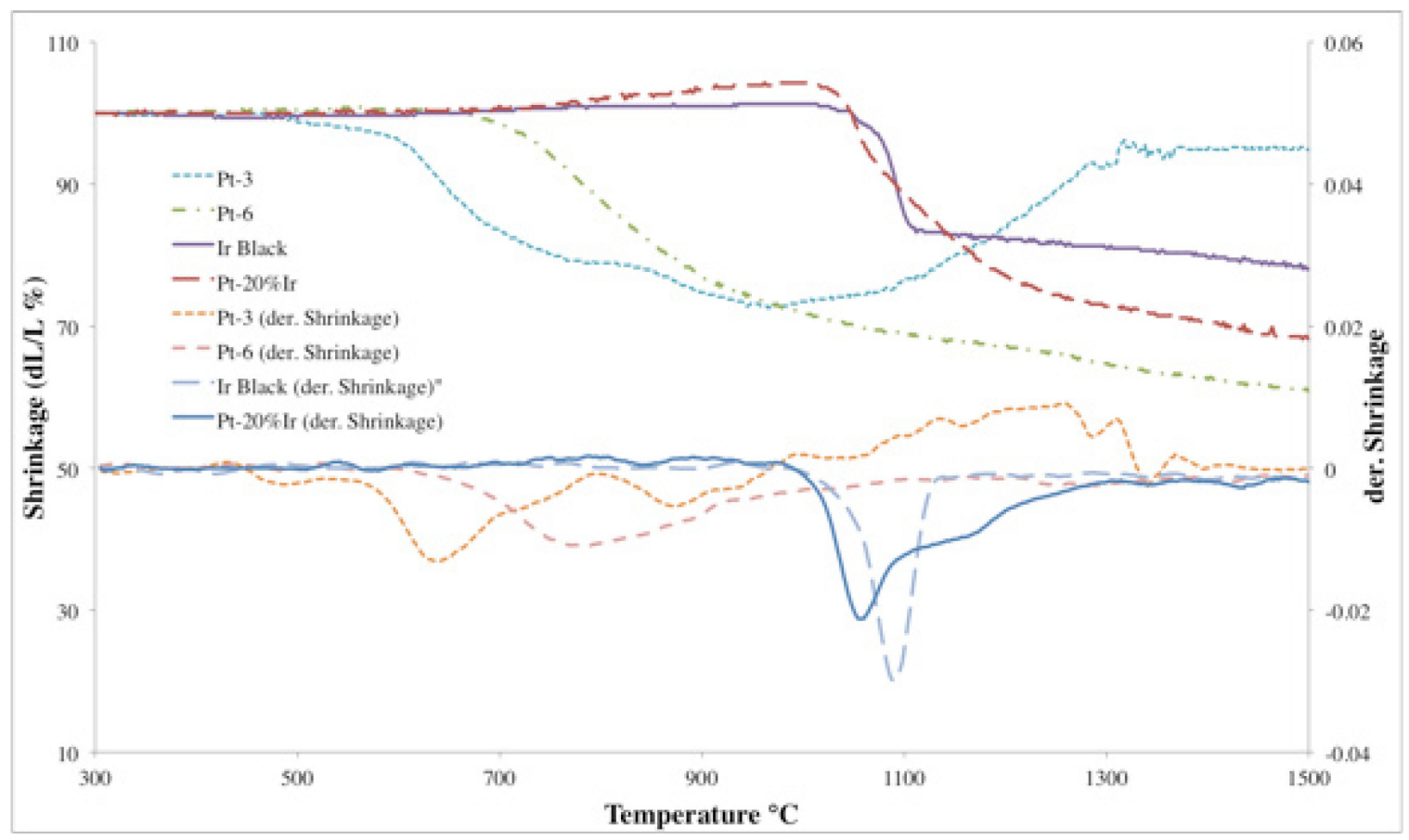

Figure 5-8 Shrinkage rate of Platinum and iridium particles

The high temperature calorimetric experiments and the weight loss measurements of Pt-6, iridium and rhodium powders are shown in figure 5-9. The platinum powder looses weight only about $5 \%$, while iridium first oxidizes and gains weight but after $1000{ }^{\circ} \mathrm{C}$ the iridium oxide starts to sublimate. The total weight loss of iridium at $1400{ }^{\circ} \mathrm{C}$ is about $40 \%$. Comparison of the densification and the weight loss behavior of iridium showed almost the same pattern. It could be concluded that the iridium powder does not densify and the change in the density could be corresponded to its weight loss. The rhodium behavior is slightly different from iridium. The rhodium powder starts to oxidize around $500{ }^{\circ} \mathrm{C}$ and it is stable up to $1100^{\circ} \mathrm{C}$. However rhodium oxide starts to evaporate after that temperature. As showed before in section 5-2, the 
iridium powder has different properties from the bulk material. The same is also true for rhodium. Higher surface area of the powder increases the oxidation and consequently the evaporation process. In this way, at the end of the process the resultant structure may have a good $\mathrm{metal} /$ ceramic adhesion as well as porous metallization structure in the via.

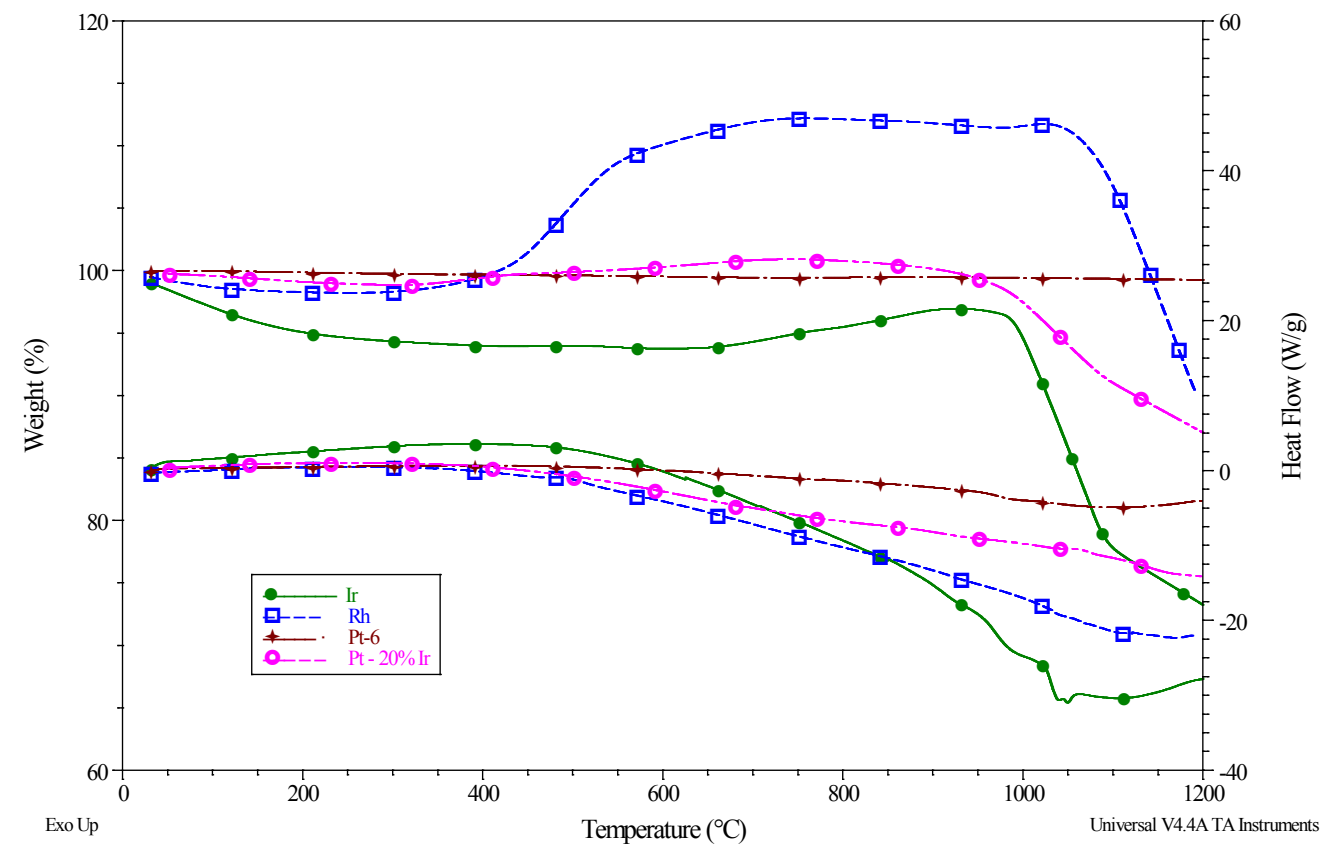

Figure 5-9 DSC and TGA results of high temperature behavior of platinum, iridium and rhodium particles

Difference in the sintering behavior of the platinum powder and ceramic materials is shown in figure 5-10. The final shrinkage of nano alumina particle is almost the same as HTCC tape, however their sintering and densification behavior are different. Nano particle densifies smoothly after the temperature reaches $1100^{\circ} \mathrm{C}$, but HTCC tape has two levels of densification. At the first step at $1100^{\circ} \mathrm{C}$ glass particles start to melt and therefore tape starts to densify. At the $1350^{\circ} \mathrm{C}$, densification increases suddenly. This behavior at $1350^{\circ} \mathrm{C}$ is supposedly due to the liquid phase sintering of glass and alumina particles. 
The shrinkage rate of metal and ceramic are significantly different. At $1500{ }^{\circ} \mathrm{C}$, the platinum powder shrinks about $35 \%$, while alumina's shrinkage was only about $17 \%$. The high difference in densification and sintering kinetics of platinum and alumina could create defects like cracks and camber in the final multilayer assembly.

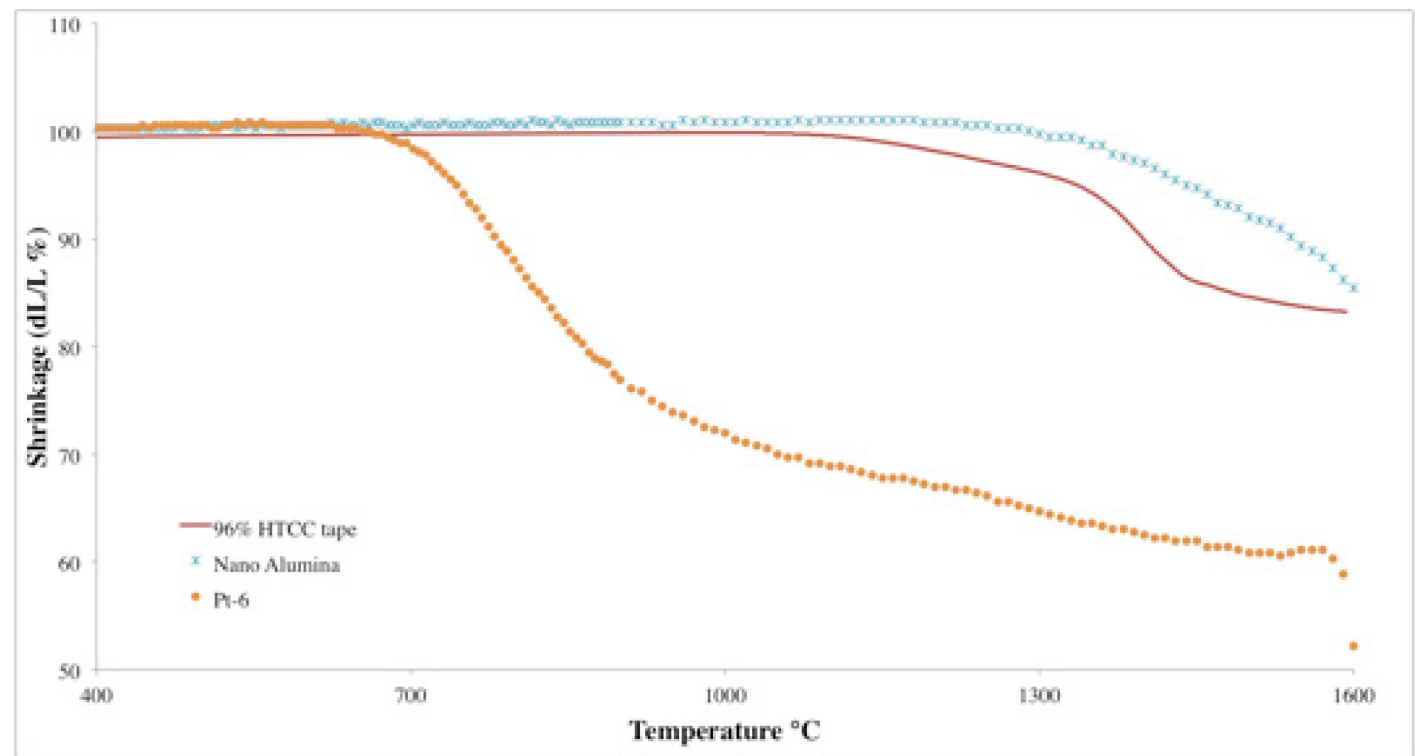

Figure 5-10 Shrinkage behavior of 96\% HTCC tape, nano alumna and Pt-6 platinum powder 


\section{Feedthrough development in natural atmosphere}

\subsection{Introduction}

As described earlier in the experimental section, punched ceramic tape was filled with platinum conductive ink, using bladder filling. The backscatter SEM image of the green ceramic feedthrough is shown in figure 6-1. It should be noted that all the samples presented in this project are prepared with Pt-4 platinum powder unless otherwise specified.
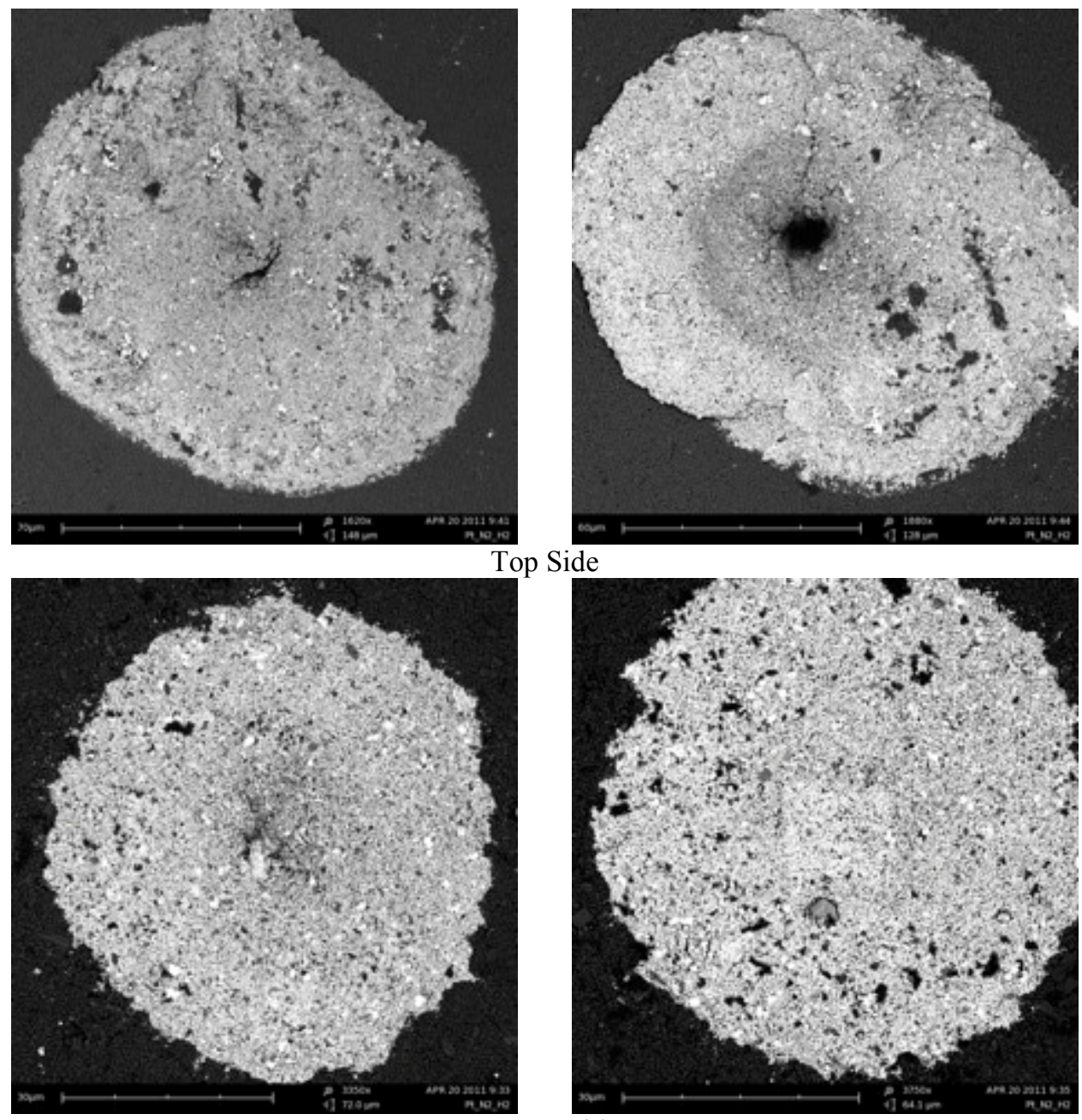

Top Side

Bottom Side

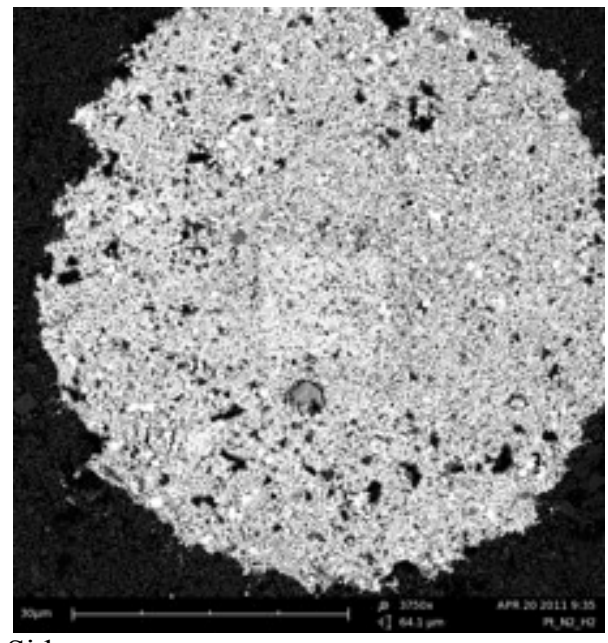

Figure 6-1 Top and bottom side backscatter SEM image of green feedthrough sample 
The bottom side of the sample looks completely dense and ink completely filled the via. However, at the topside, there is a hole or crack in the middle of the via. These kinds of defects can be due to capillary flow. Usually, because of mechanical entanglement there is a good adhesion between ink and ceramic around the wall, but capillary force can push the ink through the via and generate a hole or crack in the middle of the filled via. The size of the hole depends on the capillary force, which seems minimal in this case.

The image of the feedthrough sample after organic burn-out is shown in figure 6-2. The optical dilatometer experiment results in figure 5-8 showed that despite the size of platinum particle the densification of platinum starts after $500^{\circ} \mathrm{C}$. The dried sample shown in figure 6-2, does not have any kind of delamination or crack in the metal/ceramic interface. The comparison of the results of figures 6-1 and 6-2, indicates that the burn-out process does not have any critical effect on the sintering of platinum although carbon contamination due to incomplete or rapid burn-out processing might have some effect in higher temperatures.

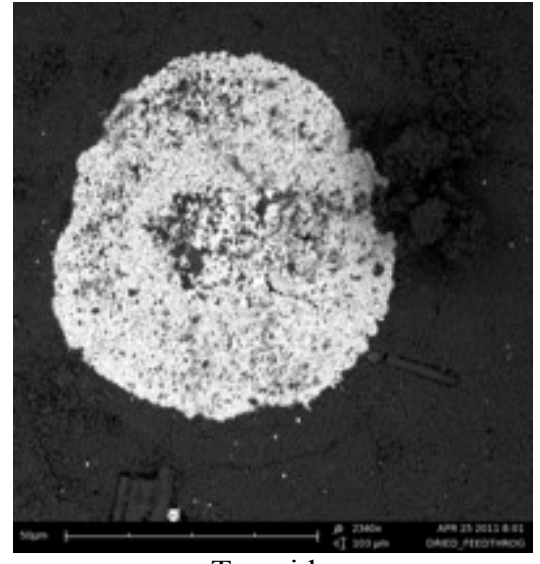

Top side

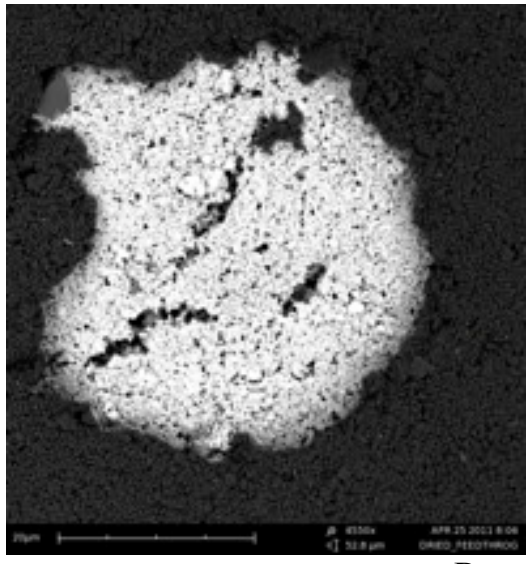

Bottom Side

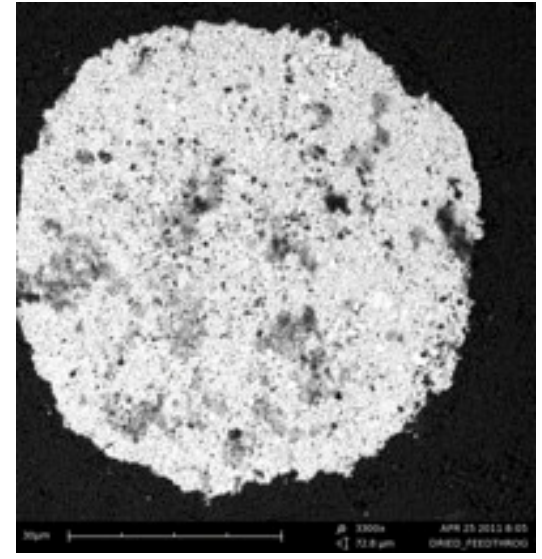

Figure 6-2 Top and bottom side backscatter SEM image of the ceramic feedthrough after organic burn-out process in $450{ }^{\circ} \mathrm{C}$

A sample via from a complete feedthrough assembly from the top and cross sectional view is shown in figures 6-3 and 6-4. As it was expected from the difference in densification 
behavior of metal and ceramic tape, a camber developed in the via and platinum delaminated from the ceramic wall. It is also clear from the figure 6-4 that platinum not only shrinks in $x-y$ direction, but also in $\mathrm{z}$ direction. This make it difficult to have a continuous electrical conductor in the via.

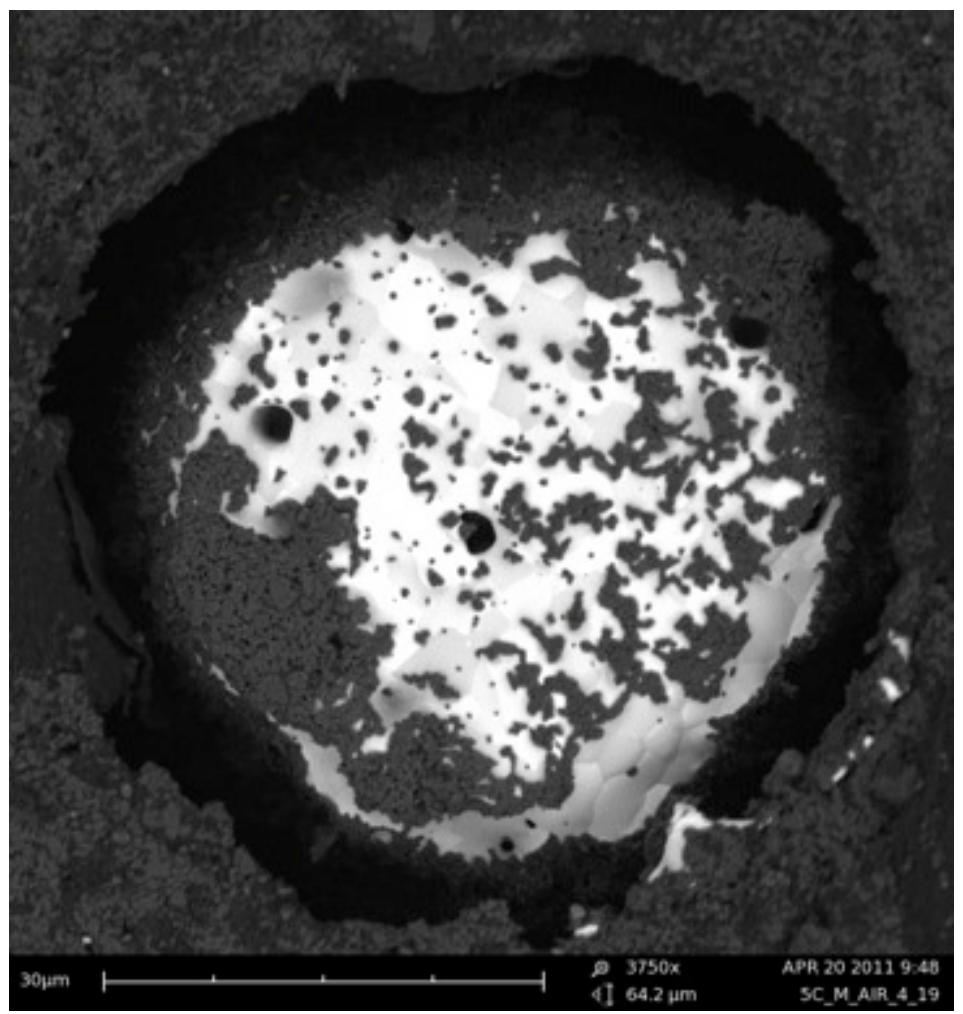

Figure 6-3 Backscatter SEM image of fired a via in ceramic feedthrough

One of the most interesting aspects of the resultant via structure is the presence of ceramic materials around the platinum. A layer of ceramic also covered the platinum via through the whole length of the via as can be seen in figure 6-3 (c). As discussed in section 2.2.1.1, the metal/ceramic bonding can be physical or chemical in nature. Glass migration from the ceramic to the metal could create a strong physical bond while formation of a platinum-alumina complex could also create a metal/ceramic bond. Platinum aluminate (i.e. $\mathrm{Pt}_{\mathrm{x}} \mathrm{Al}_{\mathrm{y}} \mathrm{O}_{\mathrm{z}}$ ) structure, as reported by Hwang et. al. [12] and Luo et. al. [16], could be formed. In addition, the remaining carbon in 
the system could also act as a reducing agent in the system. This carbon reduces alumina to form a platinum-aluminum compound. In either the case of physical or chemical interaction, platinum tries to dissolve alumina. However, the interaction force is not enough, which makes the ceramic to tear apart in the interface area as it shown figure 6-4.

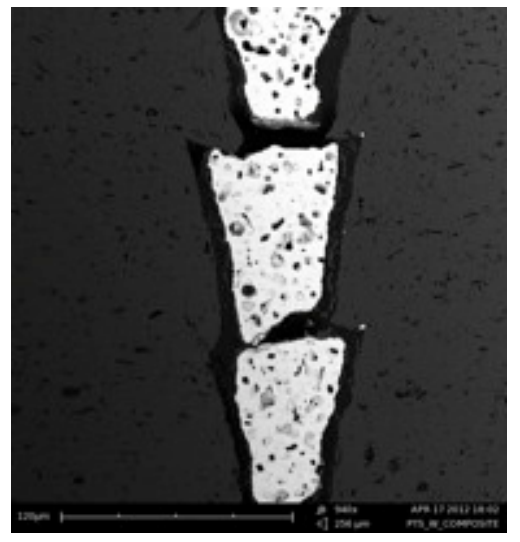

(a)

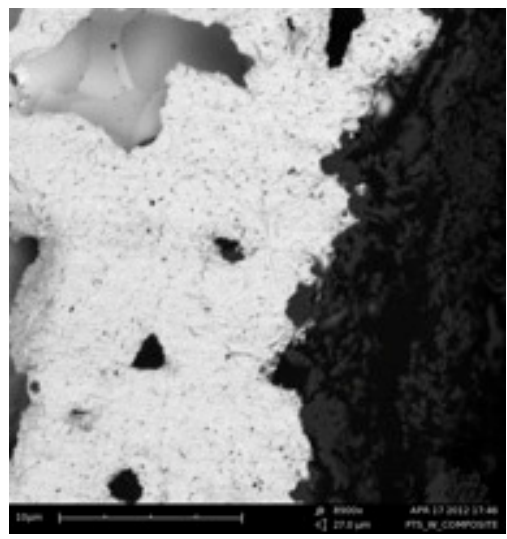

(b)

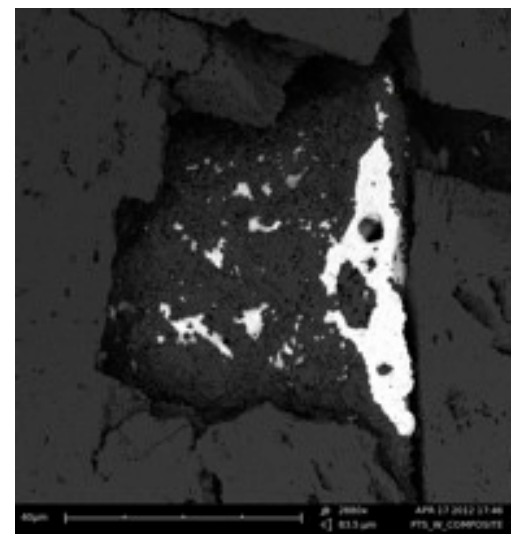

(c)

Figure 6-4 Backscatter SEM cross section image of platinum via

As shown in figure 6-3, the platinum powder densifies completely. The grain size of the platinum after firing is about $10 \mu \mathrm{m}$. This structure of platinum is similar to the molten structure, which could be attributed to the small platinum particle size. It should be noted that the hole, which can be seen in the samples, attributed to the formation of volatile platinum oxide which is usually formed in temperatures above $650^{\circ} \mathrm{C}$ [133] or the formation of the volatile carbon structure around $1000^{\circ} \mathrm{C}$.

\subsection{Platinum particle size}

As indicated in figure 5-7, different platinum powders have different densification behavior at high temperature. Because of that, conductive ink with different platinum powders were prepared and used to develop the feedthrough. The microstructures of the resultant feedthroughs are shown in figure 6-5. 

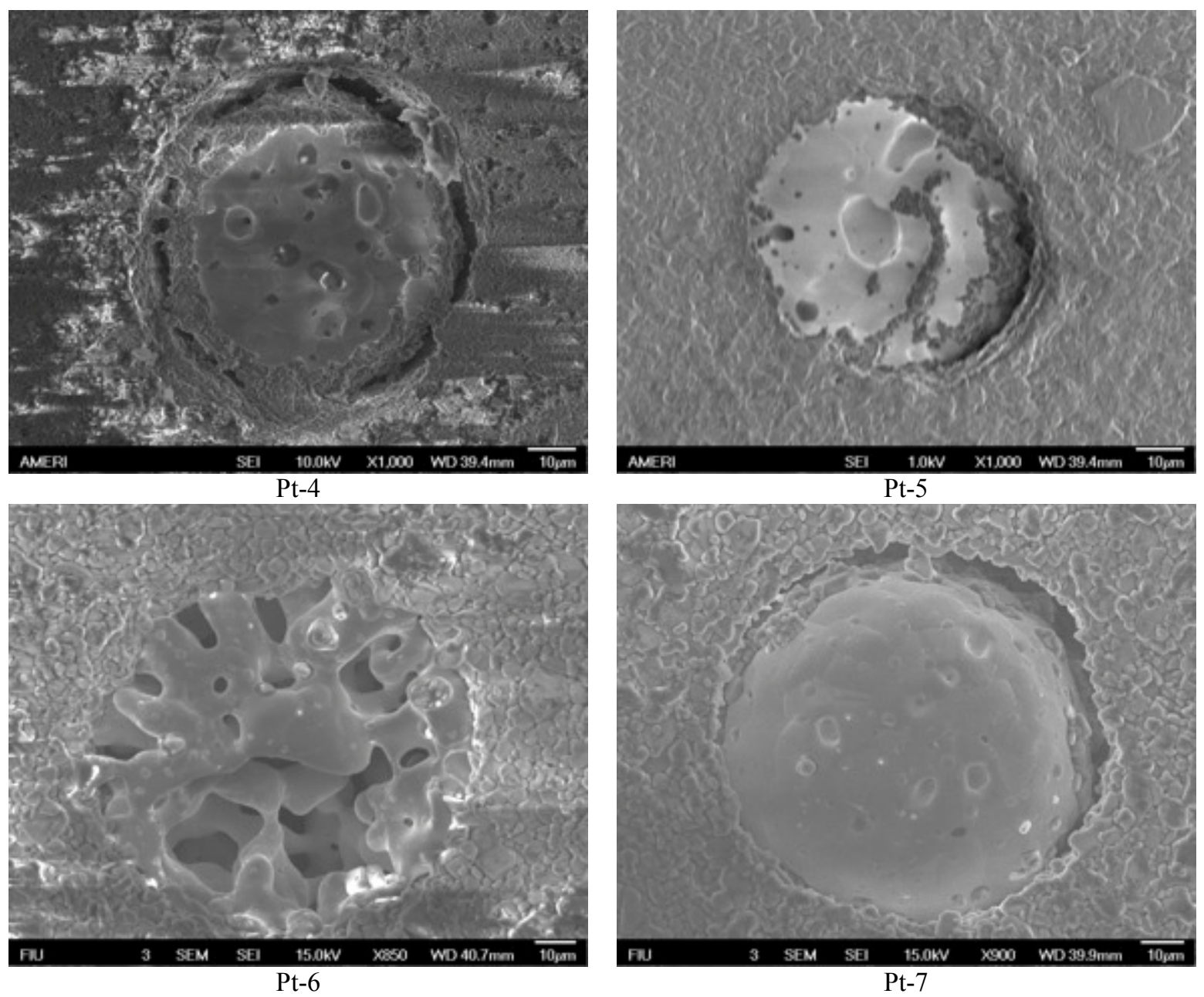

Figure 6-5 SEM image of platinum via with different platinum partcile size. Feedthroughs are fired in air at $1550{ }^{\circ} \mathrm{C}$

Despite the difference in particle size and surface area of the different powders, platinum delaminated from the ceramic and all the samples showed camber development. Pt-5 and Pt-7 have the least amount of shrinkage, which is in agreement with the results showed in densification behavior. Both Pt-5 and Pt-7 samples shrank and pulled back from the walls, yet the shrinkage is much less than the Pt-4 sample. The microstructure of the Pt-6 sample is completely different from the others. Platinum attached to the sidewalls completely, yet it has not densified. It seems that the adhesion energy between platinum and alumina is greater than the 
platinum grains cohesion energy, since Pt-6 did not made a dense metal via structure. The surrounding area of the Pt-4 powder is covered with the ceramic material, which indicates that among all particles, Pt-4 has the most amount of reaction with ceramic.

\subsection{Firing atmosphere}

\subsubsection{Air and argon atmosphere}

As discussed in section 3.11.3, sintering atmosphere has an important effect on the densification and sintering of both metal and ceramic, even though it is more critical for metals. In addition to the densification behavior, replacing air with a neutral atmosphere, such as argon, could eliminate evaporation of platinum. The atmosphere condition is the most important factor to control the interface of platinum and alumina. Electron microscope images of the samples fired in the air and argon atmospheres, at heating rate of $5^{\circ} \mathrm{C} / \mathrm{min}$, are showed in figure 6-6.

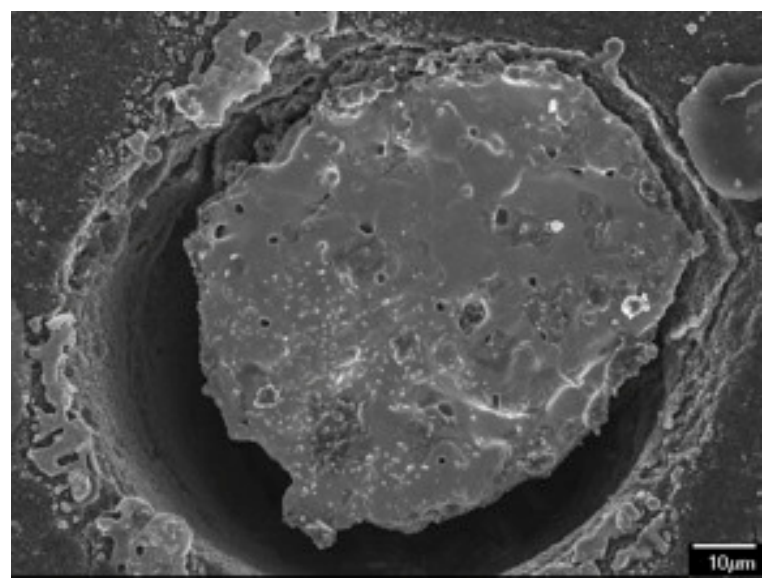

Ar

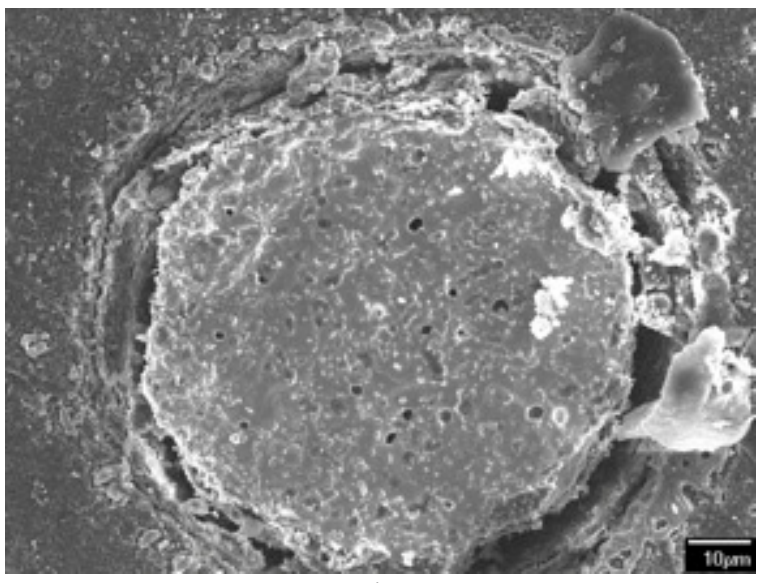

Air

Figure 6-6 SEM image of feedthrough fired in air and argon atmosphere at $1550 \mathrm{C}$.

Platinum densified in argon atmosphere more than air, as demonstrated in platinum/alumina supported catalyst system[130]. This behavior of platinum is attributed to the decomposition of platinum dioxide and to the desorption of oxygen from the bulk platinum. This 
oxygen layer is adsorbed on the layer of platinum below the surface and it is almost inert in the temperature below $800^{\circ} \mathrm{C}$ [70]. The results of DSC and TGA experiments of the feedthrough assembly in air and in argon are shown in figure 6-8. The calorimetric experiment followed the same firing profile as was used in the sintering of feedthroughs. There are two important points distinguished in figure 6-8. Even though the organic binder in the argon does not have the possibility to burn-out, it will evaporate from the sample in higher temperature so the final weight loss of the sample in both atmospheres is the same.

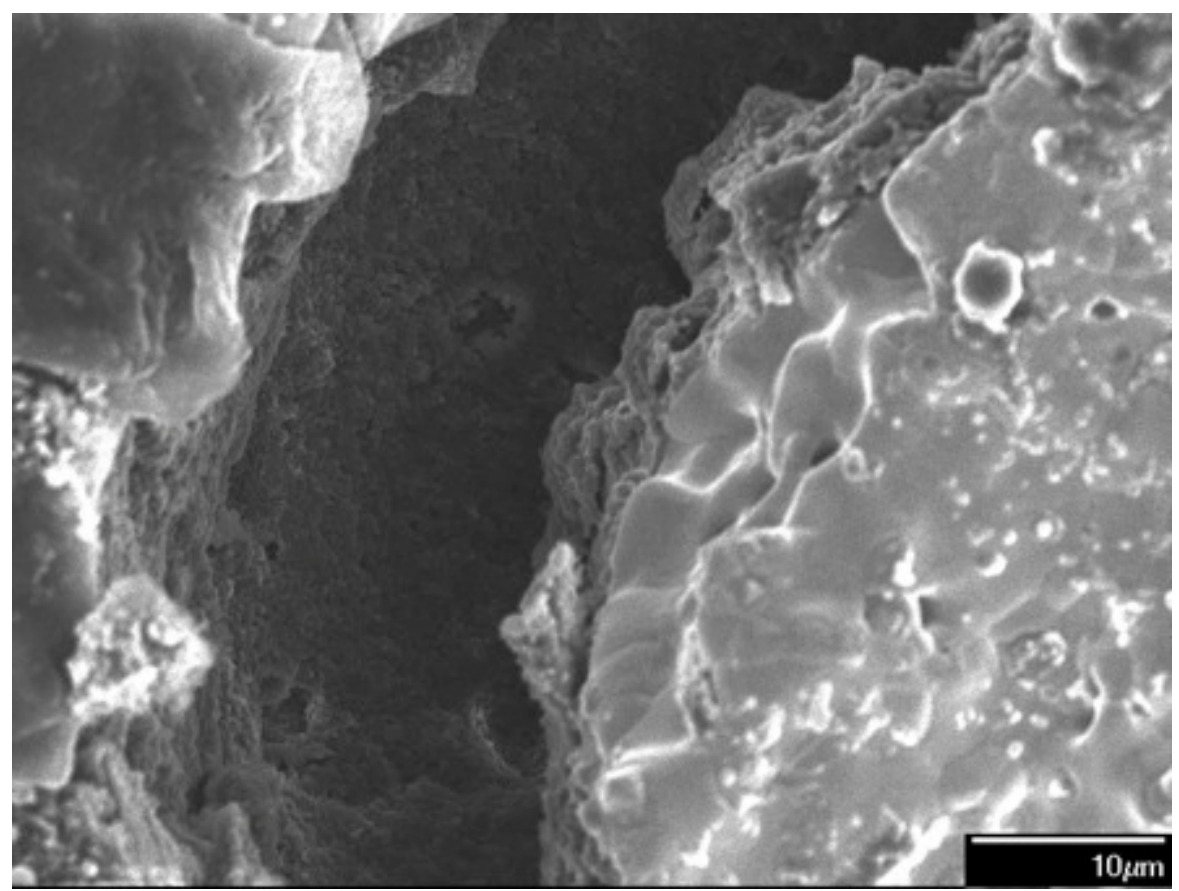

Figure 6-7 High magnification microscopic image of feedthrough fired in the argon atmosphere

The low oxygen partial pressure in argon increases the possibility of exothermic reaction of platinum oxide decomposition in argon atmosphere. The results in figure 6-8 showed that this reaction starts at $600^{\circ} \mathrm{C}$ and reaches its maximum at $1074{ }^{\circ} \mathrm{C}$ in argon, almost $60^{\circ} \mathrm{C}$ less than the temperature reaction in air, which is $1130^{\circ} \mathrm{C}$. The generated heat in argon and air atmospheres are $28.71 \mathrm{~kJ} / \mathrm{g}$ and $13.28 \mathrm{~kJ} / \mathrm{g}$ respectively. This difference in generated heat 
possibly results in local super heating in the sample and an increase in the shrinkage of the platinum. The lower temperature reaction in argon than air increases the speed of contraction and densification of platinum, so the metal has insufficient time to interaction with the ceramic. The sidewalls of the metal are very clean and exhibit no sign of reaction between the metal and ceramic in the argon atmosphere, as shown in figure 6-7.

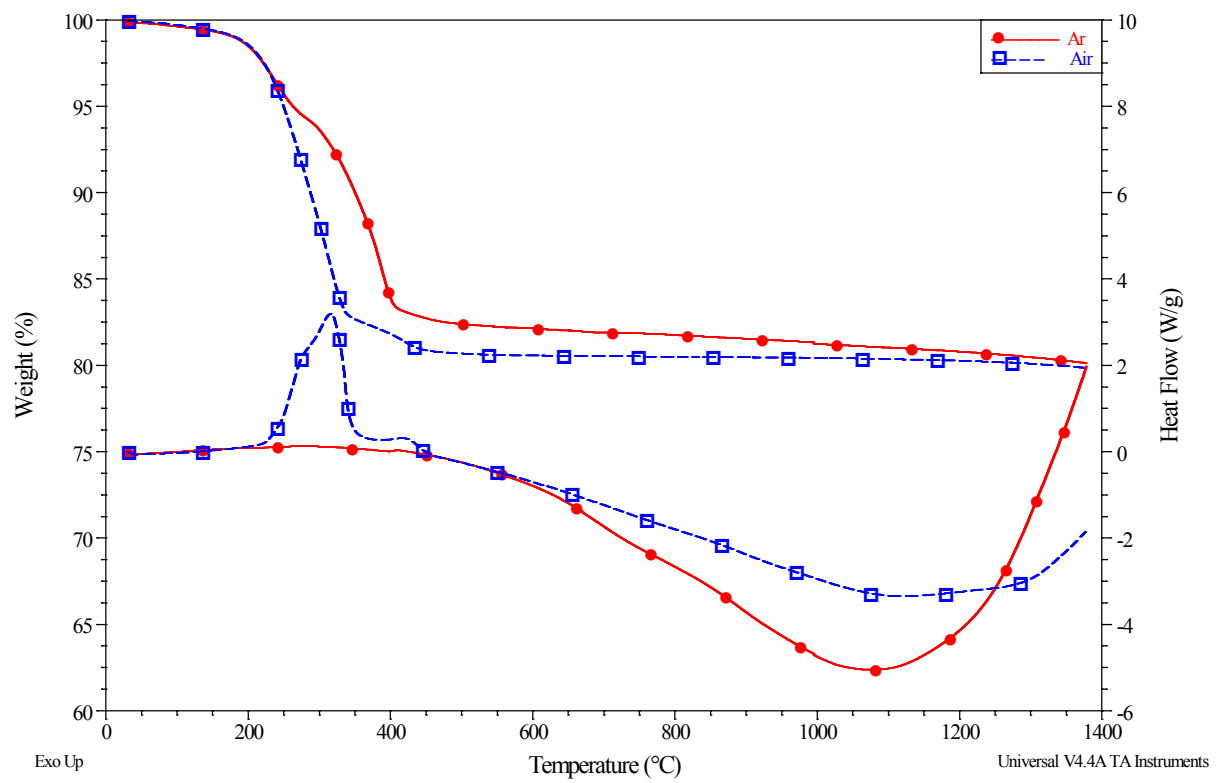

Figure 6-8 DSC and TGA results of firing feedthrough in air and argon atmosphere

\subsubsection{Vacuum atmosphere}

A feedthrough sample was fired in a vacuum atmosphere with the same firing profile used to fire the sample in air and argon. The results of the sample fired in the vacuum are shown in figure 6-9. 

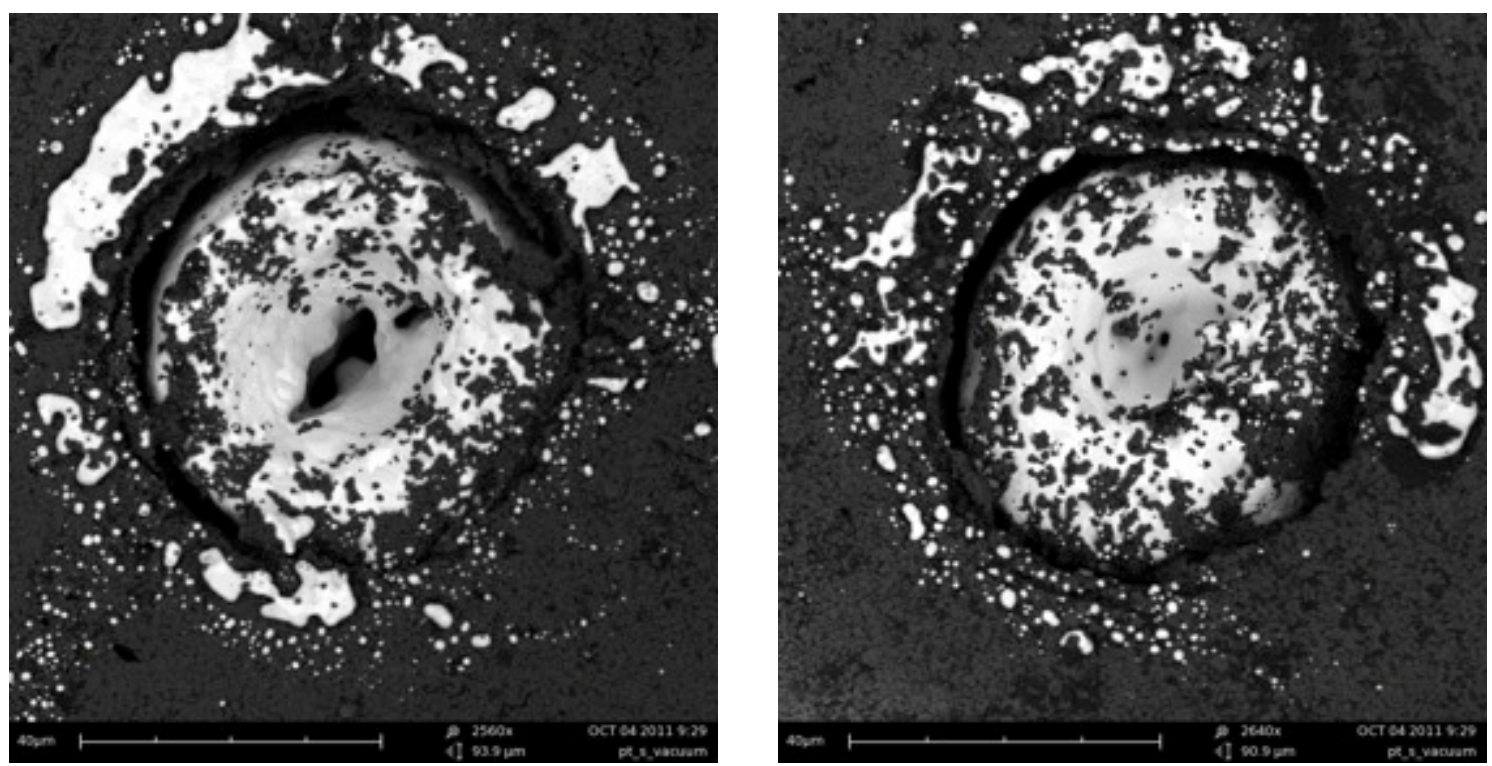

Figure 6-9 Backscatter SEM image of feedthrough fired in vacuum

The densification behavior and metal/ceramic reaction in vacuum is not that much different than that in air and argon. The densification of the fired sample is very similar to the sample fired in air. However, the sidewalls of the platinum does not exhibit physical or chemical interaction between metal and ceramic, which is very similar to the sample fired in the argon atmosphere. This result is expected due to the low oxygen partial pressure of the vacuum.

\subsection{Heating rate}

As mentioned in section 3.11.1, the heating rate is important in the densification behavior and the final density. Because of the natural shrinkage difference between platinum and alumina, controlling the heating rate may decrease the chance of delamination and camber development. Figure 6-10 indicates the DSC and TGA results of a final feedthrough assembly heated up to $1500{ }^{\circ} \mathrm{C}$ at two different heating rates.

The final weight of the samples for 5 and $2{ }^{\circ} \mathrm{C} / \mathrm{min}$ heating rate are $79.71 \%$ and $78.93 \%$ of the original weight respectively. This indicates that the weight loss of the sample is independent 
of the heating rate, yet the heat generation is heat-rate dependent. The first burn-out process happens around $150^{\circ} \mathrm{C}$, which is attributed to the organic solvent component of the organic vehicle of the conductive ink. Carbon produced by organic burn-out process could react with platinum due to the catalytic nature of platinum particle $[144,145]$. Increasing the heating rate from $2{ }^{\circ} \mathrm{C} / \mathrm{min}$ to $5^{\circ} \mathrm{C} / \mathrm{min}$, increase the heat of reaction from $1.05 \mathrm{~kJ} / \mathrm{g}$ to $2.23 \mathrm{~kJ} / \mathrm{g}$, which could increase the chance of carbon/platinum interaction. Carbon can dissolve in platinum and create the solid solution, which reduces the melting point of platinum [146]. Additionally, this interaction could enhance the sintering of platinum particles and increase the shrinkage of platinum. As mentioned before, platinum oxide decomposes around $1000^{\circ} \mathrm{C}$. Increasing the heating rate increases the amount of required heat for the exothermic reaction of platinum oxide decomposition, possibly due to the introduction of carbon into platinum. Carbon-platinum mixture creates a solid solution system, which helps carbon stability up to $1000^{\circ} \mathrm{C}$. Above that temperature, carbon could evaporate in either forms of pure carbon or carbon dioxide.

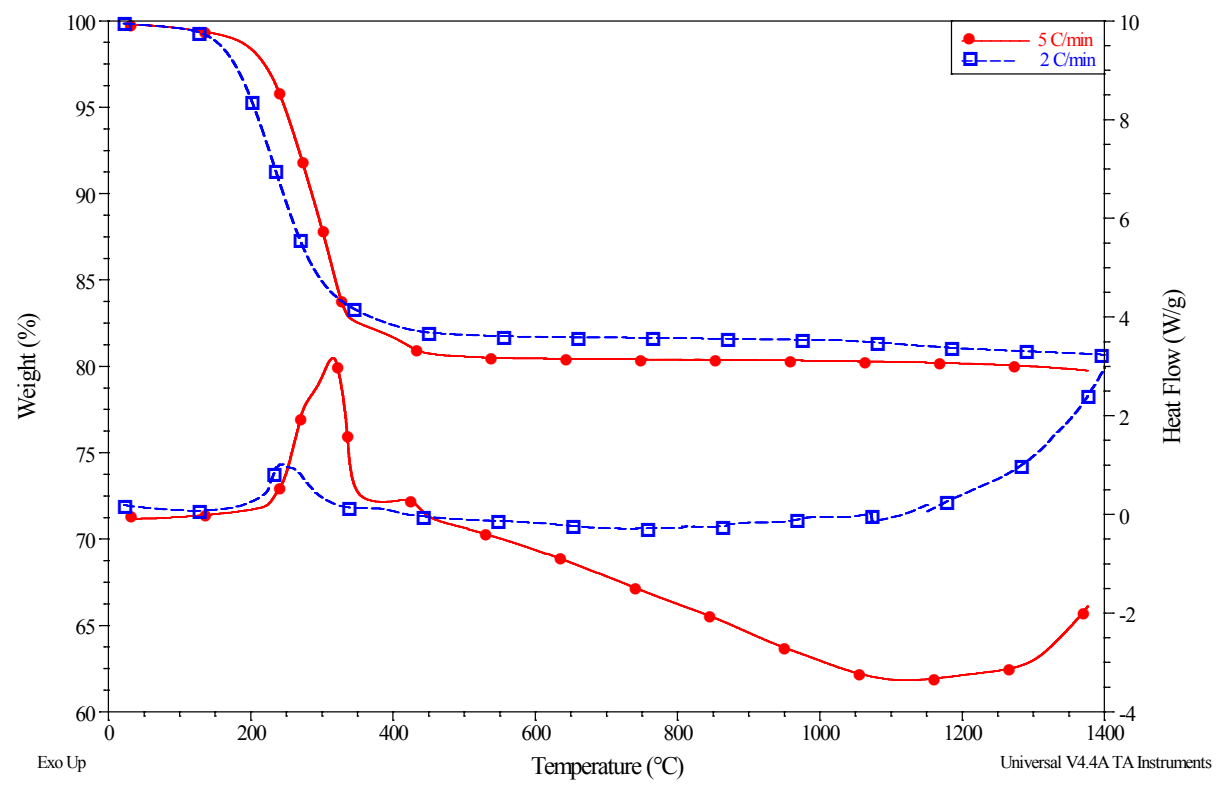

Figure 6-10 DSC and TGA results of firing feedthrough with different heating rate in air 
One of the interesting points about the DSC curve is the second burn-out point at $400{ }^{\circ} \mathrm{C}$, which is attributed to the binder phase of the organic vehicle in the ceramic tape. The heat generation of both heating rates is almost the same, which suggests the independency of ceramic tape from the heating rate. This result does not rule out any probability of the independency of ceramic burn-out cycle from heating rate, it simply suggests that at the heating rates of less than $5{ }^{\circ} \mathrm{C} / \mathrm{min}$, the burn-out cycle for HTCC tape is independent of heating rate.

Densification of the conductive ink in the via is a fairly straightforward process and is basically dependent on the sintering behavior of the metal powder. However, determination of the densification of the ceramic tape in the feedthrough assembly is complicated. Because of the presence of the vias (holes) in the ceramic tape, when the ceramic starts to densify, it contracts unto itself, resulting in an increase in the hole diameter. On the other hand, decreasing the densification of the HTCC tape decreases the shrinkage of the via and leads to an increase in the final via diameter. So the via diameter is basically dependent on the contraction force of the ceramic which depends on densification behavior. In this way the heating rate could change the densification of the tape and ink, and at the end of the process decreases the chance of failure. SEM images of feedthrough assembly fired in four different heating rates are shown in figure 611 and a summary of their densification results is also showed in table 6-1. Each measurement in the table $6-1$ is the average of the $5-10$ vias in one sample.

All of the four samples showed delamination of the metal from the ceramic and camber generation. Still, the heating rate around $5^{\circ} \mathrm{C} / \mathrm{min}$ showed the minimum difference between platinum and alumina densification. In contrast to the HTCC tape, decreasing the heating decreases diameter of platinum via. Decreasing the heating rate increases the shrinkage of the alumina. The interaction of platinum and alumina can increase the adhesion force. Low heating 
rate can create the sufficient time for diffusion of platinum to alumina and alumina to platinum and increases their physical adhesion.
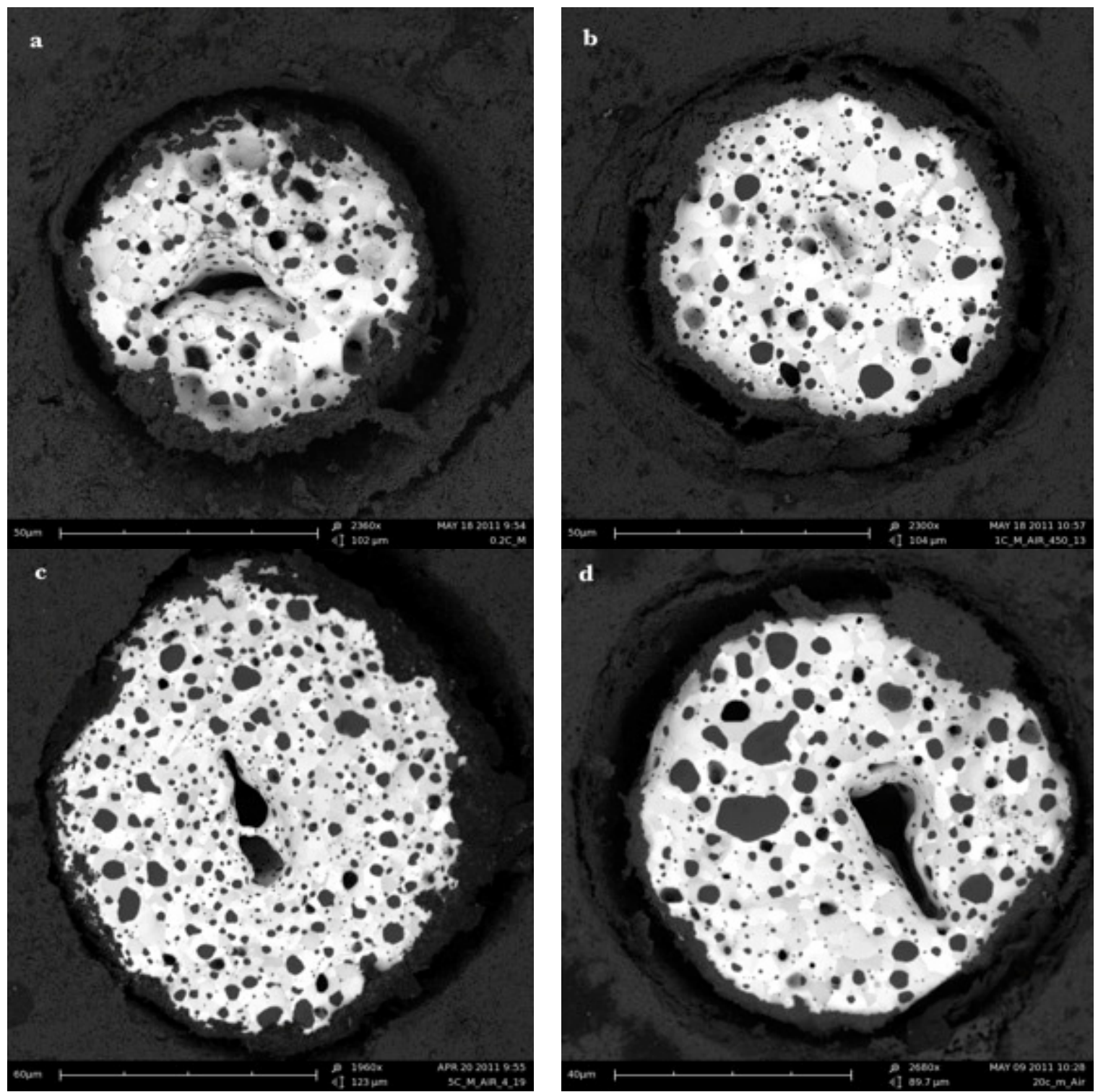

Figure 6-11 Backscatter SEM image of the platinum via fired in different heating rate. a) $0.2^{\circ} \mathrm{C} / \mathrm{min}$, b) $1^{\circ} \mathrm{C} / \mathrm{min}$, c) $5^{\circ} \mathrm{C} / \mathrm{min}$ and d) $20^{\circ} \mathrm{C} / \mathrm{min}$.

It could be concluded that a slow heating rate is necessary for the binder burn-out process.

This slow heating rate allows organic binder to evaporate before any interaction with platinum.

However, very low heating rate at high temperatures increases the densification of platinum and 
increases the chance of camber and other failures development. The optimum heating firing profile consists of $1-2^{\circ} \mathrm{C} / \mathrm{min}$ up to $450{ }^{\circ} \mathrm{C}$ for binder burn-out and $5-10^{\circ} \mathrm{C} / \mathrm{min}$ up to $1550{ }^{\circ} \mathrm{C}$ for sintering of the materials.

Table 6-1 Densification behavior of platinum and alumina in different heating rates.

\begin{tabular}{cccc}
\hline Heating rate $\left({ }^{\circ} \mathbf{C} / \mathbf{m i n}\right)$ & Platinum $(\boldsymbol{\mu m})$ & Alumina $(\boldsymbol{\mu m})$ & Difference $(\boldsymbol{\mu m})$ \\
\hline $\mathbf{0 . 2}$ & 64.068 & 86.891 & 22.822 \\
$\mathbf{1}$ & 66.287 & 87.707 & 21.42 \\
$\mathbf{5}$ & 68.36 & 82.37 & 14.01 \\
$\mathbf{2 0}$ & 69.775 & 88.043 & 18.268 \\
\hline
\end{tabular}

\subsection{Glass migration}

The physical bonding in the metal/ceramic interface is a very important mechanism to ensure the sealing and hermeticity of the final product. The migration of the glass from the ceramic to the metal by a liquid diffusion mechanism is the critical parameter to improve the metal/ceramic bonding. As mentioned in section 2.2.2, the migration of the glass is due to capillary force from the ceramic to the metal. This capillary force primarily depends on the difference in metal and ceramic surface tension and particle size. At high temperatures, capillary force increases by decreasing surface tension of metal/glass [69]. However, in the case of platinum, the metal/glass surface tension increases due to the decomposition of platinum oxide. This behavior of platinum could make some additional pressure to move the glass out of the metal and float it on top of the metal. To study this behavior, backscatter and secondary electron images of one sample via was taken and EDS analyses of different points was done to study the elemental composition distribution on the surface of the via. The SEM image of a sample via and its EDS elemental analyses of four different surface points are shown in figure 6-12 and 6-13. 


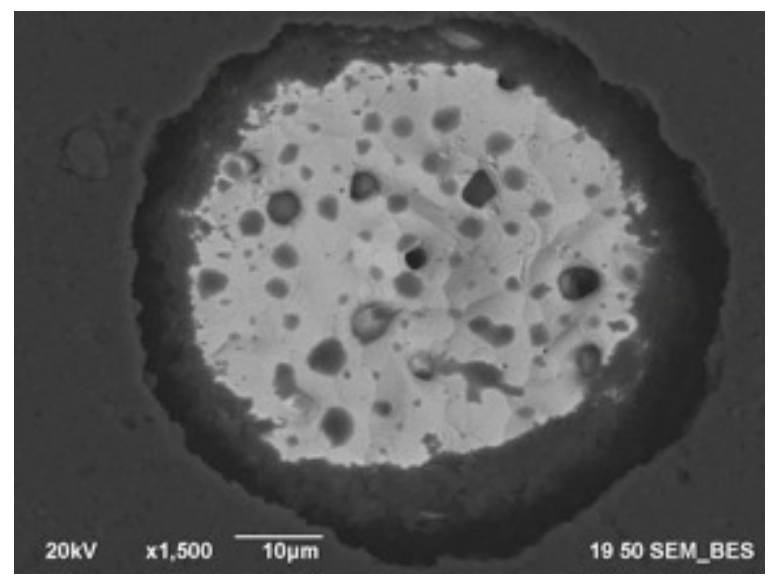

Backscatter

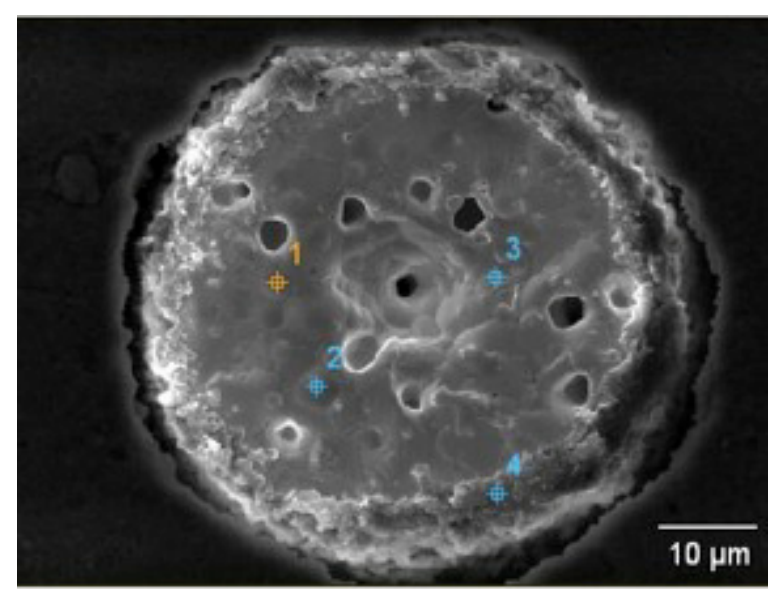

Secondary Electron

Figure 6-12 SEM image of fired feedthrough in a air atmosphere at $1550{ }^{\circ} \mathrm{C}$

Point 3 in figure 6-12 shows the average composition of the via. It is mostly occupied with platinum and has aluminum, carbon and oxygen as impurity. Presence of carbon could be attributed to an incomplete burn-out process. Aluminum and oxygen could be from the diffusion of alumina in the platinum in high temperature. However, the presence of all three elements could also be due environmental contamination and experimental error.

The elemental analyses of point 1 and 2 indicated the presence of $\mathrm{Si}, \mathrm{Mg}$ and $\mathrm{Ca}$ in addition to $\mathrm{Pt}, \mathrm{Al}, \mathrm{C}$ and $\mathrm{O}$. This elemental composition implies the formation of the glass on the surface of platinum via. Glass particles usually have the flake-like structure with the average size of 10-20 $\mu \mathrm{m}$, but the glass particles shown in figure 6-12 have spherical shape with size of 5 $\mu \mathrm{m}$. This indicates the melting of glass at high temperatures. At high temperatures, melted glass failed to wet the platinum and floated to the surface of the via, and solidified into the spherical shape in the cooling process. In the cool down process, the temperature of the surface of platinum is less than the bulk. Based on figure 1-9, this temperature gradient could differentiate the glass/metal surface tension and produces capillary force to push the molten glass out of the metal. This mechanism can explain the formation of glass on the top surface of platinum via. 

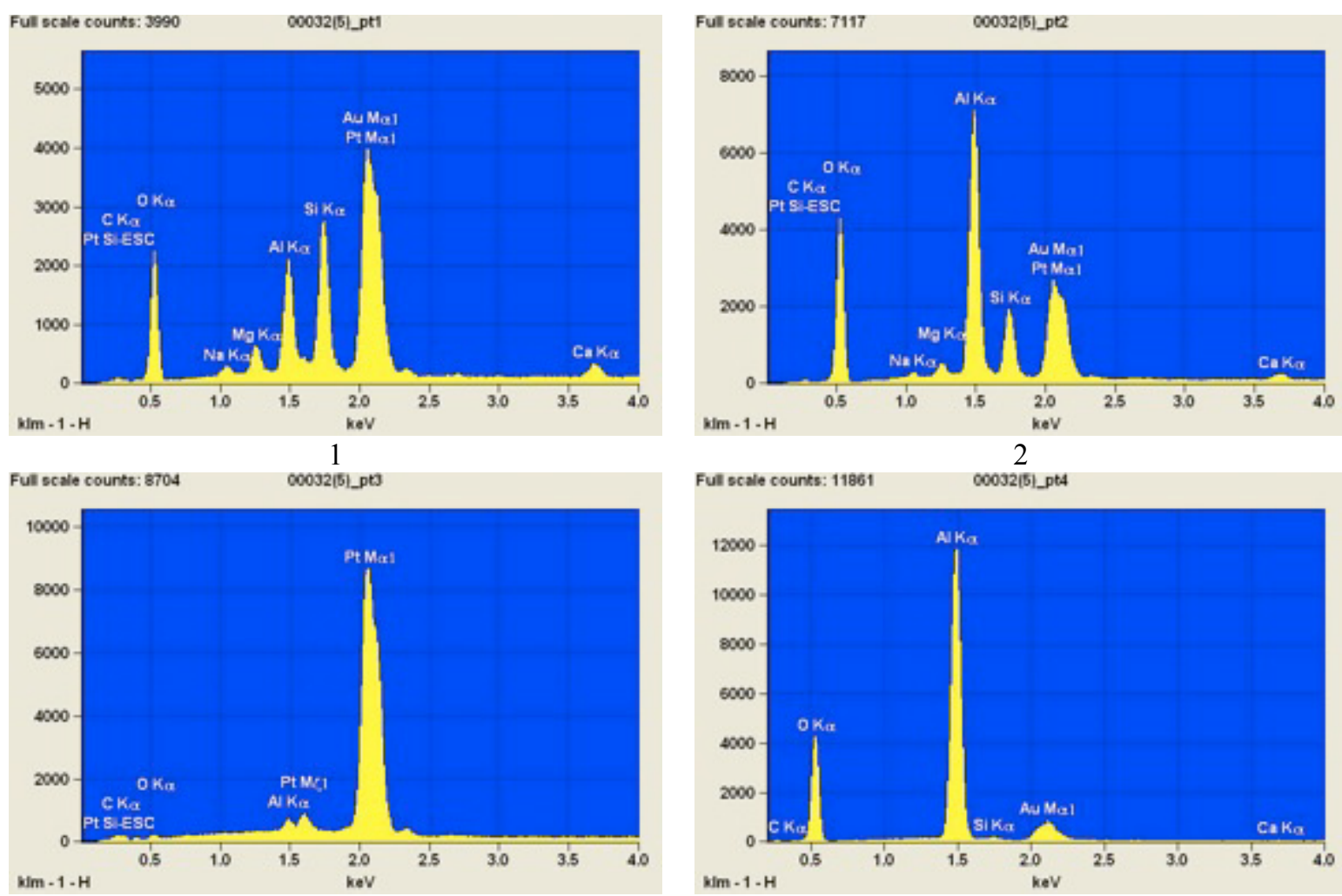

3

4

Figure 6-13 EDS analyses of the different point in the platinum via from figure 6-13

Another fact that could support the proposed glass migration mechanism is the elemental composition of the ceramic structure around platinum. The adhering of ceramic to the sidewalls of platinum was shown before in figures 6-3. That bonding could be completely physical in nature and due to the migration of glass. However elemental analyses of point 4 shows mostly Al and $\mathrm{O}$, which could rule out the effect of glass on the bonding formation of platinum/alumina and suggests the migration of glass to the surface of via.

The EDS analyses of the cross section of the platinum via is shown in figure 6-14. It could be seen that there is almost no trace of glass component in the platinum via. As showed previously, glass melted and moved to the top surface of the via. 


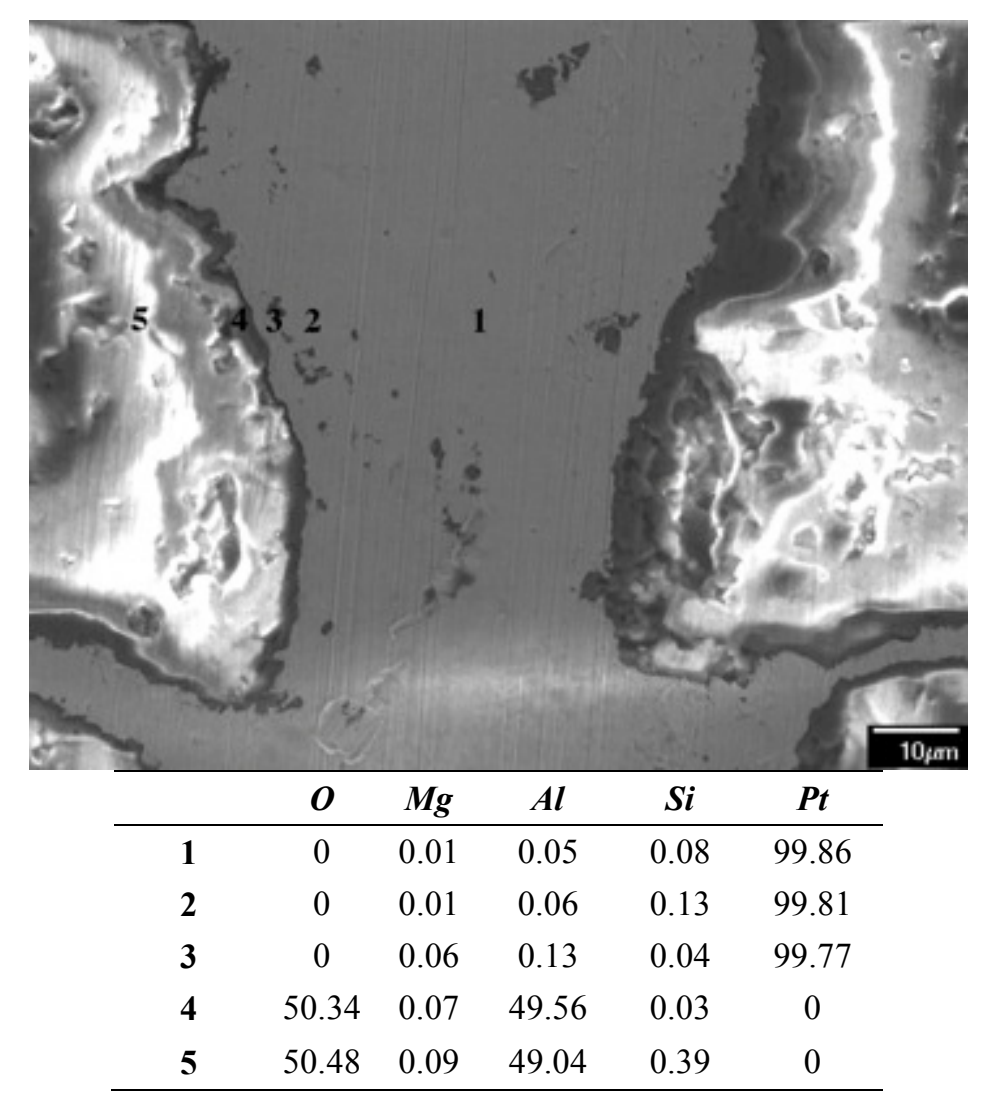

Figure 6-14 EDS analyses of the cross section of the platinum via fired in the air atmosphere

\subsection{Sample annealing}

To study the effect of heat treatment on the metal/ceramic bonding, an already fired feedthrough was heated up to $1550{ }^{\circ} \mathrm{C}$ and then kept on that temperature for 5 hours. The results of the annealing process are shown in figure 6-15.

As it can be seen, the annealing process really did not change the range of metal/ceramic bonding. However, it created a large crack in the middle of platinum via. The internal side of the crack, which is shown in high magnification image, is completely sharp. This indicates the formation of the crack at high temperatures. At high temperature the shrinkage of platinum is greater than alumina. The solid platinum is a material, so if the bonding of platinum to the surrounding ceramic is strong enough, platinum could fracture instead of delamination from the 
ceramic wall. After the crack generation in high temperature, atoms can move and create a clean and sharp surface at high temperatures.
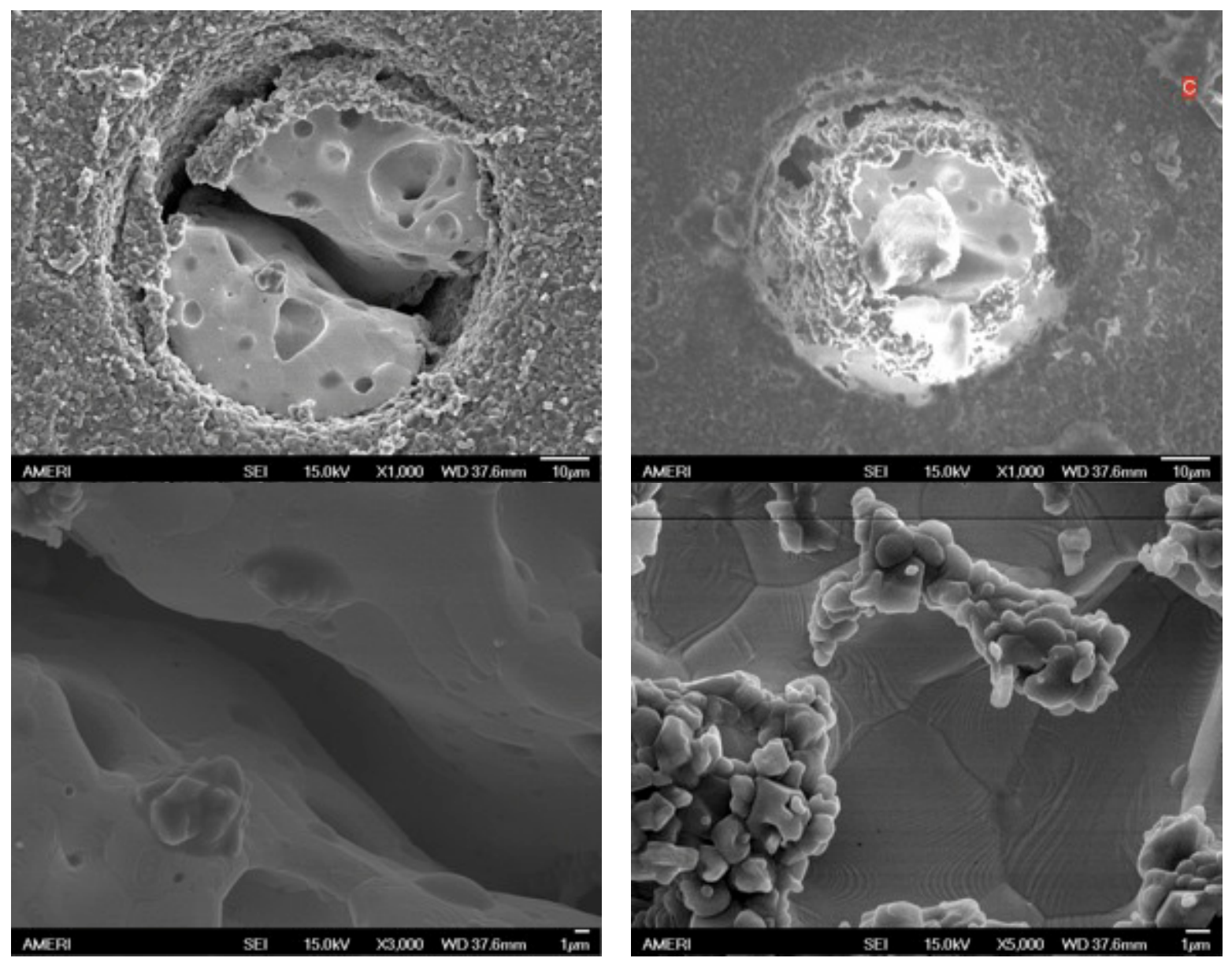

Figure 6-15 SEM image of annealed sample for $5 \mathrm{hr}$ at $1550{ }^{\circ} \mathrm{C}$

The grain size of platinum is same as what observed in figure 6-3 (around $5 \mu \mathrm{m}$ ). This means that the heat treatment process does not have any affect on the grain growth of platinum particles. 


\subsection{Platinum metallization}

\subsubsection{Platinum group metallization}

As described earlier, the platinum group metals (i.e. iridium, ruthenium and rhodium) have been used as the alloying elements to improve the platinum/alumina bonding strength. To study the effect of the introduction this kind of metallization, two platinum inks were prepared with $20 \% \mathrm{Wt}$. concentration of iridium and rhodium. The inks were used to prepare a ceramic feedthrough and the results of the firing of such feedthroughs are shown in figure 6-16.

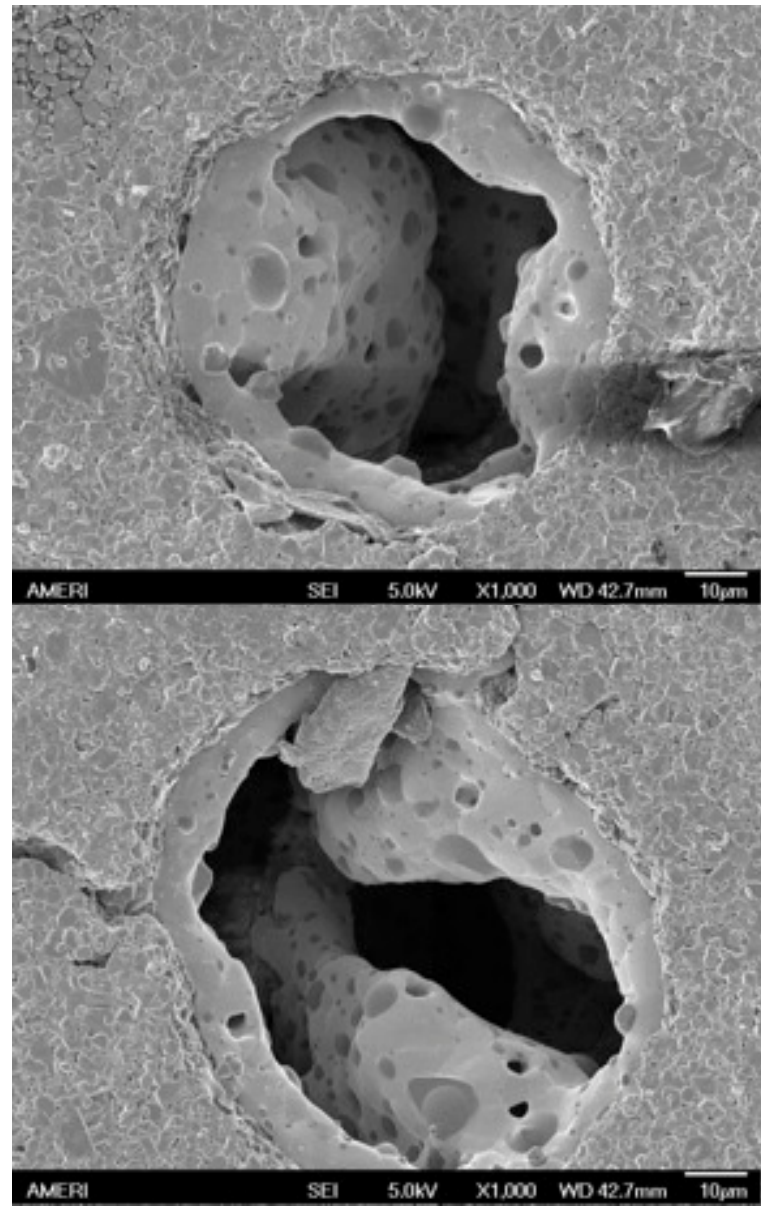

Pt-4 + 20\% Iridium

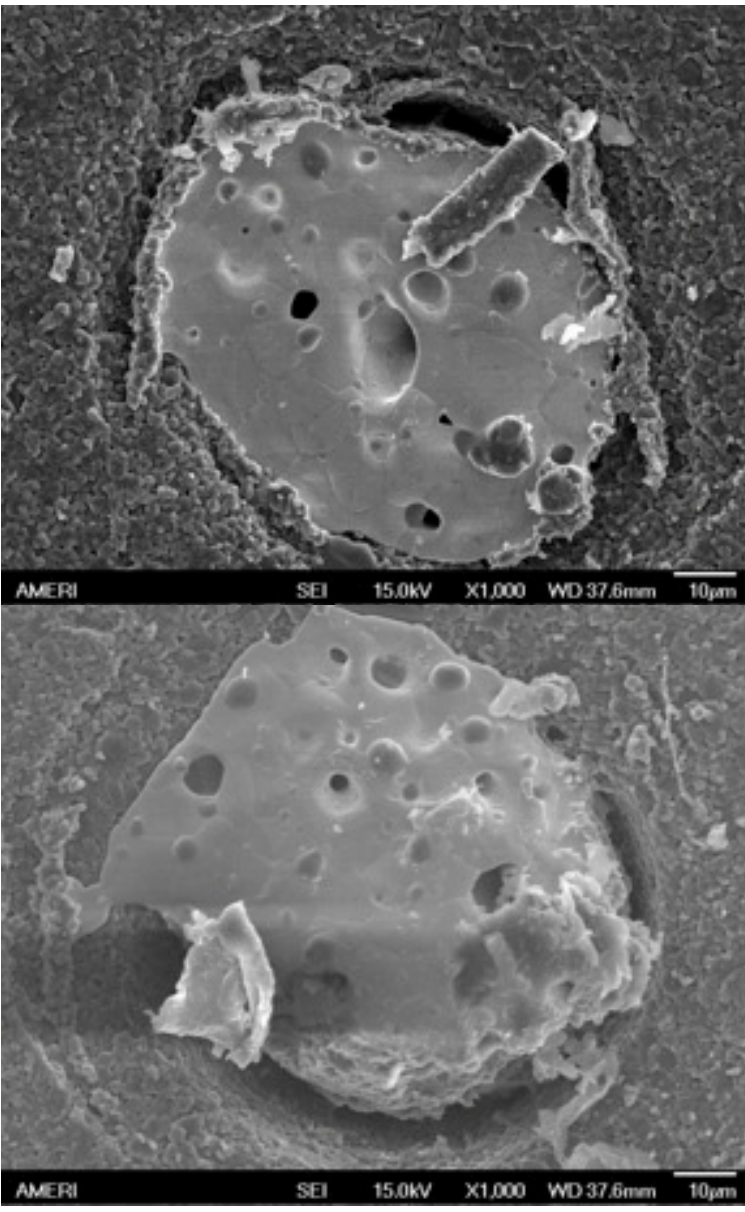

Pt-4 + 20\% Rhodium

Figure 6-16 Electron microspoe image of fired feedthrough with different metalization

Iridium and rhodium can create stable oxides below $1000^{\circ} \mathrm{C}$ and this oxide can increase

the adhesion between platinum and alumina. However, the weight loss experiments from figure 
5-9 showed that iridium is mostly volatile after $1100^{\circ} \mathrm{C}$ due to the formation of the gaseous species of iridium oxide. Rhodium also showed the same kind of behavior yet the extent of vaporization of rhodium is less than iridium. In addition to that, the mixture of Pt-4+20\% $\mathrm{Ir}$ almost showed the same behavior as pure iridium. This suggests the catalytic effect of iridium on the vaporization of platinum. As shown figure 6-16, the iridium metallized sample is almost empty and all the material escaped form the via.

The combination of rhodium with platinum can work better than iridium. The Pt-4 + $20 \% \mathrm{Rh}$ sample also showed the delamination of the metal from alumina like the pure platinum sample. Thermal expansion and densification rate of rhodium is less than platinum. Because of that, it is expected that the introduction of rhodium could decrease the shrinkage of platinum. Yet, the shrinkage is not enough to make a strong bond between platinum and alumina.

Table 6-2 Melting temperature and thermal expansion of different metals and oxide

\begin{tabular}{lcc}
\hline & Melting temperature $\left({ }^{\circ} \mathbf{C}\right)$ & Thermal expansion $(\mu \mathrm{m} /(\mathrm{m} \cdot \mathrm{K}))$ \\
\hline $\mathrm{Pt}$ & 1768 & 8.8 \\
$\mathrm{Ir}$ & 2739 & 6.4 \\
$\mathrm{Rh}$ & 2237 & 8.2 \\
$\mathrm{~W}$ & 3695 & 4.5 \\
$\mathrm{Mo}$ & 2896 & 4.8 \\
$\mathrm{~A} 2 \mathrm{O} 3$ & 2072 & 5.4 \\
Y2O3 & 2425 & 8.1 \\
WO3 & 1473 & 13 \\
$96 \%$ Alumina tape & -- & 6 \\
\hline
\end{tabular}

Refractory metals like tungsten could also be used as the metallization in ink formulation. High melting point and low thermal expansion makes tungsten an ideal elect for this purpose, table 6-2 shows the thermal expansion values of different metals and oxides. However, refractory metals create volatile oxide above $1000^{\circ} \mathrm{C}$. The level of evaporation of tungsten and other refractory metals are much higher than iridium. Hence, their application in air is impossible and 
suggested processing in a reduced atmosphere. The effect of tungsten metallization was studied thoroughly in this project and will be presented in the next chapter.

\subsubsection{Oxide metallization}

Oxides are mostly stable materials and their thermal expansion and shrinkage is less than the platinum powders. Because of that, the introduction of an oxide to the ink formulation is another way to decrease the platinum's shrinkage with minimal impact on electrical conductivity. Alumina is the first choice because it is same as the ceramic tape material. Two different compositions of alumina (i.e. $20 \%$ Vol. and $20 \% \mathrm{Wt}$.) were used to develop platinum ink. The resultant feedthroughs are shown in figure 6-17.

It was expected that mixture of platinum and oxide could create an aggregate composite to control the shrinkage. However, composites need an excellent dispersion to achieve the best performance. Despite the concentration of the alumina powder in the ink formulation, a layer of alumina was formed on the top surface of all vias. As described before in the section 2.2.2, glass flows to the top surface of the platinum due to the capillary force. The same phenomena could happen to alumina as well. Specific density of alumina is approximately 0.1 of the specific density of platinum. Because of that, the slightest capillary force could push the alumina to the surface. It is clear from the figure 6-17 that the sample with $20 \%$ wt. alumina $(\sim 50 \%$ Vol.) shows the migration of the alumina powder. On the other hand, it shrinks less than the other sample due to higher concentration of alumina. The sample with $20 \%$ Vol alumina still shows delamination from the ceramic and suggests that alumina is not a good oxide selection for platinum metallization to control the sintering and densification of platinum. 

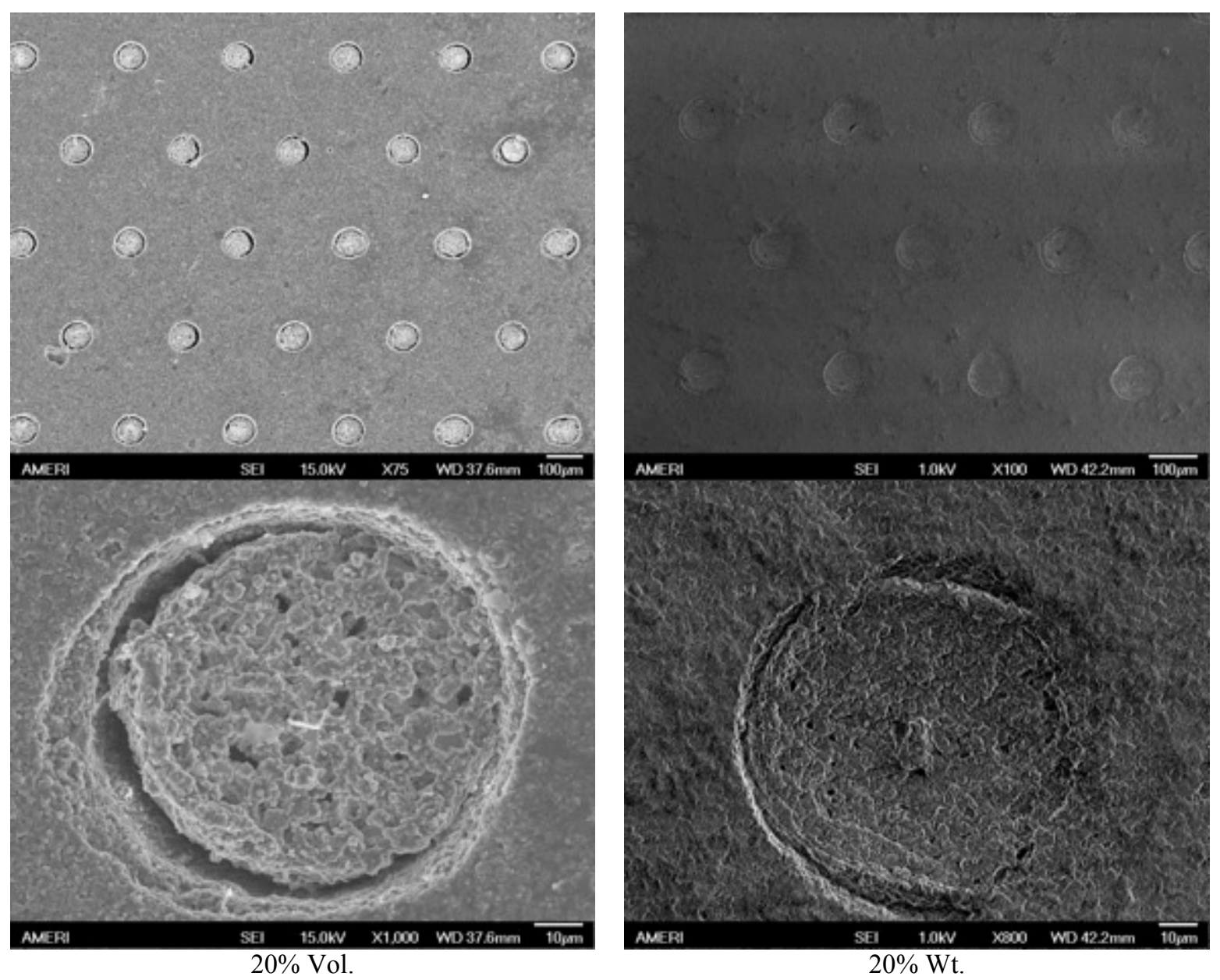

Figure 6-17 SEM image of ceramic feedthrough metalized with alumina

Powel [140] showed that yttrium oxide could react with platinum and control the sintering and shrinkage of platinum Because of that, conductive platinum ink with $20 \% \mathrm{Vol} \mathrm{Y}_{2} \mathrm{O}_{3}$ was prepared to study the effect of yttrium oxide on sintering of platinum. The result of such feedthrough is shown in figure 6-18.

The introduction of yttrium oxide could not inhibit the sintering and densification of platinum and the via material pulled back from the walls. Similar to the image $6-17$, the surface of the platinum is covered with the oxide, but the shrinkage of platinum with $\mathrm{Y}_{2} \mathrm{O}_{3}$ is more than platinum with $\mathrm{Al}_{2} \mathrm{O}_{3}$. It is clear from the EDS mapping that the surface of the via is almost cover with yttrium oxide. 

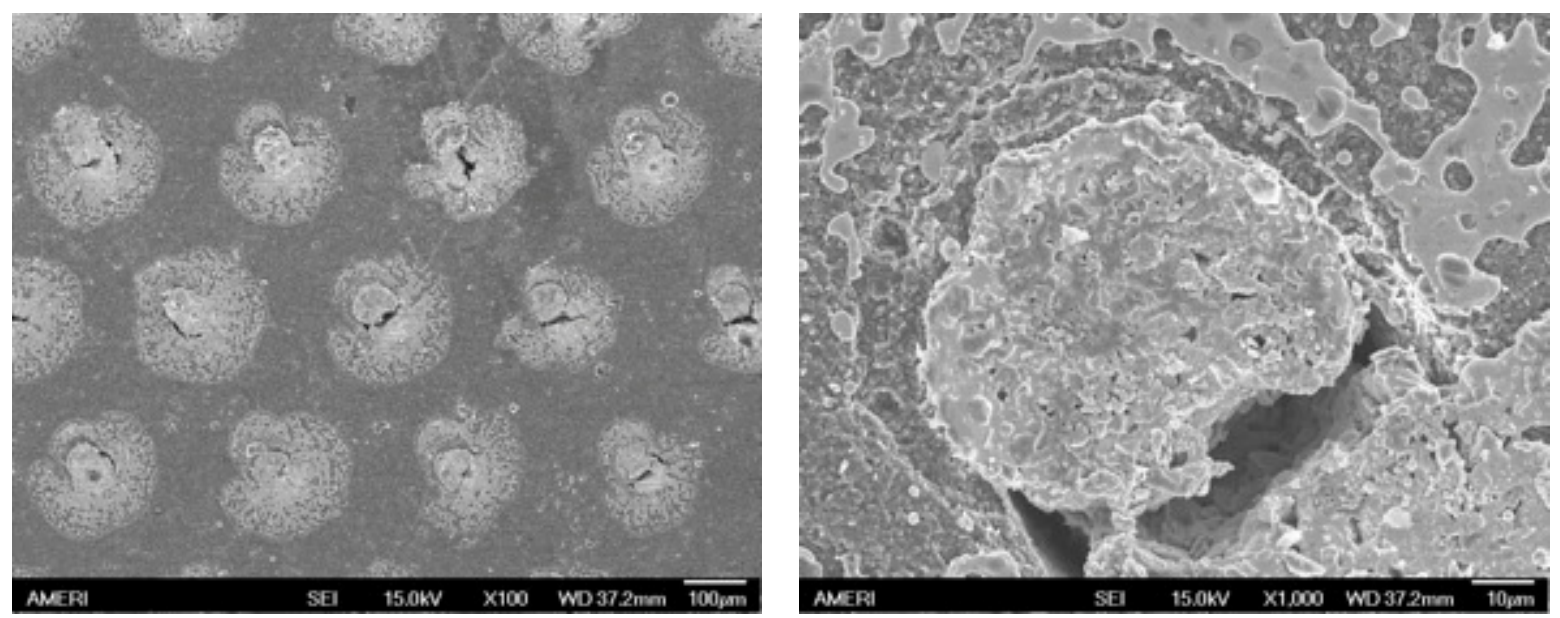

Figure 6-18 SEM image of ceramic feedthrough metalized with ytreium oxide
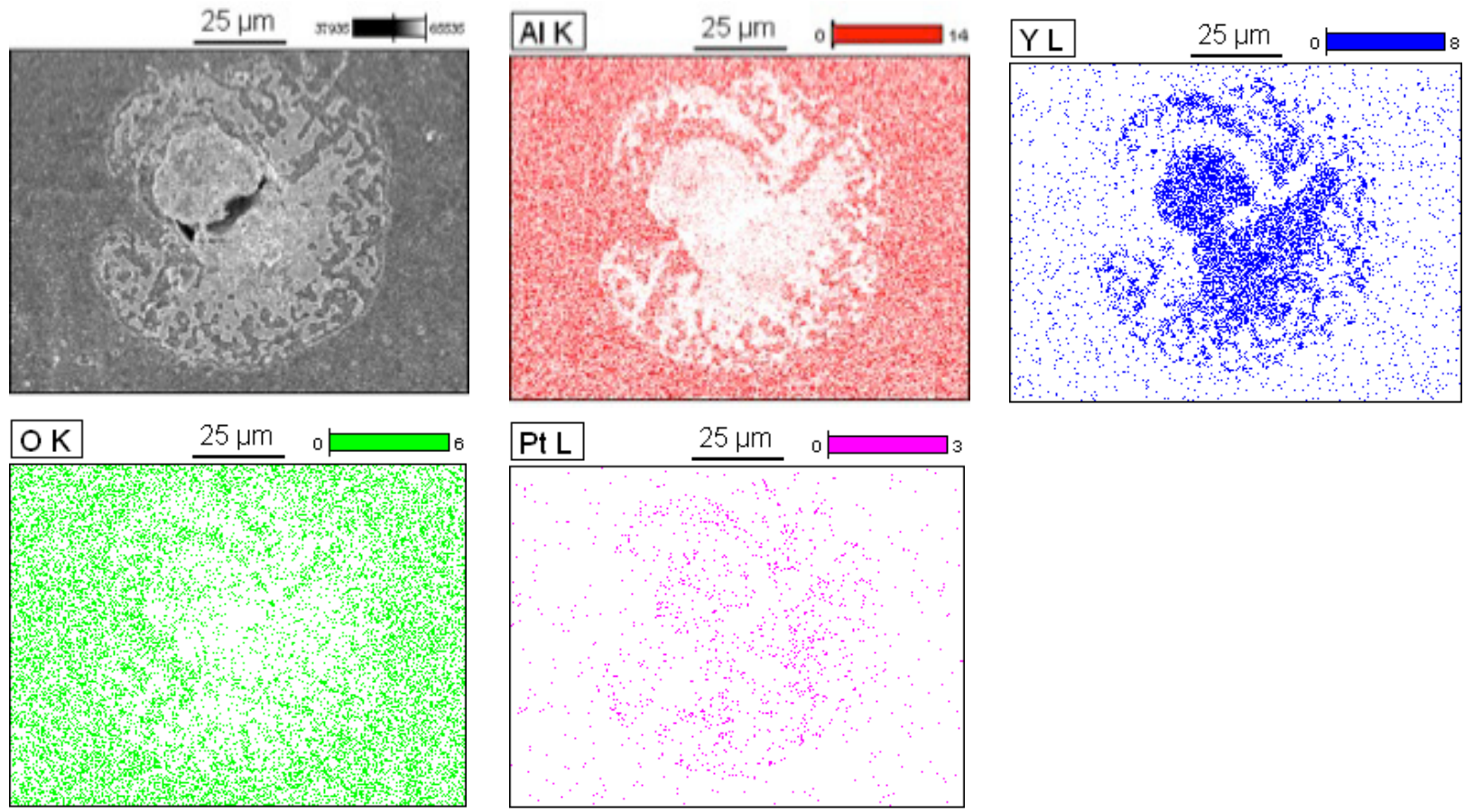

Figure 6-19 EDS mapping of the of ceramic feedthrough metalized with ytreium oxide

It could be concluded that introduction of oxides in air atmosphere is not an appropriate way to control the shrinkage of the platinum powder. 


\section{Feedthrough development in reduced atmosphere}

\subsection{Introduction}

Bonding and reaction of platinum and alumina could be enhanced in reduced atmosphere $[6,55]$. Hydrogen can reduce alumina and different reaction products are reported from this reaction based on the concentration of hydrogen and temperature of the reaction. Pure hydrogen atmosphere can only worsen the shrinkage situation by increasing the reduction reaction of platinum and increasing the shrinkage of platinum [129]. In addition, hydrogen could soften the Pt-Pt bond and decreases the melting the temperature of platinum [147]. The results of a sample feedthrough fired in two different concentrations of hydrogen at $1550{ }^{\circ} \mathrm{C}$ are shown in figure $7-1$.

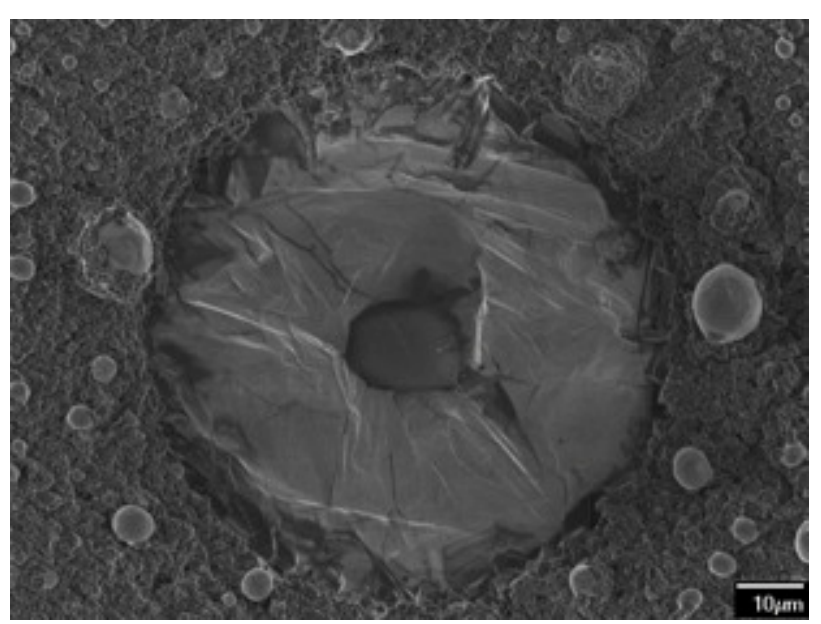

$\mathrm{H}_{2}-\mathrm{Ar}(10-90)$

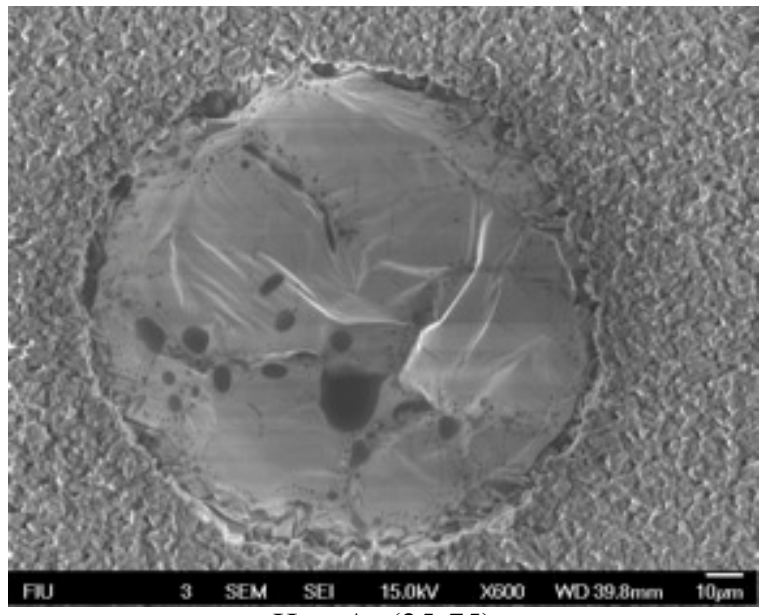

$\mathrm{H}_{2}-\operatorname{Ar}(25-75)$

Figure 7-1 SEM image of feedthrough fired in two different concentration of hydrogen

It is clear from figure 7-1, the platinum powder was melted and forms a very dense structure. Backscatter image shown in figure 7-2 and EDS elemental analyses shown in figure 73 indicated that alumina and glass particles melted and came to the surface of the via. Ceramic has reinforced the glass phase to the sidewalls of the via and created a dense structure around the 
via. A large particle of the ceramic is also showed in the surface of the platinum via. Alumina melted completely and moved to the surface of the platinum.

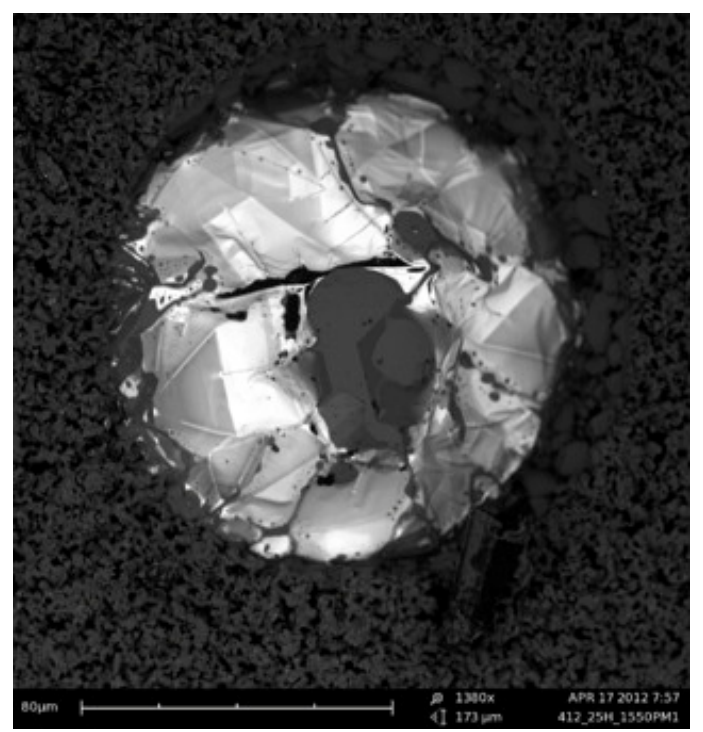

Figure 7-2 Backscatter SEM image of a sample platinum via fired in $\mathrm{H}_{2}-\mathrm{Ar}(25-75)$ atmosphere at $1550{ }^{\circ} \mathrm{C}$ The major problem of the firing in reduced atmosphere was observed with samples fired in two different furnaces. A small self-fabricated furnace was used to study the effect of temperature, hydrogen concentration and atmospheric humidity. These parameters allowed us to optimize the firing profile parameters. However, when a sample was fired in the commercial Camco G-1600 under the same condition, the density and shrinkage of the samples were significantly different. A ceramic firing ring from Ferro Co. was used to analyze the temperature and heat absorption in both furnaces. The diameter of the ring depends on the final temperature and the total heat absorbed, which makes it possible to measure the relative performance in each furnace. The results showed that the temperature control is similar in both furnaces; these differences can be due to the different in heat convection mechanism in different furnaces. Platinum particle size and platinum metallizations were evaluated to develop a hermetic feedthrough. 


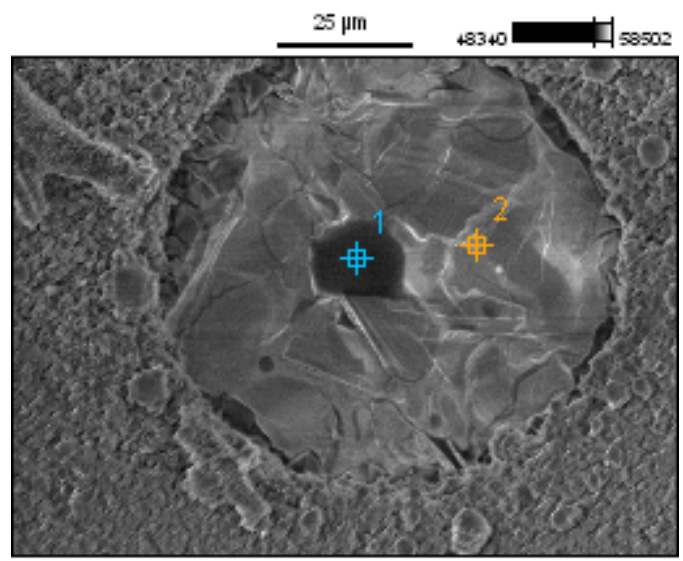

\begin{tabular}{cccccc} 
& $\boldsymbol{O}-\boldsymbol{K}$ & $\boldsymbol{A l}-\boldsymbol{K}$ & $\boldsymbol{S i}-\boldsymbol{K}$ & $\boldsymbol{M g}-\boldsymbol{K}$ & $\boldsymbol{P t}-\boldsymbol{L}$ \\
\hline Point1 & 40.93 & 17.36 & 20.27 & 21.44 & 0.00 \\
Point & 2.14 & 1.51 & 6.49 & 0.13 & 89.74 \\
\hline
\end{tabular}

Figure 7-3 EDS elemntal analyses of a sample platinum via fired in $\mathrm{H}_{2}-\mathrm{Ar}(10-90)$ atmosphere at $1550{ }^{\circ} \mathrm{C}$

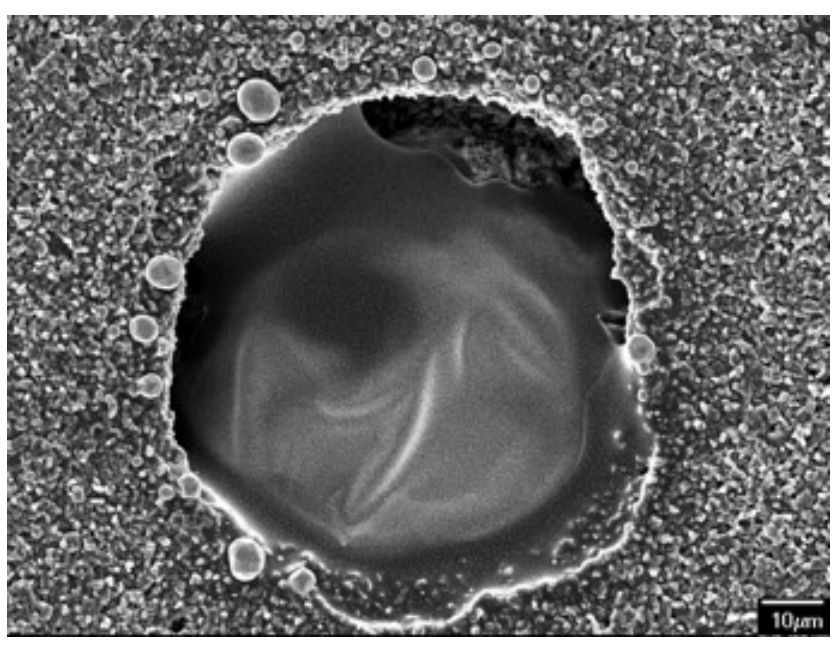

Figure 7-4 SEM image of two samples via. Feedthrough fired in in H2-Ar (10-90) atmosphere in Camco G1600 at $1550{ }^{\circ} \mathrm{C}$

\subsection{Firing temperature}

Temperature is one of the most important parameters that controls the final microstructure of the feedthrough sample. Because of that, the effect of firing temperature on the properties of the feedthrough in a reduced atmosphere were studied and are given in figures 7-5 and 7-6. Pt-4 and Pt-5 platinum particles were used to develop the feedthrough. After the burn-out process, each feedthrough was heated up to the desired temperature at the heating rate of $10^{\circ} \mathrm{C} / \mathrm{min}$, maintained at that temperature for an hour and was rapidly cooled down. Firing was done in the $\mathrm{H}_{2}-\mathrm{Ar}(25-75)$ atmosphere. 

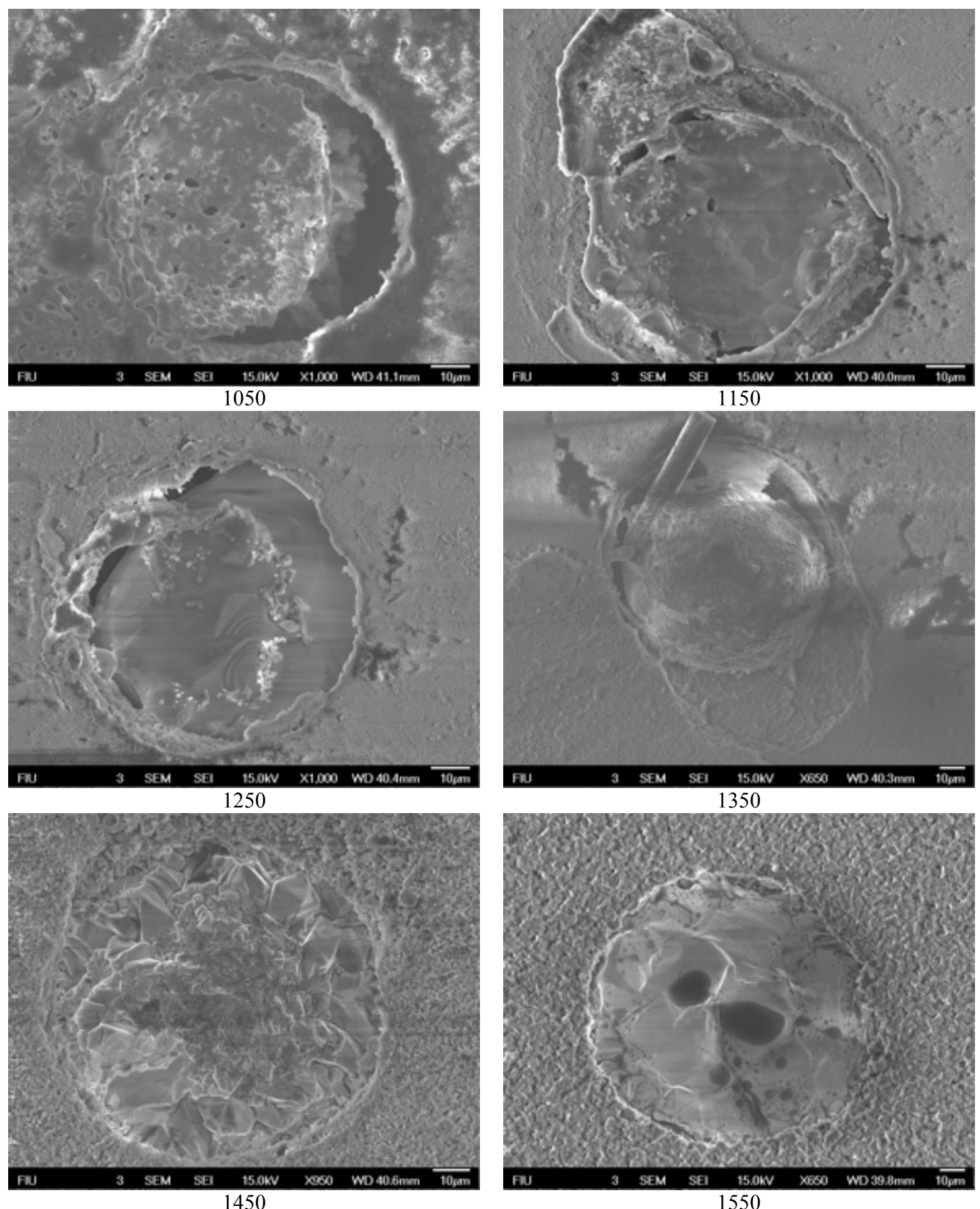

Figure 7-5 SEM image of the Pt-4 platinum powder via fired at different temperatures. Feedthroughs are fired in the $\mathrm{H}_{2}-\mathrm{Ar}$ (25-75) atmosphere 

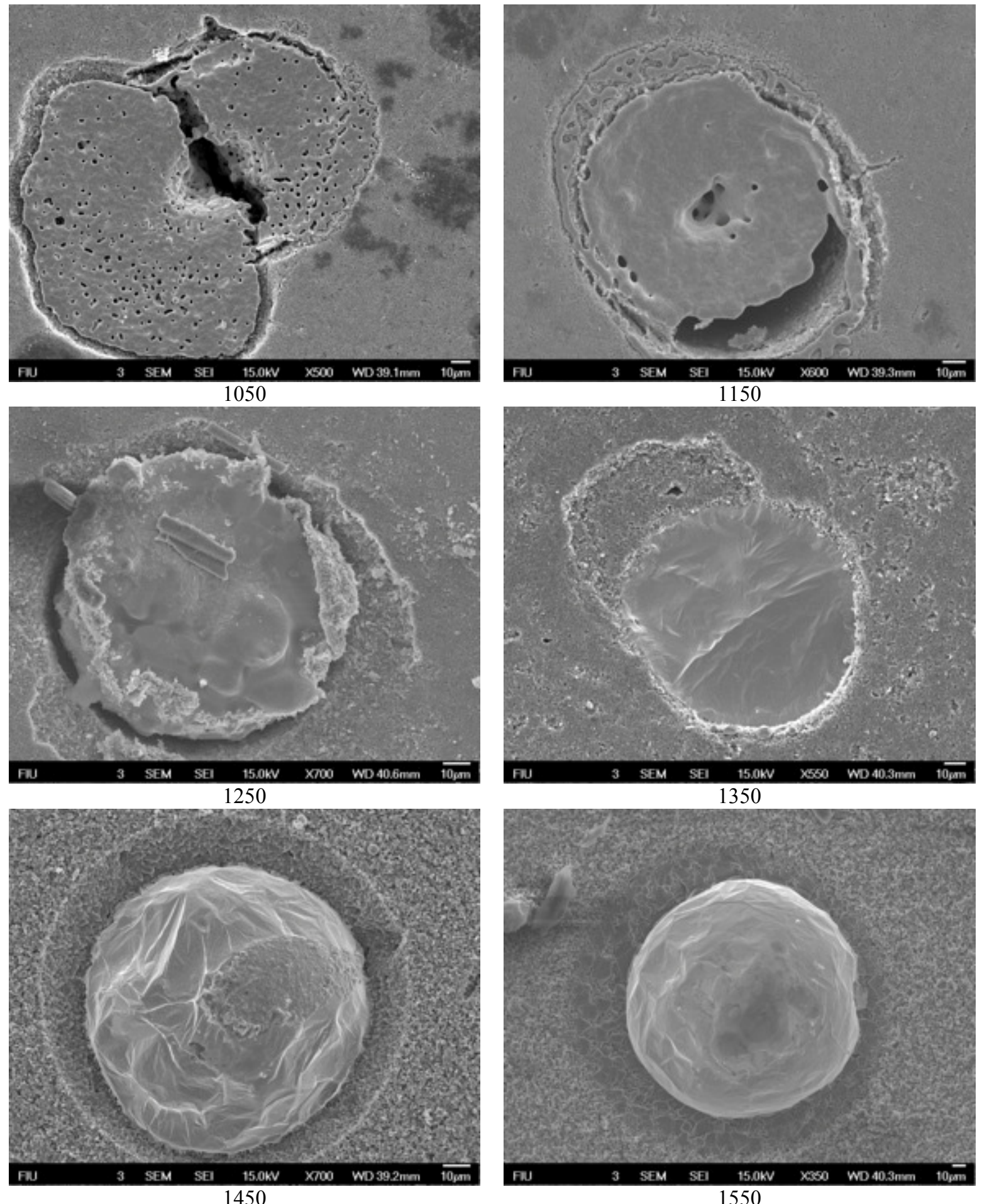

Figure 7-6 SEM image of the Pt-5 platinum powder via fired at different temperature. Feedthroughs are fired in the $\mathrm{H}_{2}-\mathrm{Ar}(25-75)$ atmosphere

In the lowest firing temperature (i.e. $1050^{\circ} \mathrm{C}$ ), both particles start to densify. Pt-4 contracts and shrinks, while Pt-5 expands and covers the surrounding area. In the temperature 
range of $1150-1250^{\circ} \mathrm{C}$, both particles follow almost the same trend and start to contract similarly. The temperature is not high enough to exhibit a reaction between the ceramic and metal. There is ceramic debris structure around Pt- 4 at $1150{ }^{\circ} \mathrm{C}$ and at $1250{ }^{\circ} \mathrm{C}$ around Pt-5, which is very similar to what was observed before in feedthroughs fired in an air atmosphere.

The behavior of platinum powders starts to change after the temperature reaches $1350{ }^{\circ} \mathrm{C}$. In the range of $1350-1550{ }^{\circ} \mathrm{C}, \mathrm{Pt}-5$ starts to expand and fill the via. By increasing the temperature, platinum starts to expand and ultimately makes a dome-like structure above the via. At $1350^{\circ} \mathrm{C}, 1450^{\circ} \mathrm{C}$ and $1550^{\circ} \mathrm{C}$, the platinum via is $97 \mu \mathrm{m}, 105 \mu \mathrm{m}$ and $150 \mu \mathrm{m}$ in diameter, respectively. At $1350{ }^{\circ} \mathrm{C}$, platinum covers the surface, similar to $1050{ }^{\circ} \mathrm{C}$ and covers the larger area. Later, it starts to contract and fill the via. As is seen from figure $7-5$, at $1450^{\circ} \mathrm{C}$, the ceramic around the platinum disappears and creates a recessed structure. This behavior extends at $1550{ }^{\circ} \mathrm{C}$ and the recessed area around the platinum increases. At $1450{ }^{\circ} \mathrm{C}$, the recessed area is $135 \mu \mathrm{m}$ in diameter and at $1550{ }^{\circ} \mathrm{C}$, it is about $200 \mu \mathrm{m}$. At temperatures above $1450{ }^{\circ} \mathrm{C}$, platinum starts to dissolves alumina from the surrounding area and expands. By the introduction of alumina and glass particles into platinum, platinum starts to expand up to $150 \mu \mathrm{m}$ and creates a dome structure.

The interaction of the Pt-4 particle and alumina is more complicated under the same conditions. At $1350^{\circ} \mathrm{C}$, platinum densifies more and forms a complete sphere. By increasing the temperature, platinum starts to expand and fill the via. In temperature range of $1350-1450{ }^{\circ} \mathrm{C}$, a coating of alumina covers the surface. In this temperature range, platinum dissolves some of the ceramic from the sidewalls and pushes them to the top surface. At $1350^{\circ} \mathrm{C}$, the alumina coating has a small grain size and does not cover the whole surface. However, at $1450^{\circ} \mathrm{C}$, the alumina covers the surface completely with a large grain structure. As is shown in figure 7-6, it is also 
obvious that a small portion of the platinum via at $1450^{\circ} \mathrm{C}$ is covered with alumina. Platinum powder dissolves alumina at high temperatures, however, different final microstructure of the two platinum particles at $1450{ }^{\circ} \mathrm{C}$ could suggest two different reactions:

1. Smaller size particles wick the alumina and push it to the top. This can be due to the higher density of smaller particles at high temperatures.

2. High surface area of the smaller particle increases the interaction between platinum and alumina and increases the amount of solute.

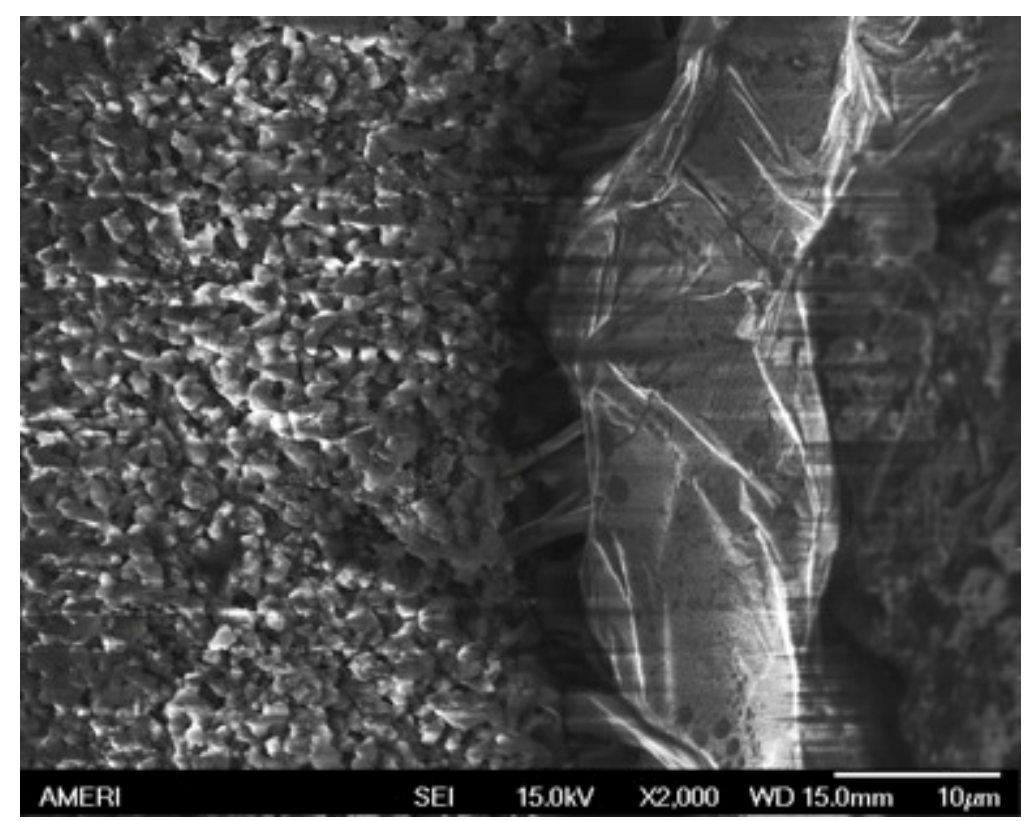

Figure 7-7 SEM image of Pt-4/alumina boundary area. Feedthrough fired in the $\mathrm{H}_{2}-\mathrm{Ar}(25-75)$ atmosphere at $1550{ }^{\circ} \mathrm{C}$

Due to grains growth of platinum particles at high temperatures, the first reaction is most probable. At the highest temperature, platinum completely dissolves the ceramic and there is no sign of it on the surface. As is shown in the backscatter image of the Pt-4 sample in figure 7-2, ceramic covers around the via and strongly bonds with it. A large droplet shape ceramic also appears on top of the platinum via, but it does not have a grain structure. The ceramic structure is more like a solidified glass, which was analyzed in figure 7-3. This behavior could suggest the 
complete solution of alumina and glass in platinum at high temperatures. Figure 7-8 shown the concentration gradient of different elements in platinum for the Pt-5 platinum powder via fired at $1550{ }^{\circ} \mathrm{C}$, detremiuned by EDS analyses.

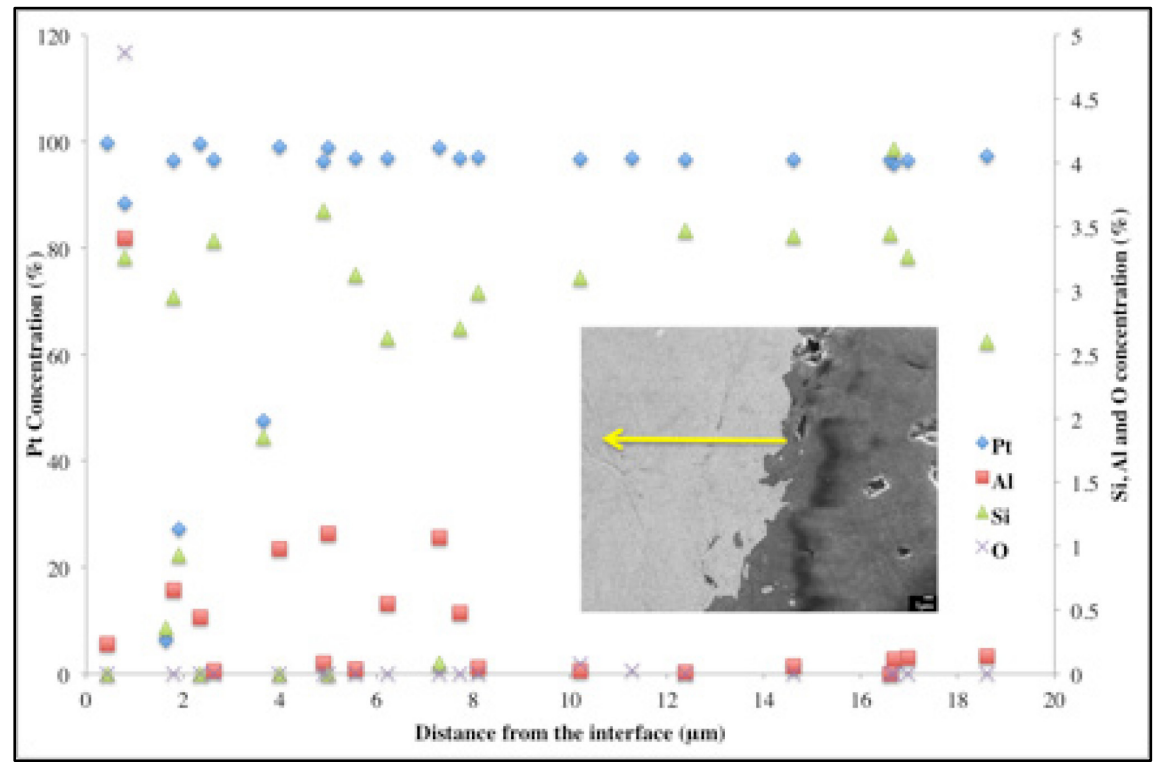

Figure 7-8 Concentration gradient of different element in platinum for the Pt-5 platinum powder. Feedthrough fired in the $\mathrm{H}_{2}-\mathrm{Ar}(25-75)$ atmosphere at $1550{ }^{\circ} \mathrm{C}$

It is obvious that silicon diffuse majorly through the entire width of the via. The weight concentration of the silicon is almost constant and is about 3.5\%. Pt-Si phase diagram [148] does not show any solid solution of silicon of silicon in platinum. Therefore, the $3.5 \%$ concentration of silicon in platinum suggests the reaction of $\mathrm{SiO}_{2}$ with platinum and the creation of a $\mathrm{Pt}_{3} \mathrm{Si}$ phase. Silicate compounds, which are used mostly as the glass phase in HTCC ceramic tape formulation, could be the source of $\mathrm{SiO}_{2}$ for reaction. Aluminum is mostly availably in the first few microns from the interface. The concentration of aluminum in platinum is about 1.5 Wt.\%, which is equal to the solubility of aluminum into platinum [149]. After about 8 microns form the interface, the concentration of the alumina dropped drastically and it is almost negligible. This behavior could suggest that silicon prevents diffusion of aluminum into platinum. 
To minimize the effect of silicon on the diffusion of the aluminum, the $99.9 \%$ HTCC ceramic tape were use to develop a feedthrough and the concentration gradient of different elements are shown in figure 7-9. It is clear that the concentration of the aluminum gradually decrease, however the final concentration is about $5 \mathrm{Wt} \%$, which is more than the solubility limit of the aluminum in platinum. This behavior promotes the formation of $\mathrm{Pt}_{3} \mathrm{Al}$ phase. The concentration of oxygen shown in Figure 7-8 and 7-9 is almost zero in all cases, which is due to the reduction of alumina and silica in the reduced atmosphere. It should be noted that EDS does not show any trace of platinum in alumina, which indicates that diffusion only happened from the alumina side to the platinum.

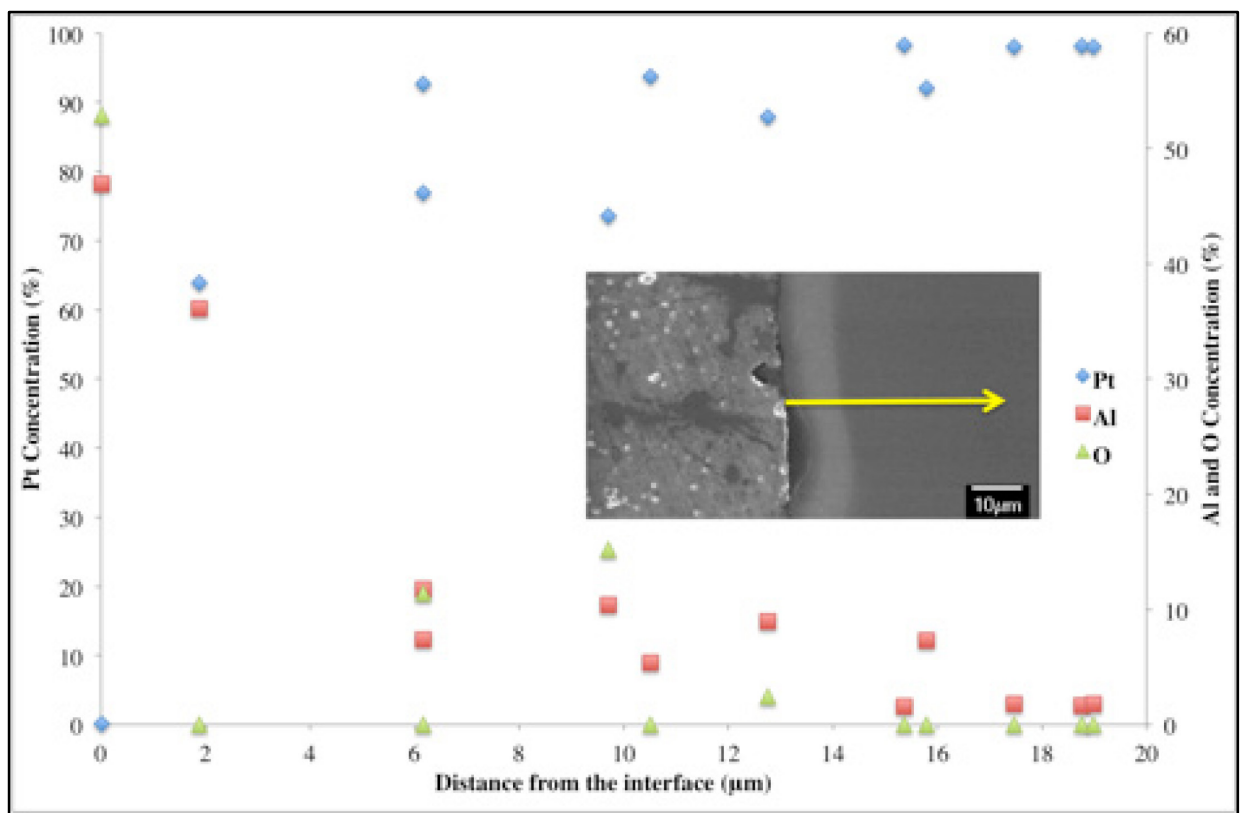

Figure 7-9 Concentration gradient of different element in platinum for the Pt-5 platinum powder and 99.9\% HTCC ceramic tape. Feedthrough fired in the $\mathrm{H}_{2}-\mathrm{Ar}(25-75)$ atmosphere at $1550{ }^{\circ} \mathrm{C}$

At high temperatures, platinum absorbs the ceramic grains and glass from the sidewalls and that expands the structure. Platinum dissolves silicon and cannot maintain the solubility of the aluminum, thus the aluminum moves comes to the surface. Based on the temperature, alumina could recrystallize to large grains or small grain size. However, at temperatures above 
$1500^{\circ} \mathrm{C}$, it dissolves into the platinum and remains as a reaction product. This could be due to the formation of a Pt-Al compound as a second phase in the platinum-alumina interface.

The cross section SEM images of Pt-4 and Pt-5 samples fired in $1550^{\circ} \mathrm{C}$ are shown in figure 7-10. Both samples showed a very dense structure. The via in each layer diffused completely to each other, which provides continues connectivity through the feedthrough.

However, in some cases high z-axis shrinkage of the ink broke the continuity of the platinum via. It is also clear that there is a significant boundary line between metal and ceramic. This clear boundary region makes it difficult to characterize the diffusion of alumina in platinum.

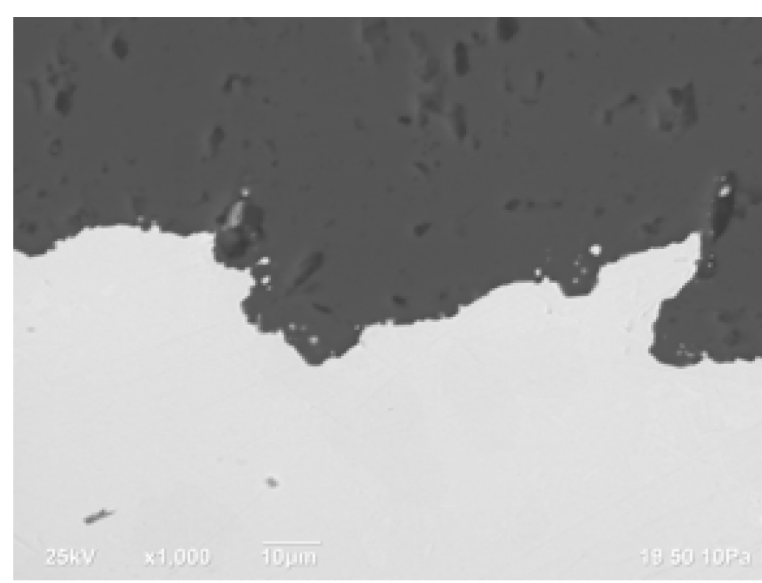

$\mathrm{Pt}-4$

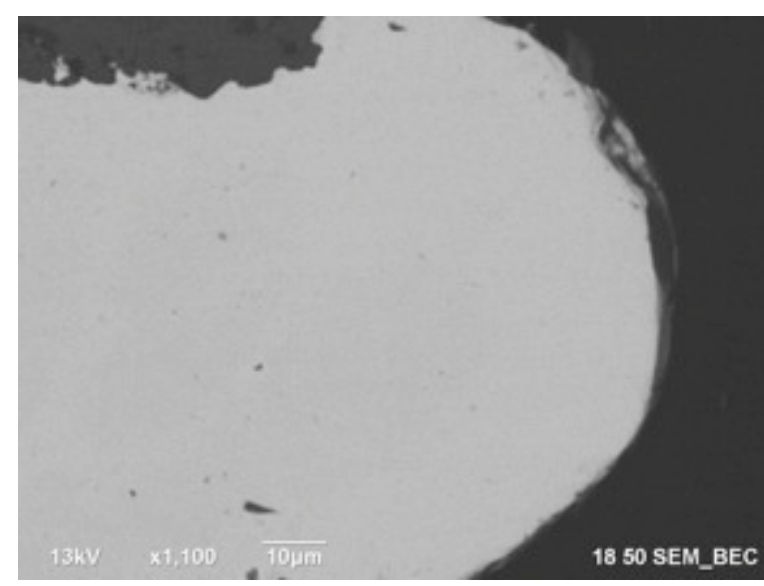

Pt-5

Figure 7-10 Backscatter image of the platinum via from two different particles. Feedthroughs are fired in the $\mathrm{H}_{2}-\mathrm{Ar}(25-75)$ atmosphere at $1550^{\circ} \mathrm{C}$

\subsection{Hydrogen concentration}

As was indicated before, in the temperature range of $1350-1450{ }^{\circ} \mathrm{C}$, the surface of the platinum is covered with alumina. A series of feedthrough samples with Pt-4 platinum particles were fired at $1400{ }^{\circ} \mathrm{C}$ in different hydrogen concentrations. The SEM images of these samples are shown in figure 7-11. 


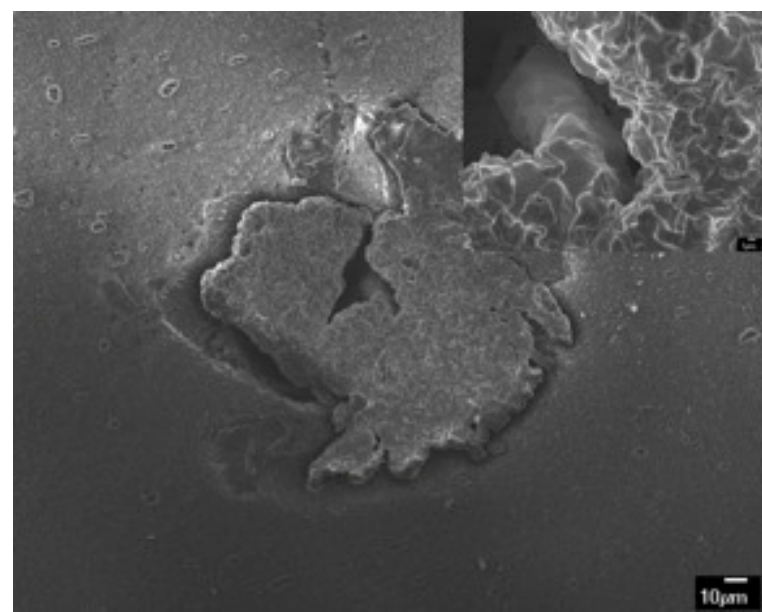

$2 \%$

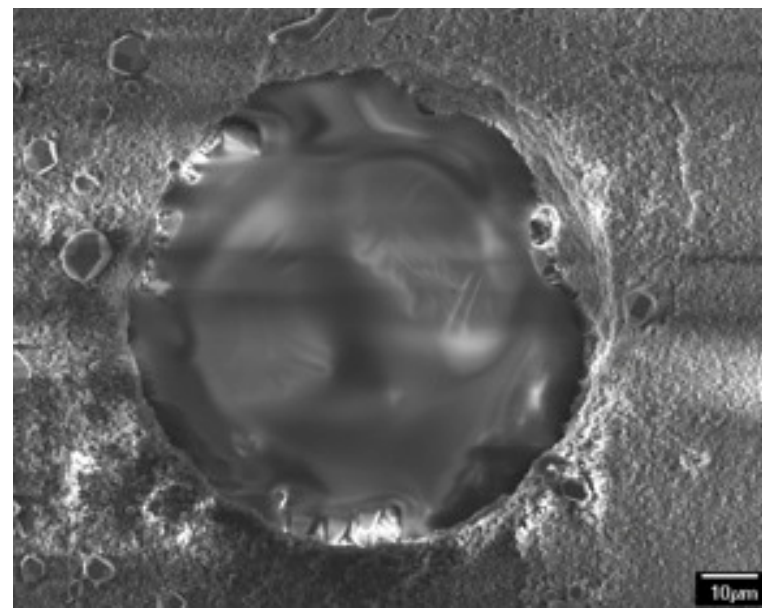

$10 \%$

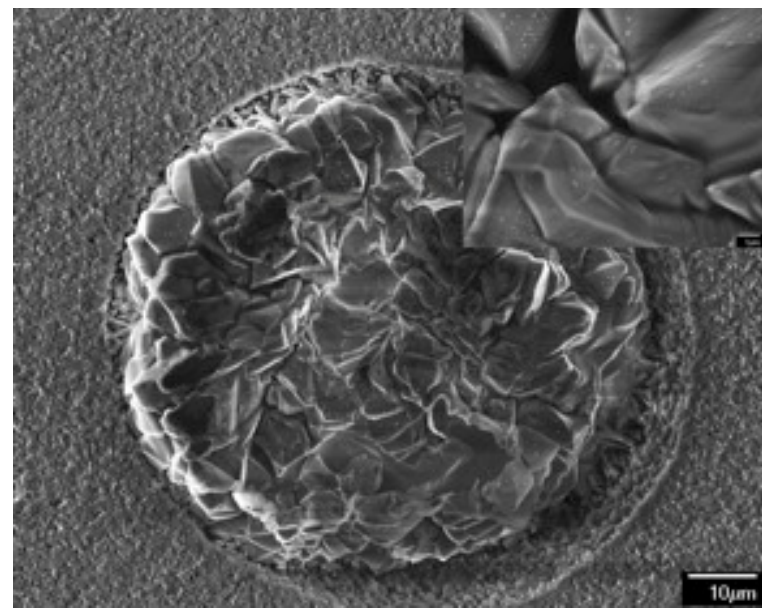

$50 \%$

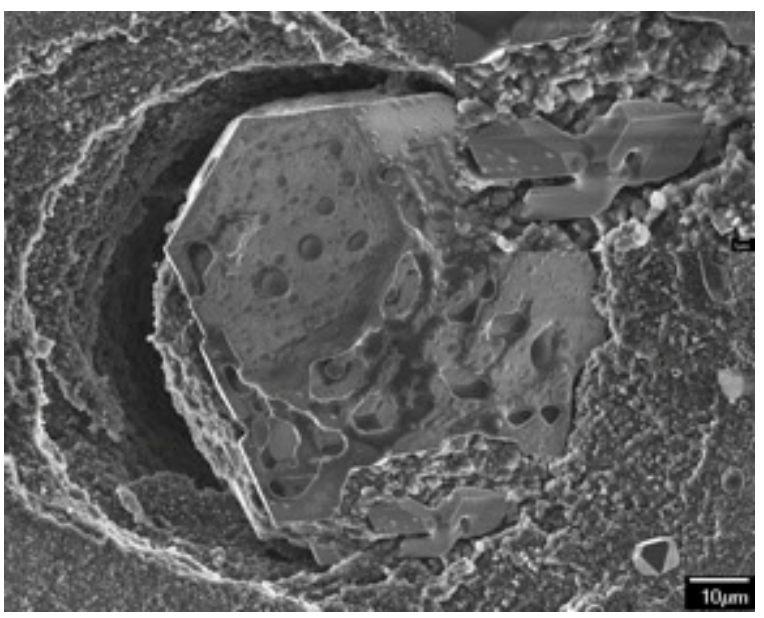

$5 \%$

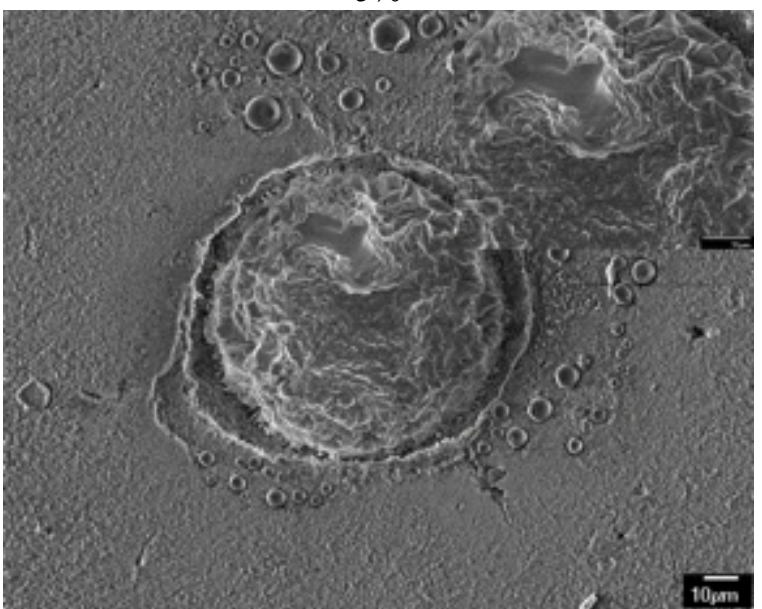

$25 \%$

Figure 7-11 SEM image of Pt-4 platinum via fired at different hydrigen concentration in the mixture with argon. Feedthroughs are fired at $1400{ }^{\circ} \mathrm{C}$. High magnification image shown in left corner

In the lowest hydrogen concentration, platinum only densified and no structure was

observed. High magnification images also shown in figure 7-11 indicate the sample fired in 2\% 
hydrogen showed that the platinum structure is very dense and all the particles almost fuse together. By increasing the concentration of hydrogen up to $5 \%$, all platinum particles fuse together and create one large platinum single crystal with the truncated octahedron shape. Figure 7-9 also shows a truncated octahedron crystal formed in the via fired in the $\mathrm{H}_{2}-\mathrm{Ar}$ (5-95) atmosphere. In addition to that, alumina starts to dissolve into the platinum. Because the low firing temperature, alumina grains maintained their structure and grain growth is not observed.

An alumina coating formed on the surface of the platinum after the hydrogen concentration reached $25 \%$. Increasing the hydrogen concentration from 25 to $50 \%$ in $1400{ }^{\circ} \mathrm{C}$ helps the alumina covers the surface of platinum completely. The sample fired in 50\% hydrogen has a more ordered structure than the other ones. Alumina has moved from the sidewalls to the via and to the surface. This behavior also recessed the alumina around the via and developed a mechanical bump to the top structure.

The microstructure of the sample in 50\% hydrogen is very similar to the structure of the sample fired at $1450{ }^{\circ} \mathrm{C}$ in $25 \%$ hydrogen in figure $7-5$. The temperature and the concentration are acting in the same way. Increasing both the temperature and hydrogen concentration increases the amount of dissolved alumina into the platinum. Shankland and Waff [150] for the first time showed that hydrogen decreases the melting temperature of the material in the earth crust, which is known as partial melting phenomena. Grönbeck et al. [147] showed that the introduction of hydrogen into palladium clusters could soften the Pd-Pd bond and would decrease its melting temperature. In addition, hydrogen also induces the melting of materials due to the heat released during the dissociative adsorption. In conclusion, it could be said that hydrogen increases the possibility of melting and dissolution of alumina into platinum. By increasing the hydrogen concentration, the amount of alumina increases, but due to limited solid 
solubility, the platinum cannot hold that much alumina and it floats to the surface. The sample fired in $10 \%$ hydrogen is compatible with this mechanism. As depicted in figure $7-9$, the $10 \%$ sample shows that only platinum is melted, but there is no sign of ceramic around or above the platinum via. The hydrogen concentration is sufficient to absorb alumina into platinum, minimizing the "slag" effect to the surface. This result is also in agreement with the sample shown in figure $7-5$ fired at $1550{ }^{\circ} \mathrm{C}$. The sample fired at $1400{ }^{\circ} \mathrm{C}$ and $10 \%$ hydrogen is very similar to the sample fired at $1550{ }^{\circ} \mathrm{C}$ and $25 \%$ hydrogen. At $1550{ }^{\circ} \mathrm{C}$, the sample has a better chance of melting and creating a melted dense structure. The size of the via at $1400{ }^{\circ} \mathrm{C}$ is $85 \mu \mathrm{m}$, while at $1550{ }^{\circ} \mathrm{C}$, it is $104 \mu \mathrm{m}$. This means that at $1550{ }^{\circ} \mathrm{C}$ and $25 \%$ hydrogen concentration, the amount of absorbed alumina is more than that of the sample fired at $1400{ }^{\circ} \mathrm{C}$ and $10 \%$ hydrogen.

In the filling process, the area surrounding the via is also covered with ink. In the burn-out process and during the heating, the platinum covering this surrounding area starts to coalescence and fuse together. The particles start out as drops with no distinct structure. Upon heating, the particles change to a macro-sized single crystal with a distinct crystal shape. Particles formed in different hydrogen concentrations are depicted in figure 7-11. Lee et al. [151] showed that below the melting temperature, platinum basically has a spherical shape with distinct (100) and (111) facets, and curved regions in between. They also observed the formation of cubo-octahedrons shape particles when samples were heavily contaminated with carbon. Particles heated at $1400{ }^{\circ} \mathrm{C}$, about $350^{\circ} \mathrm{C}$ degrees lower than the melting temperature of the platinum, demonstrate particle that are faceted. It is clear that increasing the hydrogen concentration changes the particle shape from the truncated octahedron to the spherical faceted in some edges to the almost spherical particle. Truncated octahedron is the equilibrium FCC crystal shape at $0^{\circ} \mathrm{K}[152,153]$, which means that increasing the hydrogen concentration enhances the atom movements and 
helps the platinum crystal to transfer from $0^{\circ} \mathrm{K}$ equilibrium state to the spherical shape. The faceted surfaces observed in the sample fired in $10 \%$ hydrogen are not really flat and have a ledge structure. These stepped and kinked structures suggest that the surface moves to form a complete sphere [151]. At 25\% hydrogen concentration, almost all of those ledges disappear and crystal has a complete spherical shape.

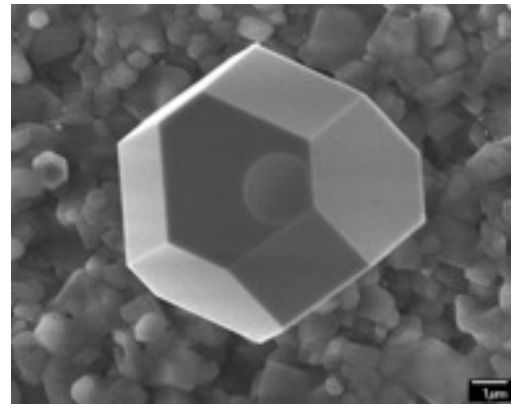

$5 \%$

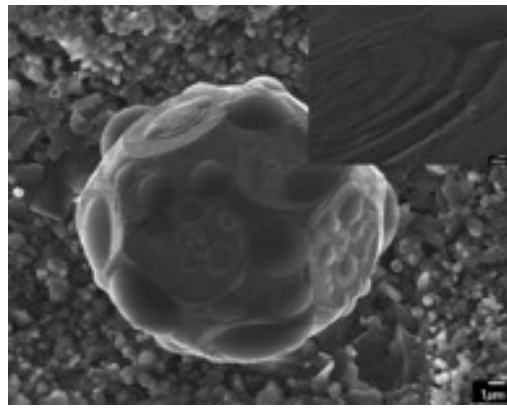

$10 \%$

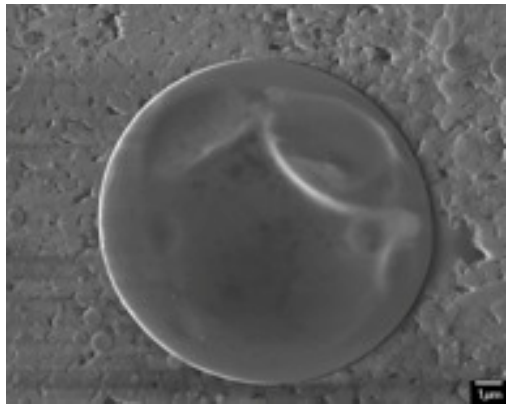

$25 \%$

Figure 7-12 High magnification SEM image of platinum crystals formed in different hydrogen concentration at $1400{ }^{\circ} \mathrm{C}$

\subsection{Atmosphere humidity}

Ratio of the $\mathrm{H}_{2} \mathrm{O}$ to $\mathrm{H}_{2}$ impacts the reaction between platinum and alumina due to the partial pressure of $\mathrm{O}_{2}$. $\mathrm{H}_{2} \mathrm{O}$ also controls the oxidation of platinum and reduction of alumina [10]. Figures 7-13 and 7-14 are shown the effect of wet hydrogen atmosphere on the structure of Pt-4 and Pt-5 platinum particles. Hydrogen passes through the bubbler to burn-out the organic. After the temperature reached $600^{\circ} \mathrm{C}$ the concentration of the wet hydrogen is decreased to decrease the amount of $\mathrm{H}_{2} \mathrm{O}$ vapor in the furnace and reduces the oxygen partial pressure to zero. Samples were heated up to $1550^{\circ} \mathrm{C}$ at the rate of $10^{\circ} \mathrm{C} / \mathrm{min}$ and were kept for 3 hours and then were cooled down with the same rate. 
The $\mathrm{H}_{2} \mathrm{O}$ reacts with platinum, minimizing the platinum densification. Additionally, $\mathrm{H}_{2} \mathrm{O}$ minimizes alumina dissolution into platinum. The final structure of via is similar to a sintered powder.

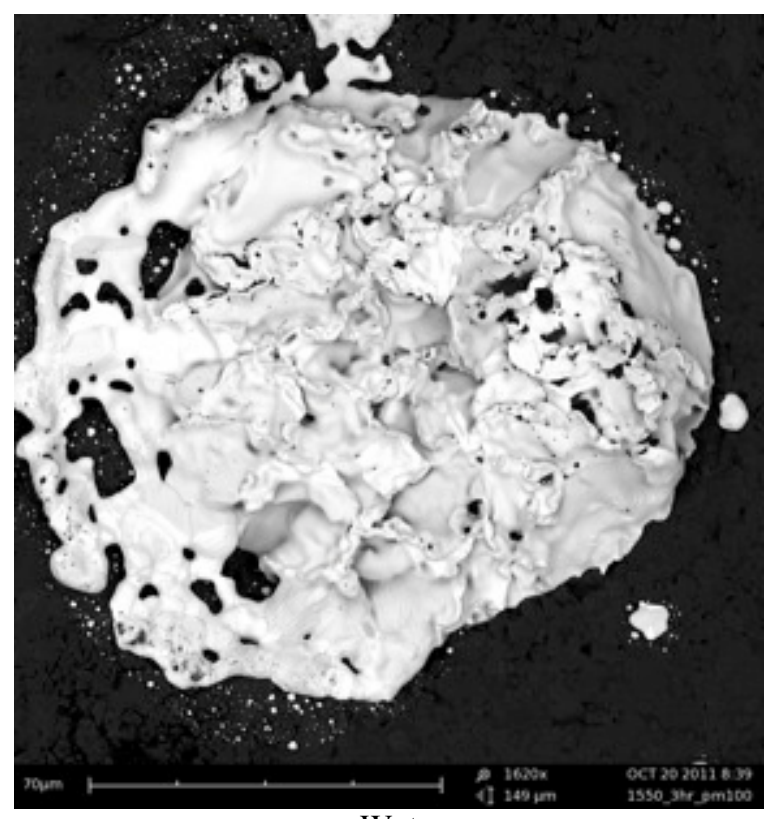

Wet

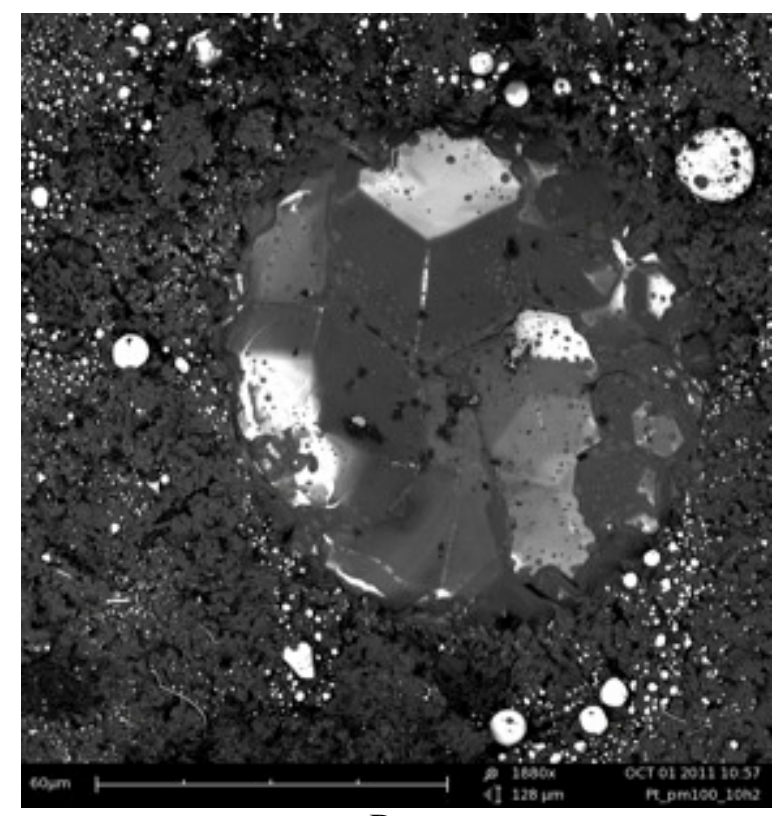

Dry

Figure 7-13 Back scatter SEM image of Pt-4 feedthough sample fired at $1550{ }^{\circ} \mathrm{C}$ and $10 \% \mathrm{Hr}-90 \%$ Ar in wet and dry atmosphere

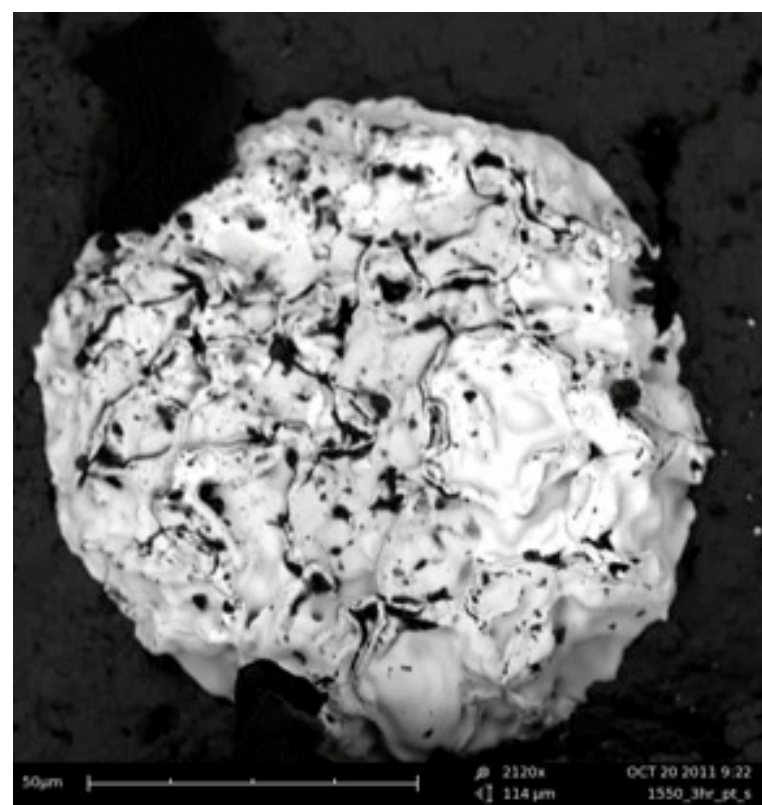

Wet

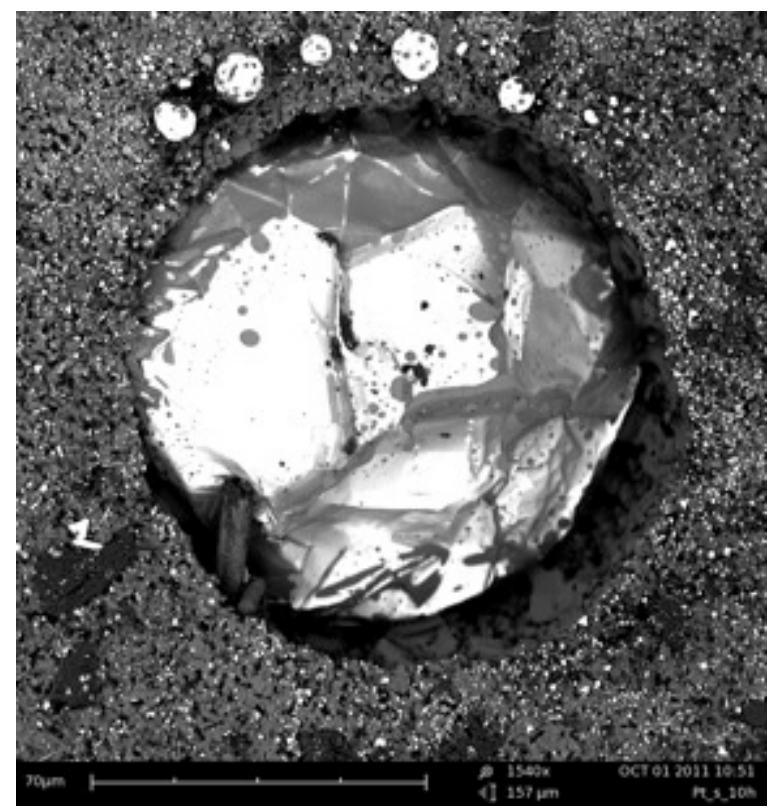

Dry

Figure 7-14 Back scatter SEM image of Pt-5 feedthrough samples fired at $1550^{\circ} \mathrm{C}$ and $10 \% \mathrm{Hr}-90 \%$ Ar in wet and dry atmosphere 


\subsection{Platinum particle size}

One of the important parameter in sintering of platinum is the platinum particle size. Four different platinum particles were used to study the effect of particle size on the final structure of the feedthrough. Samples were fired in the $\mathrm{H}_{2}-\mathrm{Ar}(10-90)$ atmosphere at $1550{ }^{\circ} \mathrm{C}$. The SEM images of the fired feedthroughs are shown in figure 7-15.
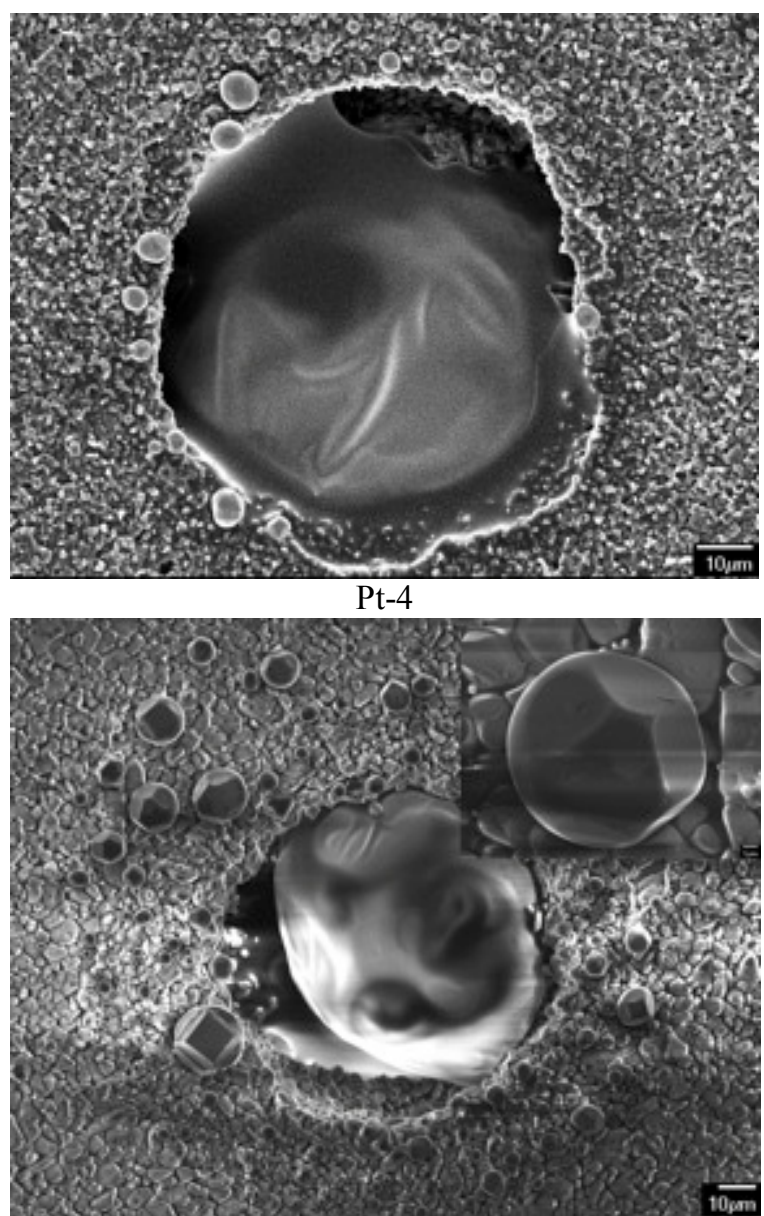

Pt-6

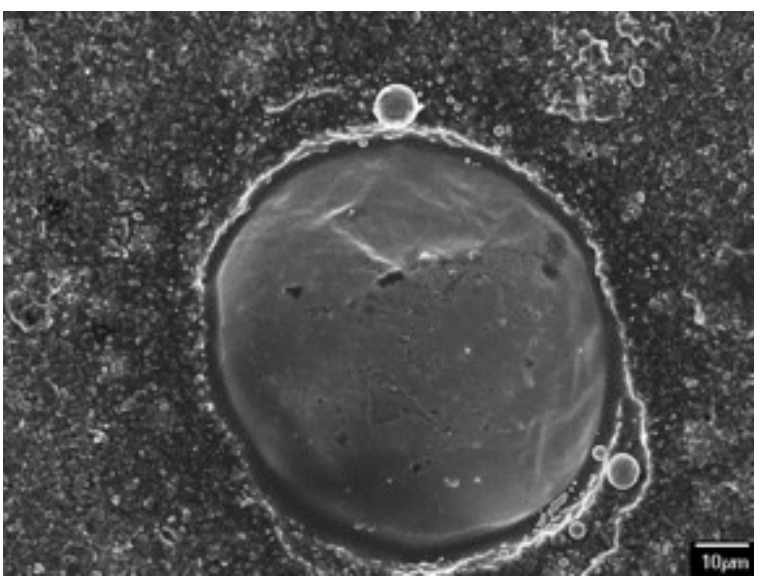

Pt-5

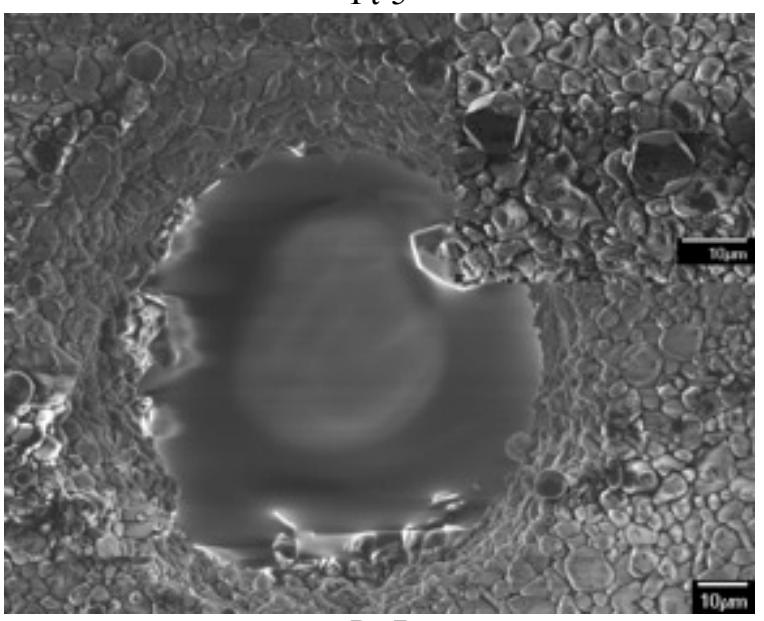

Pt-7

Figure 7-15 SEM image of platinum via with different platinum partcile size. Feedthroughs are fired in the $\mathrm{H}_{2}$-Ar (10-90) atmosphere at $1550{ }^{\circ} \mathrm{C}$

As can be seen from figure 7-15, Pt-5 and Pt-7 almost showed the same kind of microstructure. Both of them showed good bonding between platinum and alumina. This is not unexpected due to their similar densification behavior, physical structure and particle size. 
However, as shown in figure 7-16, the Pt-7 platinum powder contracts and lost the continuity through the length of the via.

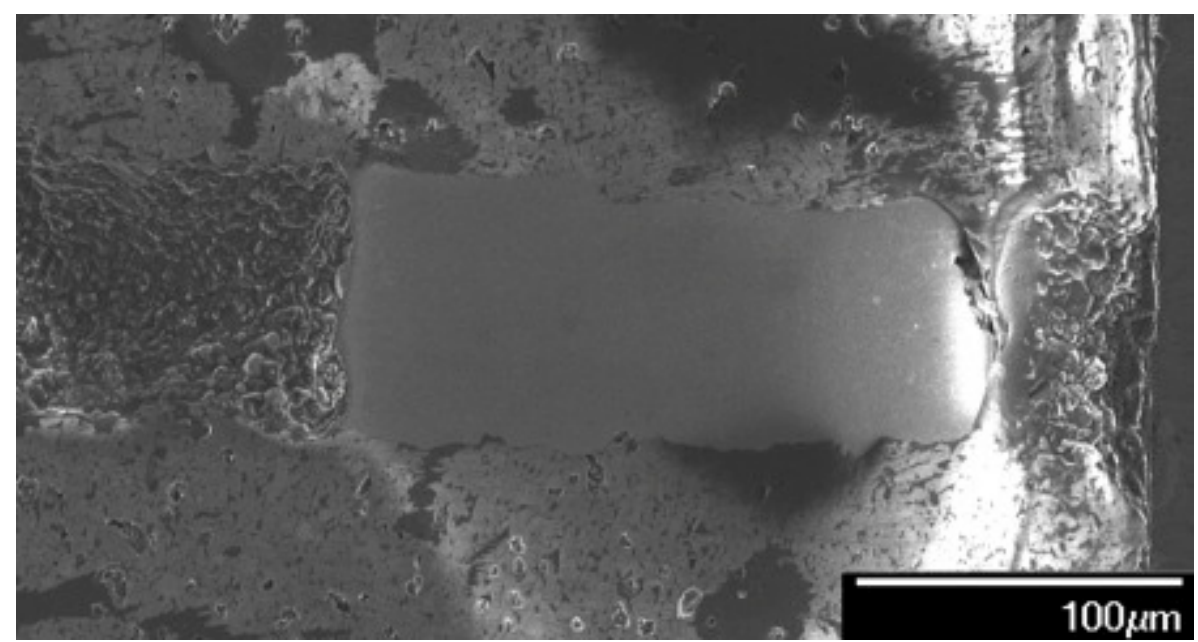

Figure 7-16 SEM image of the Pt-7 platinum powder. Feedthrough fired in the $\mathrm{H}_{2}-\mathrm{Ar}(10-90)$ atmosphere at $1550{ }^{\circ} \mathrm{C}$

The crystals formed around Pt-4, Pt-5 and Pt-6 have spherical shape with distinct (100) and (111) facets, while a truncated octahedron shape is observed for the Pt-7 particle. It is not clear why the truncated octahedron crystal was formed instead of the spherical crystal for Pt-7 particle. Crystals formed from Pt-4 platinum is more spherical in shape than the others. Higher surface energy of smaller particle helps the atoms move faster so the spherical particle forms in lower temperature.

Figure 7-17 shows the effect of introduction of 20\% Pt-4 in the microstructure of Pt-6 and Pt-7 feedthrough. Despite the fact that each platinum particle used in this project has it is own final microstructure; the mixture of Pt-6 and Pt-7 with Pt- 4 almost has the same structure. Upon heating the surface of the platinum via covered with the ceramic, Alumina is absorbed from the sidewalls and floats to the surface of the platinum. The grain structure of the alumina is very similar to the ceramic grain from the tape. This could support the migration mechanism of alumina particles from the side of the via to its surface. 


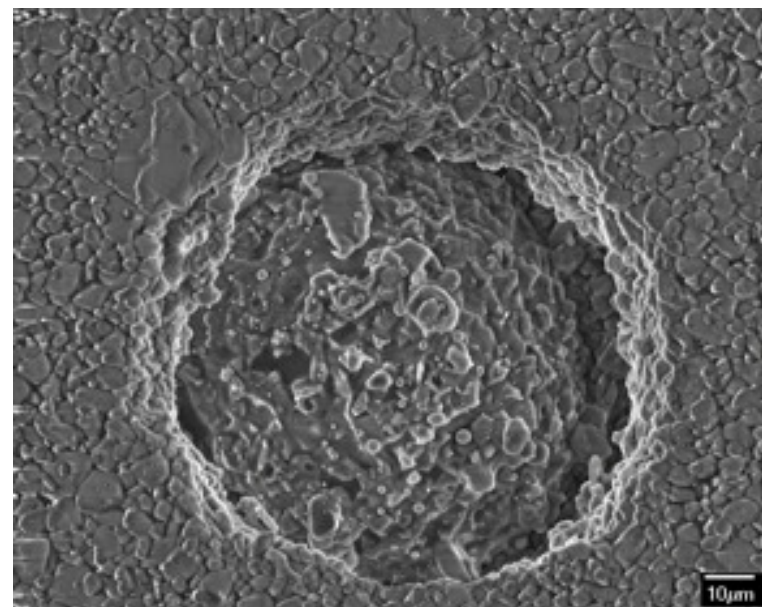

Pt-6 + 20\% Pt-4

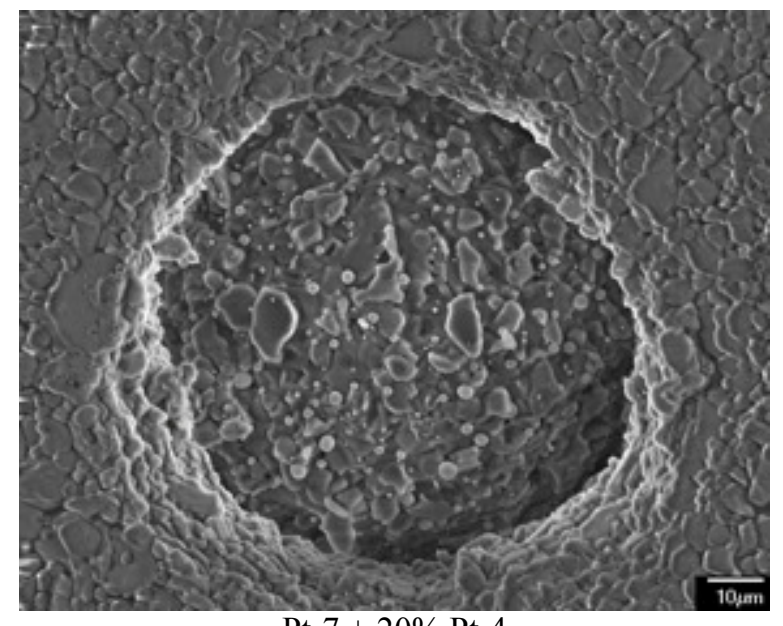

Pt-7 + 20\% Pt-4

Figure 7-17 SEM image of platinum via with the mixture of different platinum particles. Feedthroughs are fired in the $\mathrm{H}_{2}$-Ar (10-90) atmosphere at $1550{ }^{\circ} \mathrm{C}$

The cross section image of Pt-7 and Pt-7 + 20\% Pt-4 samples are shown in figure 7-18.

The Pt-7 platinum powder melted on the top surface and wets the sidewalls of the alumina

completely. However, in mixture of Pt-7 and Pt-4 sample a layer of ceramic covered the surface of the platinum powder as showed before in figure 7-17. The EDS analyses indicated that the coating composed mostly out of alumina. The diffusion gradient of the elements form the platinum/ alumina interface is shown in figure 7-19.

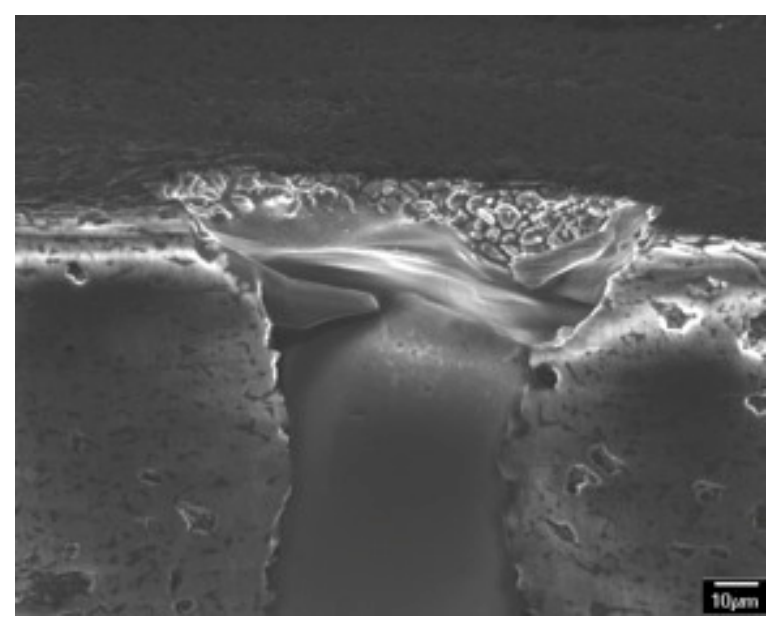

Pt-7

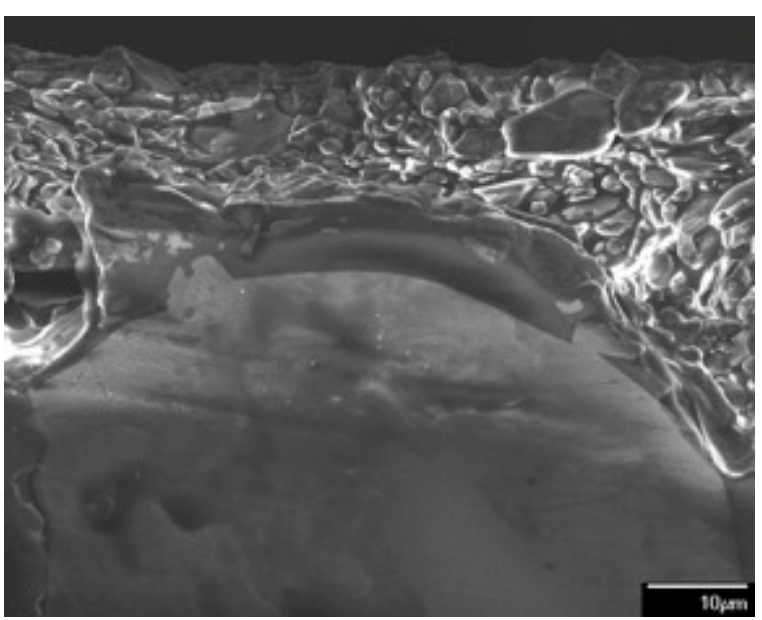

Pt-7 + 20\% Pt-4

Figure 7-18 SEM image of Pt-7 platinum via cross-section. Feedthroughs are fired in the $\mathrm{H}_{2}$-Ar (10-90) atmosphere at $1550{ }^{\circ} \mathrm{C}$ 
Aluminum, silicon and oxygen exist in the first 15 microns of the platinum via. This suggests that in this region platinum is mixed with silica and alumina. The concentrations of aluminum and oxygen decrease until they become negligible after 15 microns. Concentration of silicon increased and reached to its maximum in 25 microns depth from the interface. As showed before in in figure 7-8 and 7-9, silicon prevents the diffusion of aluminum into platinum, consistent with the aluminum concentration decreasing with increasing silicon showed in figure 7-18. As the distance increases from the interface, the concentration of the silicon decreases.

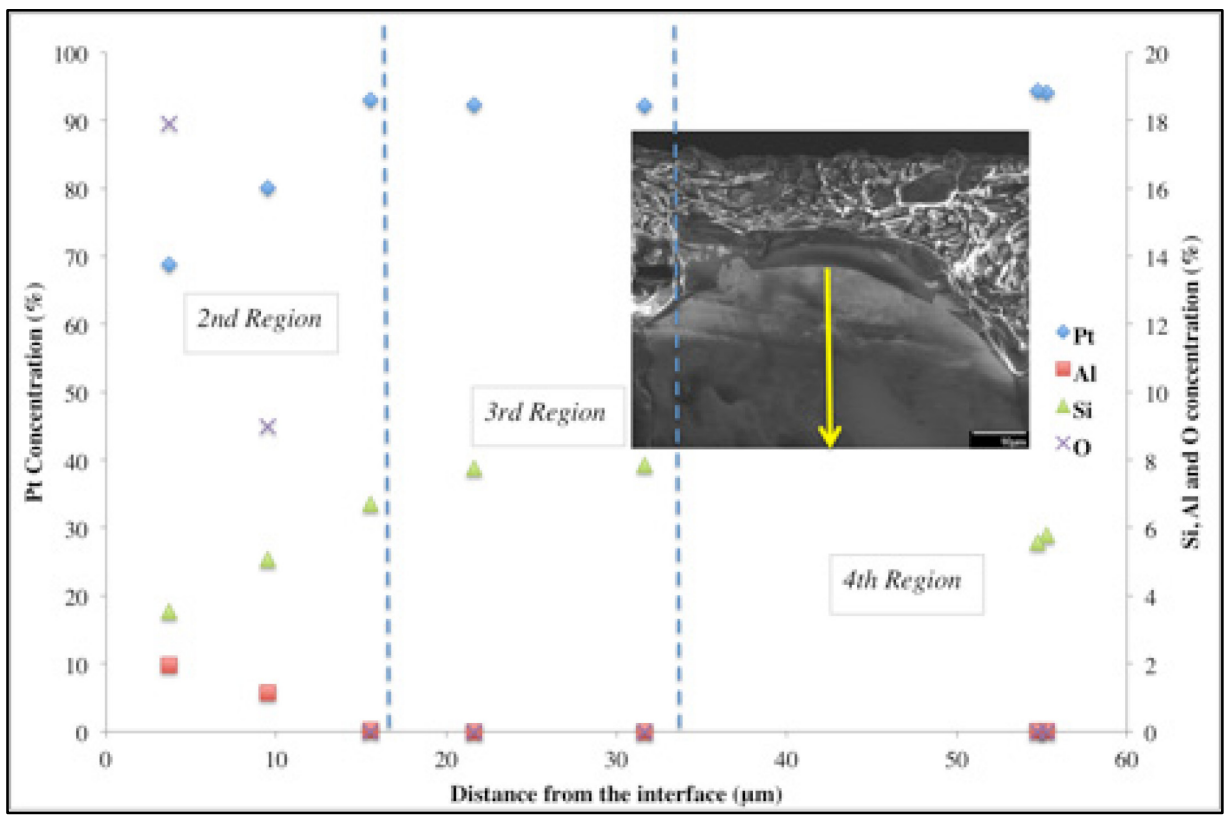

Figure 7-19 Concentration gradient of different element in platinum for the Pt-7 + 20\% Pt-4 platinum powder. Feedthrough fired in the $\mathrm{H}_{2}-\mathrm{Ar}(25-75)$ atmosphere at $1550{ }^{\circ} \mathrm{C}$

As explained previously, platinum dissolves the ceramic from the sidewall, reacts with a portion of it and push the rest of it to the top. Due to this process, the via could be spilt into four regions. In the first region, a ceramic coating forms on the top surface of the via. After that, in the first few microns from the interface a mixture of platinum and ceramic develops in the second region. In the third region, 20 -30 micron from the top surface, aluminum and oxygen and silicon reached to its maximum concentration. Afterward silicon concentration decreased 
and maintained steady with its equilibrium concentration in the fourth region, as shown in figure 7-19.

\subsection{Platinum metallization}

To control the shrinkage of the platinum and increase the platinum/alumina bonding strength, different metallization have been used in the ink formulation. Alumina, yttrium oxide, iridium and rhodium were used before in ink formulation in the natural atmosphere. However, none of them was successful.

\subsubsection{Oxide metallization}

Ceramic oxide could be used in the ink formulation to control the shrinkage of platinum. As discussed before in section 6.7.2 alumina is the first choice, because of the similarity with ceramic tape. In a reduced atmosphere, tungsten oxide could react with platinum and prevent its densification. A series of feedthroughs were prepared from the platinum with $20 \%$ vol. oxide in ink formulation and were fired in $1550^{\circ} \mathrm{C}$. The results are shown in figure 7-20.

The surface of the platinum in the sample with $20 \%$ alumina is completely covered with the alumina. The ink completely filled the via, yet the alumina migrated and covered the surface. In the sample with $20 \%$ tungsten oxide, platinum in the via pulled back from the wall. It seems that nano-sized oxide particles filled the platinum's porosity and completely mix with platinum at high temperature. Yet, tungsten oxide did not control the shrinkage of the platinum. In pure platinum, as shown in figure 7-3, platinum interacts with alumina and absorbs it into the via. Platinum seems to react with tungsten oxide instead of alumina at high temperature and the small grain size of tungsten oxide does not control the shrinkage so the final structure is smaller than the via size. 


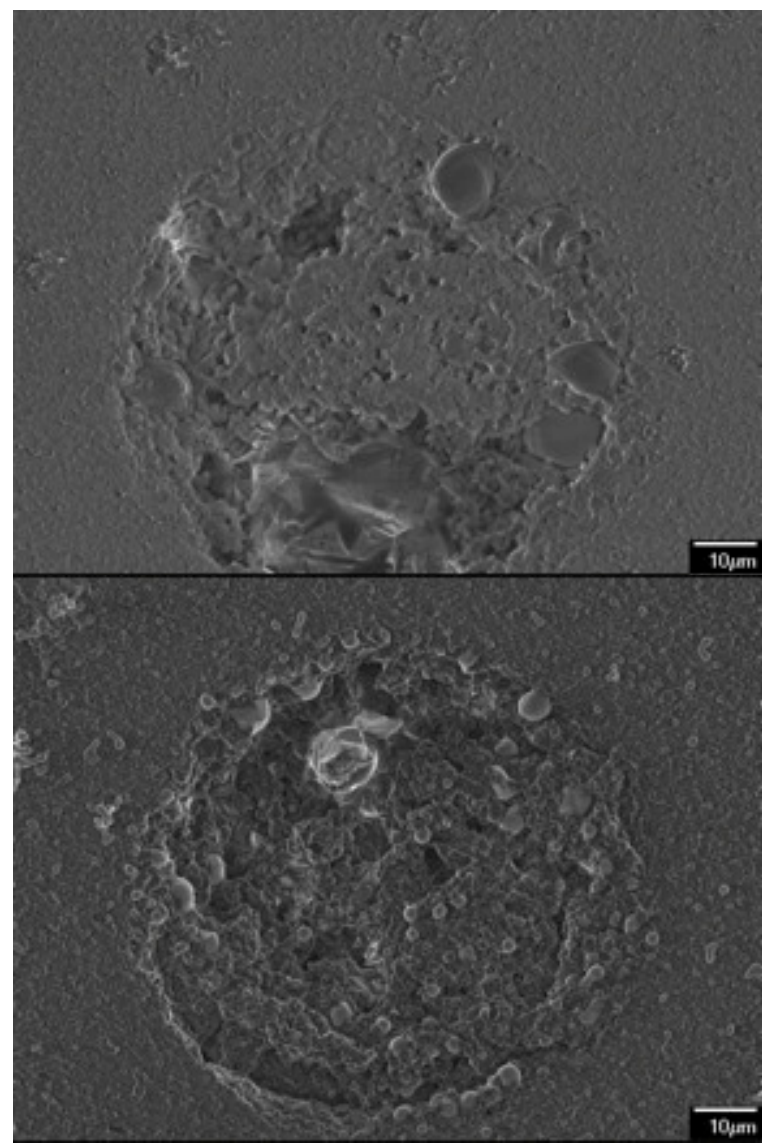

$\mathrm{Al}_{2} \mathrm{O}_{3}$
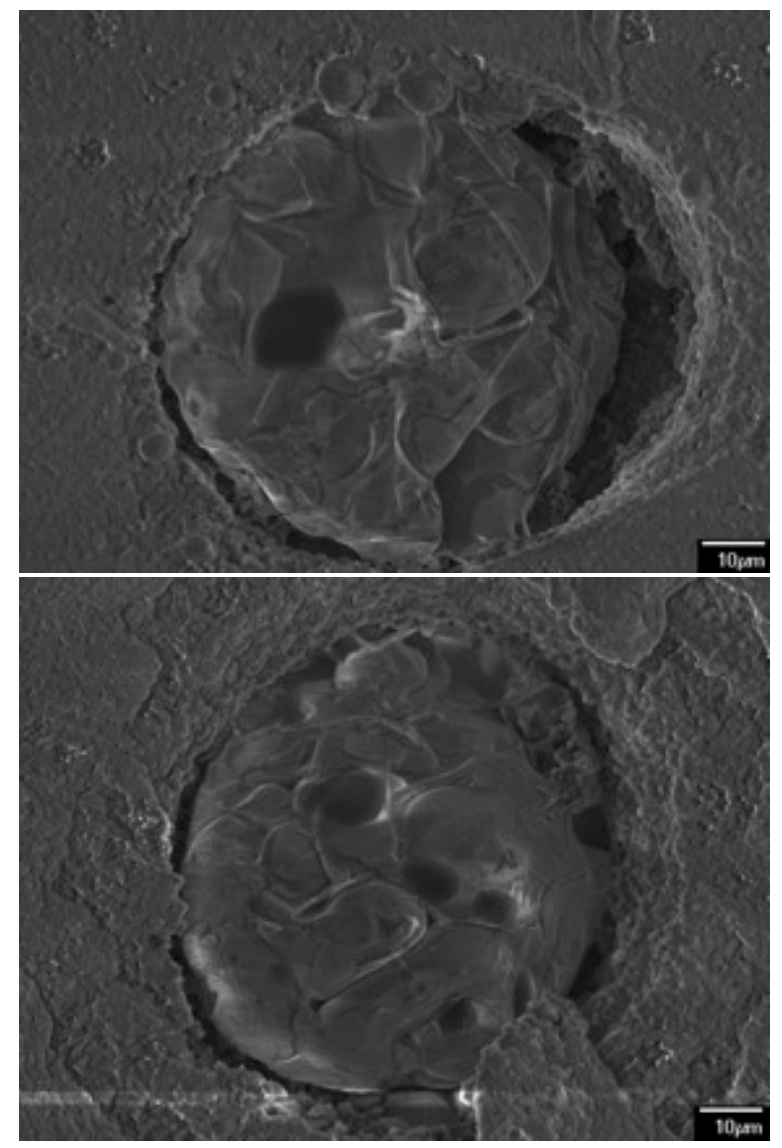

$\mathrm{WO}_{3}$

Figure 7-20 SEM image of platinum metalized with aluminum oxide and tungsten oxide. Samples fired in the $\mathrm{Hr}$-Ar (10-90) atmosphere at $1550{ }^{\circ} \mathrm{C}$

\subsubsection{Tungsten metallization}

Refractory metal elements, such as tungsten and molybdenum, have a long history in cofired multi layer ceramic industry [67]. Densification behavior and thermal expansion of refractory metals and alumina ceramic is very similar and they have been used to fabricate the internal metallization of cofire alumina microcircuits. However, in the case of the refractory elements, the very high melting points as well as their resistance to deformation impact the sintering at lower temperatures below their melting points. Therefore platinum has been used as a sintering aid for lowering the sintering temperature of refractory metals [154]. This history makes tungsten/platinum an ideal choice for ink metallization for firing in a reduced atmosphere. 
Microstructures of mixture of W-1 with Pt-4 particles with different ratio are shown in figure 7-

21. In addition to that, densification of each mixture is shown in figure 7-22.

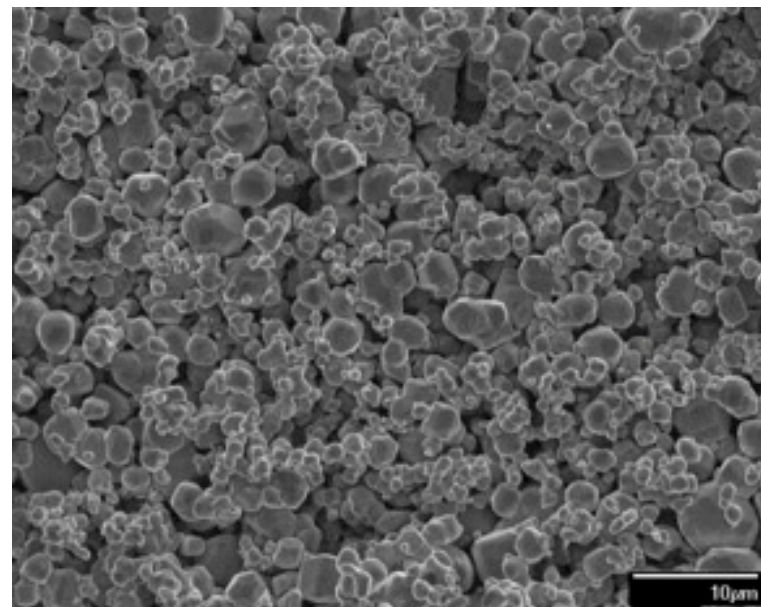

$\mathrm{W}-1$

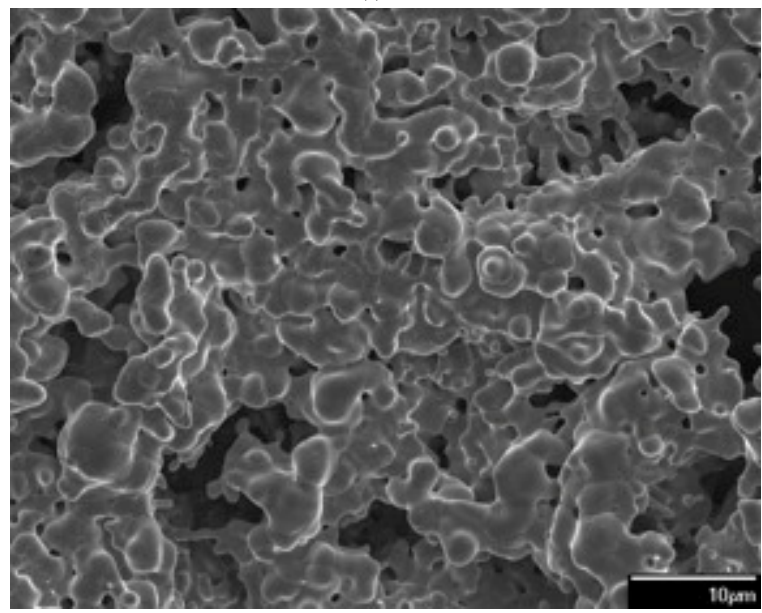

$\mathrm{W}-1+50 \% \mathrm{Pt}-4$

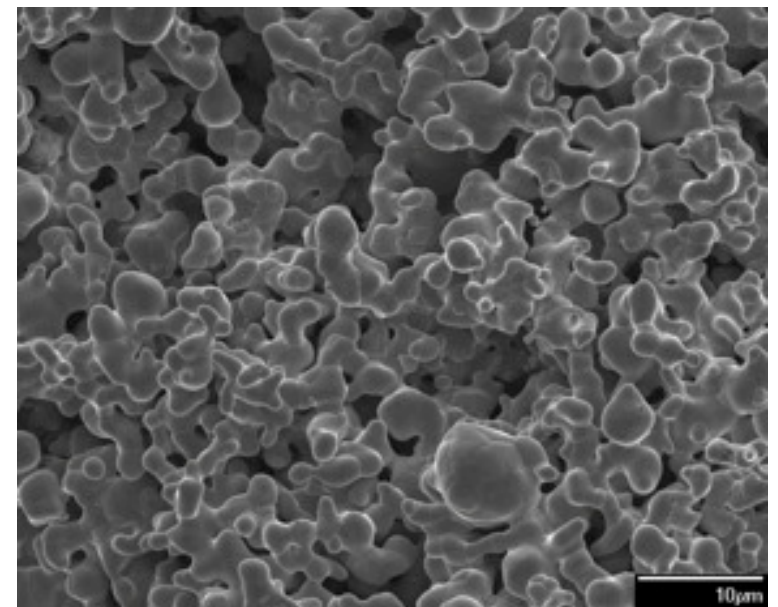

$\mathrm{W}-1+20 \% \mathrm{Pt}-4$

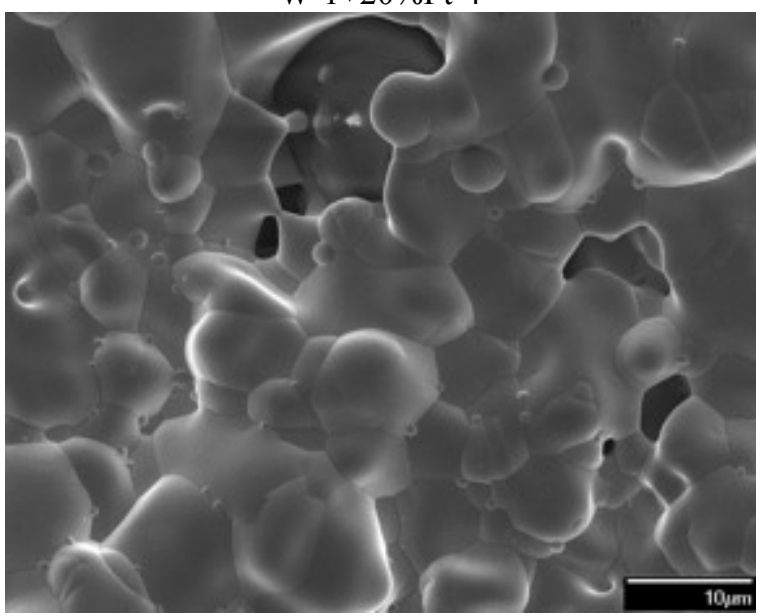

$\mathrm{W}-1+80 \% \mathrm{Pt}-4$

Figure 7-21 Microstrcture of the mixture of W-1 and Pt-4 partciles in different ratio. Samples fired in the $\mathrm{H}_{2}$ - Ar (10-90) atmosphere at $1550{ }^{\circ} \mathrm{C}$

Increasing the amount of platinum in mixture increases the densification of the tungsten.

The metal grains are larger in the mixture 80-20 (Pt-W) mixture and the other two mixtures. This behavior is also in agreement with densification behavior that is shown in figure 7-22. Pure platinum contracts up to $65 \%$ of its original density in hydrogen, while adding $20 \%$ tungsten decreases the densification up to $37 \%$. The tungsten powder shrinks about $5 \%$. Addition of $20 \%$ and $50 \%$ platinum to tungsten increase its shrinkage up to 15 and $22 \%$. The tungsten powder 
with $20 \%$ platinum is the optimum concentration to start with. The resultant feedthrough made from the tungsten with $20 \%$ wt. platinum is shown in figure $7-23$.

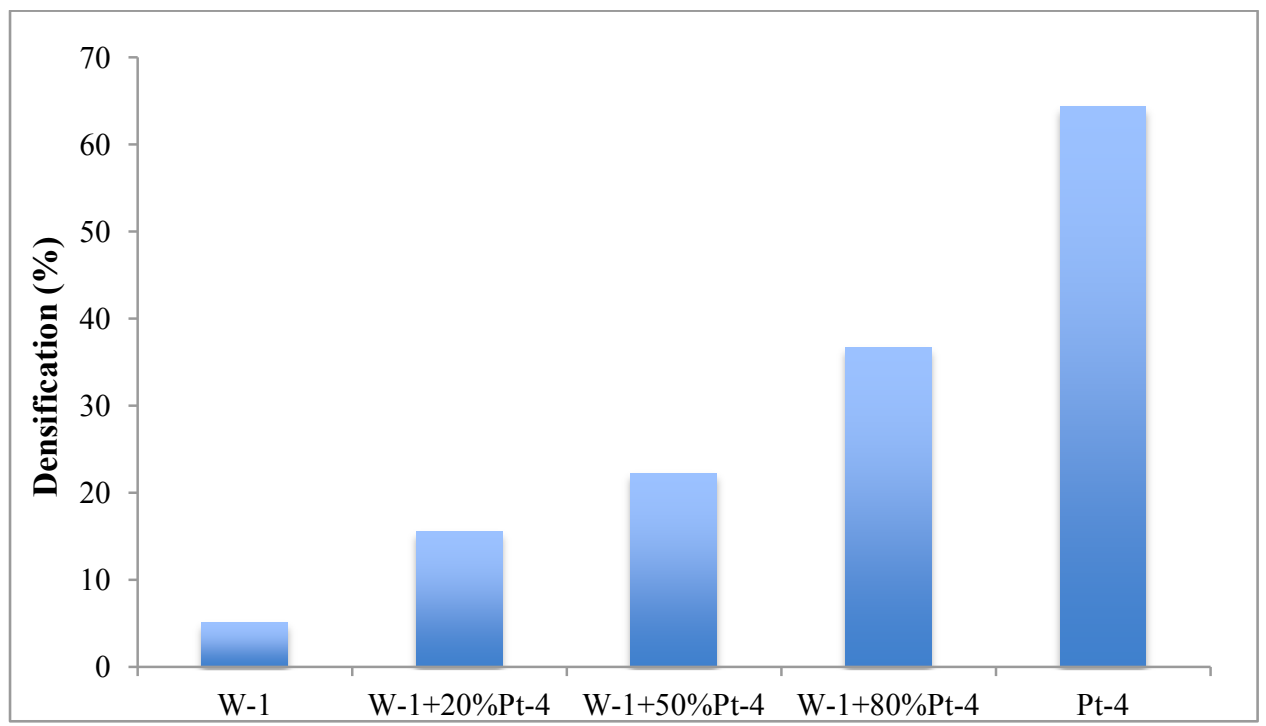

Figure 7-22 Densification of the mixture of W-1 and Pt-4 partciles in different ratio . Samples fired in the $\mathrm{H}_{2}-$ $\operatorname{Ar}(10-90)$ atmosphere at $1550{ }^{\circ} \mathrm{C}$

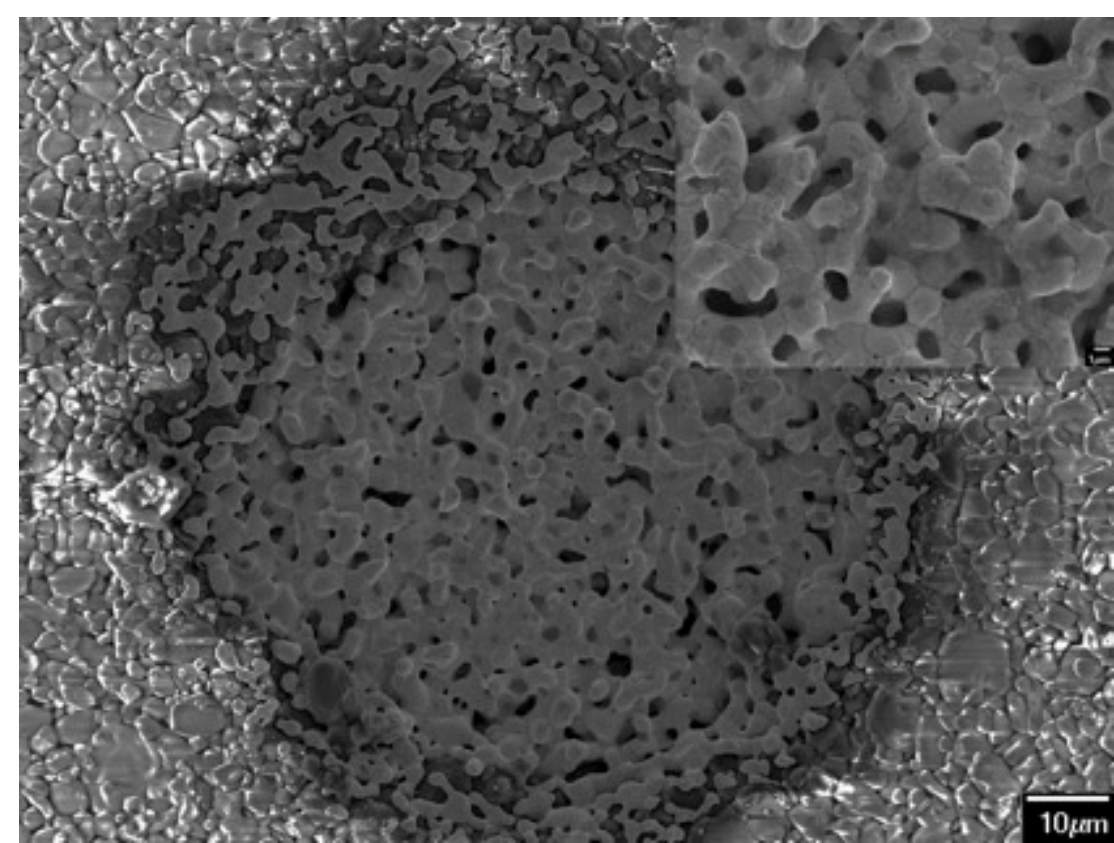

Figure 7-23 SEM image of tungsten metalized with platinum (W-1 + 20\% Pt-4). Feedthrough fired in the $\mathrm{H}_{2}-$ Ar (10-90) atmosphere at $1550{ }^{\circ} \mathrm{C}$. High magnification image shown in left corner

The metal particles filled the via completely. Tungsten particles partial sinter completely and the structure has sufficient density to produce hermeticity. To increase the density of the via, 
instead of large micron-sized particles $(\sim 1-5 \mu \mathrm{m})$, submicron tungsten particle $(>1 \mu \mathrm{m})$ was used. The microstructure of the fired sub micron tungsten with platinum (W-2 $+20 \%$ Pt-4) is shown in figure 7-24.

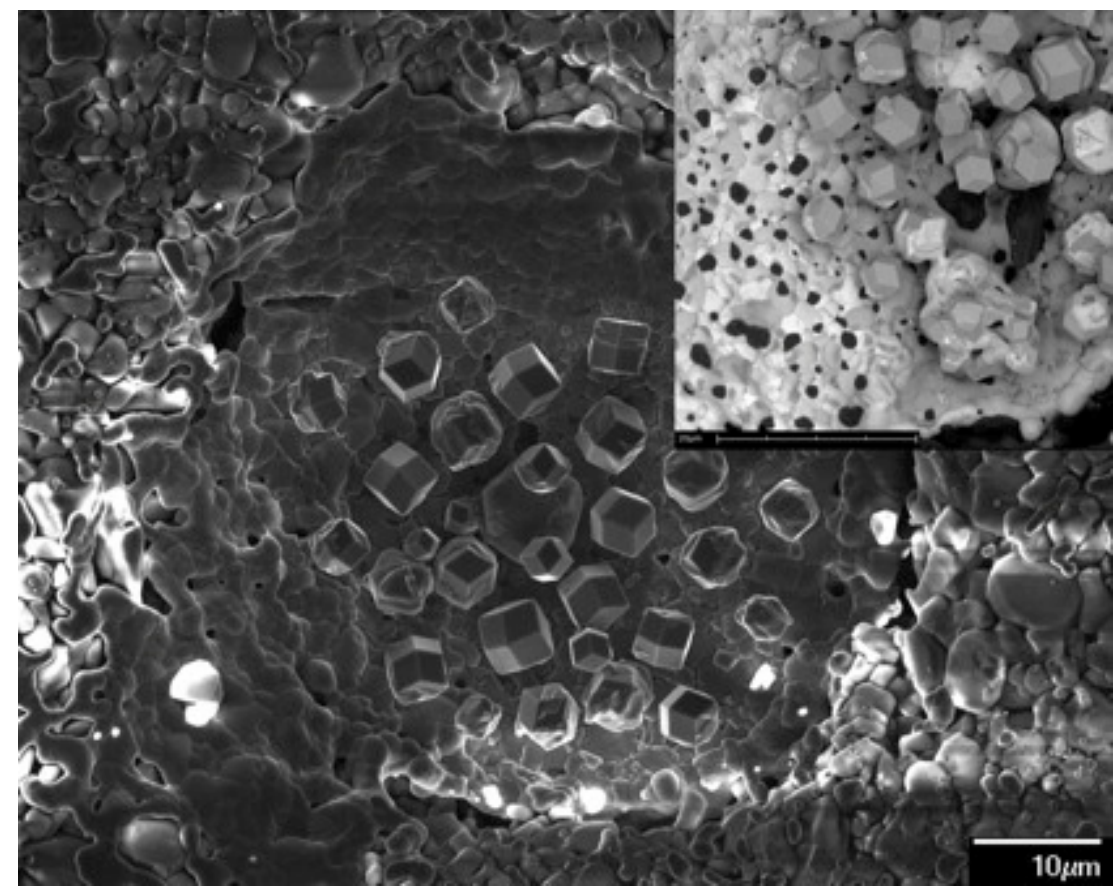

Figure 7-24 SEM image of submicron tungsten metalized with platinum (W-2 + 20\% Pt-4). High magnification image in left corner shows the backscatter image. Feedthrough was fired in the $\mathrm{H}_{2}-\mathbf{A r}(10-90)$ atmosphere at $1550{ }^{\circ} \mathrm{C}$

Decreasing the tungsten particle increases the densification of the tungsten/platinum mixture. In addition to different densification behavior, some crystal was formed on top of the via in small tungsten particle. Backscatter image of the W-2/Pt-4 sample in figure 7-24, showed that the crystals formed are not pure tungsten. Okuyama [155] showed that pure tungsten crystal has the rhombic dodecahedron shape, which is similar to some of the particles formed in W-2/Pt4 sample. Darker particles in figure 7-24 depicted a rhombic dodecahedron crystals. However, when platinum reacts with the tungsten, the crystals start to deform from rhombic dodecahedron to the spherical shape. Additionally, platinum acts as the filler material and attaches some of the tungsten crystal to each other. 
Platinum and tungsten are both biocompatible, however, the difference in atomic electron structure could create corrosion cell in the body and increase the possibility of the feedthrough failure. Hence, despite the fact that W-2 with 20\% Pt-4 has the dense structure, the via material with a greater concentration of the platinum is desirable. The results of $\mathrm{W}-2 / \mathrm{Pt}-4$ sample with different ratios are shown in figure 7-25. Even though the via structure looks completely dense in both cases, the platinum shrank, detached from the side of the via and providing lack of hermeticity.

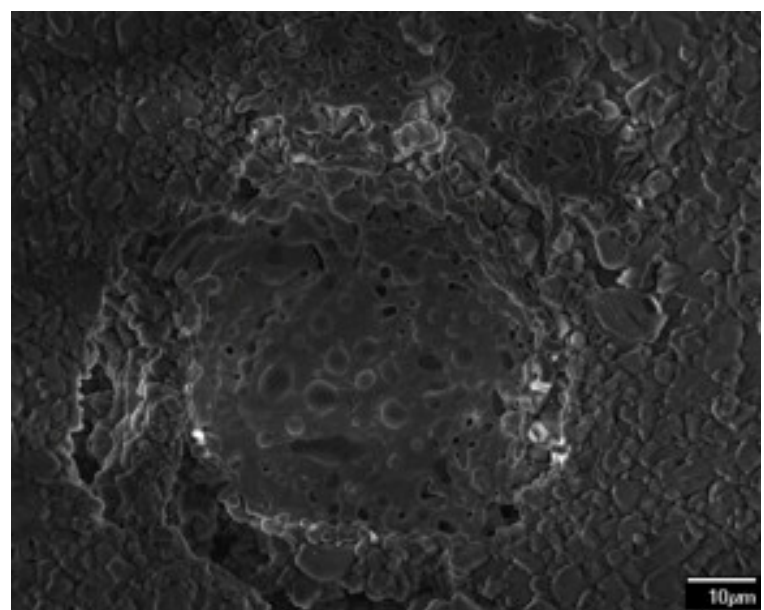

$\mathrm{W}-2+50 \%$ Pt- 4

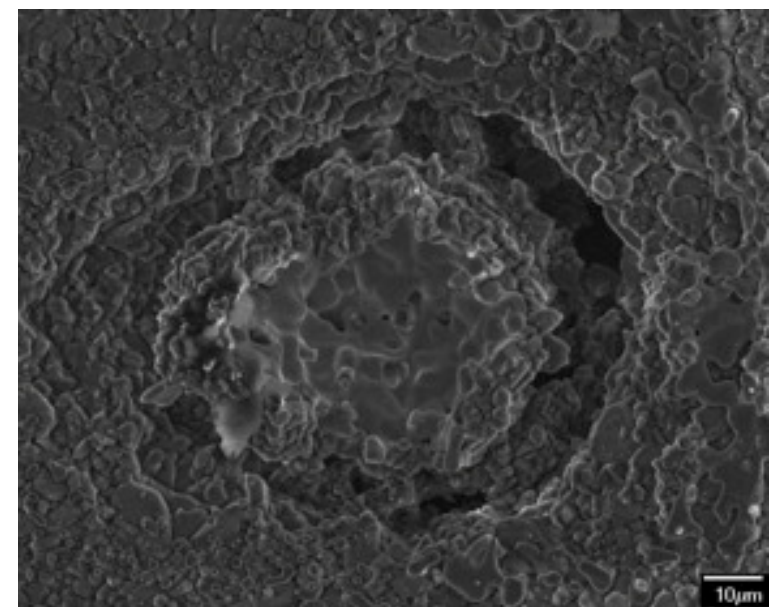

$\mathrm{W}-2+80 \%$ Pt-4

Figure 7-25 SEM image of submicron tungsten metalized with platinum (W-2 / Pt-4). Feedthrough was fired in the $\mathrm{H}_{2}-\mathrm{Ar}(10-90)$ atmosphere at $1550{ }^{\circ} \mathrm{C}$

To enhance the structure of the via, the mixture of $\mathrm{W}-2 / \mathrm{Pt}-5$ with two different composition (i.e. 50-50 and 20-80) were prepared and fired under the same condition. The images of fired vias are shown in figure 7-26. In addition the cross section image of 50-50 sample is also shown in figure 7-27. 


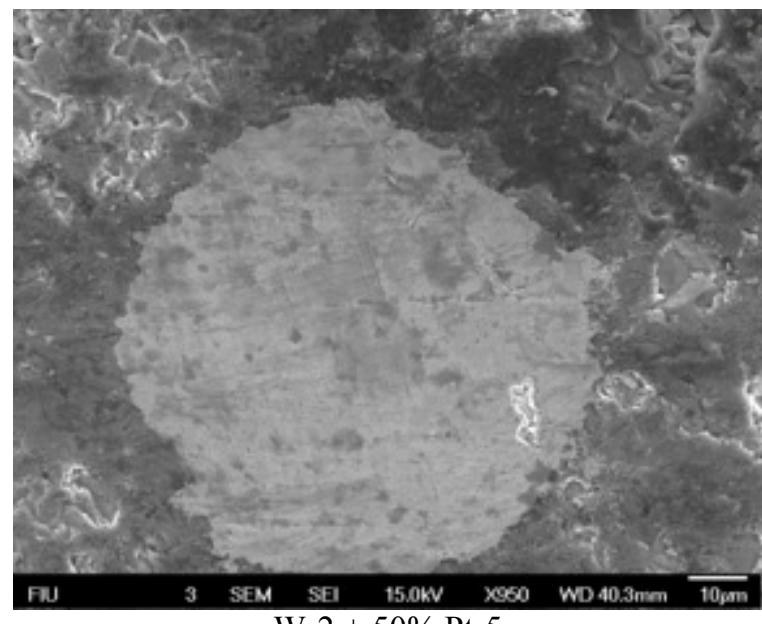

$\mathrm{W}-2+50 \%$ Pt-5

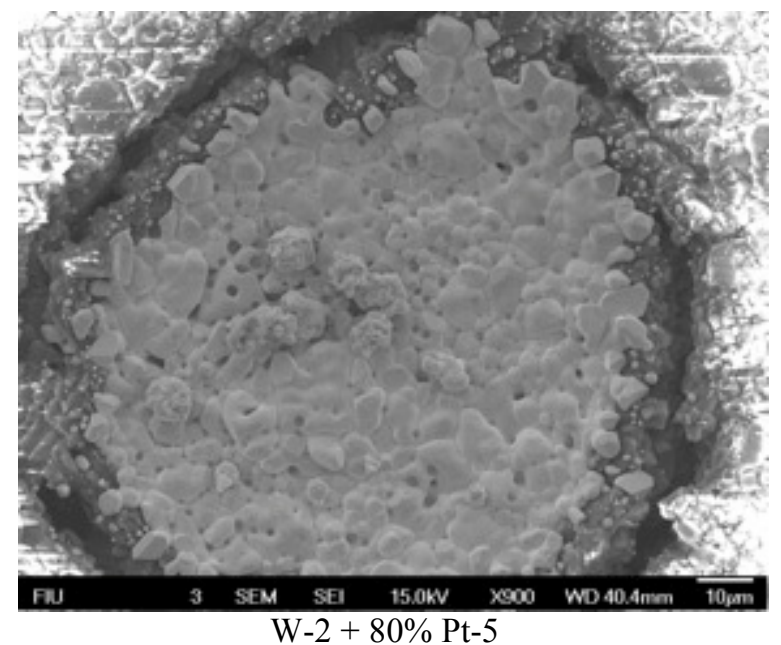

Figure 7-26 SEM image of submicron tungsten metalized with platinum (W-2 / Pt-5). Feedthrough was fired in the $\mathrm{H}_{2}-\mathrm{Ar}(10-90)$ atmosphere at $1550{ }^{\circ} \mathrm{C}$
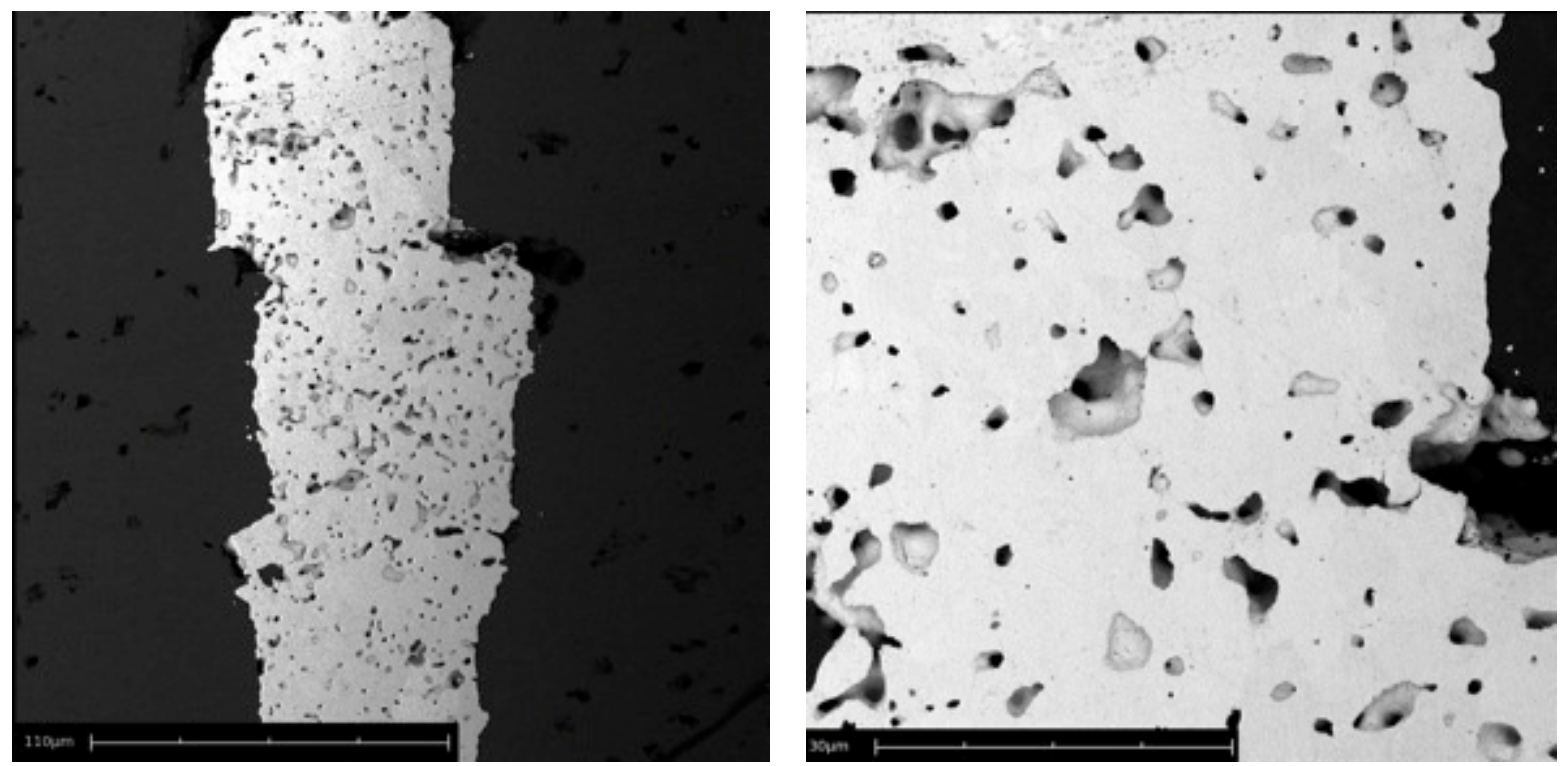

Figure 7-27 Backscatter cross-section image of W-2/Pt-5 (50-50) sample. Feedthrough was fired in the $\mathrm{H}_{2}-\mathrm{Ar}$ (10-90) atmosphere at $1550{ }^{\circ} \mathrm{C}$

Both samples showed very dense structure. In the sample with 50-50 composition, the via was completely filled with tungsten/platinum mixture, while, the 80-20 mixture exhibited detachment from the via. Cross section image in figure 7-22 also shows that the via material has a strong bonding with ceramic and also confirms that there is good adhesion between each layer of tape. The metal mixture in each layer has strong contact with another and this make continuous conductivity possible. The metallization still has a porous structure from the side, but 
it is good enough to satisfy the hermeticity requirement. However, the density of the sample in figure 7-22 is significantly different from the samples showed in figure 7-8, which emphasizes the difference between two furnaces.

\subsection{Tungsten sample}

As mentioned previously, tungsten has a long history in multilayer ceramic industry. Because of that a series of feedthrough were prepared based on the pure tungsten formulation to study the behavior of tungsten in feedthrough structure. The microstructure of the feedthrough with micron sized tungsten particle is shown in figure 7-28.

The tungsten particles exhibit minimal sinter and limited densification. The temperature was not sufficient to promote sintering. However, from the backscatter image, it is clear that the particles joined together with the molten glass between them. Tungsten particles have strong bonding with ceramic. Glass also provides a good matrix structure to join metal particles to each other, and in overall the whole system is functional with a high degree of hermeticity.

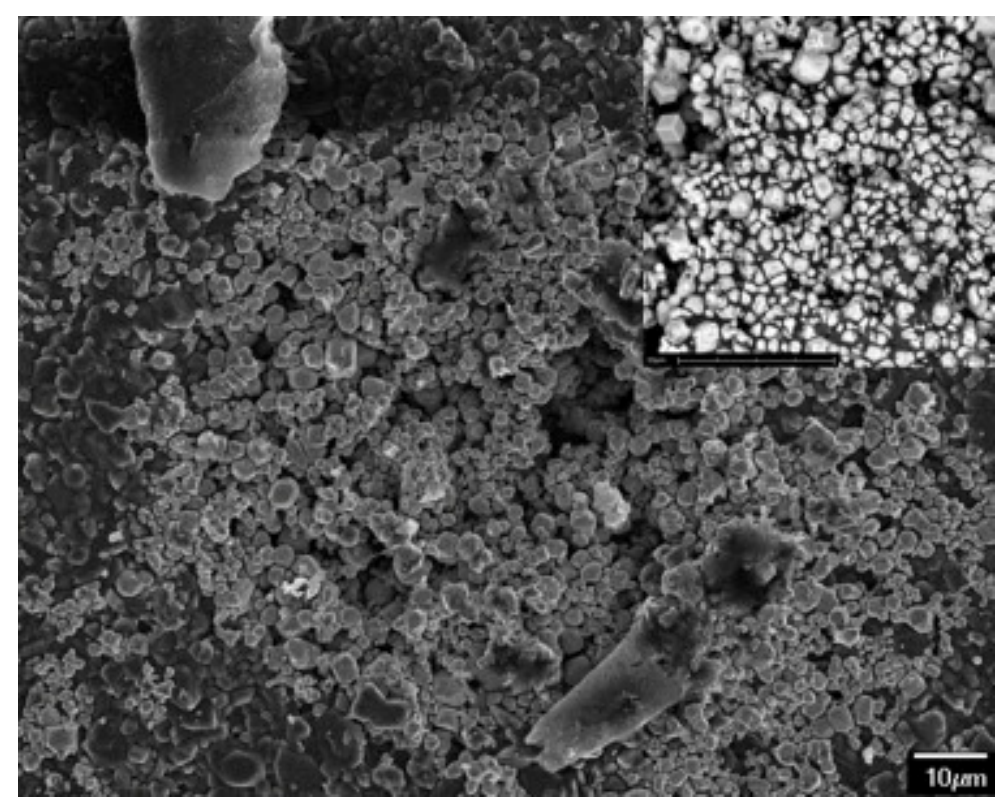

Figure 7-28 SEM image of feedthrough with micron sized tungsten particle (W-1). High magnification image in left corner shows the backscatter image. Feedthrough was fired in the $\mathrm{H}_{2}-\mathrm{Ar}(10-90)$ atmosphere at 1550 
To compare the behavior of the developed tungsten feedthrough, commercially available tungsten paste from AdTech Ceramic Co. (Chattanooga, TN) was used to develop a similar feedthrough. The resultant feedthrough structure is shown in figure 7-29. As it can be seen, the microstructure of the tungsten particle is very similar. Yet the amount of glass particles on top of the via is more than the sample showed in figure 7-28. The ink that was used in the feedthrough shown in figure 7-28 is the pure tungsten without any glass as the filler, while the tungsten ink from AdTech contained glass. High aspect ratio of the via structure creates capillary force to push the light materials on the top surface. This capillary force pushes the glass out of the via and cover the surface of the via,

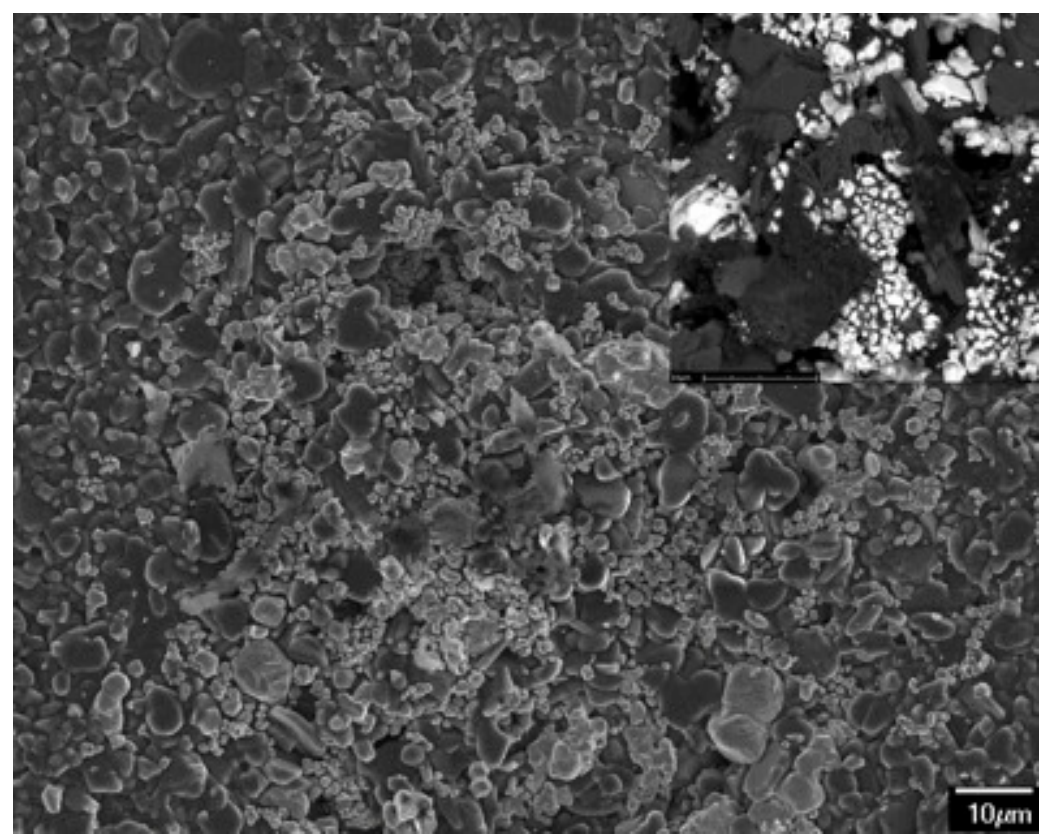

Figure 7-29 SEM image of feedthrough with commerical available tungsten paste from AdTech Cearmic Co. High magnification image in left corner shows the backscatter image. Feedthrough was fired in the $\mathrm{H}_{2}-\mathrm{Ar}$ (10-90) atmosphere at $1550{ }^{\circ} \mathrm{C}$

\subsubsection{Tungsten particle size}

To increase the density of the via material, submicron tungsten particle was used instead of large macron sized particle. The result is shown in figure 7-30. As anticipated, the increased surface free energy of the small particles assisted the sintering in addition to that melted glass 
diffuses into the entire structure of the metal and binds everything together. To achieve the optimum densification and shrinkage of the tungsten, the mixture of the equal ratio of two tungsten particles was used to develop a feedthrough structure.

As it could be seen in figure 7-31, the mixture of two tungsten particles creates an efficient packing to maximize the density and hermeticity of the final feedthrough structure.

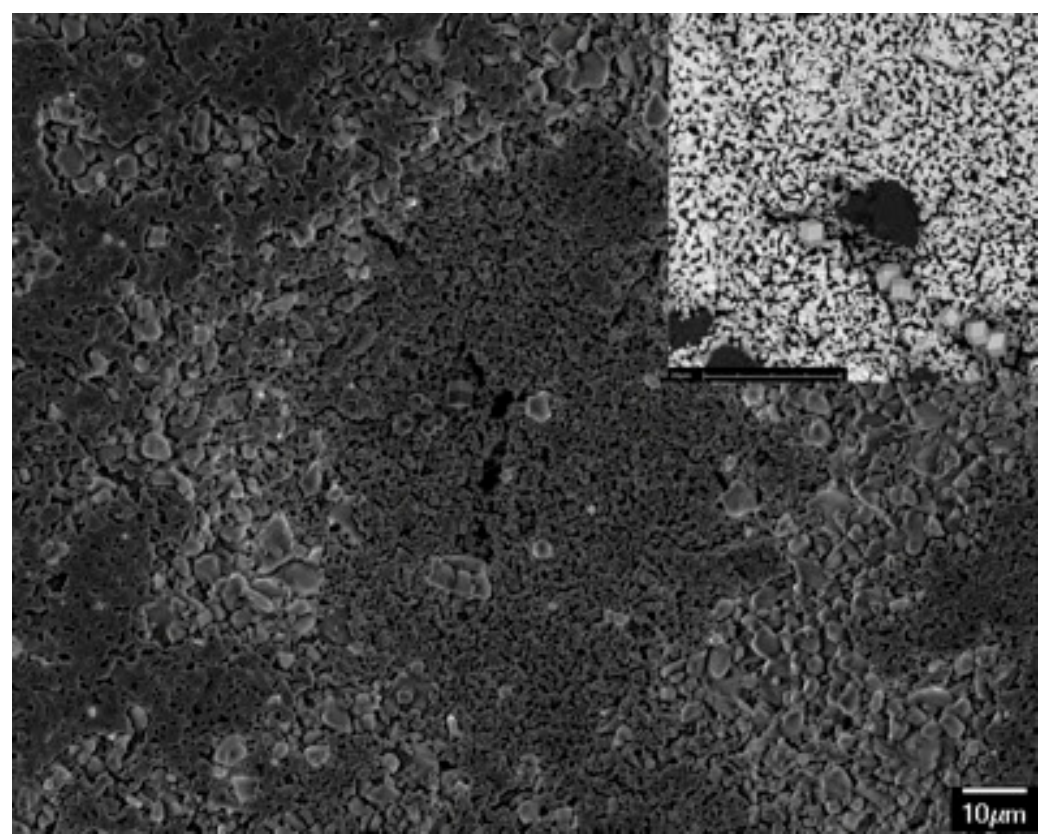

Figure 7-30 SEM image of feedthrough with submicron sized tungsten particle (W-2). High magnification image in left corner shows the backscatter image Feedthrough was fired in the $\mathrm{H}_{2}-\operatorname{Ar}(10-90)$ atmosphere at $1550{ }^{\circ} \mathrm{C}$
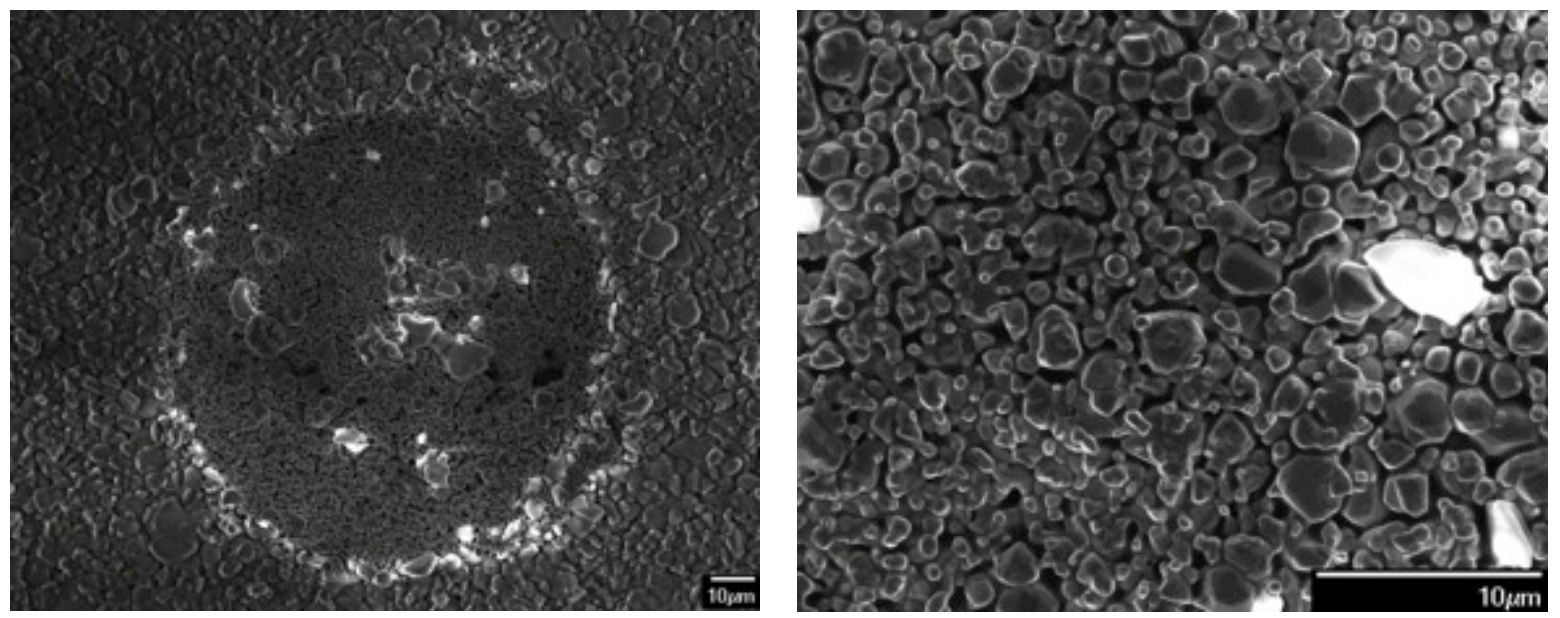

Figure 7-31 SEM image of feedthrough with 50-50 mixture of two tungsten particles. Feedthrough is fired in $10 \% \mathrm{H}_{2}-90 \%$ Ar atmosphere at $1550 \mathrm{c}$ 


\subsubsection{Tungsten-glass sample}

To increase the density of the tungsten particles, $20 \%$ vol. glass was used in the ink formulation. The glass was composed of equal ratio of kaolinite clay and talc, the same glass composition used for the $96 \%$ HTCC alumina tape. The SEM image of the feedthrough is shown in figure 7-32.

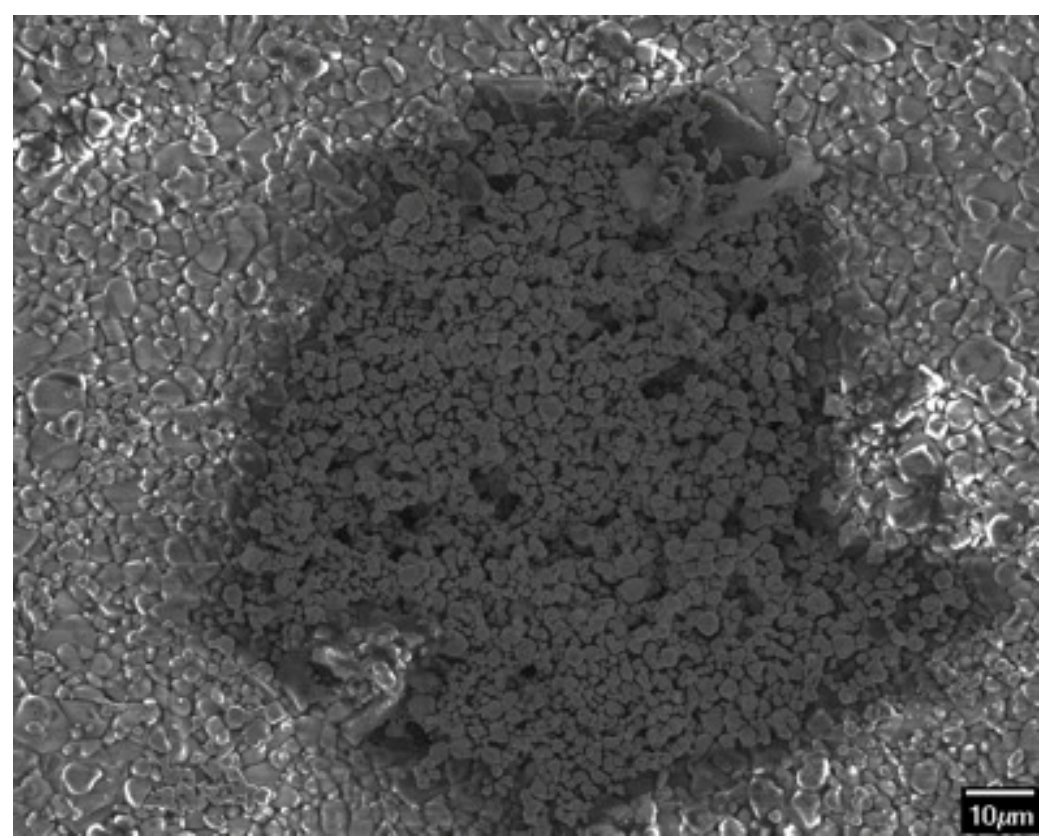

Figure 7-32 SEM image of feedthrough with mixture of micron sized tungsten particles and $20 \%$ glass. Feedthrough was fired in the $\mathrm{H}_{2}-\mathrm{Ar}(10-90)$ atmosphere at $1550{ }^{\circ} \mathrm{C}$

The structure of the sample is very similar to the sample showed in figure 7-27. Glass particles in the ink formulation also melted and diffused between the metal particles. However the structure of the via is not that much different from the sample without glass and the effect of glass in ink formulation is almost negligible. It seems that the amount of glass in the tape formulation is enough to fill the free space between tungsten particles and having more glass in the ink formulation is not necessary. 


\subsection{Composite sample}

To satisfy the requirements of a feedthrough with maximum amount of platinum and hermeticity to $1 \times 10^{-9} \mathrm{cc}-\mathrm{He} / \mathrm{sec}$, a composite sample were prepared. The composite sample consisted of the three tungsten layers for the middle of the structure to obtain the hermeticity, 4 layer of W-2/Pt-5 (50-50) and 2 layers of the W-2/Pt-5 (80-20) for both surfaces. A pure platinum layer is placed on both sides of the feedthrough to satisfy the f requirement for bonding for assembly to the next level of the device (flex circuit and electrode structure). Tungsten layers were made from the equal ratio mixture of two tungsten particles. The cross-section image of such a feedthrough is shown in figure 7-28. It is clear that the materials through the via are completely filled and have good bonding with ceramic. There is good binding between platinum and tungsten layer that provides a continuous electrical conductivity.

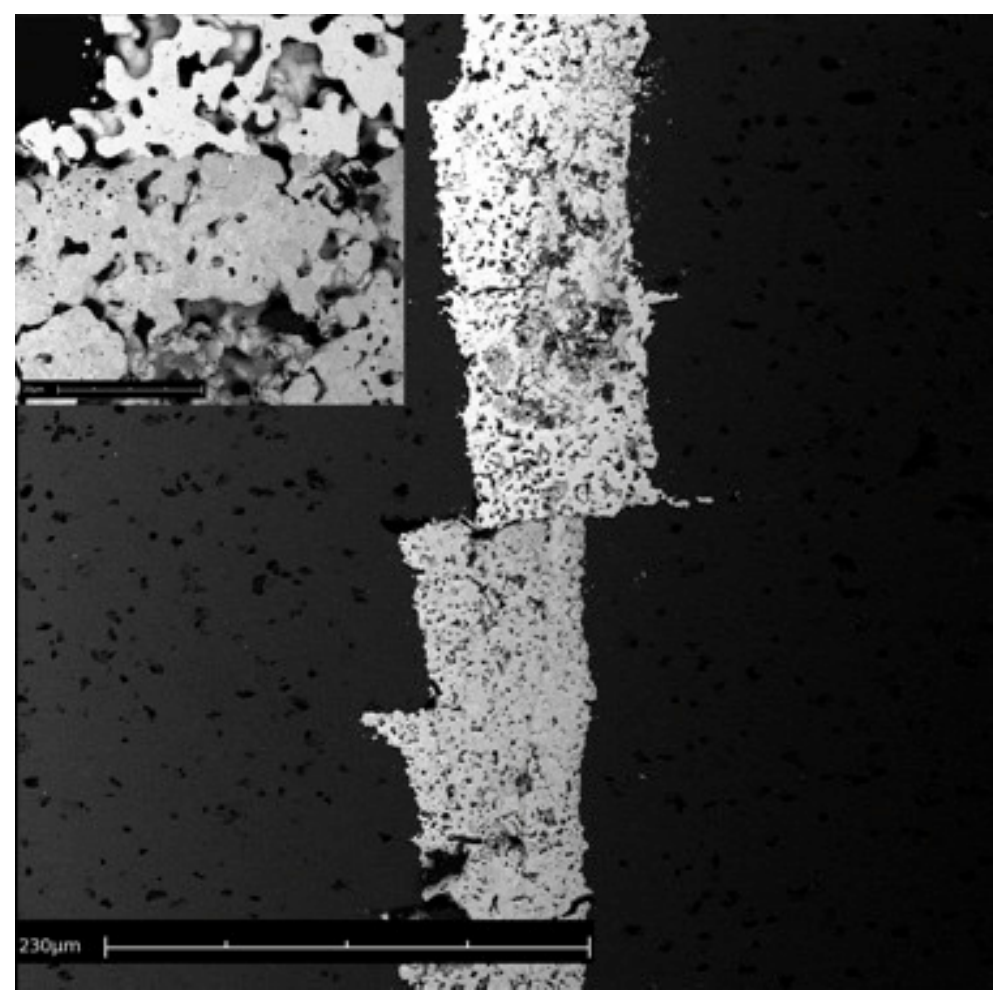

Figure 7-33 Backscatter SEM image of cross-section of the composite sample 


\subsection{Hermeticity measurement}

Due to the high priority of hermeticity requirement for medical industry, as the last step to check the performance of the feedthrough, hermeticity of the different feedthrough structures were measured and shown in table 7-1. For medical application hermeticity of the device should be greater than $1 \times 10^{-9} \mathrm{cc}-\mathrm{He} / \mathrm{sec}$. As it is clear, the tungsten sample, two of the platinum tungsten and composite sample satisfy the hermeticity requirement completely.

Table 7-1 Hermeticty measurements of the different samples

\begin{tabular}{clcc}
\hline Furnace & \multicolumn{1}{c}{ Sample } & Atmosphere & Hermeticity $(\mathrm{cc}-\mathrm{He} / \mathrm{sec})$ \\
\hline \multirow{2}{*}{ GSL -1600X } & Pt-6 & Air & $1.4 \times 10^{-5}$ \\
& Pt-7 & & $5.6 \times 10^{-6}$ \\
& Pt-6 & & $3.5 \times 10^{-7}$ \\
& Pt-6+20\%Pt-4 & & $5.2 \times 10^{-8}$ \\
& Pt-7 & & $>1 \times 10^{-9}$ \\
& Pt-7+20\%Pt-4 & & $>1 \times 10^{-9}$ \\
& Pt-4 & & $5.1 \times 10^{-6}$ \\
& Pt-4+20\%W-2 & $\mathrm{H}_{2}-\mathrm{Ar}(10-90)$ & $1.8 \times 10^{-5}$ \\
& Pt-4+50\%W-2 & & $8.3 \times 10^{-7}$ \\
& Pt-4+80\%W-2 & & $1.5 \times 10^{-7}$ \\
& Pt-5+20\%W-2 & & $2.2 \times 10^{-6}$ \\
& W-1+W-2 & $>1 \times 10^{-9}$ \\
Self-fabricated & Composite Sample & & $>1 \times 10^{-9}$ \\
& Pt-4 & & $>1 \times 10^{-9}$ \\
\hline
\end{tabular}




\section{Platinum/alumina reaction and interaction}

\subsection{Platinum/alumina powder mixture interaction}

Bronger et al. [13] showed that the mixture of platinum and alumina above $1200^{\circ} \mathrm{C}$ in a hydrogen atmosphere for more than 72 hours could react to form $\mathrm{Pt}_{3} \mathrm{Al}$. To study the behavior of platinum/alumina at high temperatures, the mixture with different platinum/alumina ratio were prepared and heated. Different ratios of Pt-4 and n-Alumina, as shown in table 8-1, were used to study the effect of the composition on the reaction product. Each ratio was chosen based on the $\mathrm{Pt} / \mathrm{Al}$ intermetallic compounds of the Pt-Al phase diagram as shown in figure 8-1. Each sample was rapidly heated to $1400{ }^{\circ} \mathrm{C}$ and $1600{ }^{\circ} \mathrm{C}$ and then maintained on that temperature for 8 hours. Experiments were carried out in two different atmospheres (i.e. air and 5\% $\mathrm{H}_{2}-95 \%$ Ar mixture) to study the effect of atmosphere on the reaction.

Table 8-1 Composition of different Pt/Alumina mixture based on different Pt-Al intermetallic compound

\begin{tabular}{llcccc}
\hline Name & & $\mathrm{Pt} 47$ & $\mathrm{Pt65}$ & $\mathrm{Pt79}$ & $\mathrm{Pt92}$ \\
\hline Compound & $\mathrm{Pt}_{\mathrm{x}} \mathrm{Al}_{\mathrm{y}}$ & $\mathrm{Pt}_{5} \mathrm{Al}_{21}$ & $\mathrm{PtAl}_{2}$ & $\mathrm{PtAl}$ & $\mathrm{Pt}_{3} \mathrm{Al}$ \\
\hline \multirow{2}{*}{ Composition } & $\mathrm{Pt}$ wt.\% & 47 & 65 & 79 & 92 \\
& Pt vol.\% & 13.5 & 25 & 40 & 66.5 \\
\hline
\end{tabular}

Low magnification optical images of the samples are shown in figures 8-2 and 8-3. The samples heated in different atmospheres developed two different morphologies. The samples heated in the air atmosphere exhibited a lighter color, which suggest the presence of more aluminum oxide on the surface. As shown in figure 8-2, the surface of Pt92 sample is covered completely with the shiny platinum layer. Pt92 sample fired in hydrogen and air exhibited completely different structure. The sample in hydrogen is completely melted and contracted into 
a spheroid shape, yet the air sample still keeps its structure. This could suggest the idea that hydrogen decreases the melting point of platinum.

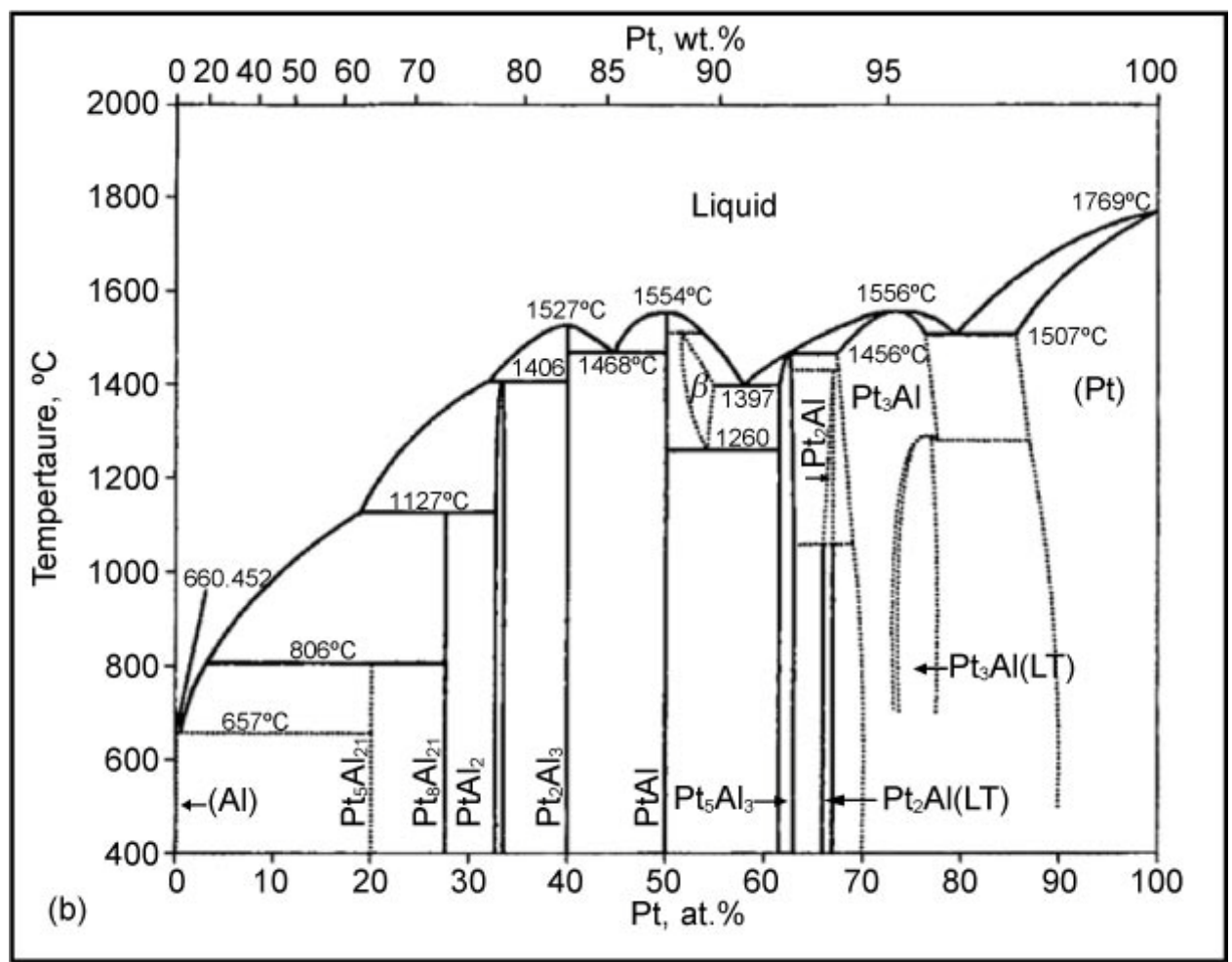

Figure 8-1 Pt-Al Phase diagram

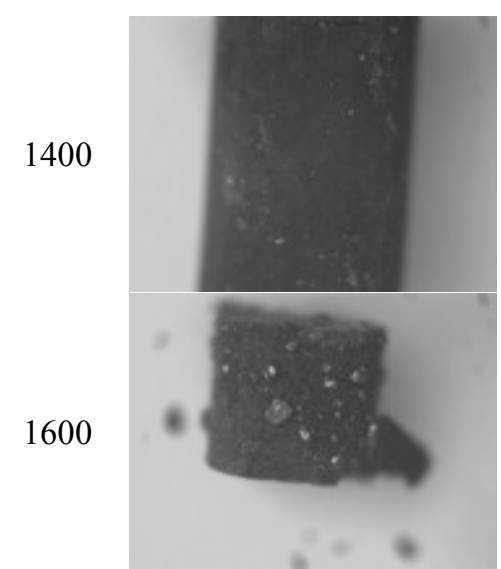

Pt47

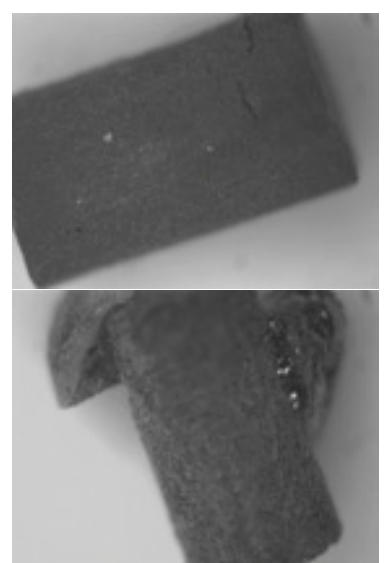

Pt65

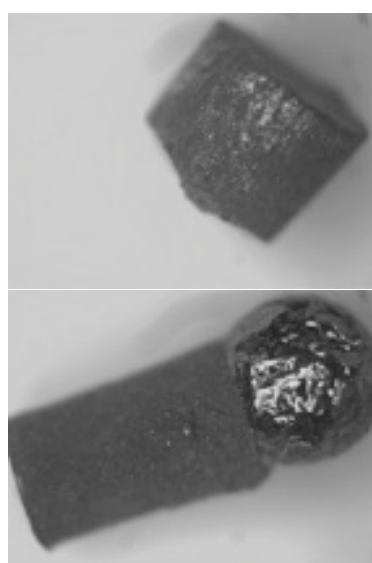

Pt79

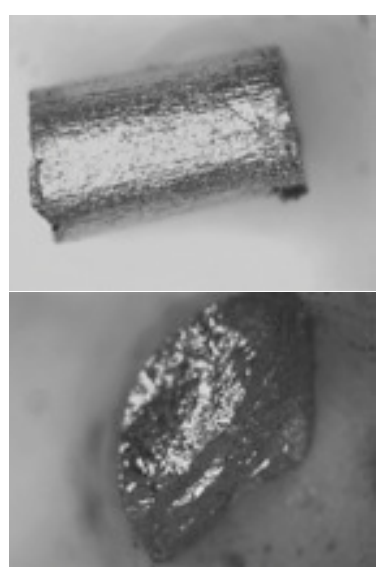

Pt92

Figure 8-2 Optical image of Pt/Alumina samples fired at the $\mathrm{H}_{2}$-Ar (5-95) atmosphere for 8 hour 


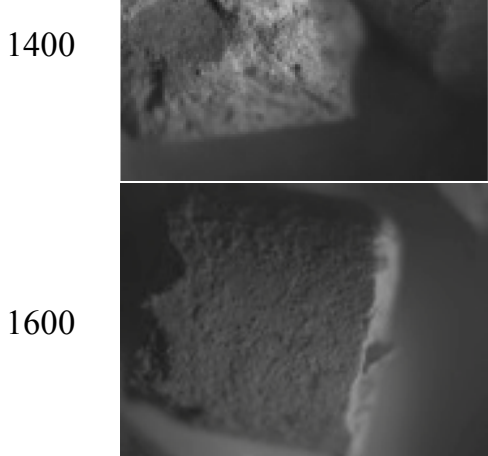

Pt47
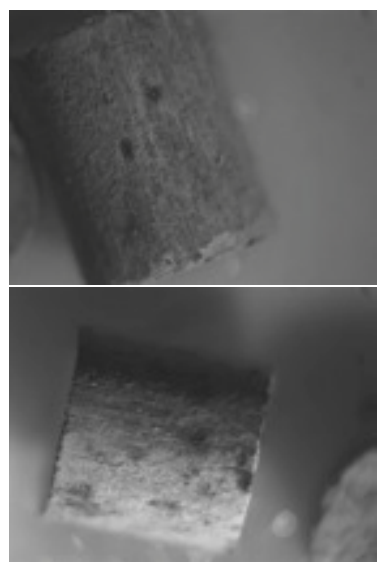

Pt65

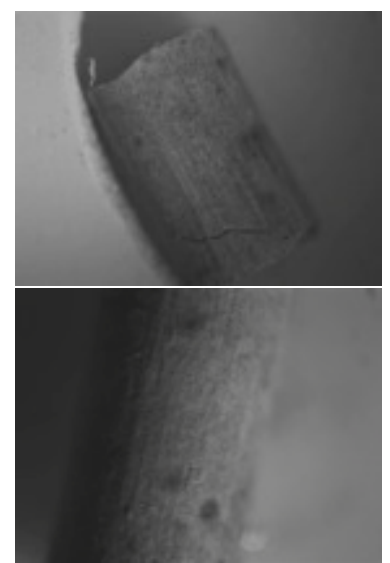

Pt79

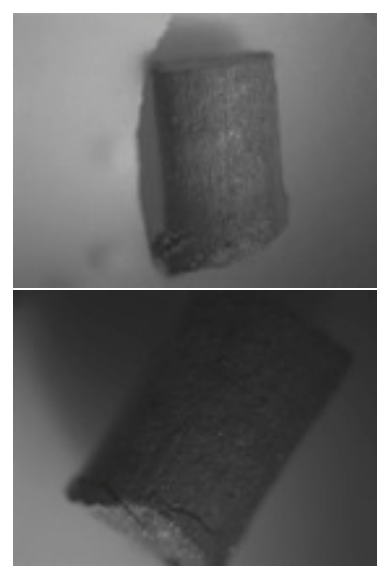

$\mathrm{Pt92}$

Figure 8-3 Optical image of Pt/Alumina samples fired in air for 8 hours

Phase separation happened in some points and platinum moved to the surface of the sample and made spherical metal particle on the surface. An SEM image of a sample with spherical metal surface is shown in figure 8-4.

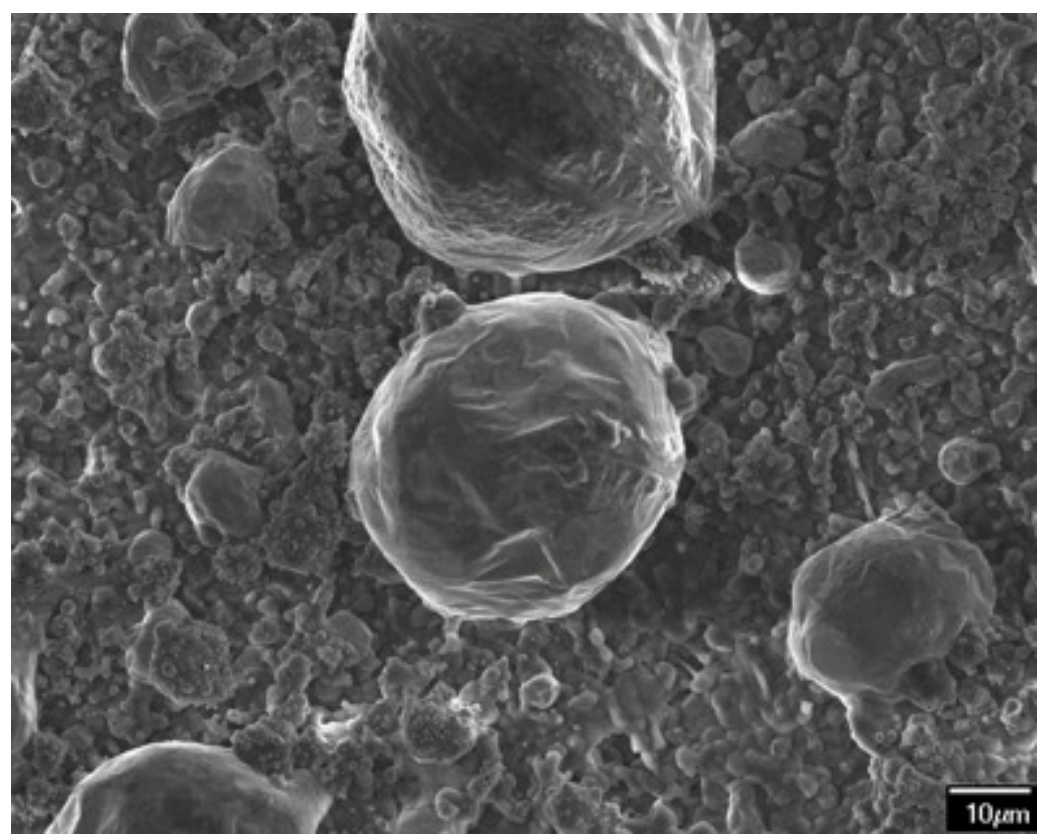

Figure 8-4 SEM image of spherical platinum particles formed on the surface of Pt65 sample at $1600{ }^{\circ} \mathrm{C}$

As expected, the densification of the platinum and platinum compounds are different, and it is shown in figure 8-5 that alumina prevents platinum from sintering. It also decreases the effect of hydrogen as mentioned before. The densification behavior of the different mixtures is shown in figure 8-5. 


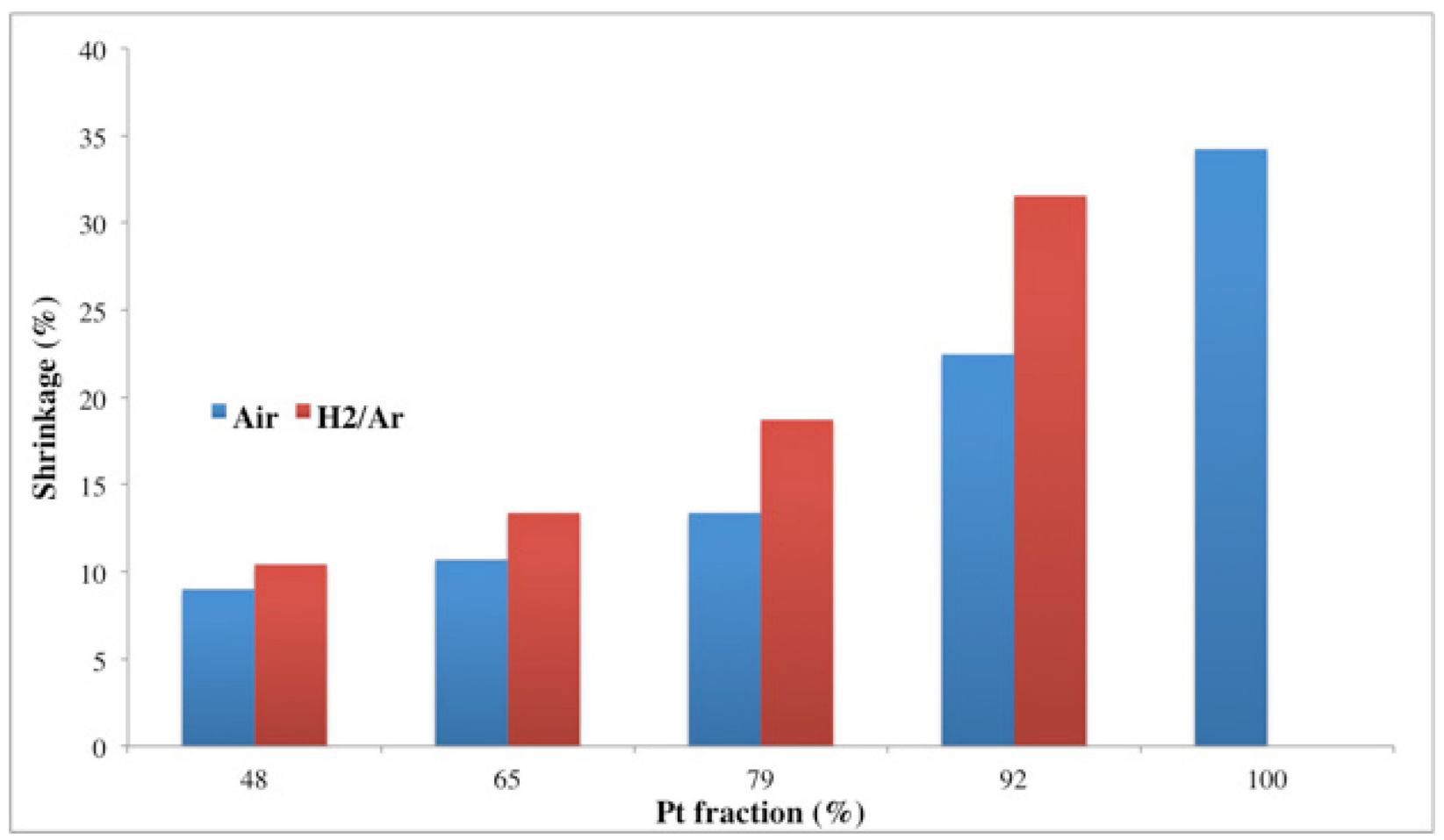

Figure 8-5 Densification behavior of the Pt/Alumina samples in two different atmosphere heated at $1400{ }^{\circ} \mathrm{C}$

$\mathrm{X}$-ray diffraction results of the sample heated in reduced atmosphere are depicted in figure 8-6. Although different ratios of platinum/alumina were used, XRD results indicated that, in all cases, a series of new peaks appeared upon the heating the sample in hydrogen. The first peak was at $2 \theta=10.40^{\circ}$, which corresponds the reaction product between platinum and alumina as $\mathrm{Pt}_{3} \mathrm{Al}$ with the cubic $\mathrm{Cu}_{3} \mathrm{Au}$ structure at high temperature is consistent with the observed peaks. At temperature below $1290^{\circ} \mathrm{C}, \mathrm{Pt}_{3} \mathrm{Al}$ transforms to tetragonal the $\mathrm{Ga}_{3} \mathrm{Pt}$ structure $[149,156]$. It was also reported that it could transform to two other tetragonal structures at lower temperatures [157]. Bronger et al. [13] adopted a $\mathrm{GaPt}_{3}$ tetragonal structure to analyze their experimental data, while the $\mathrm{Cu}_{3} \mathrm{Au}$ structure is in more agreement with our experimental data. The observed $\mathrm{Pt}_{3} \mathrm{Al}$ cubic has a lattice parameter of $3.88 \AA$, which is in good agreement with the literature, $3.876 \AA$ [149]. This could suggest that cubic structure was formed in high temperature, but in the cool down process, slow transformation kinetics limited the transform to the tetragonal structure. 


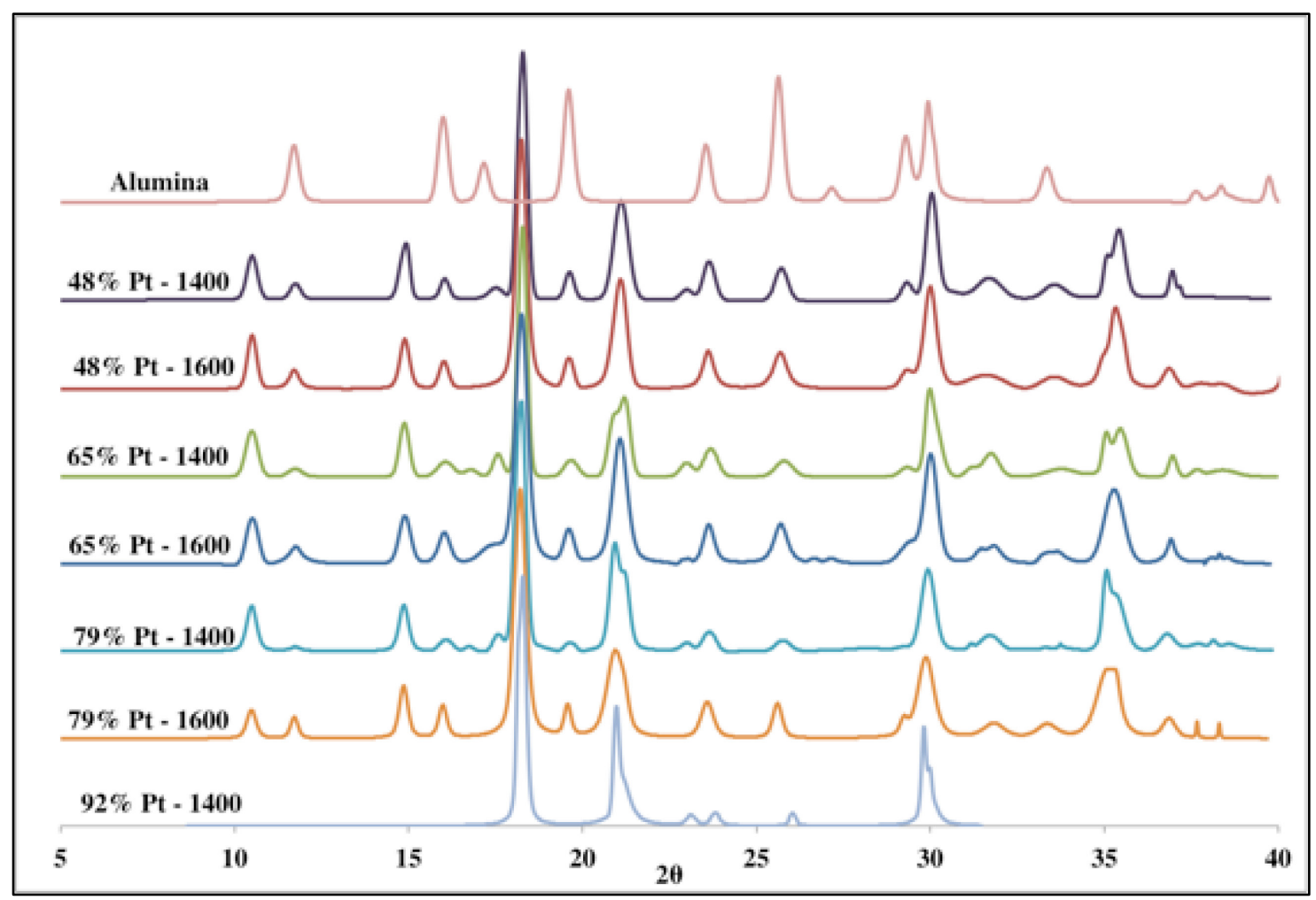

Figure 8-6 X-ray diffraction results of different platinum/alumina samples fired in hydrogen at $1400{ }^{\circ} \mathrm{C}$ and $1600{ }^{\circ} \mathrm{C}$ for 8 hours

The Pt92 sample at both temperatures in the hydrogen atmosphere is completely covered with platinum. XRD of the surface of the Pt92 sample heated at $1400{ }^{\circ} \mathrm{C}$ is also shown in figure $8-6$, which indicates that the surface is almost pure platinum. Pt65 showed the most normal microstructure among all the samples. Because of that, it was chosen for further experiments. The microstructure of the Pt65 sample as-is is shown in figure 8-7.

Figure 8-8 shows the X-ray diffraction of the Pt65 sample heated in air and the reduced atmosphere. It is clear from the XRD result that there is no indication of a new peak appearing in the air firing atmosphere.

Even though the XRD results showed that above $1400{ }^{\circ} \mathrm{C}$, temperature does not have any effect on the reaction, the microstructure of the samples heated at different temperatures are 
completely different. Figure 8-9 showed the microstructures of the Pt65 sample heated in different temperatures and atmospheres.

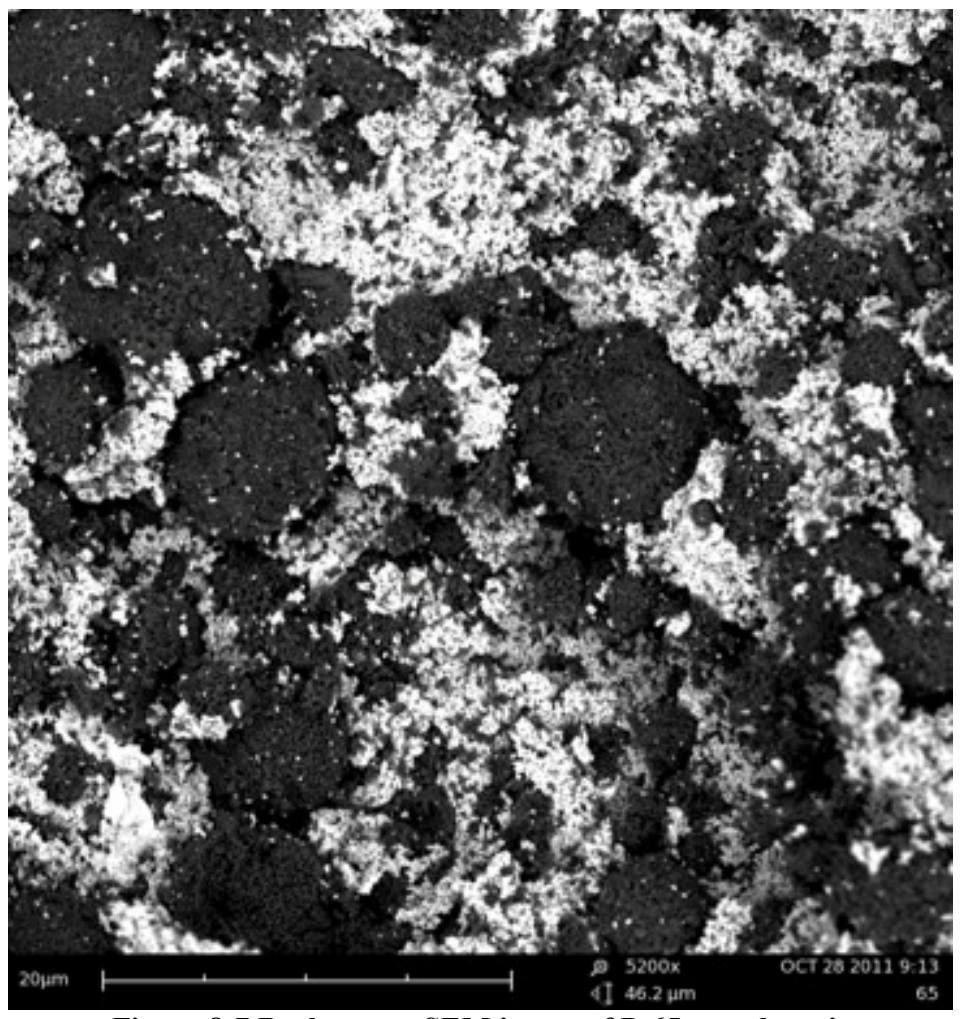

Figure 8-7 Backscatter SEM image of Pt65 sample as-is

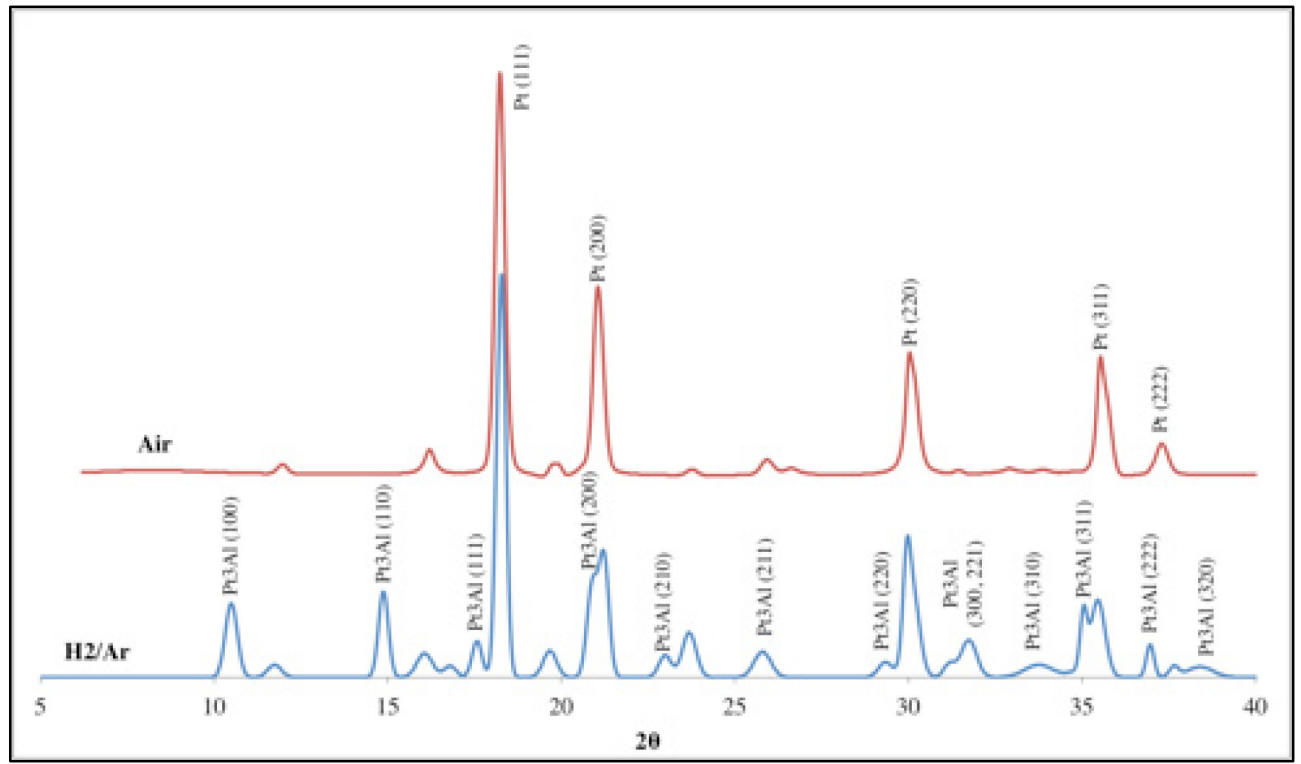

Figure 8-8 X-ray diffraction results of Pt65 sample fired in hydrogen and air at $1400 \mathrm{C}$ for 8 hours 
In general, the n-Alumina powder is received as the agglomerated particles and dry ball milling has minimal effect on its structure, shown figure 8-7. Upon heating the sample in air up to $1400{ }^{\circ} \mathrm{C}$, platinum particles grow without any interaction with alumina. Increasing the temperature up to $1600{ }^{\circ} \mathrm{C}$ only makes the platinum growth more, which means that platinum particles stick together, separate from the platinum/alumina mixture, and alumina granulates fill almost the entire surface. Because of this, the surface of the samples heated in an air atmosphere in figure 8-3, seemed to be filled with alumina and with an unsintered ceramic texture.

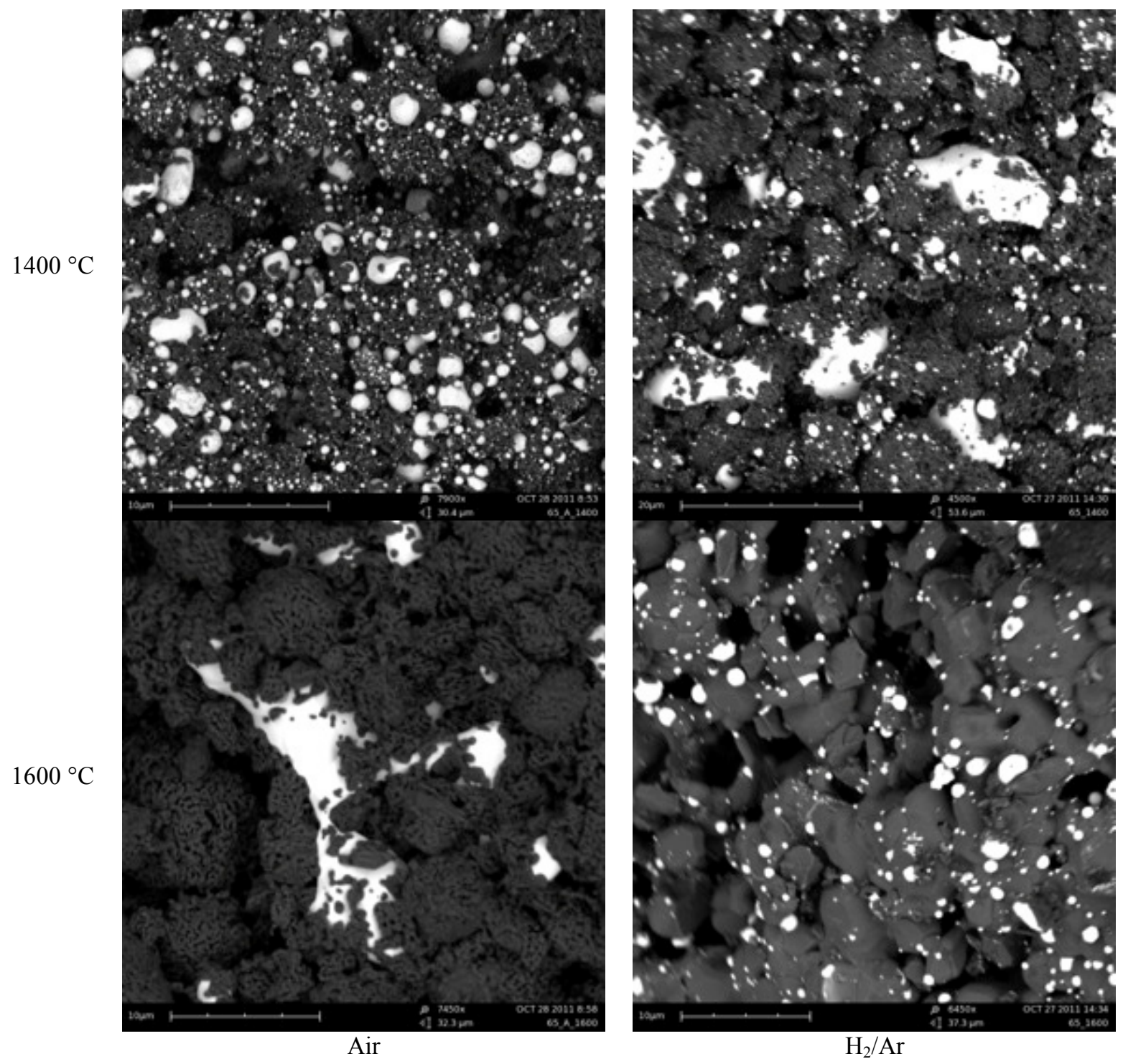

Figure 8-9 Backscatter SEM image of Pt65 sample fired in different temperatures and atmospheres 
Heating the sample in hydrogen helps the platinum particles growth. The size of the platinum particles heated in $1400^{\circ} \mathrm{C}$ is similar in size to the platinum particles heated in air at $1600{ }^{\circ} \mathrm{C}$. Yet, the interaction of platinum with alumina is not similar to the air fired sample, possibly due to a $\mathrm{Pt}-\mathrm{Al}$ compound was formation in the reduced atmosphere. The most important aspect of this mixture happened upon heating the sample in hydrogen up to $1600{ }^{\circ} \mathrm{C}$. As is shown in figures 8-10 and 8-11, the agglomerated alumina particles grow and transform to a large grained structure. Platinum particles were melted and the already-grown alumina particles were attached to their surface. In some areas, alumina acts as the coating, migrate and cover the surface of the platinum, as shown in figure 8-11.

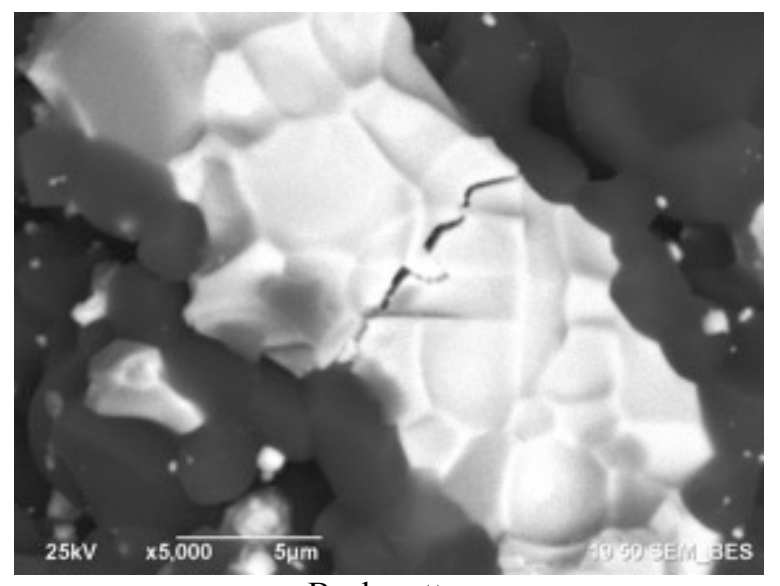

Backscatter

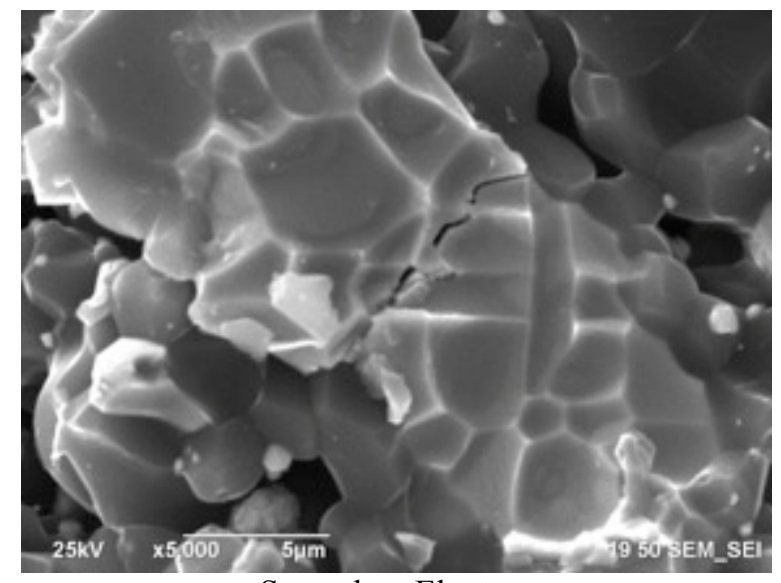

Secondary Electron

Figure 8-10 SEM image of Pt65 sample fired in $\mathrm{H}_{2}$-Ar (5-95) atmosphere for 8 hours at $1600{ }^{\circ} \mathrm{C}$

\subsubsection{Temperature of the reaction}

Pt65 sample were heated up to different temperatures to determine the minimum reaction temperature. Samples were heated at the rate of $10{ }^{\circ} \mathrm{C} / \mathrm{min}$ to the desired temperatures and maintained for one hour. A high hydrogen concentration, 25\% $\mathrm{H} 2-75 \% \mathrm{Ar}$, was used in this experiment. The XRD results are shown in figure 8-12, and it is obvious that reaction starts to occur after $1350{ }^{\circ} \mathrm{C}$ is reached. The intensity of the $\mathrm{Pt}_{3} \mathrm{Al}$ peaks at $1350{ }^{\circ} \mathrm{C}$ is very low, but it still indicates the occurrence of the reaction between platinum and alumina. 


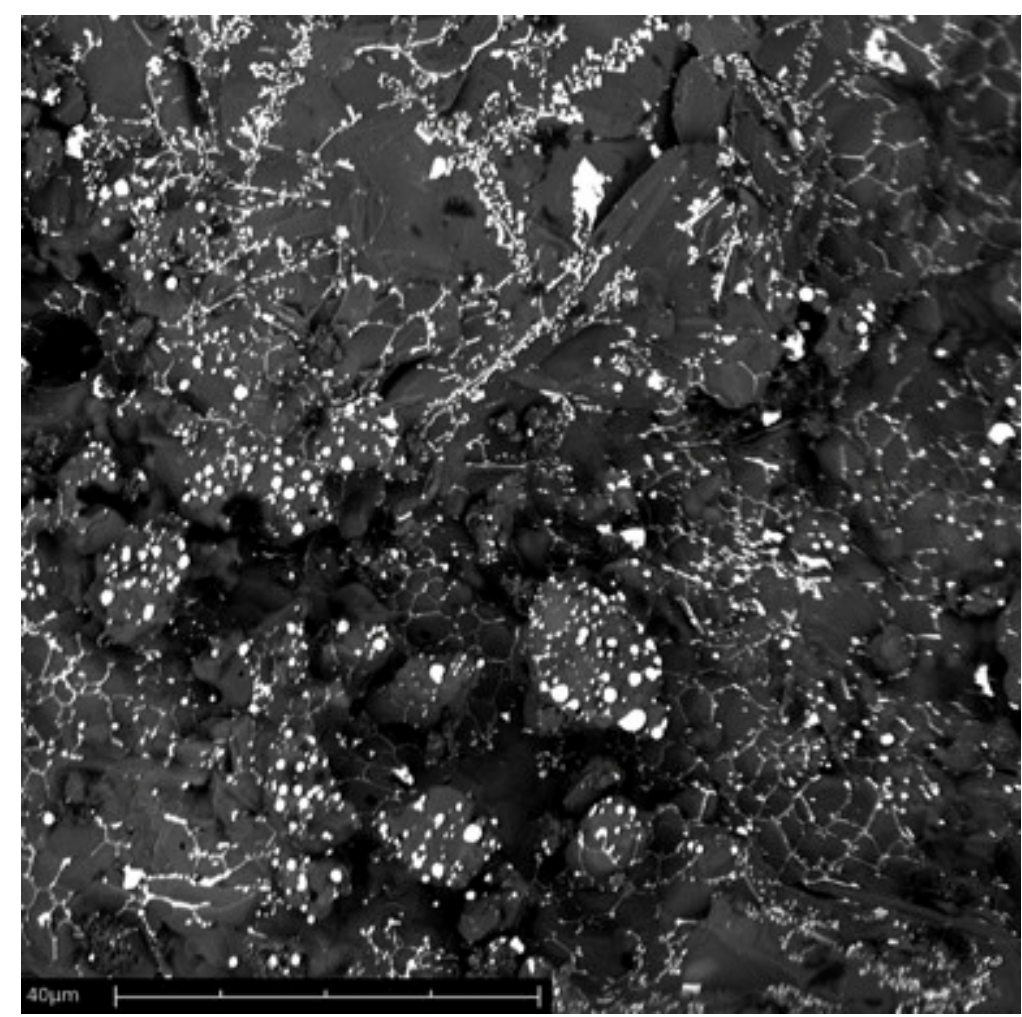

Figure 8-11 Microstructure of the alumina coating on the surface of the platinum in the Pt65 sample heated in hydrogen at $1600 \mathrm{C}$

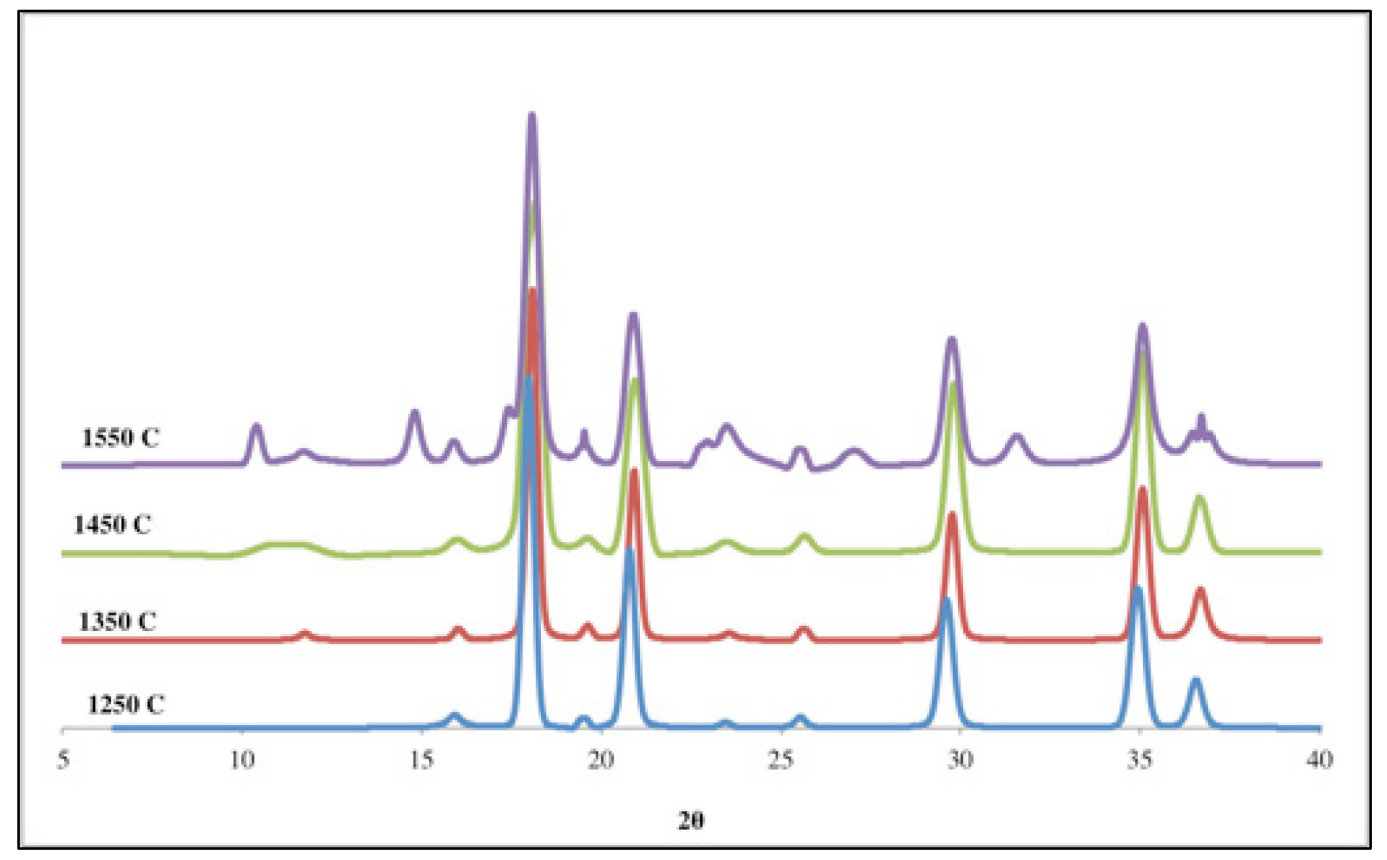

Figure 8-12 X-ray diffraction results of Pt65 sample heated in H2-Ar (25-75) atmosphere at different temperatures 
From figure 8-13, the microstructure of the Pt65 sample exhibit minimal change before the temperature reaches $1550{ }^{\circ} \mathrm{C}$. Nano alumina particles is sintered and transfer to a large grained structure as the temperature reaches $1550{ }^{\circ} \mathrm{C}$.

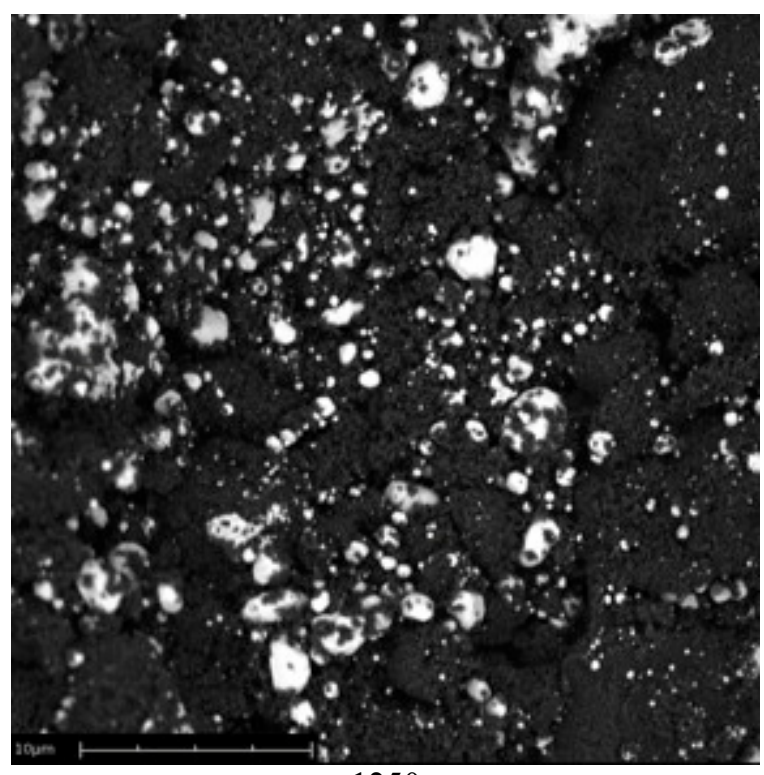

1250

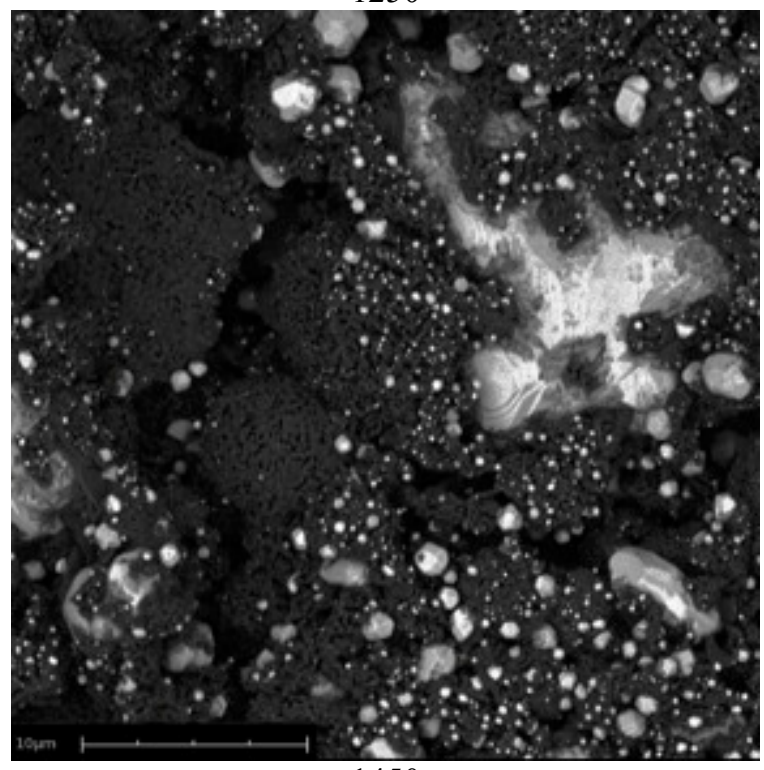

1450

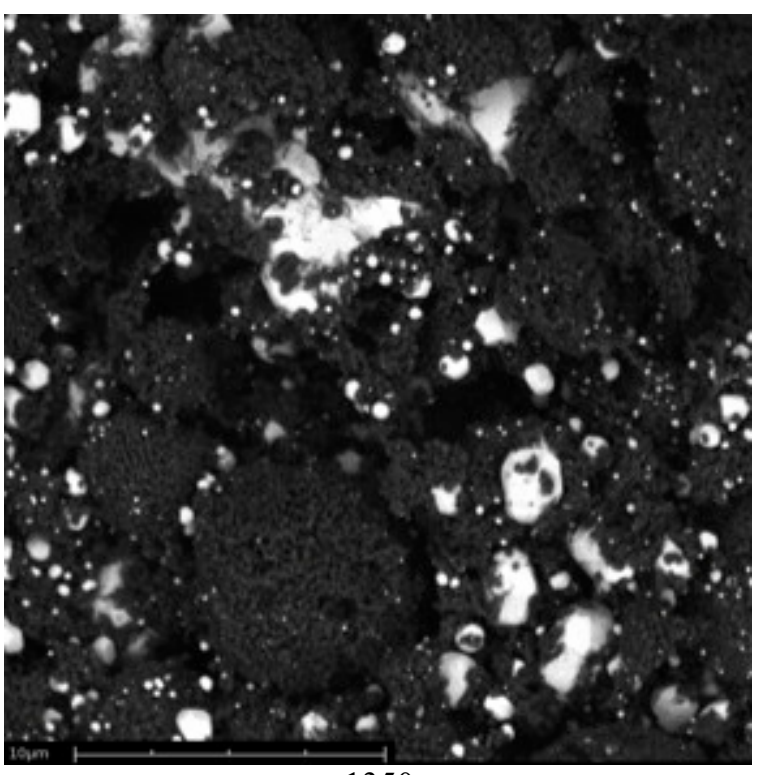

1350

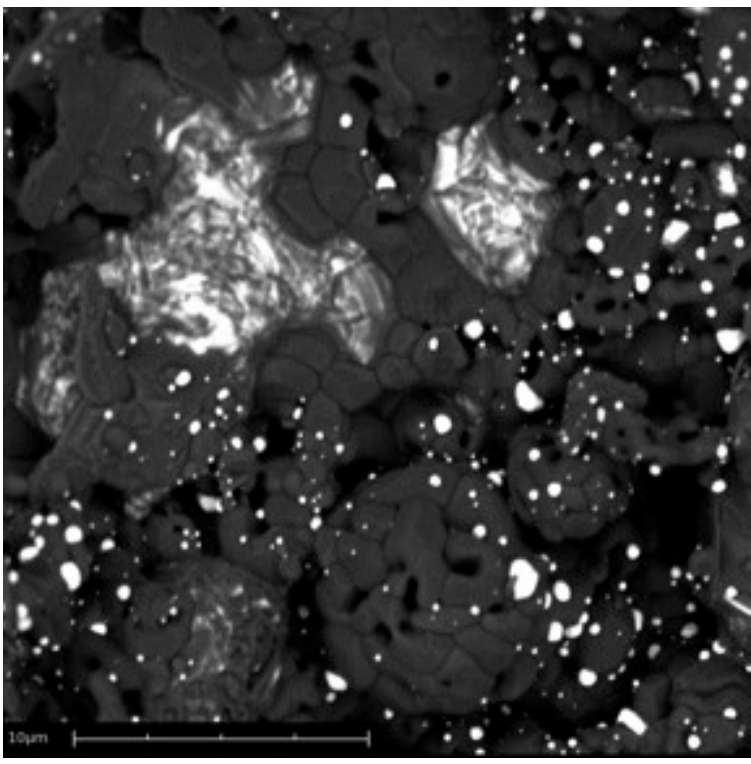

1550

Figure 8-13 Backscatter image of Pt65 sample fired at different temeprature at the H2-Ar (25-75) atmosphere. Samples heated with $10{ }^{\circ} \mathrm{C} / \mathrm{min}$ and kept on that temperature for 1 hour

For a better understanding the platinum and alumina reaction, high temperature X-ray

diffraction technique were used. In this way, the Pt65 sample was heated in a self- 
fabricated cell with a graphite heater [141]. In situ X-ray diffraction data of the sample at high temperatures were recorded at the CHESS synchrotron facility at Cornell University. The XRD results at different temperatures are shown in figure 8-13. It should be noted that graphite was used as the reducing agent to study the effect of temperature on the platinum-alumina reaction. The sample maintained at each temperature for 5 minutes and then X-ray data recorded. Due to the short amount of reaction time, the intensity of the observed diffraction peaks in comparison to platinum is negligible. Because of that only the portion of the diffraction pattern with more emphasis on the reaction product is shown.

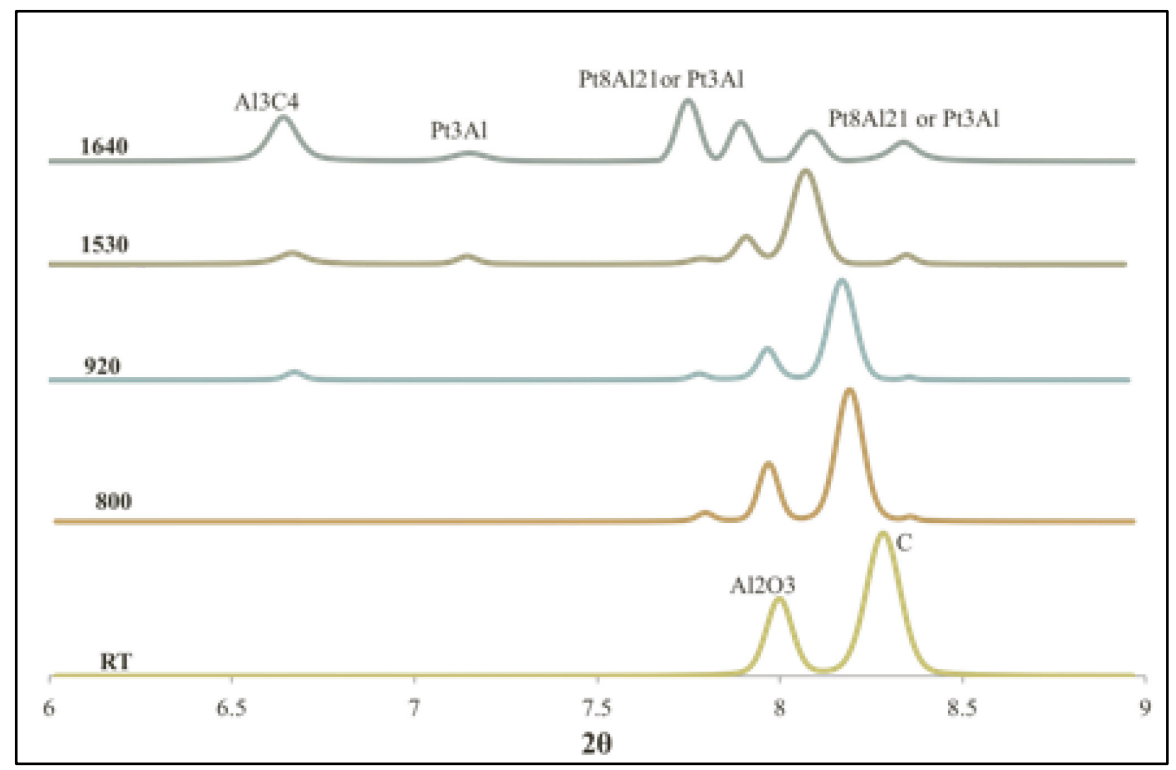

Figure 8-14 In situ X-ray diffraction results of Pt65 sample in different temperature

The first two peaks in $2 \theta=7.77^{\circ}$ and $8.35^{\circ}$ appeared at the temperature about $800^{\circ} \mathrm{K}$. Those peaks are related to tetragonal $\mathrm{Ir}_{3} \mathrm{Si}$ structure. $\mathrm{Pt}_{3} \mathrm{Al}$ in temperature lower than $400{ }^{\circ} \mathrm{C}$ has tetragonal crystal structure [157]. $\mathrm{Pt}_{8} \mathrm{Al}_{21}$ also has the same crystal structure [149]. By increasing the temperature up to $920^{\circ} \mathrm{K}$, another peak appeared at $2 \theta=6.65^{\circ}$. That peak is attributed to the formation of rombohedral structure of aluminum carbide [158]. In the temperature above $1500^{\circ} \mathrm{K}$, a new peak appeared at $2 \theta=7.15^{\circ}$, which corresponds to the cubic $\mathrm{Pt}_{3} \mathrm{Al}$ structure. The 
$\mathrm{Ir}_{3} \mathrm{Si}$ tetragonal structure could transform to cubic $\mathrm{Cu}_{3} \mathrm{Al}$ easily. The $\mathrm{Pt}_{3} \mathrm{Al}$ was formed with the tetragonal structure at low temperature and the crystal structure transformed to the cubic structure at high temperatures. From the results showed in figure 8-6, the equilibrium structure of the $\mathrm{Pt}_{3} \mathrm{Al}$ is cubic, with sufficient amount of time and hydrogen concentration, the tetragonal structure transforms completely to the cubic structure. On the other hand, Zhong et al. [15] report the formation of $\mathrm{Pt}_{8} \mathrm{Al}_{21}$ in the $\mathrm{H}_{2}-\mathrm{Ar}(10-90)$ atmosphere. It is reasonable to assume the tetragonal $\mathrm{Pt}_{8} \mathrm{Al}_{21}$ was formed at low temperature and then transferred to the cubic $\mathrm{Pt}_{3} \mathrm{Al}$ at higher temperatures. However, Zhong et al. [15] used TEM diffraction to confirm the formation of $\mathrm{Pt}_{8} \mathrm{Al}_{21}$, which could not rule out the formation of the tetragonal $\mathrm{Pt}_{3} \mathrm{Al}$ instead of $\mathrm{Pt}_{8} \mathrm{Al}_{21}$. Phase transformation of the tetragonal $\mathrm{Pt}_{3} \mathrm{Al}$ to cubic $\mathrm{Pt}_{3} \mathrm{Al}$ at high temperature is thermodynamically favorable process [157], while $\mathrm{Pt}_{8} \mathrm{Al}_{21}$ to $\mathrm{Pt}_{3} \mathrm{Al}$ transformation is not. It could be concluded that the formation of tetragonal $\mathrm{Pt}_{3} \mathrm{Al}$ at low temperatures and then phase transformation at higher temperatures is more reasonable explanation for the reaction of platinum and aluminum oxide.

\subsection{Platinum thick film interaction with alumina}

A $10 \mu \mathrm{m}$ thick film of Pt-4 were applied on the $99.6 \%$ alumina substrate to study the effect of platinum on microstructure of alumina at high temperatures. The sample was heated up to $1550{ }^{\circ} \mathrm{C}$ in the $\mathrm{H}_{2}-\mathrm{Ar}(10-90)$ atmosphere. The microstructure of the fired sample is shown in figures 8-15 to 8-17.

Platinum powder started to coalescence and form particles with different sizes at high temperatures. It is clear from figure $8-15$, that platinum is not uniformly distributed, which could be due to the thick film thickness distribution. It seems that upon heating some of the platinum 
particles were covered with a layer of ceramic. This happens mostly for the larger particles, which are, by average, larger than $10 \mu \mathrm{m}$ in diameter. SEM images of the platinum with different sizes are shown in figures 8-16 and 8-17. Backscatter top view of the particles in figure 8-15 shown that almost entire platinum surface is covered with ceramic. In addition to that, the side view secondary electron image shown in figure 8-16 also showed that altering the particle size altered the microstructure of the ceramic coating

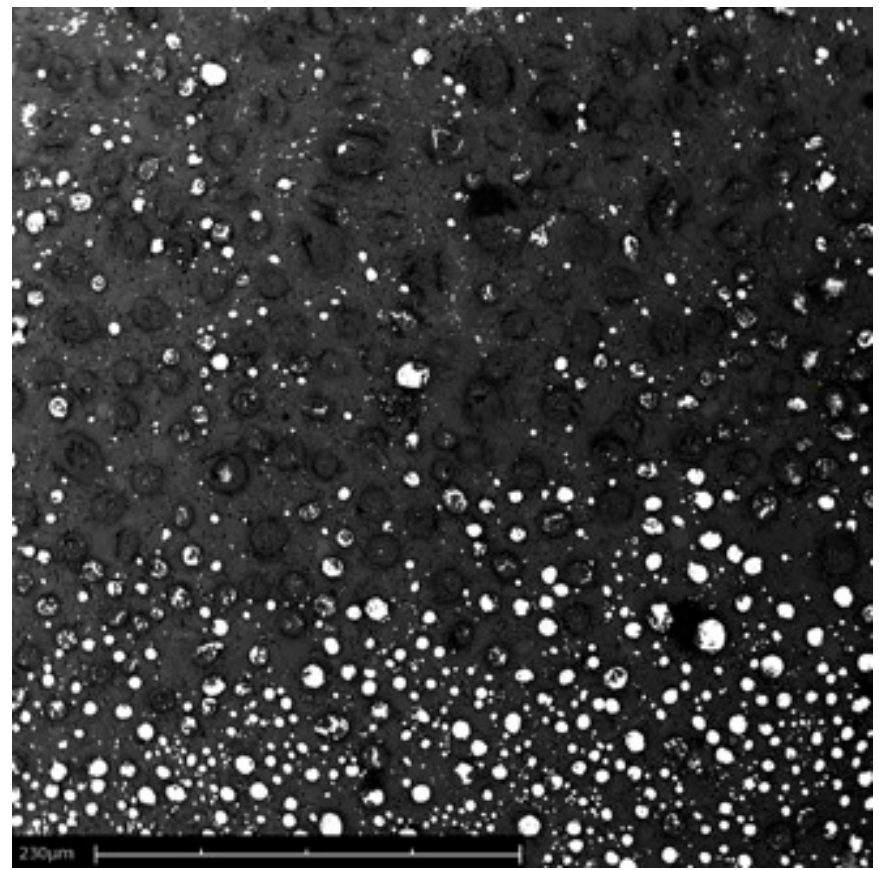

Figure 8-15 Backscatter SEM image of thick film of platinum on 99.6\% alumina substrate fired at $1550{ }^{\circ} \mathrm{C}$ in $\mathrm{H}_{2}-\mathrm{Ar}(10-$ 90) atmosphere for 3 hour

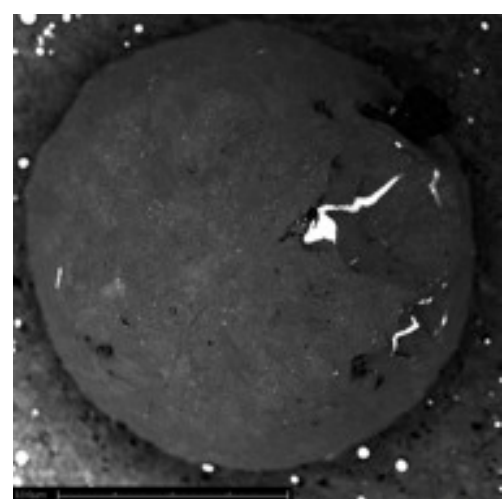

a

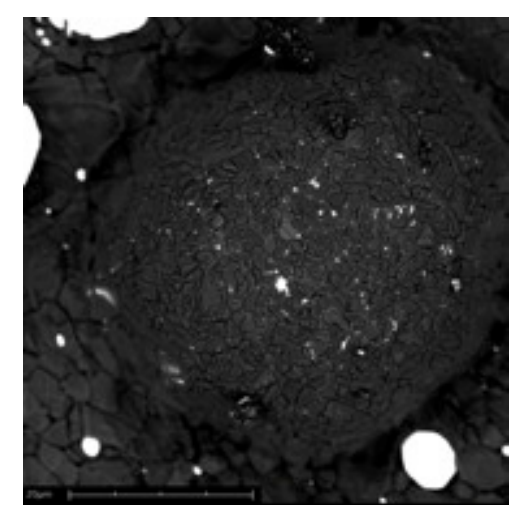

b

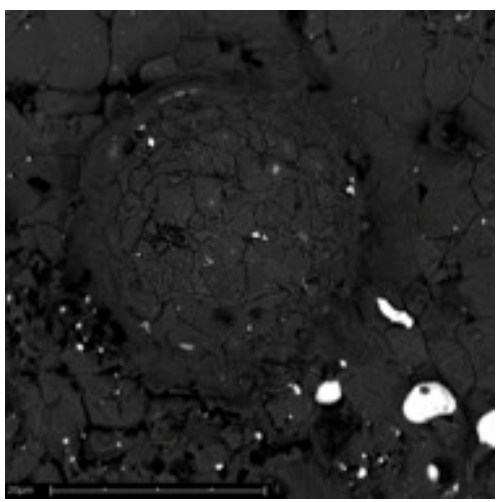

$\mathrm{c}$

Figure 8-16 Backscatter SEM image of thick film of platinum on $99.6 \%$ alumina substrate fired at $1550{ }^{\circ} \mathrm{C}$ in $\mathrm{H}_{2}-\mathrm{Ar}(10-$ 90) atmosphere for 3 hours 
In figure 8-16, particles (a), (b) and (c) are 195, 42 and $27 \mu \mathrm{m}$ in diameter respectively. In figure 8-17, particles (a) and (b) are 90 and $17 \mu \mathrm{m}$. It is clear, increasing the particle size increases the order structure of the alumina coating. Alumina coating, which has covered the surface of particle 8.17 (b), is very rough. While for the particle 8-17 (a), the coating has a very fine structure. The same is true for the particles shown in figure 8-16.

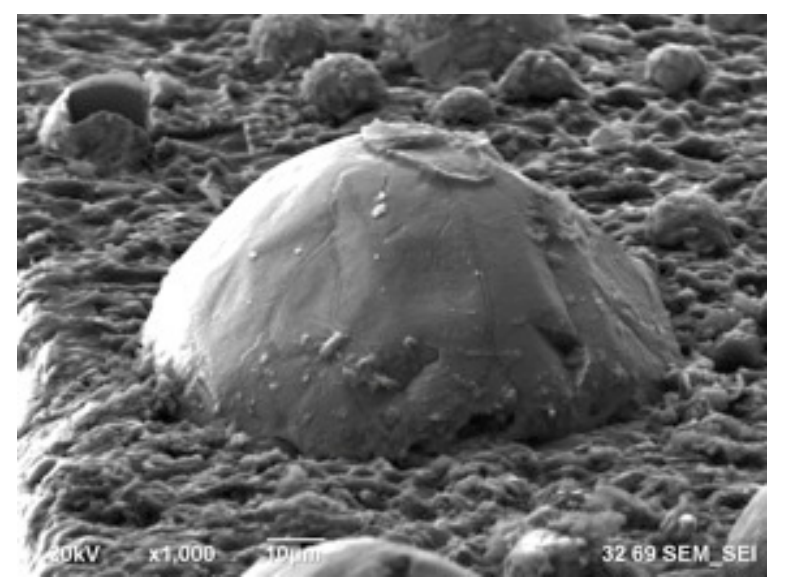

a

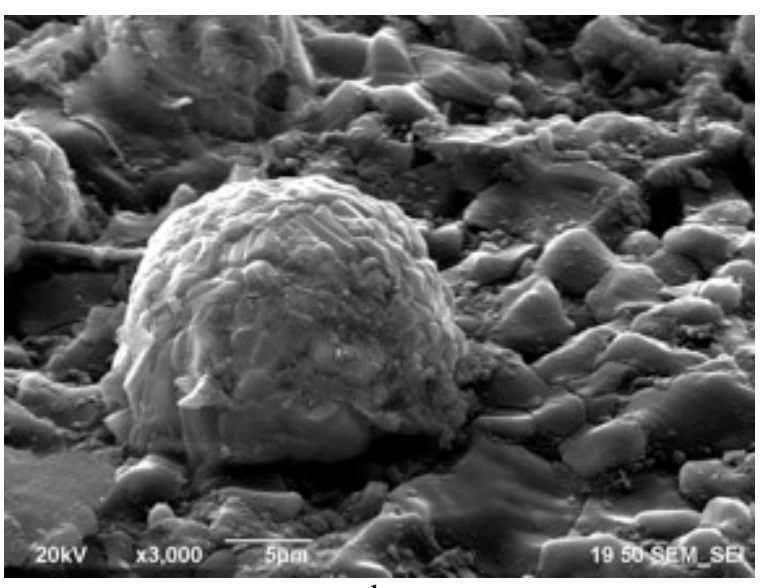

b

Figure 8-17 SEM image of thick film of platinum on $99.6 \%$ alumina substrate fired at $1550{ }^{\circ} \mathrm{C}$ in the $\mathrm{H2}-\mathrm{Ar}(10-90)$ atmosphere for 3 hours

As discussed in section 2.3.2, because of difference in the surface tension and work function of metal and oxide, $\mathrm{TiO}_{2}$ could migrate on the surface of the platinum particle and encapsulated it; while in normal condition it is not possible for aluminum oxide to cover the platinum [26]. However, in a reduced atmosphere at high temperatures, alumina migrates over the surface of the platinum and covers it. Alumina can migrate over the platinum surface with either a mechanism of atomic or particulate migration. Baker et al. [81, 82] stated the migration mechanism is atomic migration when the interaction is strong but changes to the particle migration when the interaction is weak. Straguzzi et al. [83] showed that the interaction force depends mostly on the surface area, which determines the binding points. For small surface area particles the interaction is probably weak and the resistance upon migration is low, so they can 
migrate on the surface of oxide and sinter upon collision. While for high surface area particles, the growth can occur only through the atomic migration. In case of the particles shown in figures 8-15 to 8-17, alumina migrated with particle migration mechanism. Those particles are formed from the agglomeration of pre-sintered submicron platinum Pt-4 particle. They have low surface area, which decreases the interaction force and make it possible for the alumina particles to migrate.

The alteration of the microstructure of alumina in the temperature above $1500^{\circ} \mathrm{C}$ needs to be explained in another way. As observed in figure 7-5, alumina could be absorbed from the sidewalls of the via and move to the top surface. However in case of particles showed in figures 8-16 and 8-17 it is not clear that alumina was absorbed in to the platinum and moved to the top surface of the particle. As observed in figure 7-9, alumina starts to migrate from the surrounding area and covered the platinum. It could be said it is similar to the encapsulation process of platinum with titanium dioxide as shown in figure 2-7. Particles showed in figures 8-16 and 8-17 are formed from the coalescence of small particles. This enables the alumina particles to move through the platinum particle. Increasing the platinum particle size increases the distance that alumina needs to travel to reach the top surface. Hence, the larger portion of alumina dissolved, the finer structure of the final coating. It was showed in the Pt-Al phase diagram $[149,159]$ that the $\mathrm{Pt} / \mathrm{Pt}_{3} \mathrm{Al}$ mixture become liquid at temperature above $1500{ }^{\circ} \mathrm{C}$. Liquid mixture of $\mathrm{Pt} / \mathrm{Pt}_{3} \mathrm{Al}$ creates an appropriate medium for the ceramic particles migration and increases the possibility of alumina absorption and migration. The proposed mechanism is very similar to glass migration mechanism [66]. The microstructure of the compound observed in figures 8-10 and 8-11 could be explained in this way. Platinum helps alumina to grow and because of that the whole surface of the platinum particles are covered with alumina. 


\subsection{Platinum thin film interaction with alumina}

To study the behavior of platinum thin film on alumina, thin film of platinum with thickness of

50 and $200 \mathrm{~nm}$ was deposited on 99.6 alumina substrates. At the first step, the samples were

rapidly heated to $1400{ }^{\circ} \mathrm{C}$ and $1550{ }^{\circ} \mathrm{C}$ in two different hydrogen concentrations and maintained for an hour. The structures of the $50 \mathrm{~nm}$ samples are shown in figure 8-18.

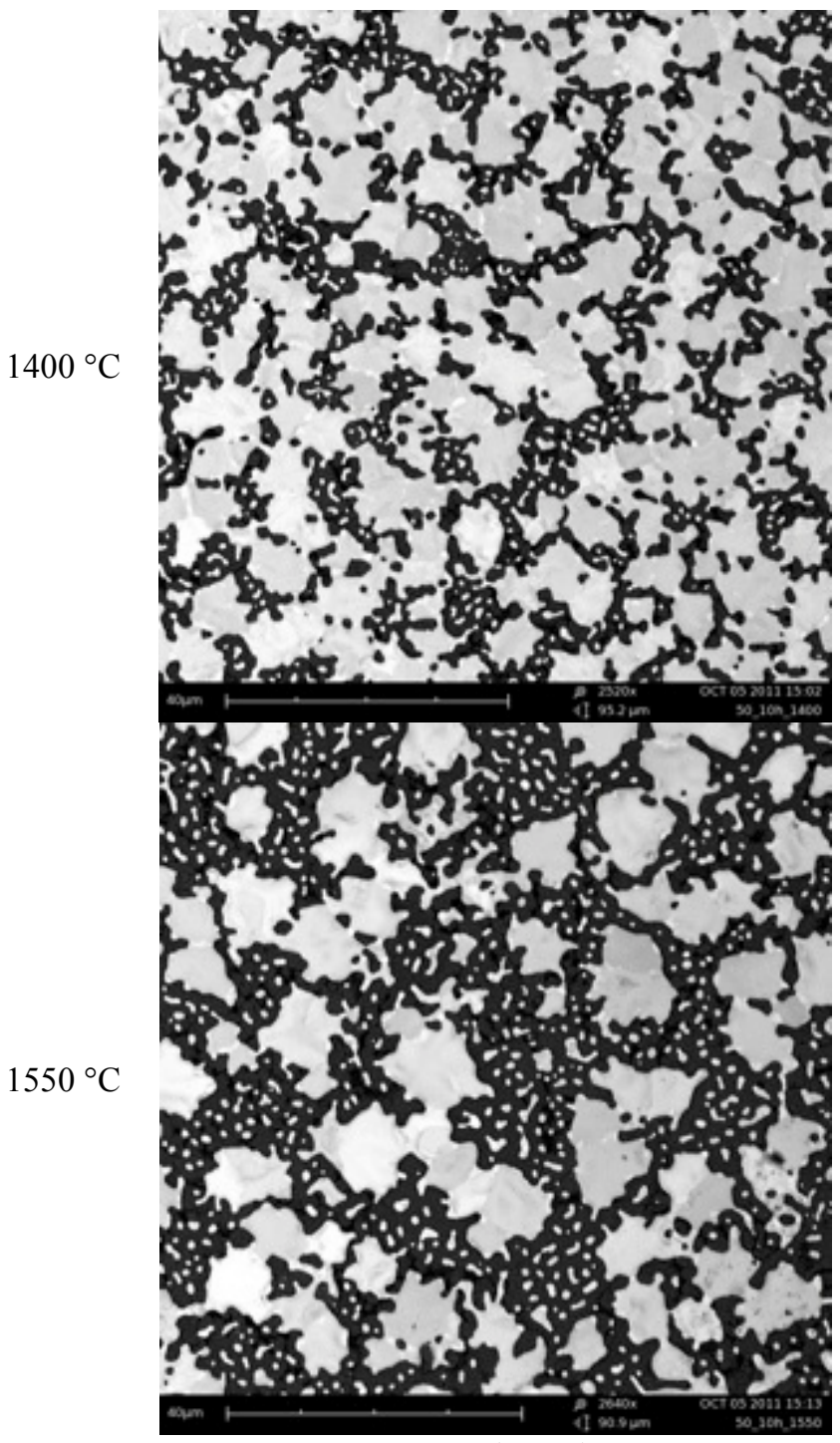

$\mathrm{H}_{2}-\mathrm{Ar}(10-90)$

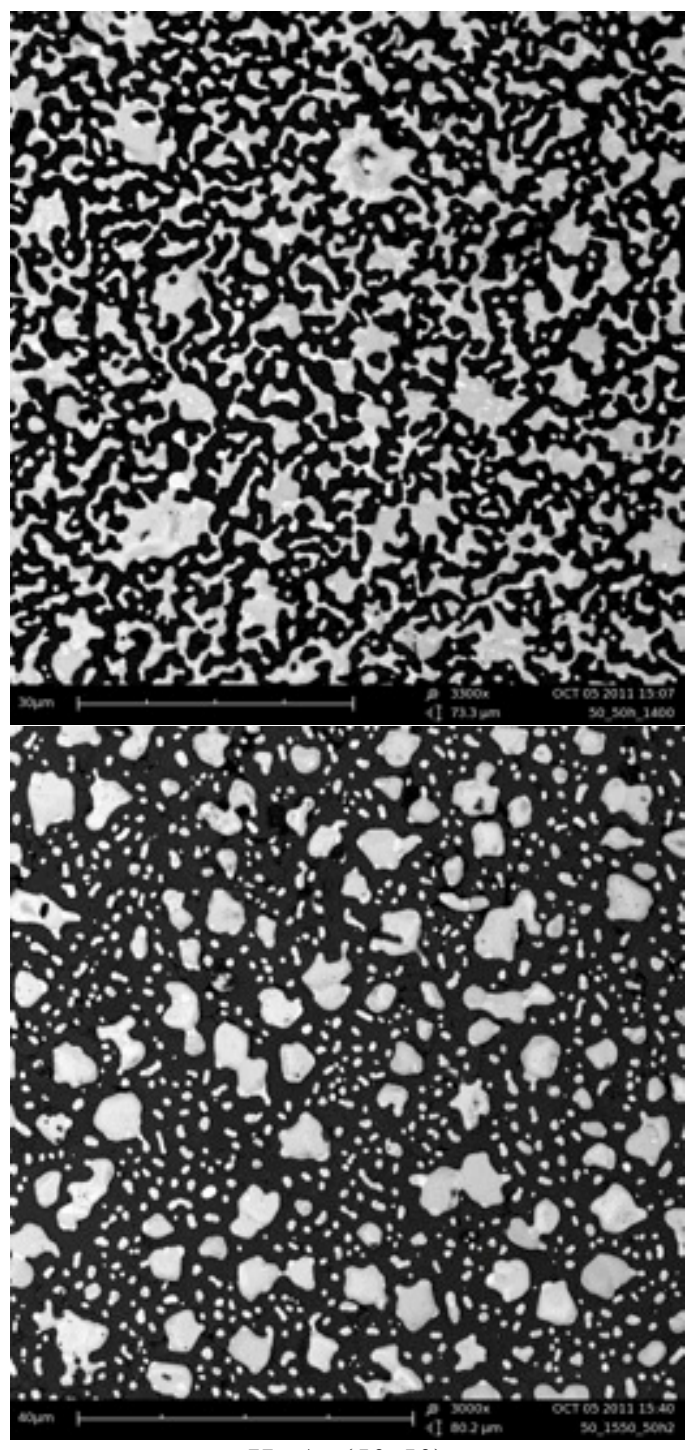

$\mathrm{H}_{2}-\operatorname{Ar}(50-50)$

Figure 8-18 $50 \mathrm{~nm}$ platinum thin film heated in the $\mathrm{H}_{2}$-Ar atmosphere for 1 hour

As is seen in all cases, the film is metastable and starts to make an array of islands. The

$200 \mathrm{~nm}$ thick samples are more stable, yet they also show the same results shown in figure 8-19. 
The island formation is not similar in each case. Increasing the temperature makes the island grow bigger but decreases the number of the islands. It could be said that increasing the temperature increases the densification of platinum. In the $200 \mathrm{~nm}$ samples, films are still stable and have not broken to the group of islands. However, the formations of the large grains are clear in figure 8-18. In addition to that, increasing the hydrogen concentration makes platinum islands distribute more evenly.

As discussed in section 2-4, at high temperature, thin films can break down to the array of islands. Srolovitz and Safran [90] showed the parameter $\beta=(R / a)^{2}\left(1+\gamma_{s} / \gamma_{v}\right)$ could determine the stability of a thin film. For $\beta$ values between $8-27$, the array of islands is in metastable equilibrium with respect to the continuous film. Increasing the $\beta$ more than 27 makes the island stable with respect to the film and for $\beta$ less than 8 , the arrays of islands will touch and reestablish the continuous film. The particle diameter (R) was calculated based on the $\beta$ formulation for the platinum/alumina system with data from the work of McLean and Hondros [160] to determine the stable-metastable-unstable boundary region, and is shown in table 8-2.

Table 8-2 Boundry condition of the stable-metasatble region for different film thickness

\begin{tabular}{lccc}
\hline & Stable $<$ & & $<$ Unstable \\
\hline $50 \mathrm{~nm}$ & 115 & \multirow{2}{*}{$R$} & 212 \\
$200 \mathrm{~nm}$ & 462 & & 850 \\
\hline
\end{tabular}

As indicted in table 8-2, the size of the islands are more than $200 \mathrm{~nm}$ in the $50 \mathrm{~nm}$ thick samples and more than 850 for $200 \mathrm{~nm}$ samples, yet the film is stable and does not fall apart. Increasing both the temperature and hydrogen concentration at the same time move atoms more freely and help to form islands, yet the film is still in the metastable state. It is possible that, one hour heating was not sufficient enough to achieve an equilibrium state. The microstructure of 
the $200 \mathrm{~nm}$ sample heated in $1400{ }^{\circ} \mathrm{C}$ for 8 hours in $\mathrm{H}_{2}-\mathrm{Ar}(10-90)$ atmosphere is shown in figure 8-18. It is clear that increasing the time leads to breaking in the film, but the platinum islands are still connected to each other. The islands do not have any particular shape or structure and are only formed due to high temperature. Upon quickly heating the sample to high temperature, platinum starts to react with alumina and changes the platinum-alumina surface energy. Because of that the samples shown in figure 8-18 are not in agreement with Srolovitz and Safran's criterion [90].

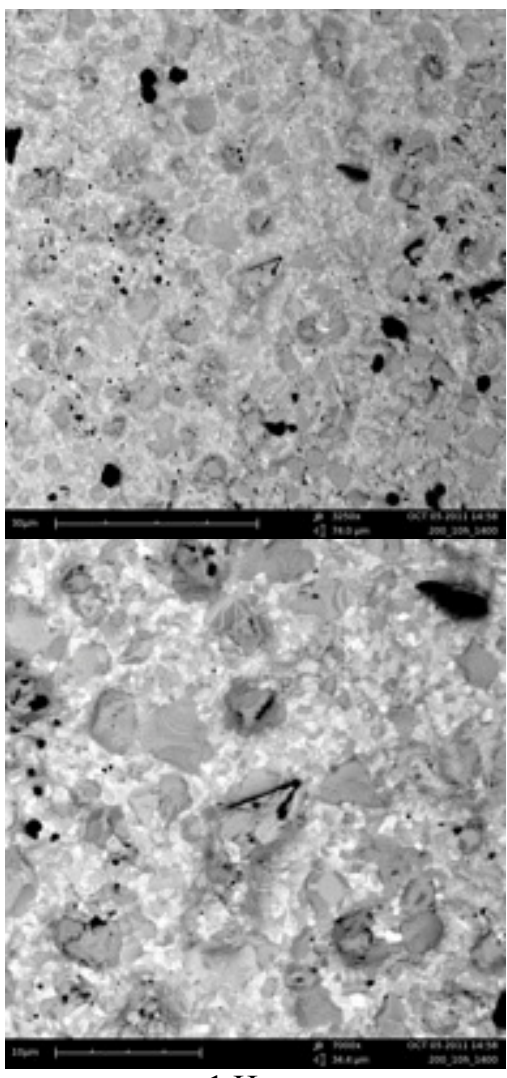

1 Hour

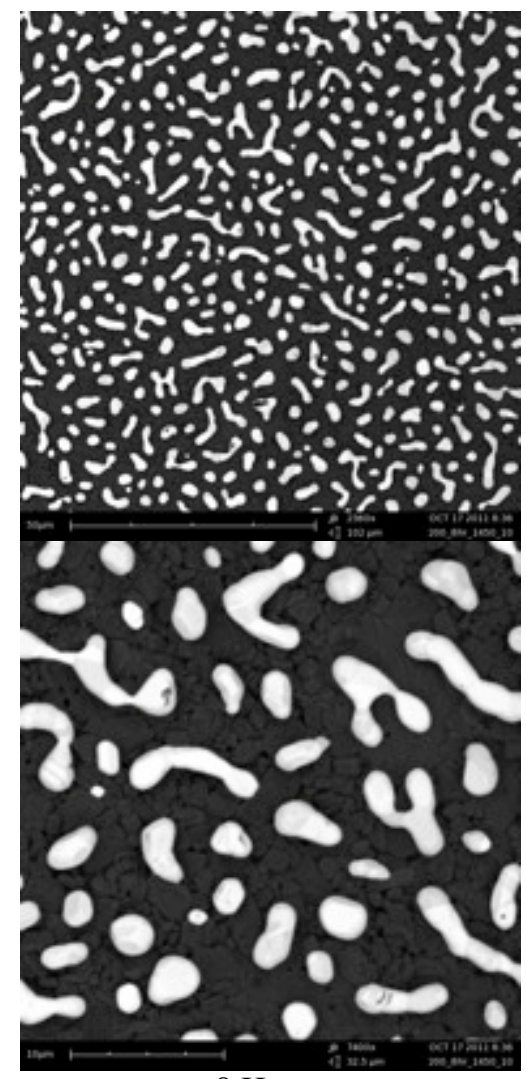

8 Hours

Figure 8-19 $200 \mathrm{~nm}$ platinum thin film rapidly heated in the $\mathrm{H}_{2}$-Ar (10-90) atmosphere at $1400{ }^{\circ} \mathrm{C}$

To verify the possibility of the proposed mechanism, the samples were heated slowly at the rate of $10^{\circ} \mathrm{C} / \mathrm{min}$. In this way, a platinum film has sufficient time to rupture. The samples 
then were kept for an hour and then were cool down at the same rate. The microstructures of the sample heated in $\mathrm{H}_{2}$ - $\mathrm{Ar}$ (10-90) atmosphere are shown in figure 8-20.

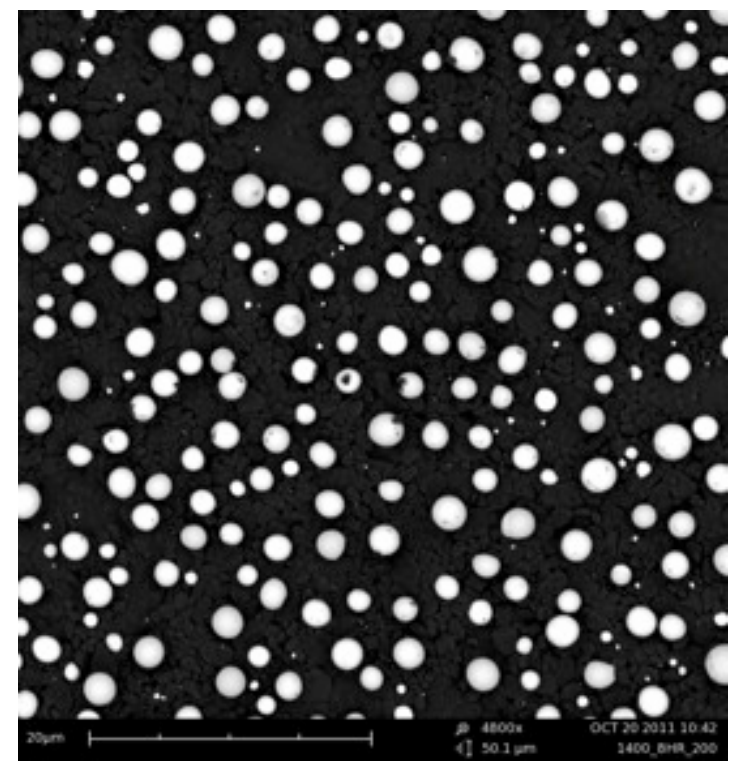

$1400{ }^{\circ} \mathrm{C}$

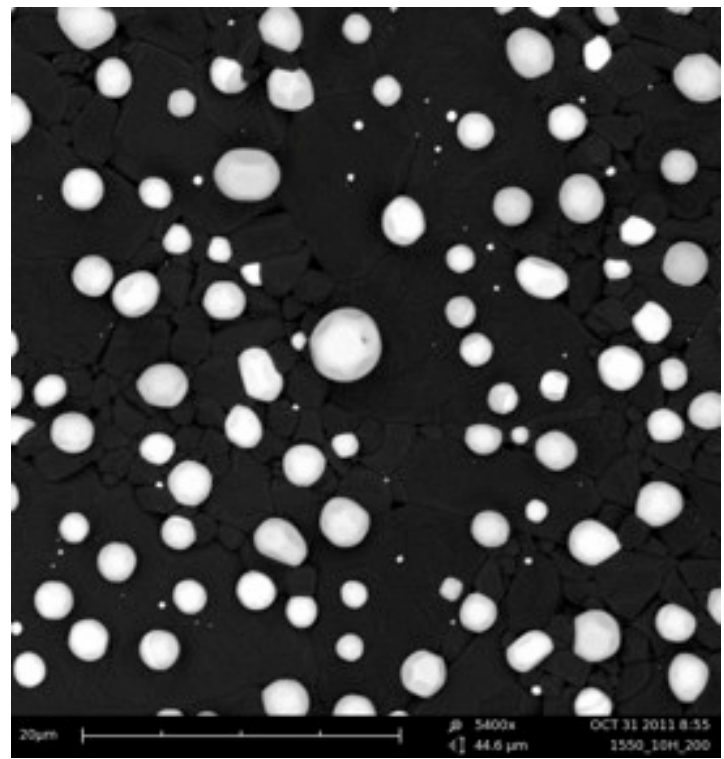

$1550{ }^{\circ} \mathrm{C}$

Figure 8-20 $200 \mathrm{~nm}$ platinum thin film heated in H2-Ar (10-90) atmosphere with $10^{\circ} \mathrm{C} / \mathrm{min}$ rate

As depicted in figure 8-20, platinum film breaks completely and forms particles. It is clear that the particles start to have a particular shape and are similar to the shape of the crystal observed in figure 7-10. In addition, the particle size distributions in two temperatures are completely different. The size distribution of the 50 and $200 \mathrm{~nm}$ particles heated to different temperatures and hydrogen concentrations were determined by Image J software and are shown in tables 8-3 and 8-4.

The size of the platinum particles increased by increasing the temperature in the constant hydrogen concentration. Increasing the temperature from $1450{ }^{\circ} \mathrm{C}$ to $1550^{\circ} \mathrm{C}$ increased the particle size about $60 \%$. However, increasing the hydrogen concentration did not follow the same trend. Figures 8-21 and 8-22 showed the microstructure of the 50 and $200 \mathrm{~nm}$ thick film heated at $1450^{\circ} \mathrm{C}$ in different hydrogen concentration. The $50 \mathrm{~nm}$ film breaks to almost spherical particles, while the $200 \mathrm{~nm}$ film creates spherical particles with some sharp edges. Particle size 
increases by increasing hydrogen concentration in the $50 \mathrm{~nm}$ platinum film and in contrast the number of particles in the area decreases with increasing the concentration. Increasing the hydrogen concentration enhances the migration of the small platinum particles and makes them fuse together. This mechanism could explain the behavior of the $50 \mathrm{~nm}$ films in different hydrogen concentrations.

Table 8-3 Particle size distribution of the $200 \mathrm{~nm}$ platinum thin film heated in different hydrogen concentration

\begin{tabular}{cccc}
\hline \multirow{2}{*}{$\begin{array}{c}\text { Hydrogen } \\
\text { Concentration }\end{array}$} & \multicolumn{3}{c}{ Temperature $\left({ }^{\circ} \mathrm{C}\right)$} \\
\cline { 2 - 4 } $10 \%$ & 1400 & 1450 & 1550 \\
\hline $20 \%$ & 2.40 & 2.40 & 3.83 \\
$50 \%$ & 1.37 & 1.46 & -- \\
\hline
\end{tabular}

Table 8-4 Particle size distribution of the $50 \mathrm{~nm}$ platinum thin film heated in different hydrogen concentration

\begin{tabular}{cccc}
\hline \multirow{2}{*}{$\begin{array}{c}\text { Hydrogen } \\
\text { Concentration }\end{array}$} & \multicolumn{3}{c}{ Temperature $\left({ }^{\circ} \mathrm{C}\right)$} \\
\cline { 2 - 4 } $10 \%$ & 1400 & 1450 & 1550 \\
\hline $20 \%$ & 0.27 & 0.29 & 0.47 \\
$50 \%$ & 0.25 & 0.40 & -- \\
\hline
\end{tabular}

Figure 8-23 showed the behavior of the particles' number density (number of particles/area) with variation of the particle size for the 50 and $200 \mathrm{~nm}$ films in different conditions. In the $50 \mathrm{~nm}$ films, while particle size increases from $0.25 \mu \mathrm{m}$ to $0.47 \mu \mathrm{m}$, the density of the particle decreases from 1.6 to 0.2 . These results are in agreement with the proposed mechanism for platinum particles growth.

The behavior of the $200 \mathrm{~nm}$ film is completely different. It is clear from the data in table 8-3 that the diameter of the particle first decreases and then increases. However, the average number of particles' density is almost 0.1 and does not change drastically with increasing the particle size. The $200 \mathrm{~nm}$ film breaks into the macron-sized particles. It could be said that the large size of particles prevents the their migration and because of that the number of particles is 
almost constant. As seen before in chapter 7, alumina can be absorbed into the platinum and expands it at high temperatures. This could explain the increasing of size with temperature, yet increasing the hydrogen concentration is changing this behavior. As discussed before, hydrogen increases the densification of the platinum and hence decreases the particles' diameter. In addition to that, in hydrogen concentration more than $20 \%$, alumina covers the surface of some platinum particles randomly and encapsulates some of the platinum crystals. Increasing the hydrogen concentration can expand the platinum by increasing the adsorption of alumina or by increasing the thickness of the covered layer.

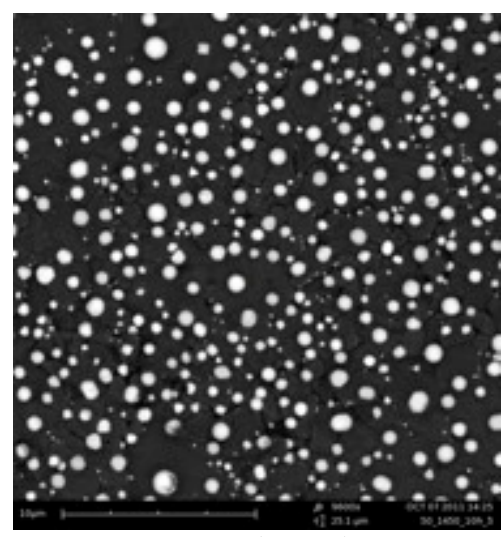

$\mathrm{H}_{2}-\mathrm{Ar}(10-90)$

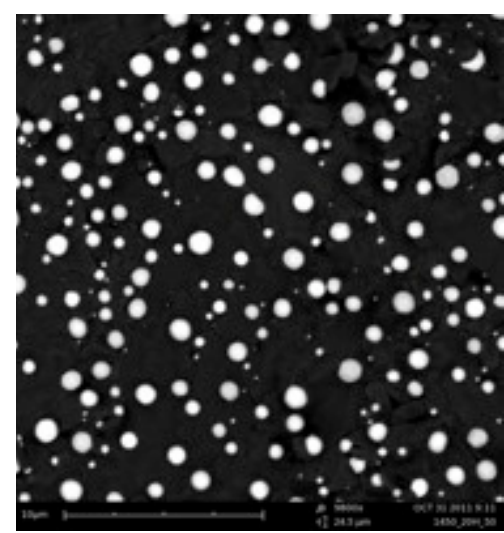

$\mathrm{H}_{2}-\mathrm{Ar}(20-80)$

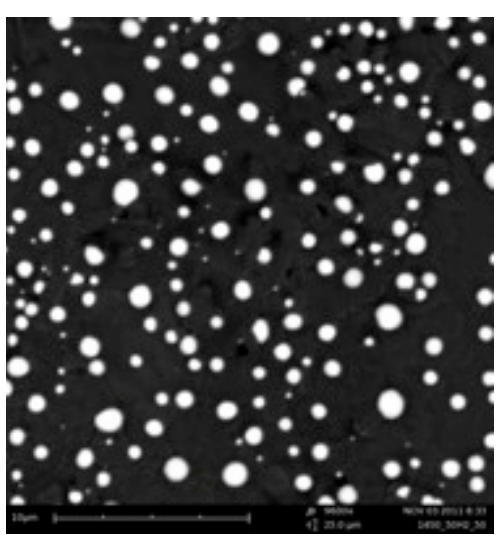

$\mathrm{H}_{2}-\mathrm{Ar}(50-50)$

Figure 8-21 Microstructure of the $50 \mathrm{~nm}$ thin film heated at $1450{ }^{\circ} \mathrm{C}$ in different hydrogen concentration

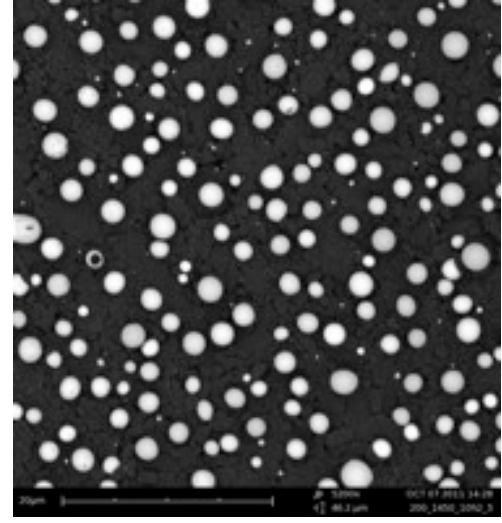

$\mathrm{H}_{2}-\mathrm{Ar}(10-90)$

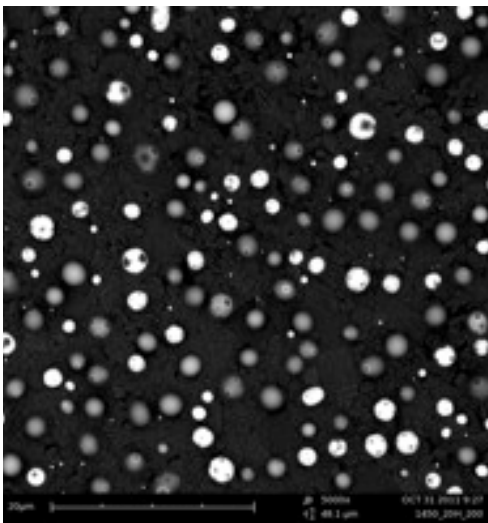

$\mathrm{H}_{2}-\mathrm{Ar}(20-80)$

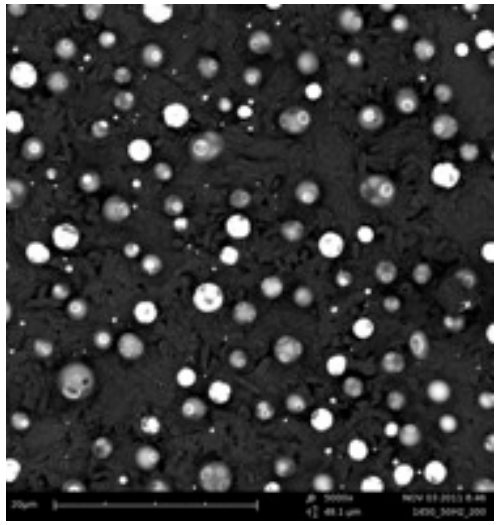

$\mathrm{H}_{2}-\operatorname{Ar}(50-50)$

Figure 8-22 Microstructure of the $200 \mathrm{~nm}$ thin film heated at $1450^{\circ} \mathrm{C}$ in different hydrogen concentration

Figure 8-23 indicates that the densification behavior in platinum thin films is similar to the sintering path of the alumina particles shown in figure 2-5 and followed the same pattern. 


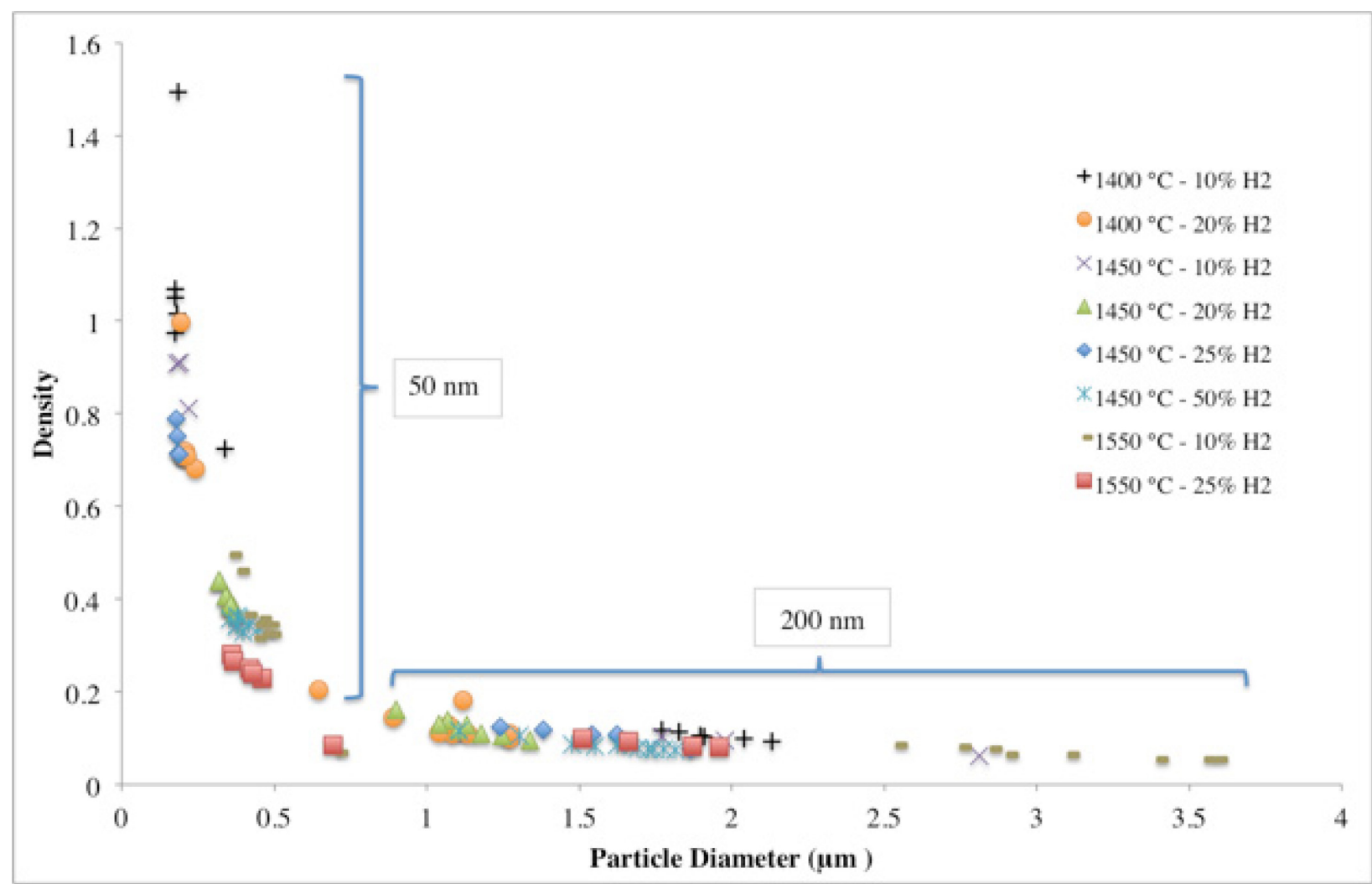

Figure 8-23 Variation of the number density of platinum particles with the particle size for 50 and $200 \mathrm{~nm}$ platinum thin films heated in different temperatures and hydrogen concentration

The distribution of the encapsulated particles is completely random, as shown in figure 822. The particles formed in the $200 \mathrm{~nm}$ film are almost spherical in nature with facet in (100) and (111) faces. Surface free energy of the (100) and (111) faces are less than other areas, and because of that, when alumina starts to cover the platinum particles, flat surfaces are not covered completely. It is clear that the type of encapsulation that happened in figure 8-22 is different from the one observed in figures 8-16 and 8-17. As mentioned before, alumina does not cover the particles with a diameter less than $10 \mu \mathrm{m}$, however here the decoration is almost independent of the size. It seems that atomic migration is the dominant mechanism of alumina migration. High surface area of the platinum thin film increases the interaction force between platinum and alumina and because of that, the atomic migration is the most probable mechanism, as stated by Baker et al. [81, 82]. The encapsulation mechanism of palladium particles with $\mathrm{TiO} 2$ [26] could 
explain the alumina migration in this case. Hydrogen reduces the alumina and creates a high concentration aluminum cations layer. The contact between the oxide and the metal will establish positive space charges in alumina. They drive the outward diffusion of $\mathrm{Al}^{3+}$ but hinder the outward diffusion of oxygen anions $\left(\mathrm{O}^{2-}\right)$. On the other hand, the outward diffusion of $\mathrm{Al}^{3+}$ will gradually weaken the positive space charges, which allows the outward diffusion of oxygen anions, thus favoring the formation of alumina on the surface. Due to the difference of the surface energy of the oxide and the metal, alumina tends to cover the surface of the metal crystals, resulting in encapsulation.
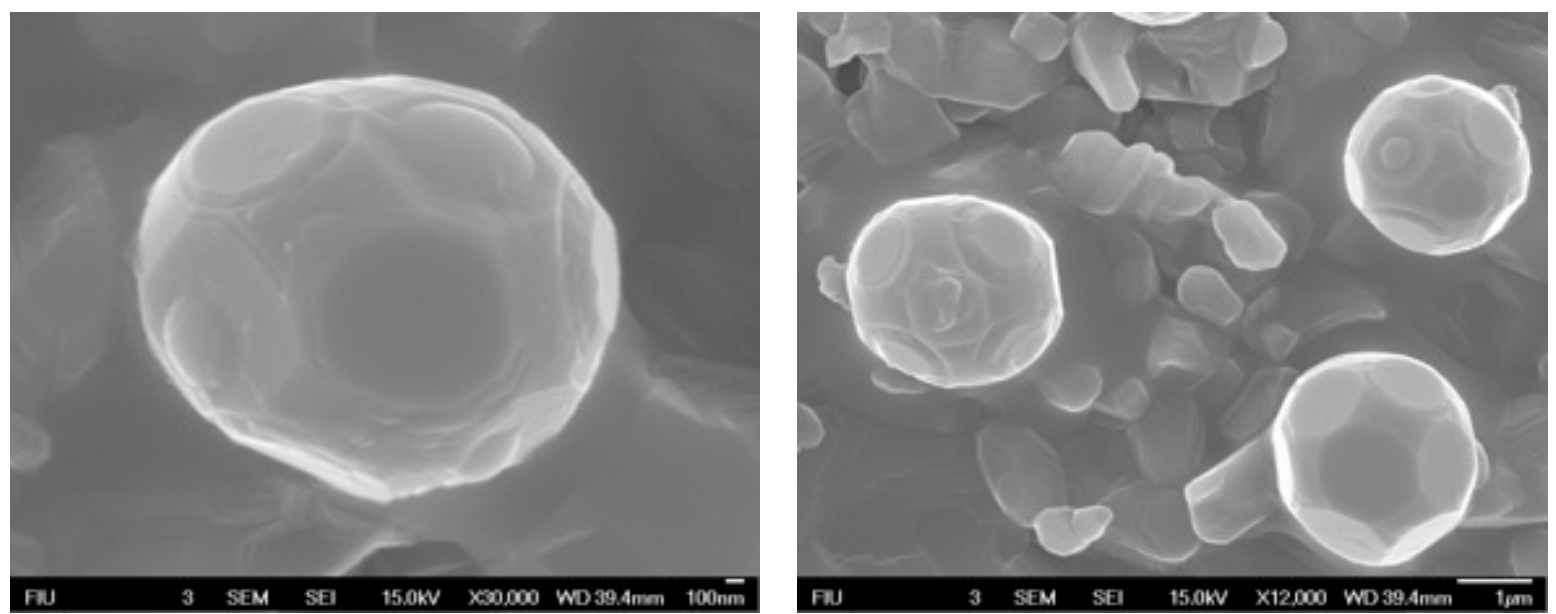

Figure 8-24 High magnification of SEM image of platinum crystal from $200 \mathrm{~nm}$ film heated at 1450 in the $\mathrm{H2}-\mathrm{Ar}$ (20-80) atmosphere

High magnification image of particles form in $\mathrm{H}_{2}-\mathrm{Ar}(20-80)$ atmosphere at $1450{ }^{\circ} \mathrm{C}$ is shown in figure 8-24. Lee et al.[151] showed that upon annealing in $1200^{\circ} \mathrm{C}$, platinum particles start to rotate. They suggest that it could be due to crystallographic axis alignment of a particle with the oxide support, however the exact nature of rotation force is unknown. Figure 8-24 shows different platinum crystals have different orientation, which could be due to the different crystal orientation of polycrystalline aluminum oxide. 


\section{Conclusion}

- Increasing the platinum particle size decreases the particles densification. In addition to that large platinum particles have a lower surface area, which decreases the reaction of platinum with oxygen at high temperatures. Overally, larger platinum particles are better candidates in cofired ink for multilayer ceramic technology.

- The optimum heating-firing profile consists of the rate of $1-2^{\circ} \mathrm{C} / \mathrm{min}$ up to $450{ }^{\circ} \mathrm{C}$ for binder to burn-out and the rate of $5-10^{\circ} \mathrm{C} / \mathrm{min}$ up to $1550^{\circ} \mathrm{C}$ for sintering of the materials.

- In general, non-reducing like air, argon or vacuum are not an optimum environment to develop a high-density via structure.

- In reduced atmospheres, the firing temperature could be adjusted by the size of platinum particle. Larger platinum particles could be fired in temperatures lower than $1550{ }^{\circ} \mathrm{C}$.

- In reduced atmosphere, at high temperatures, platinum absorbs alumina particles from the sidewalls of the via. At temperature above $1500^{\circ} \mathrm{C}$, these absorbed particles could be melted completely and be mixed with platinum to create a very dense structure.

- Tungsten could be used as the best metallization for a composite structure with platinum in an ink formulation. The platinum/ tungsten ratio is a major factor to control the shrinkage of the feedthrough assembly. A graded composite sample with some internal tungsten layer and increasing platinum/tungsten ratio in the outer layers with pure platinum in the top layers showed promising results.

- Platinum starts to react with alumina at temperatures above $1350{ }^{\circ} \mathrm{C}$, however the recrystallization and the change of alumina structure can only be achieved at temperatures above $1550^{\circ} \mathrm{C}$. 
- Upon heating the platinum film coated on an alumina substrate in a reduced atmosphere, the film contracts and creates a series of platinum particles. Alumina could cover the surface of these particles based on heating temperature and environment. Different mechanisms are proposed to describe this behavior of alumina.

- Platinum particles created in a reduced atmosphere could rotate to be in alignment with the crystallographic axis of the oxide support. In addition to that, the shape of particles could change with the concentration of hydrogen. Increasing the hydrogen concentration changes the particle shape from the truncated octahedron to spherical with faceted faces on some edges to an almost spherical particle. 


\section{Recommendation for future research}

- Two hydrogen furnaces used in this project, exhibited different densification behavior. The study of the effect of different furnaces and heating mechanisms on the densification and interaction of the platinum and alumina could be useful in developing the feedthrough structure.

- The absorbed alumina into platinum, at high temperature in a reduced atmosphere, creates a very dense structure. This structure is completely unified as observed with backscatter image. The study of the interface area of the platinum/alumina with TEM in this area would be very helpful to understand this behavior.

- Platinum particles could rotate to be in alignment with the crystallographic axis of oxide support due to the polycrystalline nature of the alumina substrate. The study of the effect of oxide crystallographic orientation on the alignment of the platinum particles with TEM could be useful to understand the platinum/alumina bonding mechanism. 


\section{References}

1. Tauster SJ, Fung SC, Baker RTK, Horsley JA. Strong interactions in supported-metal catalysts. Science. 1981;211(4487):1121.

2. $\quad \mathrm{Fu} \mathrm{Q}$, Wagner T. Interaction of nanostructured metal overlayers with oxide surfaces. Surface Science Reports. 2007;62(11):431-98.

3. Merrill DR, Bikson M, Jefferys JGR. Electrical stimulation of excitable tissue: design of efficacious and safe protocols. Journal of Neuroscience Methods. 2005;141(2):171-98.

4. Panfilov P, Bochegov A, Yermakov A. The Transition Layer in Platinum-Alumina. Platinum Metals Review. 2004;48(2):47-55.

5. Allen RV, Borbidge WE. Solid state metal-ceramic bonding of platinum to alumina. Journal of Materials Science 1983;18:2835-43.

6. Suppel KP, Forrester JS, Suaning GJ, Kisi EH. A Study of the Platinum/Alumina Interface. Advances in Science and Technology. 2006;45:1417-22.

7. Ruckenstein E, $\mathrm{Hu}$ XD. Mechanism of redispersion of supported metal catalysts in oxidative atmospheres. Langmuir. 1985;1(6):756-60.

8. Andersson S, Brühwiler PA, Sandell A, Frank M, Libuda J, Giertz A, et al. Metal-oxide interaction for metal clusters on a metal-supported thin alumina film. Surface Science. 1999;442(1):L964-L70.

9. Linsmeier C, Taglauer E. Strong metal-support interactions on rhodium model catalysts. Applied Catalysis A: General. 2010;Article in Press.

10. Chu YF, Ruckenstein E. On the sintering of platinum on alumina model catalyst. Journal of Catalysis. 1978;55(3):281-98.

11. Mulder CAM, Klomp JT. On The Internal Structure of $\mathrm{Cu}$ - and Pt- Sapphire Interfaces. J Phys Colloques. [10.1051/jphyscol:1985412]. 1985;46(C4):C4-111-C4-6.

12. Hwang CP, Yeh CT. Platinum-oxide species formed by oxidation of platinum crystallites supported on alumina. Journal of Molecular Catalysis A: Chemical. 1996;112(2):295-302.

13. Bronger W, Wrzesien K, Müller P. High temperature phase transitions in Al1+xPt3-x. Solid State Ionics. 1997;101-103(Part 1):633-40. 
14. Penner S, Wang D, Su DS, Rupprechter G, Podloucky R, Schlögl R, et al. Platinum nanocrystals supported by silica, alumina and ceria: metal-support interaction due to hightemperature reduction in hydrogen. Surface Science. 2003;532-535:276-80.

15. Zhong X, Zhu J, Liu J. Study of the interfacial structure of a Pt/ $\alpha-\mathrm{Al} 2 \mathrm{O} 3$ model catalyst under high-temperature hydrogen reduction. Journal of Catalysis. 2005;236(1):9-13.

16. Luo M-F, Ten M-H, Wang C-C, Lin W-R, Ho C-Y, Chang B-W, et al. TemperatureDependent Oxidation of Pt Nanoclusters on a Thin Film of Al2O3 on NiAl(100). The Journal of Physical Chemistry C. 2009;113(28):12419-26.

17. Sinnott SB, Dickey EC. Ceramic/metal interface structures and their relationship to atomic- and meso-scale properties. Materials Science and Engineering: R: Reports. 2003;43(12):1-59.

18. Scheu C, Gao M, Oh S, Dehm G, Klein S, Tomsia A, et al. Bonding at copper-alumina interfaces established by different surface treatments: a critical review. Journal of Materials Science. 2006;41(16):5161-8.

19. Nakagawa N, Hwang HY, Muller DA. Why some interfaces cannot be sharp. Nature Materials. 2006;5(3):204-9.

20. Moya JS, Lopez-Esteban SPC. The challenge of ceramic/metal microcomposites and nanocomposites. Progress in Materials Science. 2007;52(7):1017-90.

21. Schierbaum KD, Fischer S, Torquemada MC, de Segovia JL, Román E, Martin-Gago JA. The interaction of Pt with TiO2(110) surfaces: a comparative XPS, UPS, ISS, and ESD study. Surface Science. 1996;345(3):261-73.

22. Linsmeier $\mathrm{C}, \mathrm{Kn} \sqrt{ }$ ozinger H, Taglauer E. Depth profile analysis of strong metal-support interactions on model catalysts. Ion Beam Analysis. 1996;118(1-4):533-40.

23. Ertl G, Knözinger H, Weitkamp J. Handbook of Heterogeneous Catalysis. Germany: VCH: Weinheim; 1997.

24. Haruta M. Size- and support-dependency in the catalysis of gold. Catalysis Today. 1997;36(1):153-66.

25. Henry CR. Surface studies of supported model catalysts. Surface Science Reports. 1998;31(7,Äì):231-325.

26. Fu Q, Wagner T, Olliges S, Carstanjen HD. Metal-Oxide Interfacial Reactions: Encapsulation of Pd on TiO2 (110). The Journal of Physical Chemistry B. 2005;109(2):944-51. 
27. Flynn PC, Wanke SE. A model of supported metal catalyst sintering : I. Development of model. Journal of Catalysis. 1974;34(3):390-9.

28. Flynn PC, Wanke SE. A model of supported metal catalyst sintering: II. Application of model. Journal of Catalysis. 1974;34(3):400-10.

29. Wynblatt P, Gjostein NA. Supported metal crystallites. Progress in Solid State Chemistry. 1975;9(Journal Article):21-58.

30. Fiedorow RMJ, Wanke SE. The sintering of supported metal catalysts: I. Redispersion of supported platinum in oxygen. Journal of Catalysis. 1976;43(1-3):34-42.

31. Fiedorow RMJ, Chahar BS, Wanke SE. The sintering of supported metal catalysts : II. Comparison of sintering rates of supported $\mathrm{Pt}$, Ir, and Rh catalysts in hydrogen and oxygen. Journal of Catalysis. 1978;51(2):193-202.

32. Ruckenstein E, Chu YF. Redispersion of platinum crystallites supported on alumina: Role of wetting. Journal of Catalysis. 1979;59(1):109-22.

33. Harris PJF. The sintering of platinum particles in an alumina-supported catalyst: Further transmission electron microscopy studies. Journal of Catalysis. 1986;97(2):527-42.

34. Sushumna I, Ruckenstein E. Events Observed and Evidence for Crystallite in Pt / A1203 Catalysts. Journal of Catalysis. 1988;109:433-62.

35. Vaarkamp M, Miller JT, Modica FS, Koningsberger DC. On the Relation between Particle Morphology, Structure of the Metal-Support Interface, and Catalytic Properties of $\mathrm{Pt} / \mathrm{E} \geq-\mathrm{Al2O}$. Journal of Catalysis. 1996;163(2):294-305.

36. Tauster SJ, Fung SC, Garten RL. Strong metal-support interactions. Group 8 noble metals supported on titanium dioxide. Journal of the American Chemical Society. 1978;100(1):170-5.

37. Tauster SJ. Strong metal-support interactions. Accounts of Chemical Research. 1987;20(11):389-94.

38. Domenichini B, Flank AM, Lagarde P, Bourgeois S. Interfacial reaction between deposited molybdenum and TiO2 $(1 \& \# \times a 0 ; 1 \& \# x a 0 ; 0)$ surface: role of the substrate bulk stoichiometry. Surface Science. 2004;560(1, Äi3):63-78.

39. Marien J, Wagner T, Duscher G, Koch A, R ${ }^{\circ}{ }^{\circ}$ le M. Nb on (110) TiO2 (rutile): growth, structure, and chemical composition of the interface. Surface Science. 2000;446(3):219-28.

40. Wagner T, Fu Q, Winde C, Tsukimoto S, Phillipp F. A Comparative Study of the Growth of $\mathrm{Cr}$ on (110)TiO2; Rutile, (0001) $\alpha$-A2O3; and (100)SrTiO3; Surfaces. Interface Science. 2004;12(1):117-26. 
41. Pretorius R, Harris JM, Nicolet MA. Reaction of thin metal films with $\mathrm{SiO} 2$ substrates. Solid-State Electronics. 1978;21(4):667-75.

42. Zhao XA, Kolawa E, Nicolet MA. Reaction of thin metal films with crystalline and amorphous Al[sub 2] O[sub 3]. Journal of Vacuum Science \& Technology A: Vacuum, Surfaces, and Films. 1986;4(6):3139-41.

43. Lamber R, Jaeger N. On the reaction of Pt with $\mathrm{SiO} 2$ substrates: Observation of the Pt3Si phase with the Cu3Au superstructure. Journal of Applied Physics. 1991;70:457-.

44. Wang D, Penner S, Su DS, Rupprechter G, Hayek K, Schl $\sqrt{\partial g l}$ R. Silicide formation on a $\mathrm{Pt} / \mathrm{SiO} 2$ model catalyst studied by TEM, EELS, and EDXS. Journal of Catalysis. 2003;219(2):434-41.

45. Min BK, Santra AK, Goodman DW. Understanding silica-supported metal catalysts: $\mathrm{Pd} /$ silica as a case study. Catalysis Today. 2003;85(2,Äi4):113-24.

46. Bernal S, Calvino JJ, Cauqui MA, Gatica JM, L $\sqrt{ } \geq$ pez Cartes C, P $\sqrt{ }$ Crez Omil JA, et al. Some contributions of electron microscopy to the characterisation of the strong metal, Äisupport interaction effect. Fundamentals of Catalysis and Applications to Environmental Problems. 2003;77(4):385-406.

47. Zhong Q, Ohuchi FS. Surface science studies on the Ni/Al[sub 2]O[sub 3] interface. Journal of Vacuum Science \& Technology A: Vacuum, Surfaces, and Films. 1990;8(3):2107-12.

48. Raj R, Saha A, An L, Hasselman DPH, Ernst P. Ion exchange at a metal,Äìceramic interface. Acta Materialia. 2002;50(5):1165-76.

49. $\mathrm{Yu}$ Y, Mark J, Ernst F, Wagner T, Raj R. Diffusion reactions at Al-MgAl2O4 interfaces - and the effect of applied electric fields. Journal of Materials Science. 2006;41(23):7785-97.

50. Schulz H, Ritapal K, Bronger W, Klemm W. Über die Reaktion von Elementen der achten Nebengruppe mit Oxiden unedler Metalle im Wasserstoffstrom (About the reaction of elements of the eighth group with oxides of base metals in a hydrogen stream). Zeitschrift für anorganische und allgemeine Chemie. 1968;357:299-313.

51. Ruckenstein E, Malhotra ML. Splitting of platinum crystallites supported on thin, nonporous alumina films. Journal of Catalysis. 1976;41(2):303-11.

52. Sushumna I, Ruckenstein E. Redispersion of Pt/alumina via film formation. Journal of Catalysis. 1987;108(1):77-96.

53. den Otter GJ, Dautzenberg FM. Metal-support interaction in Pt/A12O3 catalysts. Journal of Catalysis. 1978;53(1):116-25. 
54. Kunimori K, Ikeda Y, Soma M, Uchijima T. Metal-support interaction in aluminasupported Pt catalysts. Journal of Catalysis. 1983;79(1):185-95.

55. Klomp J. Ceramic-metal reactions and their effect on the interface microstructure. Ceramic microstructures '86: Role of interfaces; Proceedings of the International Materials Symposium; 28-31 July 1986; Berkeley, CA; UNITED STATES1987. p. 307-17.

56. Conner WC, Falconer JL. Spillover in Heterogeneous Catalysis. Chemical Reviews. 1995 1995/05/01;95(3):759-88.

57. Candau JFC, Conner WC. The spillover of hydrogen onto silica: IV. The use of scanning FTIR to follow spillover from a point source. Journal of Catalysis. 1987;106(2):378-85.

58. Becher PF, Murday JS. Thick film adherence fracture energy: influence of alumina substrates. Journal of Materials Science. 1977;12(6):1088-94.

59. Ivanova AS, Slavinskaya EM, Gulyaev RV, Zaikovskii VI, Stonkus ûê, Danilova IG, et al. Metal-support interactions in $\mathrm{Pt} / \mathrm{Al} 2 \mathrm{O} 3$ and $\mathrm{Pd} / \mathrm{Al} 2 \mathrm{O} 3$ catalysts for $\mathrm{CO}$ oxidation. Applied Catalysis B: Environmental. 2010;97(1-2):57-71.

60. De Graef M, Dalgleish BJ, Turner MR, Evans AG. Interfaces between alumina and platinum: Structure, bonding and fracture resistance. Acta Metallurgica et Materialia. 1992;40(Supplement 1):S333-S44.

61. Lu H, Svehla MJ, Skalsky M, Kong C, Sorrell CC. Pt-A12O3 interfacial bonding in implantable hermetic feedthroughs: Morphology and orientation. Journal of Biomedical Materials Research Part B: Applied Biomaterials. 2011:n/a-n/a.

62. Santala MK, Radmilovic V, Giulian R, Ridgway MC, Gronsky R, Glaeser AM. The orientation and morphology of platinum precipitates in sapphire. Acta Materialia. 2011;59(12):4761-74.

63. Cole SS, Sommer G. Glass-Migration Mechanism of Ceramic-to-Metal Seal Adherence. Journal of the American Ceramic Society. [10.1111/j.1151-2916.1961.tb15376.x]. $1961 ; 44(6): 265-71$.

64. Cole SS, Jr , Hynes FJ. Some Parameters Affecting Ceramic-to-Metal Seal Strength of a High-alumina Body The Bulletin of the American Ceramic Society. 1958;37:135 - 8.

65. Floyd JR. Effect of composition and crystal size of alumina ceramics on metal to ceramics bond strength. The Bulletin of the American Ceramic Society. 1963;42(2):66 - 70.

66. Twentyman ME. High-temperature metallizing, Part 1. The mechanism of glass migration in the production of metal-ceramic seals. Journal of Materials Science. 1975;10(5):765-76. 
67. Behrens G, Heuer AH. Microstructural Characterization of Cofired Tungsten-Metallized High-Alumina Electronic Substrates. Journal of the American Ceramic Society. [10.1111/j.11512916.1992.tb05510.x]. 1992;75(10):2815-24.

68. Arthur ME, Fussell LE. Effect of sintering condition on Al2O3-Mo-Mn bond strengths. The Bulletin of the American Ceramic Society. 1971;50(12):982-4.

69. Selman GL, Spender MR, Darling AS. The Wetting of Platinum and Its Alloys By Glass I, Contact Angle Determination Between Glass and Pure Platinum. Platinum Metals Review. 1965;9(3):92-9.

70. Gland JL, Sexton BA, Fisher GB. Oxygen interactions with the Pt(111) surface. Surface Science. 1980;95(2-3):587-602.

71. Cabrera N, Mott NF. Theory of the oxidation of metals. Reports on Progress in Physics. 1949;12(1):163.

72. $\mathrm{Fu} \mathrm{Q}$, Wagner T. On the tunability of chemical reactions at metal,Äìoxide interfaces. Surface Science. 2005;574(2-3):L29-L34.

73. Fu Q, Wagner T. Metal/Oxide Interfacial Reactions:Oxidation of Metals on SrTiO3 (100) and TiO2 (110). The Journal of Physical Chemistry B. 2005;109(23):11697-705.

74. Kingston RH, Neustadter SF. Calculation of the Space Charge, Electric Field, and Free Carrier Concentration at the Surface of a Semiconductor. Journal of Applied Physics. 1955;26(6):718-20.

75. Meschel SV, Kleppa OJ. Standard enthalpies of formation of $5 \mathrm{~d}$ aluminides by hightemperature direct synthesis calorimetry. Journal of Alloys and Compounds. 1993;197(1):75-81.

76. Kim DE, Manga VR, Prins SN, Liu ZK. First-principles calculations and thermodynamic modeling of the Al,ÄiPt binary system. Calphad. 2011;35(11):20-9.

77. Huizinga T, van 'T Blik HFJ, Vis JC, Prins R. XPS investigations of Pt and Rh supported on $\alpha-\mathrm{Al} 2 \mathrm{O} 3$ and TiO2. Surface Science. 1983;135(1-3):580-96.

78. Gao Y, Liang Y, Chambers SA. Thermal stability and the role of oxygen vacancy defects in strong metal support interaction, $\mathrm{Pt}$ on $\mathrm{Nb}$-doped TiO2(100). Surface Science. 1996;365(3):638-48.

79. Ruckenstein E, Pulvermacher B. Growth kinetics and the size distributions of supported metal crystallites. Journal of Catalysis. 1973;29(2):224-45. 
80. Simonsen SrB, Chorkendorff I, Dahl Sr, Skoglundh M, Sehested J, Helveg S. Ostwald ripening in a $\mathrm{Pt} / \mathrm{SiO} 2$ model catalyst studied by in situ TEM. Journal of Catalysis.

2011;281(1):147-55.

81. Baker RTK, Prestridge EB, Garten RL. Electron microscopy of supported metal particles : I. Behavior of Pt on titanium oxide, aluminum oxide, silicon oxide, and carbon. Journal of Catalysis. 1979;56(3):390-406.

82. Baker RTK, Prestridge EB, Garten RL. Electron microscopy of supported metal particles II. Further studies of Pt/TiO2. Journal of Catalysis. 1979;59(2):293-302.

83. Straguzzi GI, Aduriz HR, Gigola CE. Redispersion of platinum on alumina support. Journal of Catalysis. 1980;66(1):171-83.

84. Arai M, Ishikawa T, Nakayama T, Nishiyama Y. Effects of metal--support interaction and temperature on the sintering of Pt and Ag particles supported on inorganic solids. Journal of colloid and interface science. 1984;97(1):254-65.

85. Yao HC, Sieg M, Plummer JHK. Surface interactions in the Pt/ $\gamma$-A12O3 system. Journal of Catalysis. 1979;59(3):365-74.

86. Wang $\mathrm{T}$, Schmidt LD. Intraparticle redispersion of $\mathrm{Rh}$ and $\mathrm{Pt}-\mathrm{Rh}$ particles on $\mathrm{SiO} 2$ and A12O3 by oxidation-reduction cycling. Journal of Catalysis. 1981;70(1):187-97.

87. Stulga JE, Wynblatt P, Tien JK. Particle splitting and redispersion phenomena in model alumina-supported platinum catalysts. Journal of Catalysis. 1980;62(1):59-69.

88. Mullins WW. Theory of Thermal Grooving. Journal of Applied Physics. 1957;28(3):3339.

89. Srolovitz DJ, Safran SA. Capillary instabilities in thin films. II. Kinetics. Journal of Applied Physics. 1986;60(1):255-60.

90. Srolovitz DJ, Safran SA. Capillary instabilities in thin films. I. Energetics. Journal of Applied Physics. 1986;60(1):247-54.

91. Jiran E, Thompson C. Capillary instabilities in thin films. Journal of Electronic Materials. 1990;19(11):1153-60.

92. Rha JJ, Park JK. Stability of the grain configurations of thin films---A model for agglomeration. Journal of Applied Physics. 1997;82(4):1608-16.

93. Galinski H, Ryll T, Elser P, Rupp JLM, Bieberle-Hütter, A., Gauckler LJ. Agglomeration of Pt thin films on dielectric substrates. Physical Review B. 2010;82(23):235415. 
94. Ruckenstein E. Role of wetting in sintering and redispersion of supported metal crystallites. Journal of Crystal Growth. 1979;47(5-6):666-70.

95. Berg JC. Wettability: CRC; 1993.

96. Howatt GN, Breckenridge RG, Brownlow JM. Fabrication of Thin Ceramic Sheets for Capacitors. Journal of the American Ceramic Society. 1947;30(8):237-42.

97. Barlow FD, Elshabini A. Ceramic interconnect technology handbook: CRC; 2007.

98. Holzman E, Teti R, Dufour B, Miller S, editors. An hermetic coplanar waveguide-to-HDI microstrip microwave feedthrough. Microwave Symposium Digest, 1998 IEEE MTT-S International; 1998 7-12 Jun 1998.

99. Low YL, Scotti RE, Ramsey DA, Bolle CA, O'Neill SP, Nguyen KC. Packaging of Optical MEMS Devices. Journal of Electronic Packaging. 2003;125(3):325-8.

100. Bechtold F, editor. A comprehensive overview on today's ceramic substrate technologies. Microelectronics and Packaging Conference, 2009 EMPC 2009 European; 2009 15-18 June 2009.

101. Eustice A, Horowitz S, Travis A, Nishii N. Low temperature co-fireable ceramics for multilayer interconnect hybrids. IMC Proceedings, Kobe, Japan, May. 1986:28-30.

102. Nishigaki S, Fukuta J, Yano S, Kawabe H, Noda K, Fukaya M, et al., editors. A New Low Temperature Fireable Ag Multilayer Ceramic Substrate having Post-Fired Cu Conductor (LFC-2)1986.

103. Gongora-Rubio MR, Espinoza-Vallejos P, Sola-Laguna L, Santiago-Avil $\sqrt{ }$ Cs JJ. Overview of low temperature co-fired ceramics tape technology for meso-system technology (MsST). Sensors and Actuators A: Physical. 2001;89(3):222-41.

104. Imanaka Y. Multilayered Low Temperature Cofired Ceramics (LTCC) Technology: Springer US; 2005.

105. Golonka L. Technology and applications of low temperature cofired ceramic (LTCC) based sensors and microsystems. TECHNICAL SCIENCES. 2006;54(2).

106. Gitzen WH. Alumina as a ceramic material: ed. and comp.)1970.

107. Kingery WD, Bowen HK, Uhlmann DR. Introduction to ceramics: Wiley New York; 1960.

108. Chowdhry U, Sleight AW. Ceramic Substrates for Microelectronic Packaging. Annual Review of Materials Science. 1987 1987/08/01;17(1):323-40. 
109. Gangqiang W, Folk EC, Barlow F, Elshabini A. Fabrication of microvias for multilayer LTCC substrates. Electronics Packaging Manufacturing, IEEE Transactions on. 2006;29(1):3241.

110. Norell RA. Machine for filling via holes in an integrated circuit package with conductive ink. Google Patents; 1985.

111. Kuczynski GC. Self-diffusion in sintering of metallic particles. Trans Am Inst Min Eng,. 1949;185:169-78.

112. Coble RL. Sintering Crystalline Solids. I. Intermediate and Final State Diffusion Models. Journal of Applied Physics. 1961;32(5):787-92.

113. Young WS, Cutler IB. Initial Sintering with Constant Rates of Heating. Journal of the American Ceramic Society. 1970;53(12):659-63.

114. Fang T-T, Shiue J-T, Shiau F-S. On the evaluation of the activation energy of sintering. Materials Chemistry and Physics. 2003;80(1):108-13.

115. Wang J, Raj R. Estimate of the Activation Energies for Boundary Diffusion from RateControlled Sintering of Pure Alumina, and Alumina Doped with Zirconia or Titania. Journal of the American Ceramic Society. 1990;73(5):1172-5.

116. Shao W, Chen S, Li D, Cao H, Zhang Y, Zhang S. Apparent activation energy for densification of Al2O3 powder at constant heating-rate sintering. Bulletin of Materials Science. 2008;31(6):903-6.

117. Shao W, Chen S, Li D, Wan Y, Zhang Y, Zhang S, et al. Analysis of two grain size $\mathrm{Al} 2 \mathrm{O} 3$ sintering at low heating rate based on master sintering curves. Materials science and technology. 2008;24(6):734-8.

118. El-Aiat MM, KróGer FA. Determination of the Parameters of Native Disorder in $\alpha$ Al2O3. Journal of the American Ceramic Society. [10.1111/j.1151-2916.1982.tb10387.x]. 1982;65(3):162-6.

119. Bernard-Granger G, Guizard C, Addad A. Sintering of an ultra pure $\alpha$-alumina powder: I. Densification, grain growth and sintering path. Journal of Materials Science. 2007;42(15):631624.

120. Yeh T-S, Sacks MD. Effect of Particle Size Distribution on the Sintering of Alumina. Journal of the American Ceramic Society. 1988;71(12):C-484-C-7.

121. Ma J, Lim LC. Effect of particle size distribution on sintering of agglomerate-free submicron alumina powder compacts. Journal of the European Ceramic Society. 2002;22(13):2197-208. 
122. Lim LC, Wong PM, Jan M. Microstructural evolution during sintering of near-monosized agglomerate-free submicron alumina powder compacts. Acta Materialia. 2000;48(9):2263-75.

123. He Z, Ma J. Constitutive modeling of the densification and grain growth of fine-grained alumina ceramics. Materials Science and Engineering: A. 2003;361(1, Äi2):130-5.

124. Eskandari A, Aminzare M, Razavi hesabi Z, Aboutalebi SH, Sadrnezhaad SK. Effect of high energy ball milling on compressibility and sintering behavior of alumina nanoparticles. Ceramics International. 2012;38(4):2627-32.

125. Karagedov GR, Lyakhov NZ. Preparation and sintering of nanosized $\alpha$-Al2O3 powder. Nanostructured Materials. 1999;11(5):559-72.

126. Forrester J, Goodshaw H, Kisi E, Suaning G, Zobec J. Effect of mechanical milling on the sintering behaviour of alumina. J Aust Ceram Soc. 2008;44(1):47-52.

127. Vines RF, Semmelman JO, Lee PW, Fonvielle FP. Mechanisms Involved in Securing Dense, Vitrified Ceramics from Preshaped Partly Crystalline Bodies. Journal of the American Ceramic Society. 1958;41(8):304-9.

128. Coble RL. Sintering Alumina: Effect of Atmospheres. Journal of the American Ceramic Society. 1962;45(3):123-7.

129. Hassan SA. Kinetics of sintering of unsupported platinum catalyst in nitrogen, oxygen and hydrogen atmospheres. Journal of Applied Chemistry and Biotechnology. 1974;24(9):497503.

130. Glassl H, Kramer R, Hayek K. Electron microscopy of Pt/Al2O3 model catalysts : II. Sintering in atmospheres of H2, O2 and Ar. Journal of Catalysis. 1981;68(2):388-96.

131. Alcock CB, Hooper GW. Thermodynamics of the Gaseous Oxides of the Platinum-Group Metals. Proceedings of the Royal Society A: Mathematical, Physical and Engineering Sciences. 1960;254(1279):551-61.

132. Alcock CB. The Gaseous Oxides of the Platinum Metals. Platinum Metals Review. 1961;5(4):134-9.

133. Jehn H. High temperature behaviour of platinum group metals in oxidizing atmospheres. Journal of the Less Common Metals. 1984;100(Journal Article):321-39.

134. Lu G-Q, Sutterlin RC, Gupta TK. Effect of Mismatched Sintering Kinetics on Camber in a Low-Temperature Cofired Ceramic Package. Journal of the American Ceramic Society. [10.1111/j.1151-2916.1993.tb08311.x]. 1993;76(8):1907-14. 
135. Jean J-H, Chang C-R, Chen Z-C. Effect of Densification Mismatch on Camber Development during Cofiring of Nickel-Based Multilayer Ceramic Capacitors. Journal of the American Ceramic Society. [10.1111/j.1151-2916.1997.tb03132.x]. 1997;80(9):2401-6.

136. Jean J, Chang C. Camber development during cofiring Ag-based low-dielectric-constant ceramic package. Journal of Materials Research. 1997;12(10):2743-50.

137. Chang C-R, Jean J-H. Effects of Silver-Paste Formulation on Camber Development during the Cofiring of a Silver-Based, Low-Temperature-Cofired Ceramic Package. Journal of the American Ceramic Society. 1998;81(11):2805-14.

138. Chang J-C, Jean J-H. Camber Development During the Cofiring of Bi-Layer Glass-Based Dielectric Laminate. Journal of the American Ceramic Society. [10.1111/j.15512916.2005.00323.x]. 2005;88(5):1165-70.

139. Nagai Y, Hirabayashi T, Dohmae K, Takagi N, Minami T, Shinjoh H, et al. Sintering inhibition mechanism of platinum supported on ceria-based oxide and Pt-oxide,Äìsupport interaction. Journal of Catalysis. 2006;242(1):103-9.

140. Powell BR. Platinum sintering inhibition by yttrium oxide. Catalysis Letters 1992;15(Generic):189-97.

141. Dubrovinsky LS, Saxena SK. Emissivity measurements on some metals and oxides using multiwavelength spectral radiometry. High Temperatures - High Pressures. 1999;31(4):393-9.

142. Arblaster JW. Crystallographic properties of platinum. Platinum Metals Review. 1997;41:12-20.

143. Wimber R, Hills S, Wahl N, Tempero C. Kinetics of evaporation/oxidation of iridium. Metallurgical and Materials Transactions A 1977;8(Generic):193-9.

144. Owens WT, Rodriguez NM, Baker RTK. Carbon filament growth on platinum catalysts. The Journal of Physical Chemistry. 1992;96(12):5048-53.

145. Wu NL, Phillips J. Carbon deposition on platinum during ethylene oxidation. Journal of Catalysis. 1988;113(2):383-97.

146. Rhee SK. A study of the eutectics in the systems platinum-carbon and iridium-carbon: University of Cincinnati; 1966.

147. Grönbeck H, Tománek D, Kim SG, Rosén A. Does hydrogen pre-melt palladium clusters? Chemical Physics Letters. 1997;264(1, Äì):39-43.

148. Tanner LE, Okamoto H. The Pt-Si (Platinum-Silicon) system. Journal of Phase Equilibria. $1991 ; 12(5): 571-4$. 
149. McAlister A, Kahan D. The Al-Pt (Aluminum-Platinum) system. Journal of Phase Equilibria. 1986;7(1):47-51.

150. Shankland TJ, Waff HS. Partial Melting and Electrical Conductivity Anomalies in the Upper Mantle. J Geophys Res. 1977;82(33):5409-17.

151. Lee WH, Vanloon KR, Petrova V, Woodhouse JB, Loxton CM, Masel RI. The equilibrium shape and surface energy anisotropy of clean platinum. Journal of Catalysis. 1990;126(2):658-70.

152. Bréchignac C, Houdy P, Lahmani M. Nanomaterials and nanochemistry: Springer Verlag; 2007.

153. Welborn M, Tang W, Ryu J, Petkov V, Henkelman G. A combined density functional and x-ray diffraction study of Pt nanoparticle structure. The Journal of chemical physics. 2011;135(1):014503-5.

154. Corti CW. Sintering Aids in Powder Metallurgy. Platinum Metals Review. 1986;30(4):184-95.

155. Okuyama F. Crystalline tungsten grown by reducing vapor-deposited tungsten oxide. Journal of Crystal Growth. 1977;38(1):103-8.

156. Wu K, Jin Z. Thermodynamic assessment of the Al-Pt binary system. Journal of Phase Equilibria 2000;21:221-6.

157. Chauke HR, Minisini B, Drautz R, Nguyen-Manh D, Ngoepe PE, Pettifor DG. Theoretical investigation of the Pt3Al ground state. Intermetallics. 2010;18(4):417-21.

158. Foster LM, Long G, Hunter MS. Reactions Between Aluminum Oxide and Carbon The A12O3-A14C3 Phase Diagram. Journal of the American Ceramic Society. 1956;39(1):1-11.

159. Kim DE, Manga VR, Prins SN, Liu ZK. First-principles calculations and thermodynamic modeling of the Al-Pt binary system. Calphad. 2011;In Press, Uncorrected Proof.

160. McLean M, Hondros ED. A study of grain-boundary grooving at the platinum/alumina interface. Journal of Materials Science 1971;6(Generic):19-24. 
VITA

ALI KARBASI

ali.karbasi@fiu.edu

akarbasi@gmail.com

\section{EDUCATION:}

- Ph.D. student of Material Science and Engineering, Florida International University, Miami, FL, Spring 2009 - Summer 2012

- M.Sc. in Polymer and Coating Science, Amirkabir University of Technology (Tehran Polytechnic), Tehran, Iran, 2006

Total GPA: $17.69 / 20.0$

- B.Sc. in Polymer Coating Science and Engineering, Amirkabir University of Technology (Tehran Polytechnic), Tehran, Iran, 2004

Total GPA: $13.85 / 20.0$

\section{EXPERIENCE AND PROJECTS:}

- Developing a High Density Hermetic Feedthrough with High Temperature Cofired Pt/Al2O3, (PhD Thesis, Florida International University, Advanced Materials and Engineering Research Institute, 2009 - 2012)

- High Pressure Equation of State of the Transition Elements, (Florida International University, Center for Study of Matter at Extreme Conditions, 2009now)

- Improvement of Physical and Optical Properties of Automotive Clearcoat by the Use of Hybrid Organic- Inorganic System (M.Sc. Thesis, Amirkabir University of Technology and IranKhodro Co., 2006)

- Effect of nano-silica filler on the physical and mechanical properties of acrylicbased polyurethane clearcoats (As co. adviser in BSc. Thesis, Amirkabir University of Technology, 2006)

- Study of the Color Matching of Mass Colored Polypropylene (As research assistant, Amirkabir University of Technology and Research project Iran polymer and petrochemical research institute, Costumer oriented research and development, 2006)

- Study of the effect of nanoclay content on properties of a scratch resistant coating (Amirkabir University of Technology, 2005)

- Applications of Conductive Polymers Containing Carbon Nanotube (As a Seminar Lecture MS Course, 2005)

- Effect of Aluminium Flake Orientation on Coating Appearance (B.Sc. Thesis, Amirkabir University of Technology, 2004) 
- Studying the Effects of Primer Surfacer Properties on the Automotive Coating Appearance by DOE (Internship project, Irankhodro Car Manufacturing Company, 2003)

\section{PUBLICATIONS AND PRESENTATIONS:}

- High temperature interaction in Pt/A12O3 system. Part I: Reduced atmosphere environment, Manuscript under preparation

- High temperature interaction in Pt/Al2O3 system. Part II: Effect of carbon contamination, Manuscript under preparation

- Metastable phases in Pt-C system: High temperature synchrotron study, Manuscript under preparation.

- Materials Interaction in Cofired Platinum /Alumina High Density Feedthrough for Implantable Neurostimulator Applications, IMAPS-2011 conference, Long Beach, CA

- High P-T structure and P-V-T equation of state of hafnium, Journal of Applied Physics, Under Review

- The thermodynamics of several elements at high pressure, Calphad, Volume 35, Issue 1, March 2011, Pages 72-81

- Improving the performance of computer color matching procedures, Journal of Optical Society of America A, Vol. 25, Iss. 9, pp. 2251-2262 (2008)

- Computer Color Matching Procedures for Mass Colored Polypropylene, Polymer-Plastics Technology and Engineering, 47 (10), 1024 - 1031 (2008)

- Investigating the variations in properties of 2-pack polyurethane clear coat through separate incorporation of hydrophilic and hydrophobic nano silica, Progress in Organic Coatings, 59 (1), 81-87 (2007)

- Achievement of optimal aluminium flake orientation by means of special cubic experimental design, Progress in Organic Coatings, 57 (3), 175-182 (2006)

- A sol-gel route to obtain scratch resistance automotive nano coating, $2^{\text {nd }}$ Conference on Nanostructures (NS2008), 11-14 March, 2008, Kish Island, Iran

- Study of the effect of nanoclay content on some mechanical and optical properties of the resultant coatings, 2nd Coatings Science International 2006, June 26th - June 30th 2006, Noordwijk, Netherlands.

- Achievement of optimal aluminium flake orientation in metallic coatings by the special cubic experimental design, 2nd Coatings Science International 2006, June 26th - June 30th 2006, Noordwijk, Netherlands.

- Studying the Effects of Primer Surfacer Properties on the Automotive Coating Appearance by DOE, Color \& Appearance of Paint, 2 Day International Conference, Scandic Hotel, Copenhagen, Denmark, 10 - 11 May, 2006 